Die Belastung und Belastbarkeit von Schwimmtauchern unter besonderer Berücksichtigung der Konfiguration der Ausrüstung sowie des passiven und dynamischen Widerstands - dargestellt anhand von objektivierenden Untersuchungsmethoden

\author{
Dissertation \\ zur Erlangung des sozialwissenschaftlichen Doktorgrades der Sozialwissenschaftlichen \\ Fakultät der Universität Göttingen
}

\author{
vorgelegt \\ von \\ Thomas Droste \\ aus Essen an der Ruhr
}

Göttingen 2004 
1. Gutachter: Prof. Dr. Dr. André Niklas

2. Gutachter: Prof. Dr. Arnd Krüger

3. Gutachter: Prof. Dr. Gernot Badtke

Tag der mündlichen Prüfung: 13.1.2005 
Inhaltsverzeichnis

$1 \quad$ Einleitung ........................................................................................... 1-1

1.1 Über die Leistungsdiagnostik.......................................................... 1-4

1.2 Arbeitsphysiologie und Leistungsphysiologie ................................... 1-7

1.3 Die Tauchtauglichkeit aus arbeits- und sportphysiologischer Sicht..... 1-7

$1.4 \quad$ Literaturübersicht ................................................................................. 1-11

1.4.1 Zur Widerstandsmessung ........................................................ 1-11

1.4.2 Zur Leistungsdiagnostik im Wasser ............................................ 1-12

1.4.2.1 Indirekte Methoden................................................................ 1-13

1.4.2.2 Direkte Methoden ..................................................................... 1-15

1.4.3 Untersuchungen zum Energieverbrauch beim Tauchen........................ 1-17

1.5 Über die Biomechanik des Flossenschwimmen ................................. 1-22

1.5.1 Über das Flossenschwimmen ................................................ 1-22

1.5.2 Über die Biophysik des Flossenschwimmens .................................. 1-24

1.5.3 Über den passiven Widerstand unter Wasser ................................ 1-25

1.6 Über die arbeits- und sportmedizinische Spiroergometrie ................... 1-28

1.6.1 Ermittlung des Energieumsatzes ................................................ 1-29

1.6.1.1 Direkte Kalorimetrie ............................................................ 1-29

1.6.1.2 Indirekte Kalorimetrie ....................................................... 1-29

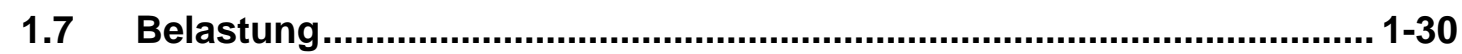

1.7.1 Belastung beim Tauchen ................................................. 1-30

1.8 Leistung .................................................................................... 1-32

1.9 Der dynamische Widerstand ........................................................... 1-34

1.10 Wirkungsgrad ............................................................................................ 1-35

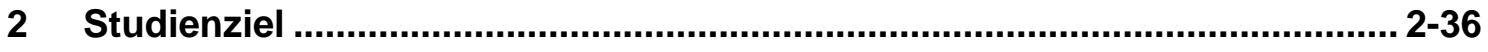

2.1 Studiendesign, Zielparameter............................................................ 2-36

2.2 Fragestellung und Hypothesen .................................................... 2-37

3 Material und Methoden ............................................................................... 3-38

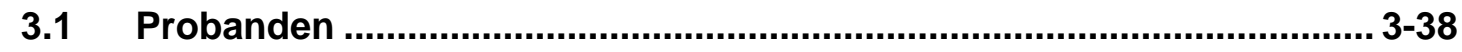

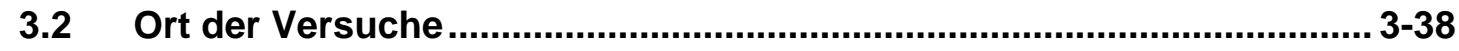

3.3 Methoden ............................................................................................... 38

3.3.1 Bestimmung der Strömungsverhältnisse im Kanal (Messreihe 1) .............

3.3.2 Und die Kalibrierung des Kraftaufnehmers.......................................3-38

unterschiedlichen Auftriebskörpern (Messreihe 2)........................... 3-39

3.3.3 Bestimmung der Strömungswiderstände der Probanden mit
verschiedenen Flossen (Messreihe 3) ........................................ 3-42

3.3.4 Spiroergometrische Untersuchung mit den Flossen aus der ...................... vorgenannten Messreihe (Messreihe 4) ......................................... 3-43

3.4 Statistische Methoden und Auswahl der Messwerte........................... 3-45

3.5 Probandensicherheit ........................................................................ 3-46

4 Entwicklung eines umgebungsdruckgesteuerten Ausatemventils............ 4-47

5 Ergebnisse ....................................................................................... 5-52 
5.1 Die realen Strömungsbedingungen im Strömungskanal (Messreihe 1)

und die Kalibrierung des Kraftaufnehmers

5.2 Die Strömungswiderstände $F_{D p}$ der Probanden mit

unterschiedlichen Auftriebskörpern (Messreihe 2)

5.2.1 Die Kraftwerte für jeden Auftriebskörper ....................................... 5-56

5.2.2 Gegenüberstellung aller Kraftwerte je Proband ............................... 5-59

5.2.3 Errechnete mechanische Leistung............................................ 5-60

5.3 Die Strömungswiderstände $\mathrm{F}_{\mathrm{Dp}}$ der Probanden mit verschiedenen Flossen (Messreihe 3).

5.4 Spiroergometrische Daten erhoben mit den Flossen aus der

vorgenannten Messreihe (Messreihe 4)............................................... 5-66

5.4.1 Anmerkungen zu Vorgehensweise und Versuchsabläufen ................ 5-66

5.4.2 Prüfung des Einschwingverhaltens............................................... 5-69

5.4.3 Respiratorischer Quotient ....................................................... 5-74

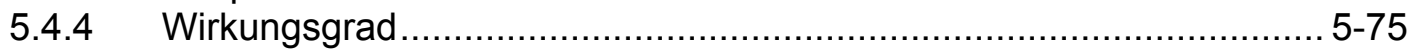

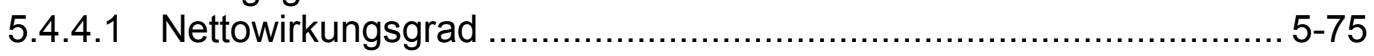

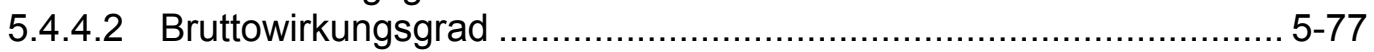

5.4.5 Mechanische Leistung ............................................................ 5-78

5.4.6 Dynamischer Widerstand .......................................................... 5-79

5.4.7 Vergleich der passiven und dynamischen Widerstandskraft .............. 5-82

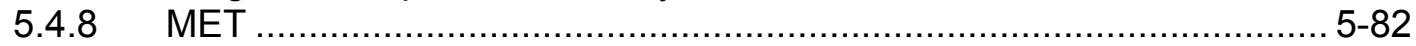

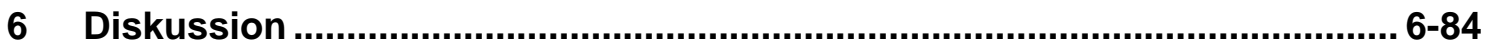

6.1 Diskussion der Methode.........................................................................6-84

6.1.1 Bestimmung der Strömungsverhältnisse im Kanal (Messreihe 1) .............. und die Kalibrierung des Kraftaufnehmers...................................... 6-84

6.1.2 Bestimmung der Kraftwerte für unterschiedliche .................................

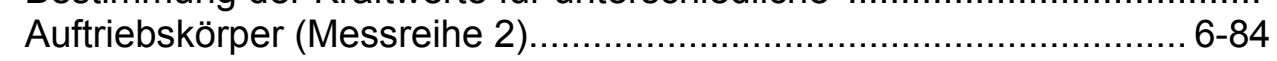

6.1.3 Bestimmung der Kraftwerte für unterschiedliche ....................................

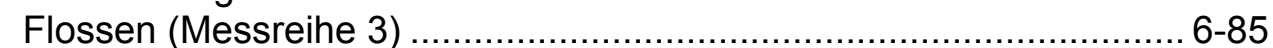

6.1.4 Die Spiroergometrie unter Wasser (Messreihe 4)........................... 6-85

6.2 Diskussion der Ergebnisse ................................................................... 6-88

6.2.1 Bestimmung der Strömungsverhältnisse im Kanal (Messreihe 1) ..............

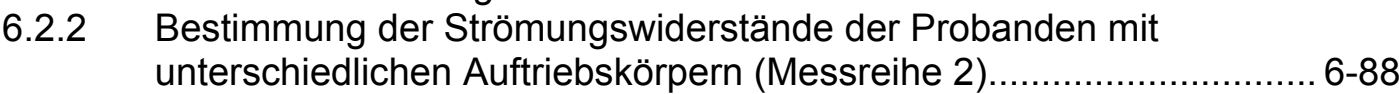

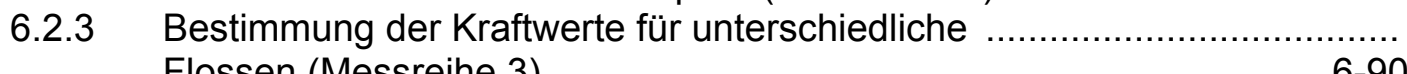

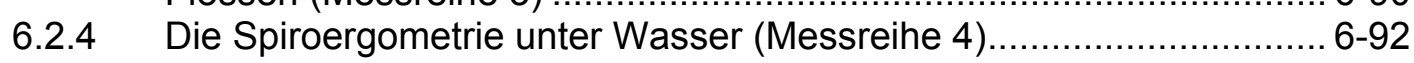

6.2.4.1 Respiratorischer Quotient....................................................... 6-92

6.2.4.2 Wirkungsgrad .................................................................... 63

6.2.4.3 Mechanische Leistung.......................................................... 6-94

6.2.4.4 Dynamischer Widerstand .................................................... 6-95

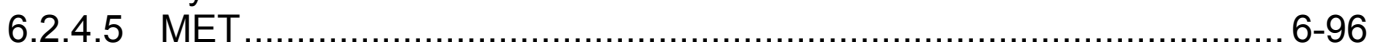

6.3 Überprüfung der Hypothesen ........................................................... 6-97

6.4 Zusammenfassung ......................................................................6. 6-98

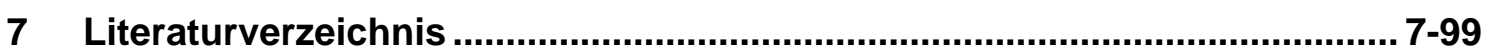


8.1 Dokumente, Messreihen und Daten

8.1.1 Umgebungsdruckgesteuerten Exspirationsventil.

8.1.2 Prüfprotokolle des Lungenautomaten mit umgebungsdruckgesteuerten Ausatemventil ....

8.1.3 Daten des Lungenautomaten Sherwood Brut.

8.1.4 Mittelwerte, Standardabweichung und Konfidenzintervall

8.1.5 Abstand zwischen Mittelwert und Median in den Schleppversuchen mit unterschiedlichen Auftriebskörpern (Messreihe 2).

8.1.6 Die Schiefe der Verteilung der Kraftwerte $F_{D p}$ von Proband A und $B$ mit unterschiedlichen Auftriebskörper (Messreihe 2)

8.1.7 Die Verteilung der größten und der kleinsten Schiefe je Proband (Messreihe 2)......

8.1.8 Abstand zwischen Mittelwert und Median in den Schleppversuchen mit unterschiedlichen Flossen (Messreihe 3)

8.1.9 Die Schiefe der Verteilung der Kraftwerte $F_{D p}$ von Proband A ... und $B$ mit unterschiedlichen Flossen (Messreihe 3 )

8.1.10 Die Verteilung der größten und der kleinsten Schiefe je Proband (Messreihe 3)

8.1.11 Messkurven aller Versuche (Messreihe 4) ................................... 8-122

8.1.12 Proband A. Messdaten (Messreihe 4) ........................................... 8-125

8.1.13 Proband B. Messdaten (Messreihe 4) .......................................... 8-128

8.1.14 Gegenüberstellung aller Messwerte mit den nach dem Einschwingverhalten relevanten Messwerten (Messreihe 4) ............ 8-131

8.2 Bilder.

Erklärung

Danksagung

Lebenslauf 


\section{Verzeichnis der Abbildungen}

Abbildung 1a: Testanordnung zur tätigkeitsspezifischen Spiroergometrie für Schwimmtaucher (definierte Leistung der oberen Extremitäten)

NIKLAS et al. $(1993,102)$.

Abbildung 1b: Testanordnung zur tätigkeitsspezifischen Spiroergometrie für Schwimmtaucher. „Ganzkörperarbeit“ - definierte Leistung am Seilzuggerät bei nicht fixiertem Körper NIKLAS et al. (1993,103). .. 1-18

Abbildung 2: „Bag in Box" System (Darstellung erstellt nach PENDERGAST et al. $(1996,574)$ )...

Abbildung 3: Kraulbeinschlag beim Flossenschwimmen

Abbildung 4: $\quad$ Flossenschwimmen mit der Monoflosse http://www.vdstflossenschwimmen.de/html/technik.html Zugriff am 11.6.2004

Abbildung 5: Widerstandswerte $F_{D p}$ in $\mathrm{kg}$ von geschleppten Schwimmern bei verschiedenen Geschwindigkeiten (geändert nach Clarys/Jiskoot),

UNGERECHTS $(1978,67)$.....

Abbildung 6: Schleppwiderstände von Schwimmern, modifiziert nach BINNER (1977, 467).

Abbildung 7: Der Schwimmkanal der Elbeschwimmhalle in Magdeburg. Eine ähnliche Konstruktion wurde von ÅSTRAND et al. (1972) benutzt. ALMELING (2004) Med-Habil. Göttingen (in Vorbereitung). Mit freundlicher Genehmigung des Autors.

Abbildung 8: Der Versuchsaufbau für Messreihe 2

Abbildung 9: Die Auftriebskörper aus Messreihe 2.

Abbildung 10: Versuchsaufbau für Messreihe 3 .

Die in Testreihe 3 und 4 untersuchten vier Flossenmodelle. Die Darstellung berücksichtigt annähernd das Größenverhältnis...... 3-43

Abbildung 12: Versuchsaufbau für Messreihe 3.

Abbildung 13: Umgebungsdruckgesteuertes Exspirationsventil.

Abbildung 14:

Fließdiagramm des umgebungsdruckgesteuerten Exspirationsventils. Strömungsrichtung, wie in Abb. 10 von rechts nach links. .......... 4-49

Abbildung 15: Messung der realen Strömungsverhältnisse in $470 \mathrm{~mm}$ Tiefe hinten mit $5 \mathrm{Mfl}$ bei eingestellten $0,3 \mathrm{~m} \cdot \mathrm{s}^{-1}$.

Abbildung 16: Messung der realen Strömungsverhältnisse in $80 \mathrm{~mm}$ Tiefe hinten mit $5 \mathrm{Mfl}$ bei eingestellten $1,2 \mathrm{~m} \cdot \mathrm{s}^{-1}$.....

Abbildung 17:

Abbildung 18: Mittelwerte der realen Geschwindigkeit an 5 Messflügeln differenziert nach $80 \mathrm{~mm}$ Tiefe, $470 \mathrm{~mm}$ Tiefe und dem Mittelwert beider Tiefen.

Mittelwerte der realen Geschwindigkeit an 3 Messflügeln differenziert nach $80 \mathrm{~mm}$ Tiefe, $470 \mathrm{~mm}$ Tiefe und dem

Mittelwert beider Tiefen.

Abbildung 19: Das Einschwingverhalten des Kraftaufnehmers.

Kalibrierung des Kraftaufnehmers.

Abbildung 21:

Kraftwerte $\mathrm{F}_{\mathrm{Dp}} /(\mathrm{N})$ für beide Probanden mit dem Wing Jacket. .. 5-57

Abbildung 22:

Abbildung 23:

Abbildung 24:

Kraftwerte $F_{D p} /(N)$ für beide Probanden mit der Kragenweste. ... 5-57

Kraftwerte $F_{D p} /(N)$ für beide Probanden mit dem HUB. ............. 5-58

Kraftwerte $\mathrm{F}_{\mathrm{Dp}} /(\mathrm{N})$ für beide Probanden mit dem Jacket............. 5-58

Abbildung 25:

Abbildung 26:

Widerstandswerte $F_{D p} /(N)$ aller Auftriebskörper für Proband A... 5-59

Abbildung 27:

Widerstandswerte $F_{D p} /(N)$ aller Auftriebskörper für Proband B... 5-60

Errechnete mechanische Leistung je Auftriebskörper für

Proband A.

Abbildung 28:

Errechnete mechanische Leistung je Auftriebskörper für 
Abbildung 29: $\quad$ Polynomische Darstellung der Kraftwerte $F_{D p} /(N)$ von

Proband $A$ mit vier unterschiedlichen Flossentypen.

Abbildung 30:

Polynomische Darstellung der Kraftwerte $F_{D p} /(N)$ von

Abbildung 31:

Proband $B$ mit vier unterschiedlichen Flossentypen. $5-67$

Abbildung 32:

Abbildung 33:

Abbildung 34:

Versuchsablauf Proband A mit Seemann Sub SF 2.

Versuchsablauf Proband B Mares Volo........................................... 5-68

Proband A Seemann Sub SF 2, alle Werte

$5-68$

der Belastungsstufen.

$5-69$

Abbildung 35:

Abbildung 36:

Abbildung 37:

Proband A Seemann Sub SF 2, Werte nach

$5-69$

der Einschwingphase.

$5-70$

Gesamtumsatz Proband A Mares Volo.

$5-71$

Abbildung 38:

Abbildung 39:

Abbildung 40:

Abbildung 41:

Abbildung 42:

Abbildung 43:

Abbildung 44:

Abbildung 45:

Abbildung 46:

Abbildung 47:

Gesamtumsatz Proband B Mares Volo.

Gesamtumsatz Proband A Mares Volo ohne Stufe $3(+5 \mathrm{~N}) \ldots \ldots . .5-71$
Gesamtumsatz Proband B Mares Volo ohne Stufe $3(+5 \mathrm{~N}) \ldots \ldots . .5-71$

Gesamtumsatz Proband A Sporasub Dessault. ......................... 5-72

Gesamtumsatz Proband B Sporasub Dessault. ........................ 5-72

Gesamtumsatz Proband A Seemann Sub SF 2 ....................... 5-72

Gesamtumsatz Proband B Seemann Sub SF 2 ....................... 5-73

Gesamtumsatz Proband B Monoflosse................................... 5-73

$R Q$ von Proband $B$ für vier Flossenmodelle. ............................. 5-74

$R Q$ von Proband $A$ für drei Flossenmodelle.............................. 5-75

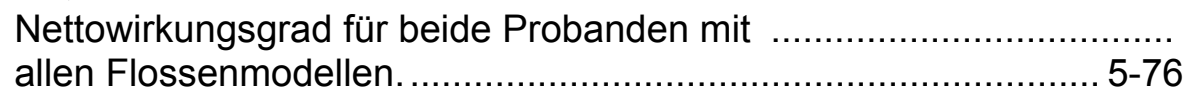

Abbildung 48:

Abbildung 49:

Abbildung 50:

Abbildung 51:

Abbildung 52:

Bruttowirkungsgrad für beide Probanden mit den Flossen

Mares Volo, Seemann Sub SF 2 und Sporasub Dessault........... 5-77

Bruttowirkungsgrad für Proband B mit der Monoflosse............... 5-78

mechanische Leistung Proband B mit vier Flossen..................... 5-78

mechanische Leistung Proband A mit drei Flossen..................... 5-79

Extrapolation des Arbeitsumsatzes Proband B

Sporasub Dessault.

Abbildung 53:

Extrapolation des Arbeitsumsatzes Proband A

Abbildung 54:

Sporasub Dessault.

Abbildung 55:

Seemann Sub SF 2.

$5-80$

Abbildung 56:

Extrapolation des Arbeitsumsatzes Proband A

Extrapolation des Arbeitsumsatzes Proband B Mares Volo. ........ 5-81

Abbildung 57:

Abbildung 58:

Abbildung 59:

Abbildung 60:

Extrapolation des Arbeitsumsatzes Proband B Monoflosse.

MET Proband A drei Flossen

$5-83$

MET Proband B vier Flossen.

$5-83$




\section{Verzeichnis der Tabellen}

Tabelle 1: Berücksichtigung von Leistungstests in Untersuchungsgrundlagen zur Tauchtauglichkeit.

Tabelle 2:

Charakteristika der in Messreihe 3 und 4 getragenen Flossen.

Tabelle 3:

Versuchsablauf Messreihe 4.

Tabelle 4:

Kenndaten des umgebungsdruckgesteuerten Exspirationsventils.

Neben den SI-Einheiten sind die Daten der Prüfprotokolle der Fa

Tabelle 5:

Schmidts und des Schreibens der Fa. Balzer übernommen worden.

Tabelle 6:

Reale Geschwindigkeiten im Strömungskanal...

Kraftwerte für Proband A.

Tabelle 7:

Kraftwerte für Proband $\mathrm{B}$.

Tabelle 8:

Bestimmtheitsmaß der Kraftkurven jedes Auftriebskörpers.

Tabelle 9:

Mittelwerte $P$ je Proband je Geschwindigkeitsstufe. Differenzwerte in

Prozent und Watt.

Tabelle 10:

Differenz zur durchschnittlichen errechneten mechanischen

Leistung in Watt bei Proband A.

Tabelle 11:

Differenz zur durchschnittlichen errechneten mechanischen

Tabelle 12: Leistung in Watt bei Proband B

Tabelle 13:

Kraftwerte mit Flossen für Proband $A$

Tabelle 14:

Kraftwerte mit Flossen für Proband $\mathrm{B}$.

Tabelle 15:

Bestimmtheitsmaß der Kraftkurven jeder Flosse.

Tabelle 16:

Tabelle 17: Anzahl der ausgewählten Messwerte in Messreihe 4.

Standardabweichung, Konfidenzintervall und Anzahl der in die Auswertung eingeflossenen Werte für beide Probanden. Auf die rot markierten Daten wird nachfolgend genauer Bezug genommen. .... 5-73

Tabelle 18: Gegenüberstellung von $F_{D p}$ und $F_{D d}$.

$F_{D p}$ mit Flossen für Proband $A$ im Vergleich mit den Ergebnissen von KARPOVICH et al. (1939) und HOLMÉR (1974).

Tabelle 19: $F_{D p}$ mit Flossen für Proband $B$ im Vergleich mit den Ergebnissen von KARPOVICH (1939) und HOLMÉR (1974).

Tabelle 20: $\dot{V} \mathrm{O}_{2}$ in verschiedenen Untersuchungen.

Tabelle 21:

Tabelle 22: Vergleich der Ergebnisse dieser Untersuchung mit den Ergebnissen von NIKLAS (2000) zur UW-Schwimmergometrie. Mit freundlicher Genehmigung des Autors. 
Verzeichnis der Abkürzungen

Maße, Einheiten

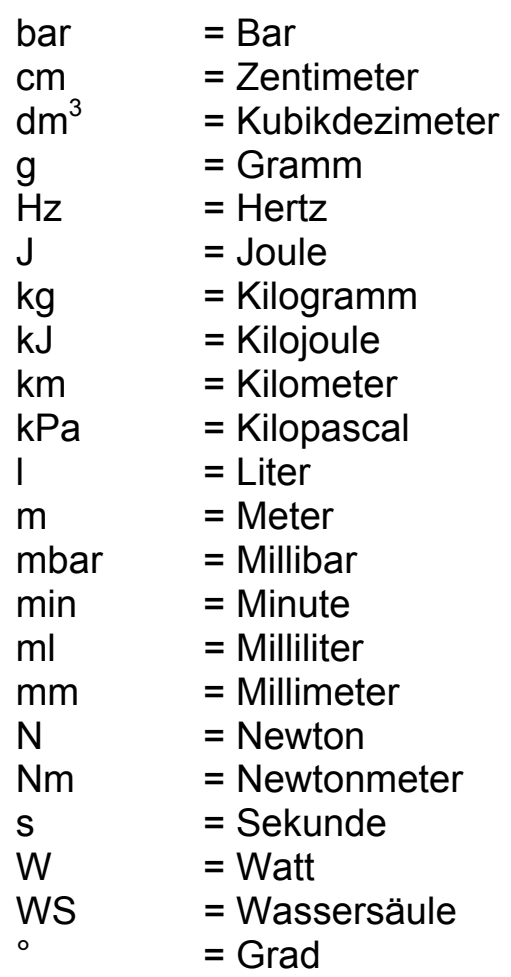

\section{Symbole}

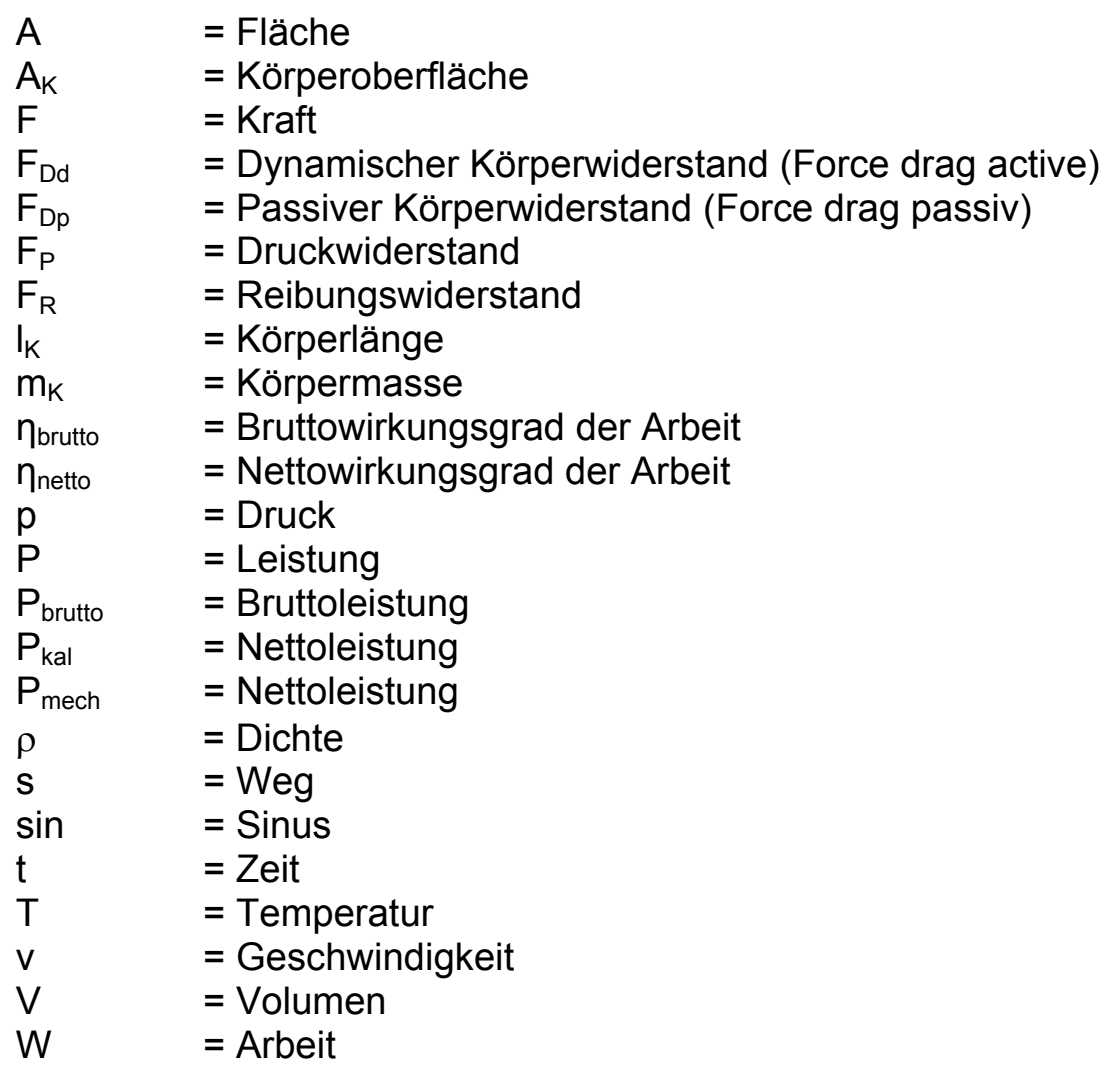




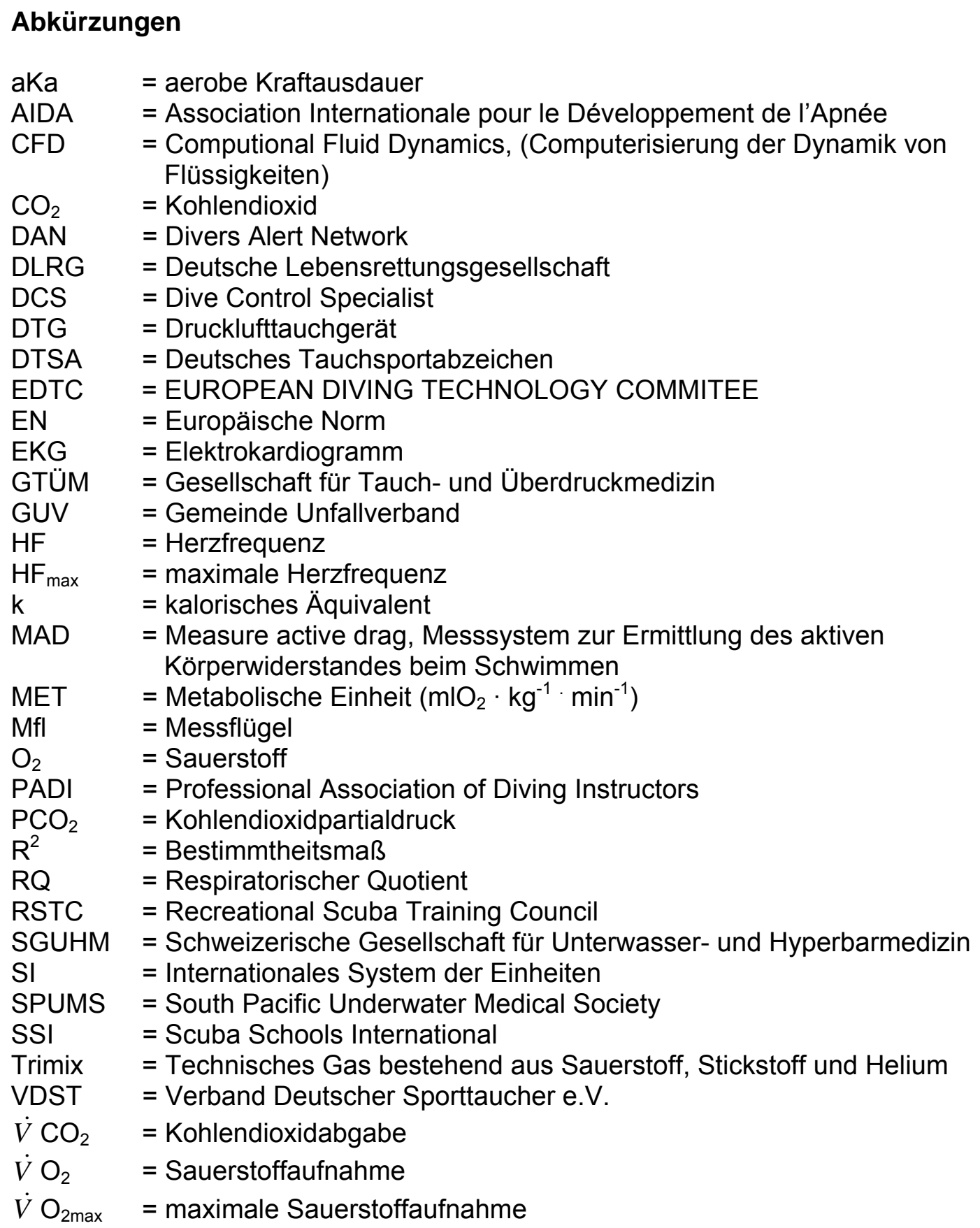


Das Tauchen hat sich in den letzten fünfzig Jahren von einer waghalsigen Unternehmung, der augenscheinlich nur einige "Lebensmüde“ nachgegangen sind, zu einer Freizeitbeschäftigung für Millionen von Menschen entwickelt. Es hat sich von einer eher sportlichen Ausprägung wie Harpunieren, Tieftauchen in Apnoe und dem Ausloten persönlicher physiologischer Grenzwerte zu einer Erholungssportart verändert, bei der das Erleben einer fremden Umgebung in völliger Entspannung das Hauptmotiv zu sein scheint. Um den Bedarf an Tauchsportartikeln, Tauchreisen, Tauchausbildung $\mathrm{zu}$ decken ist eine Tauchsportindustrie entstanden mit Tauchgeschäften, Spezialreiseveranstaltern, Produzenten und Tauchschulen. Im Tauchtourismus wurde 1996 ein Jahresumsatz von 1.942.000.000 Euro allein in Europa errechnet. Rückwirkend wurde für das gleiche Jahr in Europa ein Umsatz von ca. 270 Millionen Euro errechnet, basierend auf Ausrüstungsverkäufen und Ausbildung von Neukunden RSTC $\left(1997,6 f^{1}\right)$. Das Freizeittauchen stellt somit einen erheblichen Wirtschaftsfaktor mit steigender Tendenz dar.

Seit den Anfängen des Tauchsports in den fünfziger Jahren des vergangenen Jahrhunderts gibt es für die Gruppe von Tauchern, die das Tauchen zu ihrer Freizeitgestaltung betreiben, keine eindeutige begriffliche Zuordnung. Nennungen wie Sporttaucher, Freizeittauchen, Urlaubstaucher werden immer wieder teils voneinander abgrenzend aber auch übergreifend benutzt. Während Urlaubstaucher benutzt wird, um die Gruppe derjenigen, die dieses Hobby nur im Urlaub ausüben, zu kennzeichnen, wird Sporttaucher benutzt, um deutlich zu machen, dass Tauchen nicht nur im Urlaub, sondern gar als Sport ausgeübt wird. Andererseits wird der Begriff Sporttaucher sowohl abgrenzend zu reinen Freizeit- oder Urlaubstauchern verwendet, aber auch als gemeinsamer Oberbegriff für alle, die nicht erwerbsmäßig Tauchen SSI DCS Manual (ohne Datum, Anhang ohne Seitenangabe ${ }^{2}$ ), HOFFMANN (1995a, $9^{3}$ ), HOFFMANN (1995b, 1-14), LÜCHTENBERG (1988, 95), SCHULZ (1995, 106). Im Rahmen der Tauchtauglichkeit hat sich die Bezeichnung Sporttaucher als feststehender Begriff gebildet WENDLING $\left(2001,5,19^{7}\right)$, auch wenn er an anderer Stelle $\left(2001,9^{8}\right)$ von Freizeittauchen spricht. ALMELING $(2002 b, 4)$ stellt in Frage, „Ob das Tauchen den Zusatz „Sport“ zurecht trägt oder eher als Freizeitbetätigung gelten sollte“.

1 Die Zahlenangaben im Original sind in Deutsche Mark. Sie sind mit dem Umrechnungsfaktor 1,95583 in Euro umgerechnet und auf glatte Werte gerundet.

2 „• Freizeittauchen ist nicht gewerblich, militärisch oder technisches Tauchen“

3 "Der Tauchsport hat sich zu einer beliebten Freizeitsportart entwickelt."

4 "Tauchen gehört zu den Freizeitsportarten,..."

5 Lüchtenberg spricht an mehreren Stellen von der Sportart Tauchen, „aus der ....ein Breitensport für jedermann geworden (ist)“

${ }^{6}$ Schulz benutzt im Zusammenhang mit Tauchen die Begriffe „Sport“, „Sportart", „echte sportliche Betätigung",

7 „Das Sporttauchen.....(5) , „MEDIZINISCHE VORSORGEUNTERSUCHUNG VON SPORTTAUCHERN“ (19)

8 „Das Freizeittauchen mit Druckgasgerät..." 
Folgt man der begrifflichen Bedeutung von Beruf und Freizeit und damit dem Gegensatz, der zwischen beiden besteht, so erscheint diese Zuordnung sinnvoll und eindeutig. Berufstaucher üben mit dem Tauchen ihren Beruf aus und benutzen es zum Erwerb des Lebensunterhaltes. Taucher, die Tauchen in ihrer Freizeit ausüben, betreiben das Tauchen nicht erwerbsmäßig, wobei eine Grauzone festzustellen ist, auf die im Folgenden eingegangen wird.

Taucher in Bergungsunternehmen u.ä. und Tauchlehrer üben das Tauchen erwerbsmäßig aus. Zur Verwirrung trägt jedoch bei, dass noch lange nicht jeder, der seinen Lebensunterhalt durch das Tauchen bestreitet, den Beruf des Tauchers ausübt.

Die Bezeichnung Berufstaucher, welche die verkürzte Berufsbezeichnung für den anerkannten Abschluss "Geprüfter Taucher" BARTMANN (2002, VII - 2.1) ist, bezeichnet einen staatlich anerkannten Ausbildungsberuf. In der Berufstaucherei werden arbeitstechnische Aufgaben unter Wasser erledigt, sei es bei Arbeiten in Hafenanlagen, an Brücken, oder, wie in den letzten Jahren an der größten Unterwasserbaustelle Europas, dem Potsdamer Platz, etc..

Nach zwei Jahren Ausbildung in einem anerkannten Lehrbetrieb wird der „Jungtaucher" nach einer Prüfung bei der Industrie- und Handelskammer "Geprüfter Taucher". Darüber hinaus kann er die Prüfung zum Tauchermeister bei entsprechender Weiterbildung und Qualifikation ablegen.

Parallel dazu gibt es Bereiche, in denen nach den Regeln der Berufstaucherei getaucht wird, die aber zum großen Teil mit Teil-, bzw. Sonderqualifikationen zu bestimmten eingegrenzten Unterwasseraufträgen befähigen wie z.B. die Rettungstaucher der Deutschen Lebensrettungsgesellschaft und die Rettungstaucher der Wasserwacht des Deutschen Roten Kreuzes, die Taucher der Polizei und der Feuerwehr, die Forschungstaucher für Biologie, Archäologie etc., aber auch die Minentaucher der Marine.

Für den hauptberuflich, nebenberuflich oder ehrenamtlich arbeitenden Tauchlehrer gibt es weder ein eigenes Berufsbild noch fällt er unter das Berufsbild des Berufstauchers, obwohl er doch seinen Lebensunterhalt im Wesentlichen durch die Taucherei bestreitet, mit allen Nachteilen, die sich versicherungstechnisch ergeben wie der fehlenden Anerkennung von Berufskrankheiten.

Im Folgenden wird der Begriff Freizeittauchen für alle nicht erwerbsmäßigen taucherischen Tätigkeiten verwendet, während der Begriff Berufstauchen entsprechend der Bedeutung der Berufsbezeichnung benutzt wird. Auf die Grauzone, die sich um die Gruppe der Tauchlehrer bildet, wird jeweils gesondert eingegangen.

Weiterhin wird unterschieden in Helmtaucher, welche mit Taucherhelm und bleibeschwerter Taucherausrüstung mit einer direkten Gasversorgung von der Wasseroberfläche tauchen, und Schwimmtaucher, welche mit einer von ihnen selbst mitgeführten Gasversorgung tauchen. Schwimmtaucher bewegen sich zumeist mit Hilfe von Flossen vorwärts. 
Das Freizeittauchen ist mit der Entwicklung der Aqualunge durch Jacques Yves Cousteau während des 2. Weltkrieges entstanden SCHULZE (1995, 12f). Es ist immer schon durch die Übernahme von technischen Entwicklungen in die Sporttaucherei gekennzeichnet, die für Berufstaucher erarbeitet wurden. In der neuesten Zeit gibt es Entwicklungen, zusätzlich Tauchmethoden aus der Berufstaucherei für Freizeittaucher zu erschließen. So fließen im Spezialkurs Suchen \& Bergen Techniken der Rettungstaucher ein, im Spezialkurs Wracktauchen werden Techniken der Unterwasserarchäologen gelehrt und im Kurs Meeresbiologie werden Techniken der Forschungstaucher und Meeresbiologen angewendet. Im Bereich des Tieftauchens fällt die im Freizeittauchen gültige Empfehlung „40 m sind genug“ bzw. die Tiefengrenze von 40 Metern EHM $\left(1991,183^{9}\right)$, KERLL $\left(1994,64^{10}\right)$, SSI DCS Manual (ohne Datum, Anhang ohne Seitenangabe $\left.{ }^{11}\right)$, STIBBE $\left(1997,275,283^{12}\right)$, weil es durch Trimix- und Mischgas-Tauchen möglich wird, unter Eingrenzen der Gefahren, die beim Tauchen in diesen Tiefen mit Pressluft entstehen, größere Tiefen aufzusuchen. Diese aufwendige Technik war bisher nur den Berufstauchern vorbehalten. Weiter ist das Tauchen mit Sauerstoffkreislaufgeräten zu nennen, welche im Ursprung bei den Kampfschwimmern der Marinen der Welt verwendet werden. Dieses wird von einigen wenigen Herstellern und Ausbildungsorganisationen für den Freizeittaucher angeboten.

Auch fällt eine vormals eherne Grenze: War es vor Jahren noch völlig undenkbar, dass Jugendliche unter 14 Jahren eine Tauchprüfung ablegen, so wird heute diskutiert, ob mit 6 oder mit 8 Jahren mit der Tauchausbildung begonnen werden kann. Ob allerdings immer nur neues Wissens über die mögliche Eignung von Kindern für das Tauchen mit einer parallelen Anpassung der Tauchausrüstung an kindliche Bedürfnisse zu diesem Ergebnis kommen lässt oder ob vielmehr der Wunsch, neue Kundenschichten dem Tauchsport zu eröffnen die Triebfeder ist, ist nicht immer eindeutig zuzuordnen. Aussagen über die Belastungsanforderungen, auf die Kinder beim Tauchen reagieren müssen, werden erst seit kurzem bereitgestellt ALMELING et al. (2002, II- 7.3, 3).

Mit der demografischen Entwicklung wird die Zahl der Taucher, die bis ins hohe Alter tauchen wollen und dieses auch tun werden, steigen. War Jacques Yves Cousteau noch eine Ausnahme, die nachzuvollziehen war, da er von Jugend an tauchte, so seien stellvertretend für die prognostiziert zunehmende Zahl der älteren Taucher die Unternehmerin Beate Uhse und die Filmregisseurin Leni Riefenstahl genannt, welche beide im Rentenalter mit dem Tauchen begonnen haben. Hier ist die Tauchmedizin mit qualifizierten Aussagen gefordert und die Ausbildungsorganisationen sind gehalten, entsprechende Ausbildungskonzepte zu erarbeiten.

Vergleichbares gilt für den Bereich Behindertentauchen. Tauchen wird sowohl in der Therapie als auch in der Rehabilitation eingesetzt. Das Tauchen kann dabei für Behinderte mehrere positive Aspekte verbinden: Es ist gemeinsam mit

9 „Die Empfehlungen aller Tauchexperten sehen eine Tiefe von 30 bis 40 m für Sporttaucher, je nach Erfahrungsstand, als maximale Grenze an."

10 „2. Überschreite nie die Tiefengrenzen von $10 \mathrm{~m}$ mit Sauerstoffgerät und $40 \mathrm{~m}$ mit Preßluftgerät.“

11 "• Die maximale Tiefe beträgt $40 \mathrm{~m}$ “

12 "Tauchgänge über $40 \mathrm{~m}$ Tiefe werden nicht berücksichtigt." S.275

„Tauchgänge über $40 \mathrm{~m}$ werden nicht berücksichtigt.“ S.283 
Nichtbehinderten durchführbar, hat ein gegenüber dem Nichtbehinderten ähnliches Beanspruchungsprofil, entlastet den gesamten Stützapparat, ermöglicht neue Körpererfahrungen und bereitet einfach Spaß. In den vergangenen Jahren haben sich die Angebote für Behinderte, im Tauchen Fuß zu fassen, verbreitert. Mehrere Verbände konkurrieren mit Ausbildungsangeboten und Richtlinien um den behinderten Kunden. Gleichzeitig werden von der Tauchmedizin differenzierte Aussagen zur Tauchtauglichkeit erwartet HELLWIG $(1997,45)$.

Deutlich ist, dass Tauchen in Abhängigkeit zu den Voraussetzungen des Ausübenden und den von ihm verfolgten Absichten ein komplexes Gebilde ist, in dem mit völlig unterschiedlichen Zielsetzungen die Welt unter Wasser aufgesucht werden kann. Diese unterschiedlichen Zielsetzungen können zu unterschiedlichen Leistungsanforderungen führen. Aber sich ändernde Bedingungen wie Notsituationen, Strömungsveränderungen, Wellengang etc. können zu von den geplanten Leistungsanforderungen abweichenden Belastungsprofilen führen.

Über die Leistungsanforderungen beim Tauchen, vor allem in Abhängigkeit zur Konfiguration der Ausrüstung, liegen nur wenige Untersuchungen vor NIKLAS et al 1993, DRÄGER 1999, Witten 2000 und HOFFMANN 2000. So sind Ausbilder, welche sich u.a. mit Belastungssituationen für ihre Tauchschüler auseinander setzen müssen, vor allem auf Erfahrungswerte angewiesen ${ }^{13}$.

\section{1 Über die Leistungsdiagnostik}

Die Leistungsdiagnostik ist definiert als ein Verfahren zur Ermittlung des Entwicklungsstandes, der Leistungsfähigkeit und der Leistungsbereitschaft durch Erfassung, Bewertung und Beurteilung von Kenngrößen, Kennlinien oder Merkmalen des aktuellen Leistungsvollzuges SCHNABEL, THIEß (1993, 533f). Durch sie kann der Leistungsstand eines Sportlers oder Patienten etc. analysiert und mögliche Leistungsveränderungen dokumentiert werden. Die gebräuchlichsten standardisierten Testmethoden sind das Gehen oder Laufen auf dem Laufband oder die Belastung auf dem Fahrradergometer. Zunehmend werden auch Tests entwickelt und eingesetzt, in denen die Leistungsfähigkeit von Athleten unter möglichst sportartspezifischen Bedingungen untersucht werden BRAUER/GOTTSCHALK (1996, 113); BAUER $\left(1991^{14}\right)$ können.

\footnotetext{
${ }^{13}$ Im Rahmen einer Veranstaltung befragte der Autor 150 Tauchlehrer nach dem Strömungswiderstand von Auftriebskörper. Für eine Unterscheidung wurden Aspekte, wie liegt der Ausrüstungsgegenstand gut am Taucher an,

- $\quad$ ist der Ausrüstungsgegenstand strömungsgünstig konstruiert und - $\quad$ wie ist die Lage der Auftriebskörper

genannt. Die Tauchlehrer konnten bei der Beurteilung der Fragestellung nur auf ihre Erfahrung zurückgreifen.

${ }^{14}$ Bauer entwickelte eine Methode zur Leistungsdiagnostik mit Hilfe der Atemgasanalyse für Kanuten. Die Probanden führten dazu Douglas-Säcke auf dem Kanu mit, in denen die verbrauchte Luft aufgefangen wurde.
} 
Je nach Fragestellung und Zielsetzung können im Rahmen der Leistungsdiagnostik Daten zur Laktatkonzentration im Blut, der Herzschlagfrequenz, der Sauerstoffaufnahme oder der Ventilation bestimmt werden. In klinischen Situationen werden EKG und Blutdruck abgeleitet oder auch spezifische Messgrößen wie z.B. Blutzucker, Insulin, etc. ermittelt BRAUER/GOTTSCHALK $(1996,113)$.

Die erhobenen Daten dienen der Einschätzung der Leistungsfähigkeit des Patienten oder Probanden. Auf Grund der gewonnenen Daten können Entscheidungen betreffend einer Therapie oder eines folgenden Trainings getroffen werden.

Eine Einteilung in 5 Typen nimmt MARTIN (1980, 150-151) vor:

1. Sportmotorische Tests:

Sie sind eine Art Gebrauchstests, bei denen unter Standardbedingungen Daten zu Merkmalen des individuellen (Konditions-)Eigenschaftsniveaus sowie des technischen und taktischen Leistungsniveaus erhoben werden um den allgemeinen Leistungszustand zu beschreiben.

2. Biomechanische Leistungsdiagnostik:

In diesen Tests werden die biomechanischen Einflussgrößen bestimmt. Vor allem wird der Einfluss der leistungsdeterminierenden und technischen Faktoren abgeschätzt und ihr Bezug zur Gesamtleistung hergestellt.

3. Standardisierte Wettkampf- und Spielbeobachtung:

Für diese Testart sind verschiedene Untersuchungsfelder beschrieben: die unmittelbare Wettkampfvorbereitung, das Wettkampfniveau, die komplexe sportmotorische Leistung, die Entwicklungstendenzen der Sportart, das Wettkampfgerät, Gruppenprozesse und Gruppendynamik, Bewertungsmaßstäbe, Mannschaftsführung und -betreuung, Bewertungsmaßstäbe undtrends sowie der Sportlertyp.

4. Sportmedizinische und biochemische Funktionsprüfung und Untersuchungen:

Die Herzfrequenz (HF), die maximale Sauerstoffaufnahmefähigkeit $\left(\dot{V} \mathrm{O}_{2 \max }\right)$, das Herzvolumen, der Säure-Basen-Status sowie die Bestimmung des aeroben und anaeroben Schwellenwertes sind die wichtigsten Parameter dieser sportmedizinischen Leistungsdiagnostik.

5. Psychologische Testverfahren:

In psychologischen Testverfahren geht es darum, auf Grund der erfassten Informationen pädagogisch, betreuend, beratend und trainingsmethodisch steuernd in den Trainingsablauf einzugreifen. Dazu müssen diagnostische Informationen über den Sportler und 
über die Rahmenbedingungen der sportlichen Leistung gewonnen werden.

Die einzelnen Verfahren dienen dazu, das momentane Leistungsvermögen, auch in Teilbereichen, zu erfassen. Dazu ist es nötig, den kausalen Zusammenhang der leistungsbestimmenden Faktoren aufzuzeigen, um die sportliche Leistung über die Veränderung dieser Faktoren in Hinblick auf die Sollwerte zu verändern. Dazu muss der Einfluss dieser Faktoren abgeschätzt werden können, denn daraus ergibt sich der Einfluss auf die Gesamtleistung.

An die diagnostischen Leistungstestverfahren werden folgende Ansprüche erhoben (vgl. LETZELTER 1978, S.110):

- Objektivität

Die ermittelten Ergebnisse sind unabhängig vom Untersucher, der Durchführung der Untersuchung, der Auswertung und der Interpretation der Messwerte.

- Validität

Die zu messenden Parameter müssen hinreichend exakt bestimmt werden können.

- Reliabilität

Ein Test ist dann reliabel (zuverlässig), wenn die sportmotorischen Merkmale eindeutig und fehlerfrei beschrieben werden können. Mängel können von den Messgerätschaften, aber auch vom Sportler ausgehen, wenn sich die Bedingungen, das Befinden, die Motivation etc. ändern. Die Messungen müssen hinreichend genau sein und damit wiederholbar.

LETZELTER $(1978,112)$ nennt als Nebengütekriterium zusätzlich die Trennschärfe und die Ökonomie des Testverfahrens. Trennschärfe beschreibt, dass die Dauer von Tests mindestens so lang auszulegen ist, dass Leistungsfortschritte unter Berücksichtigung von Messfehlertoleranzen eindeutig festzustellen sind. Die Testökonomie bezieht sich auf den Einsatz von Geräten und den Zeitaufwand. Folge ist, dass aufwendige Testverfahren lediglich im Hochleistungssport anwendbar sind. Zielstellung ist demnach, dass mit einem Minimum an Aufwand ein Maximum an diagnostischer Genauigkeit erzielt wird.

Um die Leistung exakt bestimmen zu können, müssen physikalische Messverfahren angewendet werden, in denen die Größen: Kraft, Arbeit und Leistung zur Anwendung kommen. 


\subsection{Arbeitsphysiologie und Leistungsphysiologie}

Arbeitsphysiologie und Leistungsphysiologie sind Teil der physiologischen Wissenschaften. In beiden kommen Leistungstests zum Einsatz. Die Leistungsphysiologie beschäftigt sich mit allgemeinen physiologischen Abläufen unter dem Gesichtspunkt erhöhter oder an die Grenze der Leistungsfähigkeit gehender Belastungen. Im Breitensport wird dabei der Gesundheitsaspekt für alle Altersklassen berücksichtigt. Im Leistungssport ist vor allem die Erfolgskontrolle der Trainingsmethoden unter physiologischer Sicht von Interesse. Zunehmend gewinnt die Erforschung der Grenze sportlicher Leistung und die Warnung vor Schädigungen durch übertriebenes Training an Bedeutung.

Die Arbeitsphysiologie betrachtet die physiologischen Wirkungsabläufe unter dem Aspekt der beruflichen Arbeit. Sie beschäftigt sich mit dem gesunden arbeitenden Menschen. Sie ist Grundlagenforschung und angewandte Forschung zugleich, da das Zusammenspiel der Organe und Organsysteme unter Arbeit systematisch erforscht und diese Erkenntnisse anwendet, um die Arbeitswelt an die Bedürfnisse und Möglichkeiten des arbeitenden Menschen anzupassen (vgl. STEGEMANN 1991, 2).

\subsection{Die Tauchtauglichkeit aus arbeits- und sportphysiologischer Sicht}

Dass aus unterschiedlichsten Zielsetzungen im Rahmen des Tauchens auch verschiedenartige Belastungen und damit Leistungen für den Taucher resultieren, erscheint folgerichtig. Es ist ein Unterschied,

ob eine Gruppe von Freizeittauchern im Roten Meer bei einer Wassertemperatur von $26^{\circ}$ Celsius einen Tauchgang von 50 Minuten auf 15 Meter Wassertiefe unternimmt oder ob ein Tauchlehrer nach seinem dritten Tauchgang, bei dem er Gruppen geführt und ausgebildet hat, noch einmal abtauchen muss, um den $15 \mathrm{~kg}$ schweren Bootsanker zu lösen,

- $\quad$ ob eine Gruppe von Freizeittauchern im Mittelmeer einen Tauchgang auf 15 Meter Wassertiefe in einem offenen Höhlensystem durchführt oder eine Gruppe von Höhlentauchern einen Tauchgang zur Erforschung eines unterirdischen Sees unternimmt, wobei die Taucher vorher die Ausrüstung durch die niedrigen Stollen eines Bergwerks über 10 Stunden bis an den See getragen haben, oder

ob eine Gruppe von Sporttauchern an einem meereskundlichen Seminar an der Ostsee teilnimmt und den geplanten Tauchgang wegen schlechtem Wetter in eine ruhige Bucht verlegt und einige Kilometer weiter eine Gruppe von Feuerwehrtauchern die Unter-Wasser-Suche nach einem vermissten Segler aufnimmt.

Leistungsdiagnostische Tests kommen in der Taucherei vor allem bei der Tauglichkeitsuntersuchung zum Einsatz. Dabei sind diese Untersuchungen der 
Arbeitsmedizin zuzuordnen bzw. von arbeitsmedizinischen Untersuchungen abgeleitet. $\mathrm{Zu}$ unterscheiden ist zwischen der ärztlichen Tauchtauglichkeitsuntersuchung für Freizeittaucher und der für Berufstaucher.

Für Berufstaucher kommen zwei gesetzlich geregelte Verfahren zur Anwendung. Zum einen ist dieses das Verfahren für die Bundeswehrtaucher nach den Regeln der Taucher-, U-Bootfahrer und Kampfschwimmer-Verwendungsfähigkeit und zum anderen das Verfahren der Berufstaucher, Feuerwehrtaucher, Polizeitaucher, Taucher des Bundesgrenzschutzes, der Rettungstaucher von DLRG und Wasserwacht und der Forschungstaucher nach den Grundsätzen der Berufsgenossenschaft (G 31) „Arbeiten in Überdruck“, wobei Taucher nach Grundsatz 31.1 und Überdruckarbeiter nach Grundsatz 31.2 untersucht werden BARTMANN (2002, VII-6.7.3., 1-8). Diese Tauchtauglichkeit ist der Arbeitsphysiologie zuzuordnen. In ihr geht es darum, dem Untersuchten zu attestieren, ob er für einen überschaubaren Berichtszeitraum in der Lage ist, entsprechend der Belastungen, die durch die Ausführung von Unterwasserarbeiten an inn und seinen Organismus gestellt werden, Leistung zu erbringen.

Auch wenn die Namensverwandtschaft vermuten lässt, dass der Tauchsport der Sportphysiologie zuzuordnen ist, so ist hier jedoch zu differenzieren. Zielsetzung der Sportphysiologie für den Breitensport ist unter anderem, den Gesundheitsaspekt in den Vordergrund zu rücken. Es gilt den Bewegungsmangel frühzeitig zu bekämpfen und die Motivation für Life-time-Sportarten zu wecken, die energetisch anspruchsvoll, aber nicht zu anstrengend sind STEGEMANN (1991, 6). Diesem Anspruch wird die Tauchtauglichkeit für Freizeittaucher nicht gerecht. In ihr geht es der Namensbedeutung folgend darum, ob eine Person dazu in der Lage ist, den Tauchsport auszuüben und damit folgt sie eindeutig arbeitsmedizinischen Zielstellungen.

Im Hochleistungs-Tauchsport wie dem Flossenschwimmen, Streckentauchen, Orientierungstauchen und Unterwasserrugby sind Kontrollen der Trainingsmethoden wünschenswert und aus sportphysiologischer Sicht von Bedeutung. Hier können Daten gewonnen werden, die zuverlässig die Leistung des Sportlers wiedergeben. Dieser Bereich der Trainingssteuerung ist für die genannte Zielgruppe sinnvoll und hat keine Beziehung zur Arbeitsmedizin, sondern ist der Sportphysiologie zugehörig. Er spielt in der Tauchtauglichkeit für Freizeittaucher keine Rolle.

Für Freizeittaucher gibt es im Gegensatz zum Berufstauchen national keine gesetzliche Regelung zur Tauchtauglichkeitsuntersuchung. Sie werden national, aber auch international, nach völlig unterschiedlichen Richtlinien physiologisch untersucht. Diese reichen von einem Selbstauskunftsbogen, auf den hier beispielhaft von PADI (2001) Bezug genommen wird, bis zu der Tauchtauglichkeitsuntersuchung der Gesellschaft für Tauch- und Überdruckmedizin, die sich an den Grundsätzen für Berufstaucher anlehnen WENDLING et al. (2001, 19-24). PADI erscheint ein Selbstauskunftsbogen ausreichend, um die Eignung des Gefragten für den Tauchsport feststellen zu können und nur wenn der Gefragte in irgendeiner Auskunft gesundheitliche Probleme andeutet, wird eine medizinische Untersuchung zu diesem 
Problem erwartet. Das aber setzt voraus, dass dem so Gefragten ein Problem bereits bekannt ist. Von Kritikern wie EHM (1991, 335), ALMELING (2002, 8), wird diesem Ansatz entgegen gehalten, dass die ärztliche Tauchtauglichkeitsuntersuchung gerade auch unbekannte gesundheitliche Probleme, die für die Ausübung des Tauchsports von Relevanz sind, aufdecken soll und sie stellen fest, dass ein Selbstauskunftsbogen sicherlich nicht ausreichend ist. Die Organisationen, die mit einem Selbstauskunftsbogen arbeiten, negieren folgerichtig auch jede arbeitsphysiologische und sportphysiologische Relevanz. Es besteht, ihrem Ansatz folgend, grundsätzlich kein Bedarf, die Eignung von Menschen für das Freizeittauchen aus arbeitsphysiologischer oder leistungsphysiologischer Sicht zu untersuchen.

Abgrenzend dazu wird von der Gesellschaft für Tauch- und Überdruckmedizin (GTÜM) ein physiologischer Leistungstest empfohlen und ab dem 40. Lebensjahr erwartet.

Eine Grauzone besteht für die Gruppe der Tauchlehrer. Sie bewegen sich im Grenzbereich zwischen Freizeittauchen und Berufstauchen. Im Sinne einer Ausbildungsverordnung eines Berufsverbandes üben sie keinen Beruf aus. Folglich gelten für sie auch nur die Anforderungen der „Medizinische(n) Vorsorgeuntersuchung von Sporttauchern" WENDLING et al. $(2001,19)$. Sie müssten also im Falle der Zugehörigkeit zu Verbänden, die eine Selbstauskunftsbogen akzeptieren, möglicherweise überhaupt nicht untersucht werden. Nach den Richtlinien der GTÜM müssten sie sich zwar der Regeluntersuchung unterziehen, aber erst ab dem 40. Lebensalter leistungsphysiologisch untersucht werden. Demgegenüber kann ihre Tätigkeit durchaus Belastungen erreichen, die denen von Berufstauchern entsprechen. ALMELING $(2002 b, 6)$ merkt an, dass bei inhaltlich folgerichtiger und somit nach der Intention korrekter Auslegung des berufsgenossenschaftlichen Grundsatzes 31 angestellte Tauchlehrer entsprechend eben dieser Richtlinie zu untersuchen sind. Zur Verwirrung trägt bei, dass in den einführenden Bemerkungen zur arbeitsmedizinischen Vorsorgeuntersuchung zwar ausdrücklich Tauchlehrer mit einem Arbeitsaufenthalt im Ausland von mehr als drei Monaten als Zielgruppe genannt werden und in den Grundsätzen G35 auch berücksichtigt sind, die Tauchlehrer im Inland dagegen unberücksichtigt bleiben BARTMANN (2002, VII-6.7.4, 10). Es erscheint realitätsfern, dass ein Tauchlehrer mit einer gültigen Tauchtauglichkeit für Sporttaucher nach GTÜM oder einem ausgefüllten Selbstauskunftsbogen nach PADI oder anderen, die ähnlich verfahren, sich noch einmal vor einem Auslandsaufenthalt auf einer Tauchbasis nach Grundsatz G35 untersuchen lässt. Es ist zu vermuten, dass das Wissen über diese Richtlinie zudem in Tauchlehrerkreisen kaum verankert ist. Es besteht keinerlei leistungsbezogene berufsgenossenschaftliche Verknüpfung im Versicherungsfall, so dass diese Untersuchungsanforderung zwar präventiv begründet ist, jedoch in der Folge eines Schadensfalles für eine Regulierung nach berufsgenossenschaftlichen Grundsätzen keine Rolle spielt.

WENDLING et al. $(2001,193)$ stellen fest, dass für die Beurteilung der taucherischen Leistungsfähigkeit derzeit keine spezifischen Verfahren verfügbar sind und empfiehlt das Fahrradergometer oder das Laufbandergometer. 
Im Rahmen der Feststellung der Eignung zum Tauchen werden Leistungstests wie folgt berücksichtigt ${ }^{15}$ :

\begin{tabular}{|c|c|c|}
\hline Medizinischer Verband & Organisation & Empfohlener Leistungstest \\
\hline $\begin{array}{l}\text { Gesellschaft für Tauch- und } \\
\text { Überdruckmedizin GTÜM (1998) }\end{array}$ & & $\begin{array}{l}\text { Untersuchung durch Mediziner / Ergometrie mit } \\
\text { Ausbelastung, fakultativ, ab } 40 \text { Jahre vorgeschrieben }\end{array}$ \\
\hline $\begin{array}{lr}\text { Schweizerische Gesellschaft } & \text { für } \\
\text { Unterwasser- } & \text { und } \\
\text { Hyperbarmedizin SGUHM (2001) }\end{array}$ & & $\begin{array}{l}\text { Untersuchung durch Mediziner / Fahrradergometrie mit } \\
\dot{V} \mathrm{O}_{2 \max } \text { Bestimmung }\end{array}$ \\
\hline \multirow[t]{4}{*}{$\begin{array}{l}\text { South Pacific Underwater Medical } \\
\text { Society SPUMS }\end{array}$} & & Untersuchung durch Mediziner / kein Leistungstest \\
\hline & $\begin{array}{l}\text { Professional Association } \\
\text { of Diving Instructors PADI }\end{array}$ & $\begin{array}{l}\text { Selbstauskunft / Ärztliche Untersuchung nur bei } \\
\text { Problemen / kein Leistungstest }\end{array}$ \\
\hline & $\begin{array}{l}\text { Scuba Schools } \\
\text { International SSI }\end{array}$ & $\begin{array}{l}\text { Selbstauskunft / Ärztliche Untersuchung nur bei } \\
\text { Problemen / kein Leistungstest }\end{array}$ \\
\hline & $\begin{array}{l}\text { Verband Deutscher } \\
\text { Sporttaucher } \\
\text { e.V. VDST } \\
\text { und assoziierte Verbände }\end{array}$ & $\begin{array}{l}\text { Untersuchung durch Mediziner / Ergometrie mit } \\
\text { Ausbelastung, fakultativ, ab } 40 \text { Jahre vorgeschrieben, } \\
\text { entsprechend GTÜM }\end{array}$ \\
\hline Berufsgenossenschaft & & G 31 und G 35, Ergometrie erforderlich \\
\hline
\end{tabular}

Tabelle 1: $\quad$ Berücksichtigung von Leistungstests in Untersuchungsgrundlagen zur Tauchtauglichkeit.

Es ist zu vermuten, dass die Tauchverbände, die einen Selbstauskunftsbogen entwickelt haben und favorisieren, diese Bögen benutzen, da es dadurch für den potentiellen Taucher einfach wird, den Weg zum Tauchen einzuschlagen. Andererseits ist ein Tauchanfänger kaum in der Lage die möglichen Risiken einzuschätzen. Zielstellung ist hier wohl eher, auf einem schnellen Weg den ausbildenden Tauchlehrer von einer Haftung freizustellen ALMELING (2002b, 8). Die Bandbreite bei den Leistungstests ist eben in den unterschiedlichen Absichten begründet. Möglicherweise aber auch im Fehlen eines die Belastung und somit die beim Tauchen erbrachte Leistung darstellenden Verfahrens.

Die Forderung von STEGEMANN (1991, 260): „Im Idealfall müsste man also für jede einzelne Sportart einen eigenen Ergometertyp entwickeln," erhält hier eine besondere Bedeutung.

\footnotetext{
${ }^{15}$ In der Darstellung ist nur eine Auswahl von Verbänden berücksichtigt, da es in Deutschland bereits eine Vielzahl tauchsporttreibender Organisationen gibt, die sich jedoch entweder an den Vorgaben von PADI oder der GTÜM anlehnen.
} 
Die Tauchtauglichkeitsuntersuchung für Berufstaucher kann ausschließlich von Medizinern durchgeführt werden, welche eine entsprechende Prüfung nach den Richtlinien des Gemeinde-Unfallverbandes (GUV) abgelegt haben.

In Deutschland kann die Tauchtauglichkeitsuntersuchung für Freizeittaucher von jedem Arzt ausgeführt werden. Einen Facharzt für dieses Gebiet gibt es nicht. Die Gesellschaft für Tauch- und Überdruckmedizin bietet über Fachmediziner Taucherarztlehrgänge an, in denen das für diese Untersuchungen notwendige Fachwissen vermittelt wird. Zumeist schließt sich an den Kurs die Prüfung durch einen Beauftragten des GUV zur Befähigung für die G31 an.

\section{$1.4 \quad$ Literaturübersicht}

In der Literatur sind verschieden Untersuchungen zu finden, die sich mit der Messung von Widerständen und der Leistungsdiagnostik von Schwimmern und Tauchern beschäftigen. Dabei werden zum Teil unterschiedliche Fragestellungen verfolgt und ebenso unterschiedliche Methoden angewendet. Im Folgenden werden die Untersuchungen inhaltlich sortiert und chronologisch geordnet vorgestellt. Sollten Arbeiten Gesichtspunkte zu mehreren der im Folgenden genannten Aspekte beinhalten, so wird auf diese Arbeiten entsprechend mehrfach verwiesen.

\subsubsection{Zur Widerstandsmessung}

Da bei der Fortbewegung im und unter Wasser ein Körper gegen einen Widerstand bewegt wird, beschäftigen sich Untersuchungen mit dem passiven aber auch dem dynamischen Widerstand, der bei der Vorwärtsbewegung des Körpers im Wasser zu überwinden ist.

KARPOVICH et al. (1939) und HOLMÉR (1974) ermittelten den Zugwiderstand von Schwimmern durch passives Ziehen. Sie stellten einen passiven Widerstand von $\mathrm{F}_{\mathrm{Dp}}=$ $29 \cdot v^{2}$ fest. Dazu definierten sie die Bedingungen, in denen der Widerstand eines Körpers im Wasser untersucht wird. Es erschien innen naheliegend, durch passives Ziehen bei gegebener Geschwindigkeit den Zugwiderstand zu ermitteln. Ihr Ergebnis beschrieb das Verhältnis Widerstandskraft $F_{D p}$ und Geschwindigkeit v. Nach KARPOVICH et al. (1939) wuchs der passive Widerstand mit dem Quadrat der Geschwindigkeit.

Di PRAMPERO et al. (1974) untersuchten 10 gut trainierte Schwimmer mit der Methode der Zusatzkraftbeaufschlagung beim Kraulschwimmen an der Wasseroberfläche. Die Versuche fanden in einem runden Pool mit einem Durchmesser von 58,6 m statt. $\mathrm{O}_{2}$ und $\mathrm{CO}_{2}$. wurden gemessen. Bei konstanter Geschwindigkeit wurden die Versuchspersonen mit einem Zusatzgewicht von einigen $\mathrm{N}$ bis zu $15 \mathrm{~N}$ belastet. Über die Regression der $\dot{V} \mathrm{O}_{2}$-Geraden auf die Achse der Zusatzkraft 
ermittelten sie den dynamischen Widerstand $F_{D d}$. Dieser war um $30 \%$ höher als der passive Widerstand $F_{D p}$.

BINNER (1977) maß den Wasserwiderstand von Schwimmern bei unterschiedlichen Geschwindigkeiten und berechnete die passive mechanische Schwimmleistung in Watt. Es wurden Werte für $F_{D p}$ von $30 \mathrm{~N}$ bis $60 \mathrm{~N}$ bei $1,00 \mathrm{~m} \cdot \mathrm{s}^{-1}$ bis $1,45 \mathrm{~m} \cdot \mathrm{s}^{-1}$ festgestellt.

UNGERECHTS (1978) verglich Untersuchungsergebnisse verschiedener Autoren, die an passiv geschleppten Schwimmern gewonnen wurden. Er stellte fest, dass die Kurven der Widerstandswerte aus den Untersuchungen von Counsilman, Schramm und Karpovich parabolisch verliefen. Ausnahme war die Untersuchung von Kent-Atha, deren Verlauf linear ist.

HOLLANDER in TOUSSAINT (1994) stellte in Untersuchungen mit dem MAD-System einen deutlichen Zusammenhang zwischen passivem Wasserwiderstand und der Körperlage des Probanden fest.

HOLLANDER (1994) stellte für das Schwimmen fest, dass aktiver und passiver Wasserwiderstand stark divergierten.

MARKER (1999) stellte fest, dass in den meisten Fällen eine Berechnung des Auftriebs und des Widerstandes umströmter Körper nicht möglich ist und empfahl den Widerstand in experimentellen Untersuchungen zu messen.

JAHNS (2000) wies auf den gleichen Zusammenhang hin.

ZAMPARO et al. (2002) untersuchten mit der Methode der Zusatzkraftbeaufschlagung die Effektivität von einem Flossenmodell an der Wasseroberfläche. Es wurde ein linearer Zusammenhang zwischen der Zusatzkraft und der Sauerstoffaufnahme $\dot{V} \mathrm{O}_{2}$ festgestellt. Über die Regression der Arbeitssauerstoffaufnahme fanden sie einen aktiven Widerstand beim Flossenschwimmen an der Wasseroberfläche von 22,9 N $\pm 5,4$ bei $0,8 \mathrm{~m} \cdot \mathrm{s}^{-1}$.

\subsubsection{Zur Leistungsdiagnostik im Wasser}

Da es nur wenige Untersuchungen zur Leistungsdiagnostik für Taucher gibt, werden hier auch Methoden und Untersuchungen vorgestellt, die sich mit Schwimmern beschäftigen und das Themengebiet dieser Arbeit betreffen.

In den arbeitsmedizinischen Untersuchungen wie der G 31 nach den Richtlinien der Berufsgenossenschaft ist die Fahrradergometrie WELSLAU (1977), WENDLING et al. (2001) standardisierter Untersuchungsbestandteil. Für die Untersuchung der ärztlichen Tauchtauglichkeit sahen beide Autoren die gleiche Untersuchungsmethode vor.

COSTILL (1971) machte eine definierte Leistung an Land und im Wasser zum Gegenstand seiner Untersuchung. Die Probanden waren bis zum Kinn ins Wasser 
eingetaucht. In verschiedenen Positionen wurden unter Belastung per mechanischem Tretkurbelergometer zwar die gleiche maximale $\mathrm{O}_{2}$-Aufnahme wie an Land festgestellt, jedoch für eine definierte Leistung der 1,5-fache $\mathrm{O}_{2}$-Bedarf.

STEINBACH et al. (1985) zweifelten die Aussagekraft von Untersuchungen mit dem Fahrradergometer im Rahmen der Leistungsbestimmung für Schwimmerinnen und Schwimmer an.

Dieser Zusammenhang war bereits von HOLMÉR und ÅSTRAND (1972) in ihrer Untersuchung festgestellt worden. In einem Zwillingsversuch untersuchten sie eine leistungsschwimmende und eine sportlich nicht mehr aktive Schwester. Sie stellten erhebliche Unterschiede in der Sauerstoffaufnahme beim Schwimmen fest, bei gleicher Sauerstoffaufnahme bei der Fahrradergometrie und beim Laufen.

Ebenso verglich HOLMÉR (1972) die Herzfrequenz und maximale Sauerstoffaufnahme beim Schwimmen im Verhältnis zum Radfahren. Er stellte eine signifikant niedrigere Herzfrequenz und maximale Sauerstoffaufnahme beim Schwimmen im Vergleich zum Radfahren fest. Die Untersuchungen von HOLMÉR fanden alle an Schwimmern im Schwimmkanal statt.

Den gleichen Zusammenhang belegten die Studien von MAGEL et al. (1969), McARDLE et al. (1969), McARDLE et al. (1971) und MAGEL (1971), im Vergleich von Schwimmen und Laufen.

BAUMGARTL (1987) stellte fest, dass Tauchen für eine Leistungsmessung ungeeignet ist, da keine zyklischen Bewegungen ausgeführt werden, die mechanische Leistung nicht ohne weiteres messbar ist und die Gewinnung von Daten kariopulmonaler und metabolischer Art unter Wasser erschwert ist.

BRÄUER et al. (1994) verglichen Laktatwerte im Verhältnis zur Herzfrequenz bei Laufband -, Fahrradergometrie und beim Streckentauchen im Trockentauchanzug mit Presslufttauchgerät in ansteigendem Tempo. Sie fanden gute Übereinstimmungen zwischen Laufbandergometrie und Tauchbelastung und deutliche Unterschiede zwischen Fahrradergometrie und Tauchbelastung und schlossen daraus, dass speziell die Fahrradergometrie keine Prognose für die Bestimmung der Leistungsfähigkeit beim Tauchen erlaubt.

Zur Ableitung der $\mathrm{O}_{2}$-Aufnahme kommen indirekte und direkte Methoden zur Anwendung.

\subsubsection{Indirekte Methoden}

a) in Apnoe

Methoden in Apnoe wurden vor allem im Schwimmen eingesetzt und eignen sich auch für das Tauchen, jedoch nur für Untersuchungen im statischen und dynamischen 
Apnoetauchen. Sie verbieten sich für das Gerätetauchen auf Grund der Gefahr von Lungenüberdruckunfällen.

KARPOVICH et al. (1944) ließen Probanden über eine Strecke schwimmen und dabei die Luft anhalten. Die Ausatemluft wurde nach Belastungsende aufgefangen. Sie berechneten den Energieverbrauch über den $\mathrm{O}_{2}$-Verbrauch.

ADRIAN et al. (1966) führten eine ähnliche Untersuchung durch. Es fanden bei submaximaler Geschwindigkeit $\mathrm{O}_{2}$-Verbrauchsmessungen statt, bei denen die Luft in einem Douglas-Sack aufgefangen wurde. Der Douglas-Sack wurde während der Messung am Beckenrand neben dem Probanden hergetragen.

MONPETIT et al. (1981) stellten eine Methode vor, bei der ein Schwimmer nach einer Belastung von mindestens 5 Minuten beim letzten Einatemzug die Luft anhielt, die dann sofort nach dem Schwimmen in eine Maske ausgeatmet wurde. Durch Rückwärts-Extrapolation der $\mathrm{O}_{2}$-Erholungskurve sollte der $\mathrm{O}_{2}$-Verbrauch während des Schwimmens bestimmt werden.

RINGE (2004 Med-Diss Göttingen (in Vorbereitung), mit freundlicher Genehmigung der Verfasserin) führte in einer Studie Untersuchungen mit Schwimmtauchern in statischer und dynamischer Apnoe durch, wobei jeweils nach Belastungsende die Ausatemluft analysiert wurde. Ergebnisse liegen noch nicht vor.

b) mit Stichproben

KRASTEV et al. (1964) benutzten das Douglas-Haldane-Verfahren zur Bestimmung des Gasaustausches, indem sie in regelmäßigen Abständen Ballons mit Exspirationsluft zur Wasseroberfläche aufsteigen ließen. Die Sauerstoffaufnahme lag mit $1660 \mathrm{ml} \cdot \mathrm{min}^{-1}$ in der gleichen Größenordnung wie bei anderen Sportarten mit hoher Kreislaufbelastung. Sie konnten einen großen Einfluss der Atemtechnik auf die Sauerstoffausnutzung feststellen.

FOLEY et al. (1967) asservierten in einem Vakuumtank während des Tauchgangs von jeder Exspiration eine Probe. Bei Geschwindigkeiten von $1,5 \mathrm{~km} \cdot \mathrm{h}^{-1}$ bis zur „maximal möglichen Geschwindigkeit" maßen sie $\mathrm{O}_{2}$-Aufnahmen von 970 bis $2940 \mathrm{ml} \cdot \mathrm{min}^{-1}$ in Abhängigkeit zur Schwimmgeschwindigkeit.

RUSSELL et al. (1972) führten mit dieser Ausrüstung Tauchgänge mit vorgegebener Geschwindigkeit bis 20 Meter Tiefe durch. Die mit der Tiefe zunehmende $\mathrm{O}_{2}$-Aufnahme erklärten sie mit dem kältebedingt höheren Energiebedarf.

DWYER und PILMANIS (1978) verglichen Fahrradergometrie an Land mit Tauchergometrie in unterschiedlichen Tiefen bis 30 Meter bei unterschiedlichen Geschwindigkeiten. In Zeitabstände wurden vom Exspirationsgemisch Proben in 10 Metallzylindern asserviert. Sie nahmen irrtümlich gleiche Wirkungsgrade an und eichten das Ergometer folgerichtig entsprechend der Annahme, dass bei gleicher $\mathrm{O}_{2}$ - 
Aufnahme gleiche Leistung vorliege. Die Herzfrequenz war abhängig von der Belastung und nicht von der Tiefe.

\subsubsection{Direkte Methoden}

Direkte $\mathrm{O}_{2}$-Messungen wurden entweder an einer Longe, beim freien Schwimmen, im Kreisbecken oder im Schwimmkanal durchgeführt. Der Proband trägt dabei zumeist eine Maske oder ein Mundstück und eine Nasenklammer. Die Einatemluft wird über einen Schlauch zugeführt und über einen Ausatemschlauch wird die Exspirationsluft abgeleitet.

Beim Schwimmen ist der Proband in der Bewegung stark eingeschränkt oder Anteile der Bewegung entfallen komplett, wie die Kopfrotation beim Kraulschwimmen, damit in jeder Kopfhaltung geatmet werden kann. Die Strömungswiderstände erhöhen sich durch Maske und Schläuche und verfälschen somit das Ergebnis.

Beim Tauchen wird zumeist die Einatemluft aus dem Lungenautomaten geatmet. Bis auf die Schläuche, die zur Ableitung der Ausatemluft benötigt werden, entspricht die Ausrüstung der normalen Tauchausrüstung.

DONALD und DAVIDSON (1954) verglichen die $\mathrm{O}_{2}$-Aufnahme von Schwimmtauchern mit der von Helmtauchern bei verschiedenen nicht standardisierten Belastungen, um den Gasbedarf von Tauchern berechnen zu können. Sie fanden eine deutlich höhere Sauerstoffaufnahme $\left(\dot{V} \mathrm{O}_{2 \max } 3600 \mathrm{ml} \cdot \mathrm{min}^{-1}\right)$ bei den Schwimmtauchern im Vergleich zu derjenigen der Helmtaucher mit $2350 \mathrm{ml} \cdot \mathrm{min}^{-1}$.

GOFF et al. (1957) untersuchten den Gasaustausch beim Unterwasserschwimmen im Strömungskanal. Sie benutzen einen Schlauch, der vom Mundstück des Lungenautomaten die Ausatemluft zur Wasseroberfläche leitete. Sie fanden ineffiziente Wirkungsgrade für das Tauchen von 1,2 bis $5,6 \%$, variierend nach Schwimmlage, Technik und Geschwindigkeit.

McARDLE et al. (1971) verglichen $\mathrm{O}_{2}$-Verbrauch und Herzfrequenz beim freien Schwimmen und beim Laufen auf dem Laufband. Die maximale Herzfrequenz war beim Laufen höher als beim Schwimmen. Es wurde festgestellt, dass bei beiden Disziplinen Herzfrequenz und $\mathrm{O}_{2}$-Verbrauch linear voneinander abhängig sind.

DI PRAMPERO et al. (1974) und PENDERGAST et al. (1977) untersuchten den Energieverbrauch, indem sie die Probanden in einem Kreisbecken schwimmen ließen. Die $\mathrm{O}_{2}$-Aufnahme $\dot{V} \mathrm{O}_{2}$ wurde bei konstanter Geschwindigkeit gemessen und damit der Energieverbrauch bestimmt. Der Schwimmer war über ein Flaschenzugsystem mit einer Plattform verbunden und musste mit und ohne Zusatzgewichte schwimmen. Unberücksichtigt blieb, dass durch das Schwimmen im Kreis die Kräfte nicht ausschließlich in Vortriebsrichtung wirken.

PILMANIS et al. (1977) entwickelten ein Unterwasserergometer in Form eines Brettes, welches ein Taucher vor sich herschiebt. Die Leistung ergab sich aus der Kompression der Feder. 
DWYER (1983) schloss in einer weiteren Untersuchung mit dieser Ausrüstung, dass Herzfrequenz und Ventilation keine Aussage hinsichtlich der $\mathrm{O}_{2}$-Aufnahme $\dot{V} \mathrm{O}_{2}$ gestatten.

TOUSSAINT (1988) und TOUSSAINT et al. (1988) entwickelten das MAD-System, bei dem ihrer Meinung nach keine Leistung verloren geht, da der $\mathrm{O}_{2}$-Verbrauch die Energie ist, welche zur Überwindung des Wasserwiderstandes dient, wohingegen der $\mathrm{O}_{2}$-Verbrauch beim freien Schwimmen den gesamten Energieverbrauch darstellt.

NIKLAS, HOTTOWITZ, FUHRMANN und PETER (1988) entwickelten ein Verfahren zur Leistungsdiagnostik von Schwimmtauchern, bei dem sie über ein Feinmanometer den Umgebungsdruck an der 2. Stufe des Lungenautomaten aufnehmen wollten und diesen auf einen justierbaren Manostaten an der Wasseroberfläche leiten, welcher sich im Luftstrom der Ausatemluft befindet. Dadurch erschien es innen möglich den Strom der Ausatemluft, manuell auf Umgebungsdruck geregelt, abzuleiten. Mittels der Methode der Zusatzkraftbeaufschlagung wird die zu verrichtende Arbeit definiert.

Nach dem Prinzip der Zusatzkraftbeaufschlagung maßen NIKLAS und PETER (1993) nach ihrer Methode die Flossenschwimmleistung von Berufstauchern im Schwimmkanal. Dabei wurde ein Wirkungsgrad von $5 \%$ gegenüber $25 \%$ bei der Fahrradergometrie ermittelt.

PENDERGAST et al. (1996) fanden einen Zusammenhang zwischen der Abtriebskraft der Flossen und $\dot{V} \mathrm{O}_{2}$. Sie stellten fest, dass bei zunehmendem Abtrieb der Flossen $\dot{V} \mathrm{O}_{2}$ steigt. Bei niedrigen Geschwindigkeiten wurde die Abtriebskraft durch kombinierte Frequenz- und Amplitudenerhöhung kompensiert. In den hohen Geschwindigkeiten trat dieser Effekt nicht auf.

DRÄGER (1999) maß mit Hilfe von Sauerstoffkreislaufgeräten den Sauerstoffverbrauch von Schwimmtauchern bei verschiedenen Tauchgeschwindigkeiten. Dabei folgte die Messarmatur dem Taucher in einer Gondel. Beeinträchtigungen entstanden auch dadurch, dass die Ausrüstung nicht einer Standardtauchausrüstung entsprach. Die Verbindung zwischen Taucher und Gondel durch ein dünnes Kabel stellte keine nennenswerte Behinderung dar. Es bestätigten sich die Ergebnisse, welche PENDERGAST (1992) mit offenen Systemen gewonnen hatte.

WITTEN (2000) maß bei unterschiedlichen Belastungen während eines Tauchgangs den Blutdruck der Probanden. Bei gleicher körperlicher Belastung, basierend auf der gemessenen $\mathrm{O}_{2}$-Aufnahme an Land und im Wasser, fand er diastolisch niedrigere und systolisch höhere Blutdruckwerte. Die unter Wasser gemessene Herzfrequenz war bei gleicher Leistung höher, die maximale Herzfrequenz jedoch niedriger als an Land, da die maximal erreichbare Belastung an Land deutlicher über der im Wasser lag.

HOFFMANN (2000) untersuchte bei verschiedenen konstanten Geschwindigkeiten die Herzfrequenz. Mittels dieser konnten Rückschlüsse auf die Effizienz von Flossen gezogen werden. 


\subsubsection{Untersuchungen zum Energieverbrauch beim Tauchen}

Zum Teil sind die im Folgenden genannten Untersuchungen bereits unter oben genannten thematischen Aspekten aufgeführt. Aus inhaltlichen Gründen erscheint es jedoch sinnvoll, die energetischen Aspekte dieser und der im Folgenden neu aufgeführten Untersuchungen hier gesondert zu nennen.

DONALD et al. (1954) verglichen die $\mathrm{O}_{2}$-Aufnahme $\dot{V} \mathrm{O}_{2}$ von Tauchern, die mit Flossen ausgerüstet waren und solchen, die Bleischuhe trugen. Dabei hatten Taucher mit Bleischuhen eine geringere Sauerstoffaufnahme $\left(\dot{V} \mathrm{O}_{2 \max }=2350 \mathrm{ml} \cdot \mathrm{min}^{-1}\right)$ verglichen mit den Tauchern, die Flossen trugen, deren $\dot{V} \mathrm{O}_{2 \max }=3600 \mathrm{ml} \cdot \mathrm{min}^{-1}$ lag. Begründet wurde dieser Zusammenhang mit der relativ größeren Muskelmasse der Beine, die bei den Tauchern mit Bleischuhen passiv waren.

GOFF et al. (1957) untersuchten im Schwimmkanal den $\mathrm{CO}_{2}$-Gasaustausch an 5 untrainierten Tauchern bei verschiedenen Geschwindigkeiten. Dabei wurde die Ausatemluft mittels eines Schlauches vom Mundstück des Lungenautomaten zur Wasseroberfläche abgeleitet. Flossenschwimmen stellte sich als höchst uneffektiv dar. Der Wirkungsgrad lag bei 1,2 bis $5,6 \%$.

WELTMANN et al. (1969) untersuchten, ob Leistungstest an der Wasseroberfläche geeignet sind, um Aussagen über die Leistungsfähigkeit von Tauchern treffen zu können. Dazu mussten die Probanden unter Wasser und an Land mit dem Armergometer Belastungen bewältigen sowie Steinblöcke bewegen und ein „PipePuzzle" zusammensetzen. Die Belastung auf dem Fahrrad wurde ausschließlich an Land durchgeführt. Gemessen wurden die Herzfrequenz, das Atemminutenvolumen und die Respirationsrate. Sie kamen zu dem Schluss, dass sich Untersuchungen an der Wasseroberfläche dazu eignen, die Leistungsfähigkeit von Tauchern zu beurteilen. Insbesondere die Herzfrequenz gäbe darüber Aufschluss. Weitere Untersuchungen seien jedoch nötig.

RUSSELL et al. (1972) ließen Probanden bei unterschiedlichen Umgebungsdrücken (1, 2 und 3 bar) in Ruhe und bei Belastung in einem See untersuchen, um die Auswirkung von Druck und Kälte auf die Probanden bestimmen zu können. Die Belastungswerte (Schwimmgeschwindigkeit $30 \mathrm{~m} \cdot \mathrm{min}^{-1}$ ) wurden mit den Ruhewerten der jeweiligen Tiefe verglichen. In größerer Tiefe stiegen dabei die Ruhewerte und Belastungswerte von $\dot{V} \mathrm{O}_{2}, \dot{V} \mathrm{CO}_{2}$, und $\mathrm{O}_{2}$-Puls signifikant an, während die Atemfrequenz abnahm. Als Grund führten sie die erhöhte Atemarbeit in der Tiefe und die dort herrschende Kälte an.

PILMANIS et al. (1977) entwickelten ein Unterwasserergometer. Es handelte sich dabei um ein Brett, welches der Taucher vor sich herschiebt und gegen dessen Widerstand er arbeitet. Das Brett war auf Federn gelagert und die Leistung ergab sich aus dem Produkt der Schwimmgeschwindigkeit und dem Widerstand des Brettes (d.h. der Kompression der Federn). Die Arbeit des Schwimmers konnte mit dieser Methode nicht bestimmt werden. In 10 und 30 Metern Tiefe wurde mit vier unterschiedlichen Widerständen des Brettes gemessen. Bei einem Widerstand von 2,4 kg ergab sich in 
10 Metern Wassertiefe ein $\dot{V} \mathrm{O}_{2}$ von $3280 \mathrm{ml} \cdot \mathrm{min}^{-1} \mathrm{und}$ in 30 Metern Wassertiefe ein $\dot{V} \mathrm{O}_{2}$ von $3470 \mathrm{ml} \cdot \mathrm{min}^{-1}$.

BRÄUER (1992) und BRÄUER et al. (1994) benutzen die von SIMON et al. (1983) entwickelte Ergometrie im Wasser, bei der eine Lichtschrittanlage die Geschwindigkeit vorgibt, um kardiozirkulatorische und metabolische Beanspruchungen beim Tauchen bei unterschiedlichen Geschwindigkeiten untersuchen zu können. Die ermittelten Werte des Belastungsverfahrens wurden anhand der Laktatwerte und der Herzfrequenz mit Werten, die bei der Fahrrad- und Laufbandergometrie ermittelt wurden, verglichen. Es herrschte Übereinstimmung zwischen der Laufbandergometrie und der Tauchbelastung. In der Untersuchung wurde belegt, dass die Fahrradergometrie für taucherische Eignungstests nicht geeignet ist, sondern lediglich einen allgemeinen Orientierungsrahmen bietet. Weiter wurde festgestellt, dass ein linearer Zusammenhang besteht zwischen Körpergröße und Leistungsfähigkeit von Tauchern, d.h. je größer der Taucher, desto größer seine Leistungsfähigkeit.

NIKLAS et al. (1993) stellten ein Verfahren und eine Vorrichtung zur tätigkeitsspezifischen Leistungsdiagnostik für Schwimmtaucher vor. Der Proband musste einen Schwimmtest durchführen und gegen ein Seilzugergometer arbeiten. Der Schwimmtest gleicht dem Test, den NIKLAS et al. (1988) mit Schwimmern durchgeführt haben. Im Schwimmkanal mit einer Tiefe von 1,3 m taucht der Proband mit der kompletten Tauchausrüstung. Unter Zusatzkraftbeaufschlagung werden bei festgelegten Strömungsgeschwindigkeiten metabolische Messgrößen ermittelt und so die Leistung bestimmt. Ein Wirkungsgrad von 5\% wurde errechnet und die unter Wasser zu erbringende Leistung als Schwerstarbeit eingestuft.

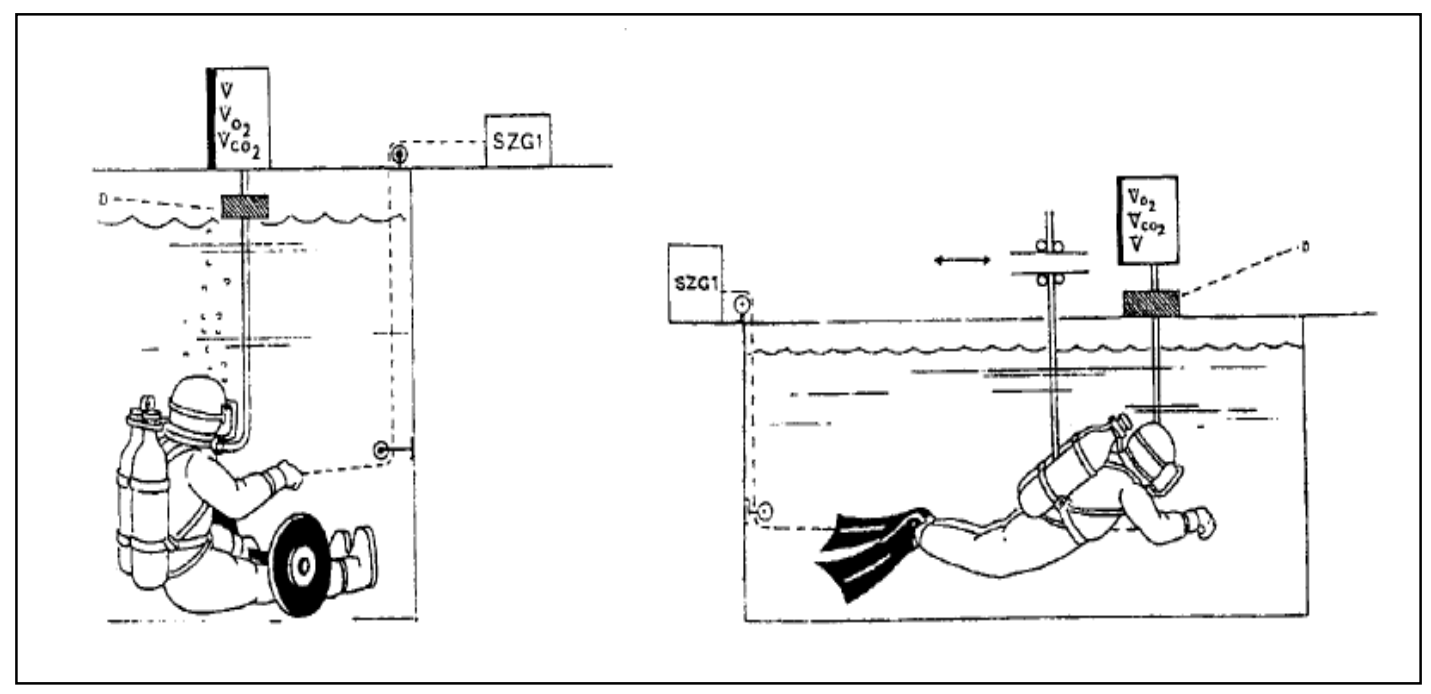

Abbildung 1a: Testanordnung zur tätigkeitsspezifischen Spiroergometrie für Schwimmtaucher (definierte Leistung der oberen Extremitäten) NIKLAS et al. $(1993,102)$.

Abbildung 1b: Testanordnung zur tätigkeitsspezifischen Spiroergometrie für Schwimmtaucher. "Ganzkörperarbeit" - definierte Leistung am Seilzuggerät bei nicht fixiertem Körper NIKLAS et al. $(1993,103)$. 
Mit dem Seilzuggerät sollte die Arbeit von Berufstauchern simuliert werden. Der Taucher sitzt dabei am Boden und zieht am Seilzugergometer.

Bei der dritten Testanordnung war der Taucher nicht fixiert, während er gegen das Seilzuggerät arbeitete. Durch die Annahme eines ähnlichen Wirkungsgrades konnten beide Tests über die Sauerstoffaufnahme verglichen werden. In der Folge mussten zwei von 19 getesteten Berufstauchern ihre berufliche Tätigkeit auf Grund von mangelnder physischer Leistungsfähigkeit aufgeben.

In ihrer Arbeit stellen PENDERGAST et al. (1996) ausgewählte Faktoren vor, die auf den Energieverbrauch beim Tauchen Einfluss haben. Die Probanden tauchten in einem 2,5 m tiefen Kreisbecken in der mittleren Tauchtiefe. Die Geschwindigkeit wurde durch eine Plattform, welche an einem Rotor befestigt war, vorgegeben. Dort war auch das "Bag in Box“ System angebracht. Da in dem „Bag in Box“ System der gleiche Umgebungsdruck herrschte wie in der Tauchtiefe von $1,25 \mathrm{~m}$, war eine Atemgasanalyse des unter Wasser ausgeatmeten Gasgemisches möglich.

Die Formel für Leistung und Energieverbrauch wurde folgendermaßen definiert:

$$
\begin{gathered}
\operatorname{Power}(\dot{W})=\text { BodyDrag }\left(F_{D p}\right) \cdot \operatorname{Velocity}(v) \\
\operatorname{EnergyCost}(\dot{E})=\dot{W} \cdot \text { Efficiency }(e)^{-1}
\end{gathered}
$$

Wobei sich die Geschwindigkeit wie folgt ergab:

$$
v=\text { kick rate } \cdot \text { distance per kick }
$$

Die Distanz pro Schlag wird von Art und Modell der Flosse bestimmt, wobei weitere Aspekte wie Beweglichkeit, Bewegungsmuster, Adaption an die Flosse und Umsetzung der optimalen Bewegung keine Rolle spielten.

Das Probandenkollektiv war leistungsheterogen zusammengesetzt. Der $\mathrm{O}_{2}$-Verbrauch stieg linear zur Schwimmgeschwindigkeit in allen Gruppen an. Die erfahrenen Taucher hatten den geringsten Sauerstoffverbrauch, während überraschenderweise die Gruppe der Berufstaucher den höchsten Sauerstoffverbrauch aufwies. Die Autoren vermuteten, dass diese während ihrer Unterwassertätigkeiten selten ausbelastet waren. Bestätigt wurde ein Ergebnis, das bereits bei Schwimmern festgestellt wurde, dass Frauen deutlich weniger Sauerstoff verbrauchen als Männer PENDERGAST et al. (1977). Die Ermittlung des Wasserwiderstandes geschah durch passives Ziehen. Dabei stellte sich heraus, dass der Wasserwiderstand an der Wasseroberfläche größer war als der Wasserwiderstand, welcher in 1,25 m Wassertiefe gemessen wurde. Weiterhin wurde in der Studie der Energieverbrauch mit unterschiedlichen Flossenmodellen untersucht und ein starker Einfluss von unterschiedlichen Flossenmodellen auf den Energieverbrauch festgestellt. 


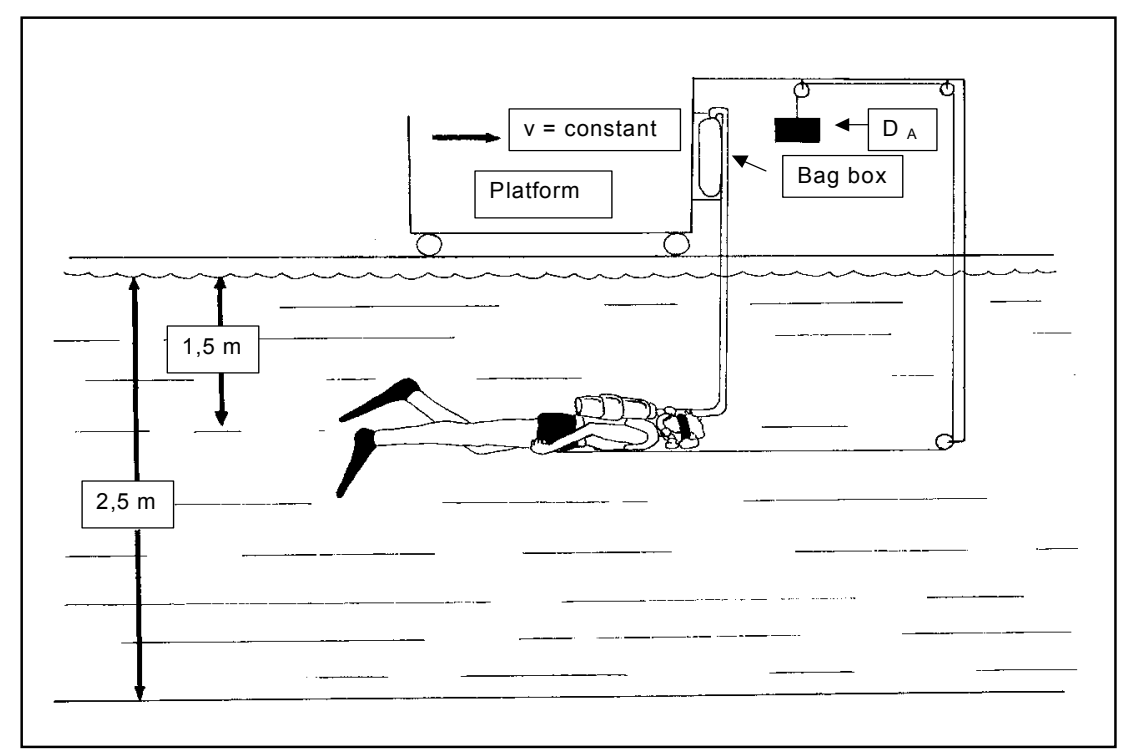

Abbildung 2: Bag in Box“ System (Darstellung erstellt nach PENDERGAST et al. $(1996,574))$.

Die Aussagekraft über den dynamischen Wasserwiderstand, ermittelt auf Grundlage der Messungen durch passives Ziehen (passiver Widerstand), wird von JAHNS $(2000,38)$ bezweifelt.

CHEN et al. (1996) stellte ein Unterwasser-Fahrradergometer vor. Damit sollten die physiologischen Antwortreaktionen des Körpers ermittelt werden. Die Ergebnisse wurden mit einer Fahrradergometrie an der Wasseroberfläche verglichen. Dabei wurden auch unterschiedliche Körperpositionen berücksichtigt. Eine eventuelle Mehrbelastung durch den Wasserwiderstand konnte nicht nachgewiesen werden. Jedoch wurde unter Wasser eine kürzer Belastungsdauer, eine geringere Maximalleistung, eine niedriger $\mathrm{HF}_{\max }$ und $\dot{V} \mathrm{O}_{2 \max }$ herausgefunden.

BUSLAPS et al. (1998) untersuchten Herzfrequenz, Atemminutenvolumen, Atemzugvolumen, $\quad \dot{V} \mathrm{O}_{2}, \quad \dot{V} \mathrm{CO}_{2}$ und die Blutlaktatkonzentration auf dem Fahrradergometer unter Normaldruck und unter Überdruck entsprechend $30 \mathrm{~m}$ Wassertiefe an 22 gesunden und trainierten Tauchern. In der Tiefe gab es zunehmende Einschränkungen bei der Ventilation mit gleichzeitig hochsignifikant vermindertem Atemminutenvolumen und reduzierter Atemfrequenz mit geringerem Atemzugvolumen. Bei höheren Belastungsstufen war eine gering erhöhte $\mathrm{O}_{2}$ Aufnahme und eine niedrigere $\mathrm{CO}_{2}$-Abgabe festzustellen. Unter Überdruck waren die Blutlaktatkonzentration und die HF hochsignifikant vermindert.

DRÄGER (1999) untersuchte die Sauerstoffaufnahme bei verschiedenen Schwimmgeschwindigkeiten mit Sauerstoffkreislaufgeräten unter Berücksichtigung der Herzfrequenz. Da es große individuelle Schwankungen gab, konnte keine Beziehung zwischen Herzfrequenz, Geschwindigkeit und Sauerstoffaufnahme festgestellt werden. Im Rahmen der Studie konnten die Probanden eine Geschwindigkeit entsprechend ihres Leistungsniveaus wählen. In 1,5 m Tiefe schwammen sie im Schwimmbad eine Strecke von $300 \mathrm{~m}$, die im Wesentlichen gerade war, an den Wendepunkten jedoch bogenförmig. Es standen 5 Geschwindigkeiten zur Auswahl. Das gemessene $\dot{V} \mathrm{O}_{2}$ lag 
bei den individuell geringsten Geschwindigkeiten im Mittel bei $2090 \mathrm{ml} \cdot \mathrm{min}^{-1} \mathrm{und}$ bei den höheren bei $2590 \mathrm{ml} \cdot \mathrm{min}^{-1}$.

NEUBAUER et al. (1999) untersuchten die Lungenfunktion von 15 Tauchern, während ihrer Ausbildung. Sie stellten bei den Probanden $(n=5)$, die Pressluft atmeten, keine relevante Verschlechterung nach den Tauchgängen fest. Die Probanden $(n=10)$, die Mischgas atmeten, wobei das Verhältnis von $\mathrm{N}_{2}$ und $\mathrm{O}_{2}$ je nach Tiefe variierte, wiesen nach den Tauchgängen eine reduzierte Lungenfunktion auf. Begründet wurde dieser Zusammenhang mit technischen Gegebenheiten der Tauchgeräte.

Aussagen zum Blutdruckverhalten beim Tauchen machte WITTEN (2000). Ziel der Untersuchung war es festzustellen, wann der Blutdruck bei Probanden kritische Werte annehmen konnte und ob dieses in der standardisierten Tauchtauglichkeitsuntersuchung erkannt worden wäre. Ein Vergleich der Fahrradspiroergometrie unter Wasser mit der über Wasser lieferte eine erhöhte Herzfrequenz bei Belastung unter Wasser, eine reduzierte $\dot{V} \mathrm{O}_{2 \max }$ und das frühere Erreichen der anaeroben Schwelle. Er stellte fest, dass eine zuverlässige Identifizierung unter Wasser auffälliger Probanden durch die Routineuntersuchung nicht gegeben war.

ZAMPARO et al. (2002) untersuchten an der Wasseroberfläche die Effektivität von einem Flossenmodell mit der Methode der Zusatzkraftbeaufschlagung. Die Probanden begannen die Versuche mit einer Zusatzkraft von $15 \mathrm{~N}$, die um $5 \mathrm{~N}$ vermindert wurde, bis sie mit $0 \mathrm{~N}$ schwammen. Es wurde ein linearer Zusammenhang zwischen der Zusatzkraft und der Sauerstoffaufnahme $\dot{V} \mathrm{O}_{2}$ festgestellt. Die Geschwindigkeit hatte keinen Einfluss auf diesen Zusammenhang.

In zwei Studien untersuchten PENDERGAST et al. (2003 a, b) mit einem männlichen und einem weiblichen Probandenkollektiv 7 handelsübliche und ein modifiziertes Flossenmodell. Die Tests wurden schwimmtauchend durchgeführt. Sie benutzen das "Bag in Box" System PENDERGAST et al. (1996) und maßen $\mathrm{VO}_{2}$ bei Geschwindigkeiten von 0,4 bis $0,9 \mathrm{~m} \cdot \mathrm{min}^{-1}$, sowie die maximale erzielte Geschwindigkeit, die maximale $\mathrm{O}_{2}$-Aufnahme und die maximale Kraft im steady state. Sie ermittelten $\mathrm{O}_{2}$-Aufnahmen $\dot{V} \mathrm{O}_{2}$ zwischen 950 und $2949 \mathrm{ml} \cdot \mathrm{min}^{-1}$ in Abhängigkeit zur Schwimmgeschwindigkeit. Sie fanden keinen Zusammenhang zwischen der Leistung einer Flosse und einer einzelnen Charakteristik der Flosse. Sie fanden keinen Zusammenhang zwischen größerer Härte einer Flosse und höherer Leistung. Zu weiche und $\mathrm{zu}$ harte Flossen zeigten sich als weniger geeignet für Männer. Die flexibleren Flossen waren für Frauen besser geeignet. 


\section{5 Über die Biomechanik des Flossenschwimmen}

\subsection{1 Über das Flossenschwimmen}

Beim Flossenschwimmen wird die Vortriebsleistung unter Wasser ausschließlich mit der Kraft der Beine, an denen Flossen getragen werden, bewältigt. An der Wasseroberfläche kann der Kraularmzug die Arbeit der Beine unterstützen. Beim Tauchen mit einem auf dem Rücken getragenen Tauchgerät ist die Ursache für die Unterlassung der Armbewegung in der eingeschränkten Armbeweglichkeit durch Neoprenanzug, Jacket und Flasche begründet. Mit der Flasche auf dem Rücken würde der Taucher durch die beim Kraularmzug forcierte Rollbewegung Gefahr laufen, auf den Rücken gedreht zu werden. Um dem entgegenzuwirken, wären ebenso starke Ausgleichsbewegungen notwendig. Zeitweise war im Prüfungskatalog des VERBANDES DEUTSCHER SPORTTAUCHER e.V.(1987) für das Deutsche Tauchsportabzeichen (DTSA) - Gold-Taucher III die Übung $100 \mathrm{~m}$ Streckenschwimmen mit ABC-Ausrüstung und 10 Liter Tauchflasche, jedoch ohne Benutzung des Lungenautomaten vorgesehen. Armbenutzung war dabei erlaubt. Zum Beispiel mussten männliche Erwachsene bis 40 Jahren die Strecke in 1:40,0 Minuten bewältigen, womit die Zeit 15 Sekunden unter der vergleichbaren Zeit des Deutschen Sportabzeichens für die $100 \mathrm{~m}$ Schwimmstrecke liegt. Aus der eigenen Praxis als Prüfungsberechtigter für beide Abzeichen weiß der Autor, dass beide Leistungen nicht vergleichbar sind. Die Leistungsanforderung des DTSA ist viel höher anzusiedeln als die des Sportabzeichens. Es ist zu vermuten, dass aus diesem Grund diese Übung nur kurze Zeit im Prüfungskatalog aufgeführt wurde. Die Ursache mag in einer vollkommenen Fehleinschätzung der zu erbringenden Leistung liegen. Zwar wurde bedacht, dass man sich mit Flossen schneller bewegen kann, jedoch wurde die ungünstigere Wasserlage durch die Tauchflasche, der dadurch geringere Anteil der Flossenbewegung für den Vortrieb und die ungünstige Rollbewegung bei Armbenutzung nicht bedacht, welche die insgesamt verbesserte Vortriebsleistung durch die Flossen mehr als wettmachen. Die Erfahrung zeigte, dass die besten Leistungen für das DTSA der einzelnen Probanden grundsätzlich schlechter ausfielen als die Leistungen für das Sportabzeichen.

Im taucherischen Leistungssport wird grundsätzlich auf die Armbewegung verzichtet. Beim Tauchen mit der Pressluft(Schiebe)flasche wird diese in Vorhalte vor dem Körper hergeschoben. Beim Streckentauchen in Apnoe werden die Arme in Vorhalte genommen, da in dieser Technik die schnellsten Zeiten erzielt werden. Im Flossenschwimmen kommen mit Duo- oder Stereoflossen und Monoflossen zwei Flossentypen zum Einsatz. Bei sogenannten Freiwasserflossen (Typ Duoflosse), die beim Gerätetauchen benutzt werden, trägt der Taucher zum Kälteschutz einen Füßling. Die Flosse wird mit einem Fersenband am Fuß gehalten.

Grundsätzlich ist die Bewegung mit Duoflossen oder Stereoflossen eine alternierende Bewegung mit beiden Beinen. Drehpunkt der Beinbewegung ist das Hüftgelenk. Die Bewegung kann in 4 Phasen unterteilt werden, von denen unten die Abfolge für das rechte Bein beschrieben wird: 


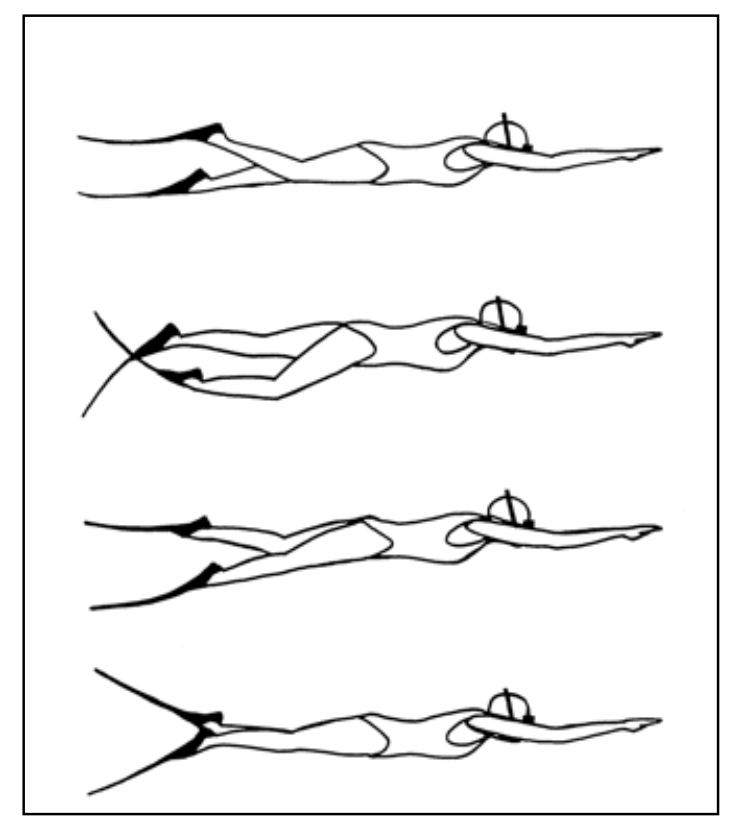

Abbildung 3: $\quad$ Kraulbeinschlag beim Flossenschwimmen HOFFMANN (1995, 4-3).

1. Phase: Nach der vollständigen Streckung des Beines beginnt der Abwärtsschlag aus der Hüfte (rechtes Bein), wobei das Kniegelenk passiv gebeugt wird.

2. Phase: Wenn das Kniegelenk den tiefsten Punkt erreicht, beginnt die aktive Streckung des Kniegelenkes. Zu Beginn dieser Phase ist das Flossenblatt maximal gebogen.

3. Phase: Das rechte Bein erreicht den tiefsten Punkt und ist gestreckt.

4. Phase: In der Ausholbewegung wird das rechte Bein locker gestreckt nach oben geführt (vgl. HOFFMANN 1995, 4-3).

Im Gegensatz zum Kraulschwimmen werden die Füße nicht nach innen gedreht AUSTE $(1978,36)$. MEYER (1972 ohne Seitenangabe) nennt eine maximale doppelte Schlagamplitude von $25-30 \mathrm{~cm}$ für das sportliche Flossenschwimmen (Abstand zwischen oberem und unterem Fuß).

Die obige Abbildung nimmt Bezug auf die im Breitensport beim Schnorcheln benutzte Ausrüstung und das entsprechende Bewegungsmuster. Im Gegensatz dazu wird beim sportlichen Flossenschwimmen mit längeren Flossen geschwommen, was zu einer größeren Amplitude führt. Auf die Taucherbrille wird zu Gunsten einer Schwimmbrille verzichtet und ein Mittelschnorchel wird eingesetzt, welcher vom Mund über die Nase in der Mitte des Kopfes zur Wasseroberfläche führt. Der Kopf wird zwischen die Arme genommen und diese neben, bzw. knapp oberhalb der Ohren maximal gestreckt nach vorne geführt.

Die Flossentechnik beim Schwimmen mit der Monoflosse ist eine einfache, symmetrische Bewegung. Die Hände werden gestreckt und zusammen in Verlängerung der Körperlängsachse gehalten. Der Kopf ist zwischen den Armen. Die Arme, der Oberkörper und die Beine führen eine Schwingungsbewegung aus. Die 
Amplitude dieser Bewegung ist in den Armen kaum merklich, steigert sich aber deutlich bis zum maximalen Umfang an den Füßen.

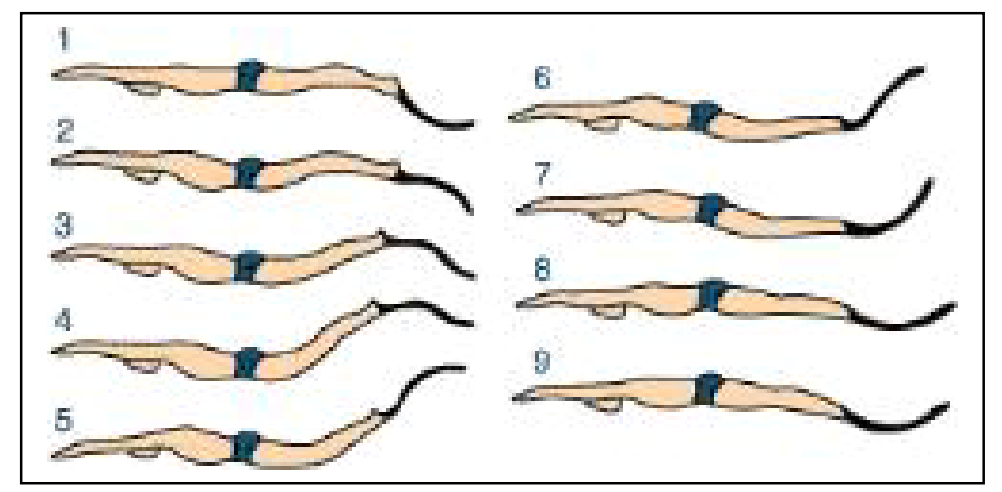

Abbildung 4: $\quad$ Flossenschwimmen mit der Monoflosse http://www.vdstflossenschwimmen.de/html/technik.html Zugriff am 11.6.2004.

Es ist zu bedenken, dass beim Tauchen mit Flossen die Optimierung der Bewegungsausführung in Kombination mit der Bewegungsadaption an das optimale Bewegungsmuster einer Flosse eine wesentliche Rolle spielt. Ebenso ist die Frage des Widerstandes durch Tauchausrüstungsgegenstände von großer Bedeutung.

\subsection{2 Über die Biophysik des Flossenschwimmens}

(vgl. UNGERECHTS et al. 2000)

UNGERECHTS et al. (2000) erklären Vortrieb im Wasser durch das Zusammenspiel dreier Antriebsimpulse, die mit drei Modellen erklärt werden. Dabei ist das 3. NewtonAxiom in jedem Fall beteiligt, auch wenn es sich um Vortrieb in einem Fluid handelt:

- $\quad$ Widerlagerbildung durch Nutzung eines (veränderbaren) Formwiderstandes

- Widerlagerbildung durch Nutzung des hydrodynamischen Auftriebs (Liftprinzip, Bernoulli)

- Widerlagerbildung durch Nutzung der Rotationsenergie in Verbindung mit einer Beschleunigungsaktion (Vortex-Prinzip)

Die Widerlagerbildung durch Nutzung eines, möglicherweise veränderbaren, Formwiderstandes findet bei der Paddelbewegung von Schwimmvögeln wie z.B. bei Enten ihre Entsprechung in der Natur. Hierbei wird ein Körper (Entenflosse) entgegen der Vortriebsrichtung bewegt und damit Wasser nach hinten beschleunigt. Die Flosse wird in der Gegenbewegung in ihrer Fläche verkleinert. Die Dynamik der Bewegung ist dem Bewegungsziel Vortrieb angepasst, so dass die Vortriebsbilanz insgesamt positiv ist. Anwendung findet dieses Prinzip in der Technik im Vortriebsmodell des 
Schaufelraddampfers und im Schwimmen beim zur Vortriebsrichtung senkrechten Einsatz der Hand, welche nach hinten bewegt wird.

Der hydrodynamische Auftrieb oder das Liftprinzip nutzt den Sog über und den Druck unter einem umströmten Körper, um Vortrieb zu erzeugen. Resultierend ist, dass an einem quer zur Vortriebsrichtung bewegten Körper Vortrieb entstehen kann. Das Prinzip findet bei der Schiffsschraube und der Flugzeugtragfläche in der Technik Anwendung. Beim Flossenschwimmen entstehen die gewünschten Sog- und Druckverhältnisse an der Flosse, welche durch das Wasser bewegt wird.

Das Vortex-Prinzip basiert auf der Entstehung rotierender Wassermassen durch die Flossenbewegung, von denen ein Abdruck möglich ist. Die entstehenden Wirbel sind dabei stationär und standortstabil. Sie bilden sich bei Körpern mit zyklischer Formveränderung (Delfin), bei dem hinter dem Körper im Nachlauf des Wassers eine Einrollbewegung der Strömung zu beobachten ist. Dabei ist festzustellen, dass die höchste Beschleunigung im Umkehrpunkt der Bewegung realisiert wird. Die Impulsübertragung entsteht durch das fast vollständige Abstoppen der Rotation des Wirbelballens, wodurch eine Impulsübertragung des Drehimpulses auf den Körper möglich wird. In der Biologie ist die Umsetzung des Vortex-Prinzips insbesondere beim Schwimmen der Wale und Delfine zu beobachten. Das Prinzip von stabilen, rotierenden Massen findet in der Technik Anwendung z.B. beim Kreiselkompass. Beim Flossenschwimmen ist der Effekt speziell beim Schwimmen mit der Monoflosse zu beobachten. Anfänger berichten in der Ausbildung immer wieder von „unerklärlichen“ Vortriebsspitzen oder einem „Turboeffekt“, welcher plötzlich eingesetzt habe, für den einzig und allein das Vortex-Prinzip verantwortlich ist.

\subsection{3 Über den passiven Widerstand unter Wasser}

Die Widerstandskraft $F_{D p}$ ist definiert als

$$
F_{D p}=c \cdot \frac{\rho}{2} \cdot v^{2} \cdot A
$$

Wobei $\mathrm{F}$ die Widerstandskraft in Newton, $\mathrm{c}_{\mathrm{w}}$ der Widerstandbeiwert, $\rho$ die Dichte des Mediums, v die Strömungsgeschwindigkeit und A die Stirnfläche des Körpers ist.

Während die Definition der Dichte des Mediums und die Geschwindigkeit nur geringe Probleme verursachen, ist die Bestimmung des c-Wertes und der Stirnfläche nur mit sehr großem Aufwand möglich. Die exaktesten Ergebnisse würde die Auswertung aller während der Strömungsexposition relevanten Daten ergeben. In der Schiffstechnik wird mit dem Verfahren CFD= Computional Fluid Dynamics die Simulation von turbulenten Strömungen mit gekoppelter Wärme-, Impuls- und Stoffübertragung ein entsprechendes Messverfahren angewandt. Die dazu nötigen Berechnungen dauern auf Hochleistungsrechner zwischen Stunden und Monaten, je nach Genauigkeitsanforderungen (vgl. HASSEL/LEDER 2003, 1).

Eine weitere Möglichkeit ist, den Widerstand zu berechnen. Dabei ergibt sich die Widerstandskraft $F_{D p}$ aus der Addition des aus dem Druck senkrecht zur 
Körperoberfläche gebildeten Druckwiderstands $F_{P}$ und des durch Schubspannung an der Grenzschicht auftretenden Reibungswiderstands $F_{R}$, die sich beide durch Integration über die Körperoberfläche berechnen lassen.

$$
\begin{aligned}
& F_{P}=\int \rho \cdot \sin \varphi \cdot A_{K} \\
& F_{R}=\int \rho \cdot \sin \tau \cdot A_{K}
\end{aligned}
$$

Die Körperoberfläche lässt sich nach der Formel von DU BOIS/DU BOIS(1916, 386) berechnen:

$$
A_{K}=m_{K}^{0,425} \cdot I_{K}^{0,725} \cdot 71,84 \cdot 10^{-4}
$$

Wobei $A_{k}$ die Körperoberfläche in $\mathrm{cm}^{2}, \mathrm{~m}_{K}$ die Körpermasse in $\mathrm{kg}$ und $\mathrm{I}_{K}$ die Körperlänge in $\mathrm{cm}$ ist.

Der menschliche Körper ist aber, da er hinreichend flexibel ist, in einer Strömung permanenten Anpassungsprozessen an die Strömungsverhältnisse unterworfen, so dass um den Körper herum sich verändernde Strömungssituationen entstehen und die o.a. Berechnung der komplexen Situation im Wasser für den menschlichen Körper nicht gerecht wird.

Der passive Widerstand $F_{D p}$ lässt sich auch mit der oben genannten Formel

$$
F_{D p}=c \cdot \frac{\rho}{2} \cdot v^{2} \cdot A
$$

bestimmen, wobei c der Widerstandsbeiwert, welcher abhängig von der Reynoldszahl ist und A die Stirnfläche, d.h. die senkrecht zur Strömung stehende Fläche des Körpers ist. Die senkrecht stehende Fläche entspricht dem größten Querschnitt des Körpers quer zur Strömungsrichtung.

Bei der Anwendung von Formel 4 treten in der realen Situation ähnliche Probleme auf wie bei Formel 7. Die Stirnfläche kann sich verändern, ebenso wie die Reynoldszahl, da sie neben der Oberflächengüte von der Form des Körpers abhängig ist. Die Form des menschlichen Körpers kann durch Veränderungen der Haltung variabel sein. Daraus folgend verändert sich die Stirnfläche, damit die Reynoldszahl und folgend der Strömungswiderstand.

Daher müsste die Vermessung der Stirnfläche und der Form konsequenterweise permanent sein. Die Anwendung der Formel zur Berechnung des Widerstandes eines hinreichend flexiblen Körpers, wie des menschlichen, ist deshalb nur mit extrem hohen Aufwand und komplexen Rechenmodellen möglich.

Stattdessen kann der passive Widerstand gemessen werden. Es erscheint dabei naheliegend, durch passives Ziehen bei gegebener Geschwindigkeit den Zugwiderstand zu ermitteln. KARPOVICH (1939), HOLMÉR (1974) untersuchten dazu Schwimmer, die sie an der Wasseroberfläche schleppen ließen. Sie fanden einen passiven Widerstand von $F_{D p}=29 \cdot v^{2}$. 


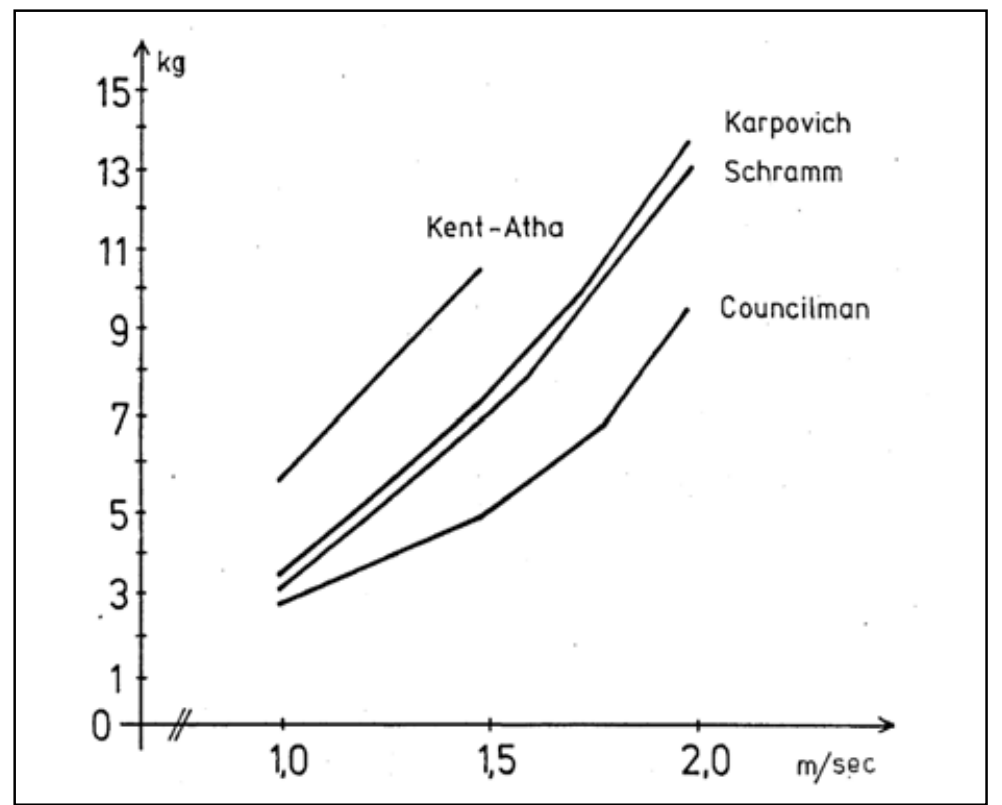

Abbildung 5: $\quad$ Widerstandswerte $\mathrm{F}_{\mathrm{Dp}}$ in kg von geschleppten Schwimmern bei verschiedenen Geschwindigkeiten (geändert nach Clarys/Jiskoot), UNGERECHTS $(1978,67)$.

UNGERECHTS (1978) verglich Untersuchungsergebnisse verschiedener Autoren, die an passiv geschleppten Schwimmern gewonnen wurden. Er stellte fest, dass die Kurven in allen Untersuchungen von Counsilman, Schramm und Karpovich parabolisch verlaufen, mit der Ausnahme der Untersuchung von Kent-Atha, bei der der Verlauf linear ist. Mit den gewonnen Ergebnissen sei zumindest der zu überwindende Widerstand festgestellt. Der parabolische oder polynomische Verlauf ist auf Grund der quadratischen Auswirkung der Geschwindigkeit als v² anzunehmen.

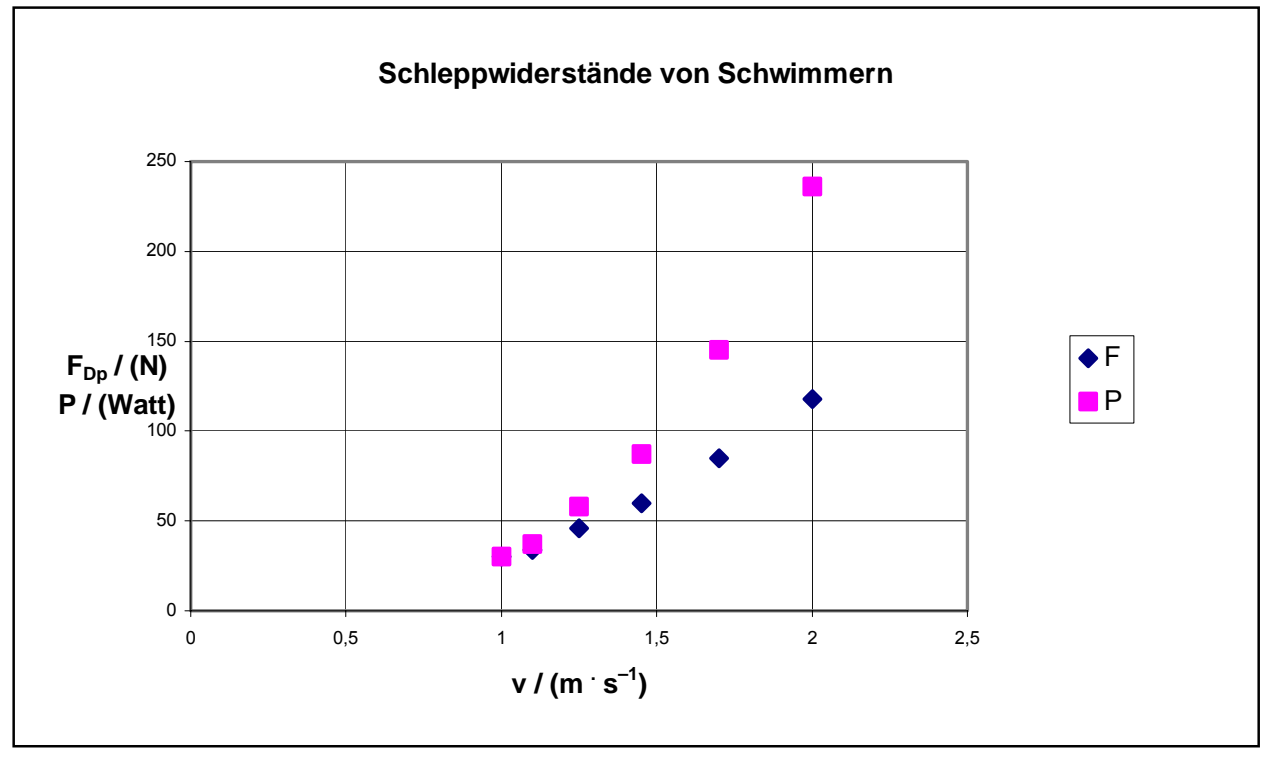

Abbildung 6: Schleppwiderstände von Schwimmern, modifiziert nach BINNER (1977, 467). 
Die von BINNER (1977) gemessenen Kraftwerte für den passiven Widerstand $F_{D p}$ von Schwimmern bei unterschiedlichen Geschwindigkeiten bestätigen die Aussagen von UNGERECHTS (1978) über die Ergebnisse von Counsilman, Schramm und Karpovich. Dazu wurden Schwimmleistungen in Watt von 30 bis 87 Watt, auf Basis des passiven Schleppens, errechnet.

Der Feststellung von HOLLANDER $(1994,386)$ und JAHNS $(2000,38)$ folgend, divergieren jedoch bei Schwimmern aktiver und passiver Wasserwiderstand.

Begründet ist diese Feststellung in dem Unterschied zwischen dem passiven Schleppen und dem Verhalten beim aktivem Schwimmen. Die optimale Schlepplage kommt beim Schwimmen nicht vor. Kraulschwimmer rotieren um die Körperlängsachse, der Kopf wird zur Atmung seitlich gedreht, Arme und Beine bewegen sich. Aus diesem Grund können die ermittelten Werte nur als Näherung an die realen Werte verstanden werden. Sie erscheinen aber hinreichend genau, zumindest so lange, bis geeignetere Verfahren zur Verfügung stehen.

Beim Schwimmtauchen mit Flossen sind in Schleppversuchen ermittelte Daten eine bessere Approximation an die reale Situation, da der Taucher sich nur mit den Flossen fortbewegt. Die Arme werden dabei ruhig in Vorhalte genommen und die Rotation des Körpers und die Seitwärtsbewegung des Kopfes finden nicht statt. Die Haltung entspricht der Körperhaltung beim sportlichen Streckentauchen in Apnoe ebenso wie auch bei der Benutzung von Pressluftflaschen.

Die Bestimmung des dynamischen oder aktiven Widerstandes ist auf direktem Wege nur mit großem Aufwand möglich. Auf den dynamischen Widerstand wird unter Punkt 1.9 eingegangen.

\section{6 Über die arbeits- und sportmedizinische Spiroergometrie}

Der Hauptinhalt der sportmedizinischen Spiroergometrie liegt in der Erfassung von Daten, mit deren Hilfe sich der Ökonomisierungsgrad von Herz-Kreislauf-, Atmungsund Stoffwechselprozessen auf differenzierten Leistungsstufen bestimmen lässt. Ein wesentlicher leistungsbestimmender Faktor ist die aerobe Kraftausdauer (aKa).

Ergebnisse lassen sich aus spiroergometrischen Leistungstests ablesen. Dazu müssen die Untersuchungskriterien exakt festgelegt werden. Danach ist die Zielstellung, alle benötigten, interessierenden oder relevanten physiologischen Antwortreaktionen zu bestimmen. Diese sollen dann auf die Eingangsgröße (bio-)mechanischer Leistung bezogen werden. Dazu ist eine physikalisch exakte Vorgabe, bzw. Bestimmung der Eingangsgröße notwendig. Darüber hinaus müssen die vom Sportler erbrachten Leistungen sportartspezifisch sein und die reale Belastungsstruktur simulieren oder reproduzieren.

Folgerichtig ist die aerobe Kraftausdauer (aKa) diejenige mechanische Leistung in Watt, die ein Proband unter den Bedingungen eines spiroergometrischen Stufentests, 
bedingt durch seine individuelle anaerobe-aerobe Stoffwechsellage, zu erbringen in der Lage ist.

STEGEMANN $(1991,261)$ und LATHAN $(1981,26)$ weisen auf den Zusammenhang hin, der zwischen dem Aussagewert der Leistungsdiagnostik und dem Abbildungsmaß typischer sportartspezifischer Bewegungsinhalte besteht. Das heißt, dass sich die Relevanz der funktionellen Messgrößen mit dem Grad der Erfassung und Reproduktion des sportartspezifischen Bewegungsablaufes erhöht. Dabei gilt, dass, je präziser die sportliche Technik mit den Belastungsvorrichtungen dargestellt werden kann, um so genauer wird auch die aerobe Kraftausdauer bestimmt werden können.

\subsubsection{Ermittlung des Energieumsatzes}

Der Energieumsatz kann mit zwei Methoden ermittelt werden, deren Ausgangspunkt identisch ist. Bei beiden Methoden werden Anfangs- und Endprodukt einer Reaktion gegenübergestellt. Bei der direkten Kalorimetrie ist das die nach außen abgegebene Wärmemenge. Die indirekte Kalorimetrie basiert auf der Gegenüberstellung des verbrauchten Sauerstoffs und der Kohlendioxidabgabe. Korrekt angewendet müssen direkte und indirekte Kalorimetrie zu gleichen Ergebnissen führen.

\subsubsection{Direkte Kalorimetrie}

Es wird die gesamte Wärme, die durch Leitung, Strahlung oder Verdunstung abgegeben wird, gemessen. Dieses Verfahren ist aufwendig und wird deshalb nur für spezielle Fragestellungen eingesetzt.

\subsubsection{Indirekte Kalorimetrie}

Die indirekte Kalorimetrie basiert darauf, dass eine stöchiömetrische Beziehung zwischen dem verbrannten Nährstoff und dem dabei verbrauchten Sauerstoff sowie dem abgegebenen Kohlendioxid besteht. Daraus folgt, dass das kalorische Äquivalent nicht eine konstante Größe ist, sondern davon abhängt, welcher Nährstoff verbrannt wird. Unter Vernachlässigung des Eiweißstoffwechsels kann das Äquivalent offensichtlich zwischen 19 bis $21,3 \mathrm{~kJ} / / \mathrm{O}_{2}$ schwanken.

Das Verhältnis von abgegebenem $\mathrm{CO}_{2}$-Volumen $\dot{V} \mathrm{CO}_{2}$ und aufgenommenem $\mathrm{O}_{2}$ Volumen $\dot{V} \mathrm{CO}_{2}$ bezeichnet man als den Respiratorischen Quotienten (RQ).

$$
R Q=\frac{\dot{V} \mathrm{CO}_{2}}{\dot{\mathrm{VO}}}
$$

Fehlerquellen können metabolischer oder respiratorischer Art sein. Wird z.B. Kohlehydrat in Fett umgebaut, so wird $\mathrm{O}_{2}$ frei, das deshalb weniger aufgenommen wird. Dadurch kann der RQ höher als 1 liegen BRAUER/GOTTSCHALK $(1996,138)$. 
Im Hungerzustand, dem umgekehrten Fall, kann der RQ ungewöhnlich niedrige Werte annehmen. Jede Hyperventilation, die schon durch ein ungewohntes Atemmundstück einer Messeinheit ausgelöst wird, kann zu einer Verschiebung des $\mathrm{PCO}_{2}$ im Blut führen und damit zu einer Verschiebung des $R Q$ zu höheren Werten. Gleiches gilt für erhöhte Atemarbeit, wie sie z.B. durch einen Lungenautomaten/Atemregler, wie er im Tauchsport benutzt wird, provoziert werden kann.

Die Einheit für die Kraft $F$ ist Newton $(N)$. Kräfte sind dabei Vektorgrößen, denen Betrag und Richtung zugeordnet werden.

Die Arbeit W ist das Produkt aus Kraft F und Weg s in Wegrichtung:

$$
W=F \cdot s
$$

Die Einheit ist $\mathrm{N} \cdot \mathrm{m}=\mathrm{Nm}=$ Joule. Kraft- und Wegrichtung müssen zur korrekten Anwendung dieser Formal identisch sein, da sonst die Arbeit über das Integral bestimmt werden muss.

\section{$1.7 \quad$ Belastung}

Im sportmedizinischen Sinn wird unter Belastung eine körperliche Aktivität zur Auseinandersetzung des Organismus mit Kräften unter statischen oder dynamischen Bedingungen verstanden. Diese geht über das physische Beanspruchungsmaß der körperlichen Ruhe hinaus und stimuliert den Organismus partiell oder in seiner Gesamtheit (Stoffwechsel, Halte-, Stütz- und Bewegungssystem, Nervensystem, kardiopulmonales System, etc.) durch Überschreiten einer bestimmten Reizschwelle. Die Belastung ist charakterisiert durch ihren Umfang und ihre Intensität (vgl. BRAUER/GOTTSCHALK 1996, 37).

ULMER $(1983,602)$ definiert Belastung als „eine vorgegebene Anforderung, die von äußeren Bedingungen, nicht aber vom betroffenen Individuum abhängt“.

Folgt man beiden Definitionen, muss man, damit man die Belastung definieren kann, zuerst das Beanspruchungsmaß der körperlichen Ruhe ermitteln. Um dann die zusätzliche Stimulation des Körpers bestimmen zu können, müssen die Bedingungen, welche die zusätzliche Belastung ergeben, exakt beschrieben werden.

\subsubsection{Belastung beim Tauchen}

Beim Tauchen bewegt sich der menschliche Körper im Medium Wasser und der Begriff Tauchen ist die Sammelbezeichnung für alle arbeitstechnischen, leistungs-, freizeit-, und breitensportlichen Aktivitäten, die im Bewegungsraum unterhalb der Wasseroberfläche ausgeführt werden. 
Die Fortbewegung unter Wasser wird mit Flossen realisiert. Das bedeutet, dass der Schwimmtaucher mit Hilfe von Flossen, unter Aufrechterhaltung des hydrostatischen Gleichgewichts (Tarierung), bei wechselnden Bedingungen, wie vertikalen und horizontalen Strömungen, sowie Widerstandsänderungen auf Grund von Veränderungen der Ausrüstung oder der Wasserlage Vortrieb erzeugen und möglicherweise parallel mit den Händen weitere Tätigkeiten ausführen muss.

Die Belastung des Schwimmtauchers ergibt sich aus einer Vielzahl von qualitativ und quantitativ unterschiedlichen Leistungen, die in verschiedenen Situationen erbracht werden müssen. Diese ist darüber hinaus von individuellen, sportartspezifischen und allgemeinen konditionellen Leistungsvoraussetzungen abhängig. Die wesentliche Leistung muss erbracht werden, um sich unter Wasser vorwärts zu bewegen. Dazu kommen Flossen zum Einsatz, welche in unterschiedlichen Variationen in Aufbau, Form, Design, Härte, Schwingungsverhalten etc. zur Verfügung stehen.

Der oben angeführten Definition von $\operatorname{ULMER}(1983,602)$ folgend, treten Probleme auf, da es Schwierigkeiten bereitet, die äußeren Bedingungen in ihrer Wirkung klar zu definieren und die Angriffspunkte der Kraftvektoren und der Richtung zu bestimmen. Diese Vorgehensweise wäre aber dennoch die mathematisch genaueste Methode und somit die eigentlich wünschenswerte Vorgehensweise (vgl. JAHNS 2000, 50 u. 66).

Beim Schwimmtauchen wie auch beim Schwimmen weniger jedoch beim Radfahren und Laufen wird immer wieder versucht die Belastung quantitativ über die Geschwindigkeit zu definieren. Diese Vorgehensweise ist jedoch ungenau, da die mechanische Leistung in Bezug zur Geschwindigkeit nicht direkt festgestellt werden kann, da bei

$$
P_{\text {mech }}=F \cdot v
$$

die zur jeweiligen lokomotorischen Geschwindigkeit v gehörende Kraft F nicht messbar ist.

Verfahren wie die Fahrrad- oder Laufbandergometrie sind relativ sicher zu beschreibende Verfahren, da hier Kraftvektoren, Wegstrecken und Zeiten gemessen werden können und sich daraus die mechanische Arbeit bzw. Leistung berechnen lässt. Im Verhältnis zur gleichzeitig aufgenommenen Energie kann auf den Wirkungsgrad geschlossen werden.

Im Wasser treten jedoch keine isolierten mechanischen Einzelkräfte auf. Vielmehr ist die Vortriebskraft eine Resultierende aus einer Summe von Einzelkräften in Schwimmrichtung, da die Vortriebs- und Widerstandskräfte ausschließlich dynamische Kräfte sind, welche aus der Bewegung als Folge von Strömungswiderständen entstehen. Sie sind daher in Betrag und Richtung nicht direkt messbar.

Im Bereich des Sporttauchens beschränkt sich die mit den oberen Extremitäten zu erbringende Leistung auf das ruhige Halten der Hände. Diese werden nur bewegt, um die Instrumente ablesen zu können oder einfache Handgriffe an der Ausrüstung auszuführen (Bedienung des Inflators, Luftablassen etc.). Bei Strömung kann 
Haltearbeit geleistet werden, wenn sich der Schwimmtaucher an einem Seil festhalten oder entlang ziehen muss.

Bei Unterwasserarbeiten, wie sie in der Berufstaucherei erledigt werden müssen, kann eine große Bandbreite von Belastungen entstehen, welche von hohen koordinativen Anforderungen bei Nullsicht ohne physische Belastung der oberen oder unteren Extremitäten, z.B. bei der Montage von Kleinteilen, über hohe körperliche Belastung der oberen Extremitäten, z.B. beim Hämmern oder Schleifen, bis hin zu starker Belastung des gesamten Organismus, z.B. beim Abtauchen einer Strecke im Rahmen einer Unterwassersuche, reichen.

Daneben entstehen weitere Belastungen durch Lärm wegen der erhöhten Schallgeschwindigkeit im Wasser, durch nicht vorhandene Sicht und durch Kälte. Auf die psychischen Belastungen, die z.B. im Rahmen der Suche nach einer vermissten Person auftreten können, sei hingewiesen.

\section{$1.8 \quad$ Leistung}

In der Theorie des Sports wird mit dem Begriff der Leistung eine Vielzahl von Kriterien und Parametern je nach Betrachtungsweise und Erkenntnisinteresse der Autoren in Verbindung gebracht. In der sportmedizinischen und arbeitsmedizinischen Diagnostik ist die körperliche Leistungsfähigkeit eng auf die Betrachtung der kardiopulmonalen Funktionsgrößen gerichtet. Diese werden in Beziehung zu den erbrachten Leistungen gesetzt und ermöglichen eine Bewertung und Einschätzung der Leistungsfähigkeit. Dazu müssen die physikalischen Eingangsgrößen ebenso wie die veränderten körperlichen Umsatzgrößen bestimmt werden, welche die Antwortreaktion des Körpers ausmachen.

Als Eingangsgröße für die leistungsphysiologische Untersuchung gilt die vom Sportler bewältigte Belastung, also die in einer bestimmten Zeit $t$ verrichtete mechanische Arbeit $W$ als Produkt aus einer an der Wegstrecke s entlang wirkende Kraft $F$, also die mechanische Leistung $P_{\text {mech }}$ in Watt:

$$
\begin{gathered}
\text { Leistung = Arbeit } \cdot \text { Zeit }^{-1}=\text { Kraft } \cdot \text { Weg } \cdot \text { Zeit }^{-1}=\text { Kraft } \cdot \text { Geschwindigkeit } \\
P_{\text {mech }}=W \cdot t^{-1}=F \cdot s \cdot t^{-1}=F \cdot v \\
(\text { Watt })=\left(N \cdot m \cdot s^{-1}\right)=\left(\text { Joule } \cdot s^{-1}\right)
\end{gathered}
$$

v symbolisiert dabei die lokomotorische Geschwindigkeit des Sportlers.

Bei sportlichen Bewegungsabläufen ist $P_{\text {mech }}$ nur in den Fällen genau messbar, in denen präzise Daten erhoben werden können. Dabei sei auf die unterschiedliche Genauigkeit von Fahrrad-, Ruder-, Kanu- und Handkurbelergometer etc. hingewiesen. Auf dem Laufband behilft man sich mit der Angabe der lokomotorischen 
Geschwindigkeit v als Leistungskennwert. Bei einigen Untersuchungen zum Vortrieb im Wasser (SCHLAG AUF SCHLAG 1997, SCHUBWERKZEUGE 2000, ANTRIEBSPADDEL 2002, FOURTRIEB 2000) wurde ähnlich verfahren. Die nach o.a. Gleichung zur Bestimmung der mechanischen Leistung erforderlichen Kraftgrößen sind in diesen Fällen nicht direkt messbar, jedoch zur exakten Bewertung der physiologischen Antwortreaktion erforderlich. Die lokomotorische Geschwindigkeit darf demgegenüber nicht mit der Leistung in Watt gleichgesetzt werden. Als Folge sind die gewonnenen Ergebnisse nicht mehr eindeutig zuzuordnen.

Der Organismus reagiert auf höhere Belastungen u.a. durch Erhöhung der Stoffwechselaktivitäten. Dieses zeigt sich in der Erhöhung der Sauerstoffaufnahme, der Herzschlagfrequenz sowie der Veränderung biomechanischer Messgrößen. Der Sauerstoffaufnahme $\dot{V} \mathrm{O}_{2}$ kommt eine zentrale Bedeutung zu. Aufgrund thermodynamischer Gesetzmäßigkeiten ist sie das Maß für die vom Körper aufgewandte (Brutto-)Leistung. Mit der Methode der indirekten Kalorimetrie kann aus der Sauerstoffaufnahme unter Berücksichtigung des kalorischen Äquivalentes k direkt auf die Bruttoleistung geschlossen werden (vgl. STEGEMANN 1984, 57-60).

$$
P_{\text {brutto }}=k \cdot \dot{V} \mathrm{O}_{2}
$$

In die Bruttoleistung gehen die mechanische Leistung und kalorische Leistung (Wärmeabgabe) ein, so dass der Zusammenhang der drei Leistungsformen mit:

$$
P_{\text {brutto }}=P_{\text {mech }}+P_{\text {kal }}
$$

beschrieben werden kann. Würde der Begriff „Leistung“ mit dem Begriff „sportliche Leistung" gleichgesetzt werden, könnte die physiologische Antwortreaktion des menschlichen Organismus nicht mehr exakt bestimmt werden. Es ist also nicht korrekt die lokomotorische Geschwindigkeit synonym für den Begriff „Leistung“ zu setzen. Daraus ergibt sich, dass entsprechend der Methode der indirekten Kalorimetrie die Bruttoleistung $P_{\text {brutto }}$ der ermittelte gesamte Energieaufwand und unter der Nettoleistung $P_{\text {mech }}$ nur die mechanische Ergometerleistung $z u$ verstehen ist. Die kalorische Leistung $P_{\text {kal }}$ setzt sich aus mechanischen Energieverlusten und anderen Verlusten wie z.B. Wärme zusammen. In die kalorische Leistung $P_{\text {kal }}$ fließt auch die Atemarbeit, die bei der Spiroergometrie unter Wasser durch den Atemwiderstand des Lungenautomaten entsteht, insbesondere beim Einsatz der vom Autor konstruierten umgebungsdruckgesteuerten Ausatemventile, ein.

Für die arbeitsmedizinische Untersuchung von Berufstauchern sind entsprechend den europäischen Richtlinien für Berufstaucher die Testresultate der Tauchtauglichkeitsuntersuchung in MET entsprechend $\dot{V} \mathrm{O}_{2 \max }$ relativ zum Körpergewicht pro Minute $\left(\mathrm{ml} \mathrm{O} \mathrm{O}_{2} \cdot \mathrm{kg}^{-1} \cdot \mathrm{min}^{-1}\right)$ anzugeben EUROPEAN DIVING TECHNOLOGY COMMITEE $(2003,25)$. Nach diesen Richtlinien müssen Berufstaucher in der Lage sein, ein $\dot{V} \mathrm{O}_{2 \max }$ von $45 \mathrm{ml} \mathrm{O} \mathrm{O}_{2} \cdot \mathrm{kg}^{-1} \cdot \mathrm{min}^{-1}$ leisten $\mathrm{zu}$ können. Dabei müsste der theoretisch errechnete Maximalwert sogar über diesem Wert liegen, um ein sicheres Arbeiten unter Wasser gewährleisten zu können (vgl. WENDLING et al. 2001, 193). 


\section{$1.9 \quad$ Der dynamische Widerstand}

Während der passive Widerstand relativ einfach durch passive Schleppversuche zu bestimmen ist, kann dieses Verfahren beim aktiven Schwimmen nicht angewendet werden. In der Literatur findet sich kein Messverfahren zur direkten Bestimmung der Widerstandkraft F beim aktiven Schwimmen. Das oben beschriebene CFD-Verfahren wäre eine geeignete Methode, um die sich ändernde Stirnfläche eines Schwimmers oder Schwimmtauchers permanent bestimmen zu können. Die Berechnungen dazu würden allerdings sehr aufwendig und zeitraubend sein. Gleichzeitig ist festzustellen, dass der Widerstandsbeiwert nicht ermittelt werden kann, da die Bedingungen, um inn zu messen, beim aktiven Schwimmen nicht geschaffen werden können. Die Gleichung (4) gilt überdies hinaus nur für laminare Strömungen. Beim aktiven Schwimmen treten aber vor allem turbulente Strömungen auf.

NIKLAS (1989, 54ff) stellte für das Schwimmen und Schwimmtauchen die Methode der Zusatzkraftbeaufschlagung vor. Hierbei wird ein Proband im Strömungskanal bei konstanter Geschwindigkeit mit Zusatzkräften, die in Vortriebsrichtung wirken be- und entlastet. Mit dieser Methode lässt sich durch Extrapolation der Regressionsgeraden auf die Ruhesauerstoffaufnahme die mechanische Leistung eines Probanden hinreichend genau ermitteln. Von der Sauerstoffaufnahme kann direkt, unter Berücksichtigung des kalorischen Äquivalentes k, auf die Bruttoleistung geschlossen werden und für einen biologischen Organismus der Wirkungsgrad in Analogie zu physikalischen Systemen als Verhältnis von mechanischer Leistung zu Bruttoleistung definiert werden.

Da im Falle der Zusatzkraftbeaufschlagung eines aktiven Probanden bei v = konstant gilt

$$
\Delta P_{\text {mech }} \sim \Delta \dot{V} \mathrm{O}_{2}
$$

$\Delta \mathrm{P}_{\text {mech }}$ und $\Delta \mathrm{F} \cdot \mathrm{v}$ sind exakt bekannt. Auf Grund der Verbindung gemäß Gleichung (13) und Gleichung (14), die als hinreichend exakt gelten, kann in einem Test mit mehreren Zusatzkräften die mechanische aktive Leistung eines Probanden durch Extrapolation der Regressionsgeraden auf die Ruhesauerstoffaufnahme hinreichend genau ermittelt werden. Eine Abweichung der Messpunkte von der Geradengleichung schrieb NIKLAS folgenden Ursachen zu:

1. Die Zusatzkräfte waren zu groß gewählt

2. Die sportliche Technik des Probanden war über die Versuchsdauer nicht stabil.

3. Der Proband erreichte sein $\dot{V} \mathrm{O}_{2 \max }$.

4. Die Stufendauer war zu kurz.

5. Stochastische Messfehler konnten zur Verringerung des

Bestimmtheitsmaßes und zur Verbreiterung des Konfidenzintervalls führen. 
NIKLAS $(1989,55)$ stellte fest, dass sich bei einem Bestimmtheitsmaß $R^{2}<0,96$ die hydrodynamische Widerstandskraft über ein Kurvenanpassungsverfahren als Polynom abzubilden ist, indem die erste Ableitung im Punkt $\left(\dot{V} \mathrm{O}_{2} ; \Delta \mathrm{F}=0\right)$ gebildet wird. Die Anstiegsgerade wird im Schnittpunkt der Arbeits- $\dot{V} \mathrm{O}_{2}-$ Achse auf die Ruhesauerstoffaufnahme extrapoliert. Für alle Fälle $R^{2}>0,95$ für eine Gerade seien die mittels beider Verfahren errechneten Beträge identisch.

Bedingung ist, dass ein möglichst rückwirkungsfreies Verfahren gewählt wird. Dazu sind die Zusatzkräfte in der Wirkrichtung der Vortriebs- und Widerstandskräfte anzubringen.

\section{$1.10 \quad$ Wirkungsgrad}

Analog zu technisch-physikalischen Systemen kann aus dem Verhältnis von $P_{\text {mech }}$ und $P_{\text {brutto }}$ der Bruttowirkungsgrad $\eta$ bestimmt werden. Entsprechend dem Verfahren der indirekten Kalorimetrie ist der Bruttowirkungsgrad $\eta_{\text {brutto }}$ definiert aus dem Verhältnis von Leistung zu Gesamtumsatz:

$$
\eta_{\text {brutto }}=\frac{P_{\text {mech }}}{P_{\text {brutto }}}
$$

Gelingt es, die Wärmeabgabe eines Sportlers während des Beobachtungszeitraums unter definierter Belastung zu bestimmen, kann der Wirkungsgrad auch als:

$$
\eta_{\text {brutto }}=1-\frac{P_{\text {kal }}}{P_{\text {bruto }}}
$$

bestimmt werden. NIKLAS $(1989,14)$ weist darauf hin, dass im Schwimmen bereits entsprechende Untersuchungen stattgefunden haben.

Zusätzlich kann der Nettowirkungsgrad $\eta_{\text {netto }}$ bestimmt werden. Der Nettowirkungsgrad $\eta_{\text {netto }}$ ist definiert als Quotient aus Leistung und dem dafür aufgewendeten Arbeitsumsatz:

$$
\eta_{\text {netto }}=\frac{P_{\text {mech }}}{P_{\text {neto }}}
$$

In Untersuchungen beim Tauchen mit Atemregler muss in $P_{\text {kal }}$ nicht nur die Wärmeabgabe, sondern auch die zusätzlich durch den Atemregler verursachte Atemarbeit berücksichtigt werden.

Der Wirkungsgrad beim Schwimmen wird mit $0,5 \%$ bei Ungeübten bis $8 \%$ bei Spitzenschwimmern angegeben STEGEMANN (1991, 250). NIKLAS et al. (1988) nennt einen Wirkungsgrad von $5 \%$ für Taucher und GOFF (1957) stellt einen Wirkungsgrad von $1,2 \%$ bis $5,6 \%$ an untrainierten Tauchern fest. 
Studienziel sind Antworten auf die folgenden Fragen:

1. Welche Widerstände müssen Schwimmtaucher unter Wasser überwinden?

Dazu soll der passive Widerstand, den Schwimmtaucher mit wechselnden Ausrüstungsgegenständen haben, festgestellt werden. Von besonderem Interesse ist, ob methodisch die Möglichkeit besteht, sich dem Aspekt des dynamischen Widerstandes anzunähern und plausible Ergebnisse vorzustellen.

2. Welche Leistung müssen Schwimmtaucher zur Überwindung von Widerständen unter Wasser erbringen?

Dazu soll eine Methode zu Anwendung kommen, welche die Belastungssituation unter Wasser exakt abbildet. Die Methode soll objektivierbar, validierbar, standardisierbar und reliabel sein. In ihr sollen die Belastungsparameter wie Widerstandskräfte ebenso erhoben werden können wie die Stoffwechseldaten der Probanden.

3. Kann ein Ventil konstruiert werden, mit dem die für die spiroergometrische Untersuchung benötigte Ausatemluft umgebungsdruckgesteuert zur Wasseroberfläche abgeleitet werden kann? Wird ein solches Ventil den Anforderungen der EN 250 entsprechen und seine Einsatztauglichkeit beweisen?

\section{$2.1 \quad$ Studiendesign, Zielparameter}

Es handelt sich um eine Pilotstudie zur Grundlagenentwicklung an Sporttauchern, mit denen jeweils 2 Versuche durchgeführt werden:

- Widerstandsmessungen im Schwimmkanal mit variierenden Ausrüstungsgegenständen bei wechselnden Strömungsstärken.

- Belastungsspiroergometrie im Schwimmkanal bei festen Strömungswerten und variabler Zusatzkraftbeaufschlagung.

Zielparameter ist der Widerstand $F$ bezogen auf unterschiedliche Ausrüstungskonfigurationen, Sauerstoffaufnahme und respiratorischer Quotient. 


\subsection{Fragestellung und Hypothesen}

1. Es werden turbulente Strömungsverhältnisse im Strömungskanal erwartet. Deshalb wird die reale Strömungssituation am Tauchpunkt der Probanden ermittelt. Es wird erwartet, dass diese realen Strömungsgeschwindigkeiten nicht mit den voreingestellten Werten übereinstimmen.

2. Es wird erwartet, dass der passive Widerstand der Probanden sich in Abhängigkeit zu den getragenen Ausrüstungsgegenständen ändert. Dabei könnte der Einfluss von Flossen auf die Kraftwerte gering sein, da diese sich im wesentlichen im Strömungsschatten der Probanden befinden. Die Differenz der Kraftwerte von Auftriebskörpern wird als erheblich vermutet, da diese die Stirnfläche der Probanden erheblich vergrößern.

3. Die Probanden tauchen mit verschiedenen Flossen, um einem festgelegten Widerstand widerstehen zu können. Es ist zu erwarten, dass sie dazu, bezogen auf die Flossen, unterschiedliche Leistungen erbringen müssen. Mit den Ergebnissen können Aussagen über die Eignung der Flossen getroffen werden. Als Bewertungsgrundlage dienen Netto- und Bruttowirkungsgrad, Sauerstoffaufnahme, der Gesamtumsatz sowie MET.

4. Auf Basis der Sauerstoffaufnahme auf den Belastungsstufen, die mit der Methode der Zusatzkraftbeaufschlagung (vgl. NIKLAS et al. 1988) gewonnen werden, scheint es möglich über die Regression der Geraden auf die Abszisse mit $\dot{V} \mathrm{O}_{2}=0$ den Wert des dynamischen Widerstandes plausibel und hinreichend genau zu ermitteln. Dieser wird größer als der ermittelte passive Widerstand erwartet. Er könnte hypothetisch auf Grund der insgesamt günstigen Strömungsform des Schwimmtauchers in geringem Abstand oberhalb des passiven Widerstandes liegen.

5. Die Untersuchungsmethode erscheint zur Leistungsdiagnostik geeignet und kann im Rahmen der Tauchtauglichkeitsuntersuchung eingesetzt werden. 


\subsection{Probanden}

Als Probanden stehen zwei Leistungsflossenschwimmer zur Verfügung. Proband $A$ ist $182 \mathrm{~cm}$ groß und $78 \mathrm{~kg}$ schwer und Proband $B$ ist 175 groß und $68 \mathrm{~kg}$ schwer. Beide Probanden sind 16 Jahre alt und Mitglied im Jugendkader Flossenschwimmen des Tauchsportlandesverbandes Niedersachsen.

\subsection{Ort der Versuche}

Alle Untersuchungen finden im Schwimmkanal der Elbeschwimmhalle in Magdeburg statt. Im Schwimmkanal bewegt sich der Schwimmtaucher in einem horizontalen Kreisstrom, welcher durch Impeller erzeugt wird. Der Bereich, in dem getaucht werden kann, ist 1,40 m tief, 4,00 m lang und 3,00 m breit. Die Anströmgeschwindigkeit ist veränderbar. Der Taucher kann von der Seite durch ein Fenster beobachtet werden.

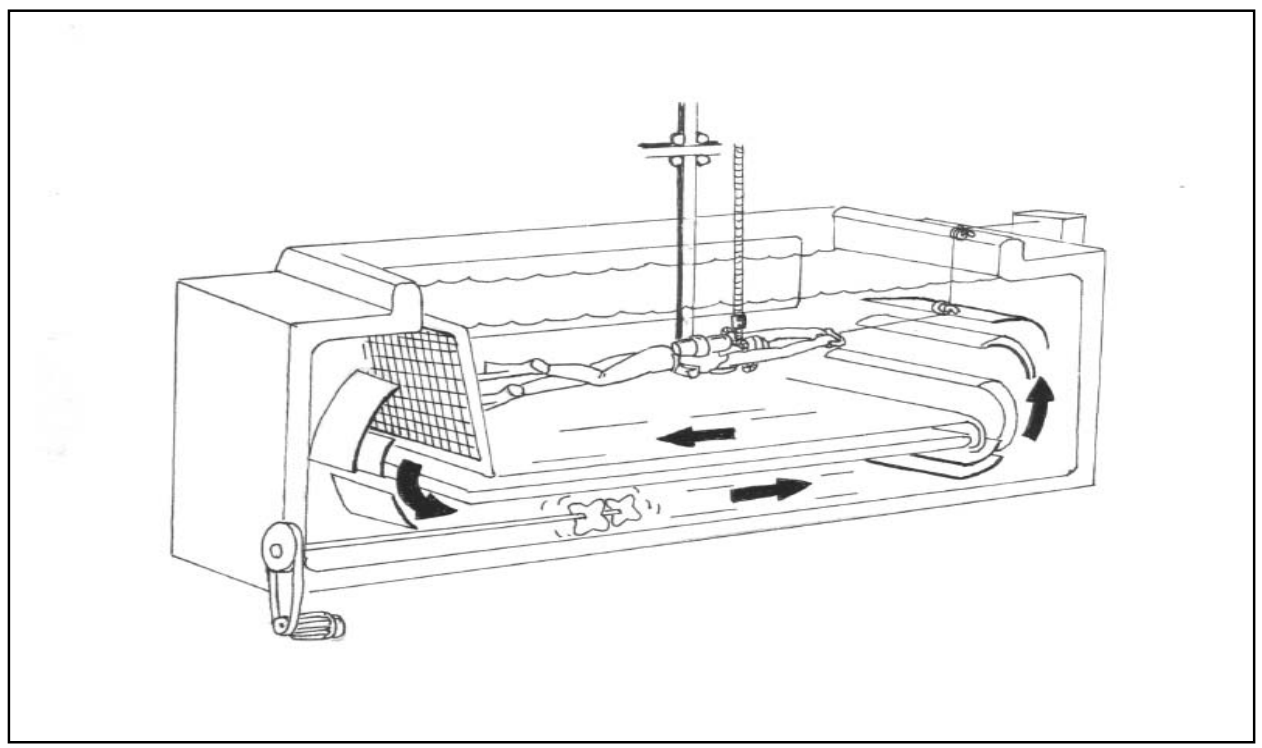

Abbildung 7: $\quad$ Der Schwimmkanal der Elbeschwimmhalle in Magdeburg. Eine ähnliche Konstruktion wurde von ÅSTRAND et al. (1972) benutzt. ALMELING (2004) Med-Habil. Göttingen (in Vorbereitung). Mit freundlicher Genehmigung des Autors.

\subsection{Methoden}

\subsubsection{Bestimmung der Strömungsverhältnisse im Kanal (Messreihe 1) und die Kalibrierung des Kraftaufnehmers}

In der ersten Messreihe werden die realen Strömungsgeschwindigkeiten im

Strömungskanal bestimmt, die bei den vorgegebenen Einstellungen an der 
Steuerungseinheit des Schwimmkanals im Wasser auftreten. Die Messungen werden mit 5 parallelen Impellern vorgenommen, welche in einer Tiefe von $8 \mathrm{~cm}$ und $47 \mathrm{~cm}$ jeweils vorne, in der Mitte und hinten im Kanal bei den o.a. Geschwindigkeitsstufen positioniert sind. Die ermittelten Daten sind Basis für die weiteren Berechnungen, in welche die Strömungsgeschwindigkeit einfließt.

Jeder Versuch wird in den folgenden Stufen absolviert, wobei sich die real im Strömungskanal gemessenen Geschwindigkeiten von denen, die am Kanal eingestellt werden, unterscheiden. Diese Differenzen sind in den entsprechenden Berechnungen berücksichtigt. Wenn von Stufe 1 oder Geschwindigkeit 1 etc. gesprochen wird, so wird immer auf die realen Daten Bezug genommen.

Die Auswahl der Geschwindigkeitsstufen ist von der Konstruktion des Strömungskanals vorgegeben. Diese Auswahl entspricht Geschwindigkeiten, welche für den Taucher in der Realität vorkommende Situationen abbilden. Als maximal erreichbare Geschwindigkeit für Flossenschwimmer im Sprint wird $3,5 \mathrm{~m} \cdot \mathrm{s}^{-1}$ angeführt, entsprechend 12,6 km $\cdot \mathrm{h}^{-1}$ VDST $(2004,1)$. PENDERGAST (2003 a, 64, 2003 b, 79) untersucht männliche Flossenschwimmer bei Geschwindigkeiten von 0,4 bis $0,9 \mathrm{~m} \cdot \mathrm{s}^{-1}$ und weibliche Flossenschwimmerinnen bei Geschwindigkeiten von 0,4 bis $0,7 \mathrm{~m} \cdot \mathrm{s}^{-1}$. In Untersuchungen im Strömungskanal der Zeitschrift TAUCHEN (1997, 107) wird von einer normalen Geschwindigkeit eines Tauchers von $0,4-0,5 \mathrm{~m} \cdot \mathrm{s}^{-1}$ ausgegangen, welche von HOFFMANN (2002) mit $0,5 \mathrm{~m} \cdot \mathrm{s}^{-1}$ bestätigt werden. $\mathrm{Er}$ merkt jedoch an, dass auch im Rahmen eines normalen Tauchgangs eine erhöhte physische Belastung auftreten kann. Konkret nennt er Schwimmen gegen starke Strömung, Schnorcheln mit kompletter Ausrüstung an bewegter Oberfläche und den Transport des Tauchpartners. Diese Belastungsspitzen lassen es sinnvoll erscheinen, die Messungen auch auf Geschwindigkeiten auszudehnen, die deutlich über den o.a. normalen Geschwindigkeitswerten liegen und mit den Stufen $0,9 \mathrm{~m} \cdot \mathrm{s}^{-1}, 1,2 \mathrm{~m} \cdot \mathrm{s}^{-1}$ und $1,5 \mathrm{~m} \cdot \mathrm{s}^{-1}$ berücksichtigt werden.

Es ist vor Beginn der Widerstandsmessungen zu überprüfen, wie schnell der Kraftaufnehmer auf Lastwechsel reagiert. Ausgehend von einer definierten Last ist die Formel zu bestimmen, mit der die erhobenen Werte umgerechnet werden müssen.

\subsubsection{Bestimmung der Strömungswiderstände der Probanden mit unterschiedlichen Auftriebskörpern (Messreihe 2)}

Die Messungen der Strömungswiderstände wird mit folgendem Geräteaufbau vorgenommen: Der Proband hängt an einem Griff, welcher mit dem Kraftnehmer verbunden ist, in der Strömung. Mit dieser Vorrichtung wird die Widerstandskraft $F_{w}$ aufgenommen, mit welcher er der Strömung widersteht. Die Daten werden an einen PC übertragen.

Bei den Versuchen befindet sich der Proband in einer optimalen, möglichst starren und über den Versuchsablauf möglichst konstant beibehaltenen Lage. 


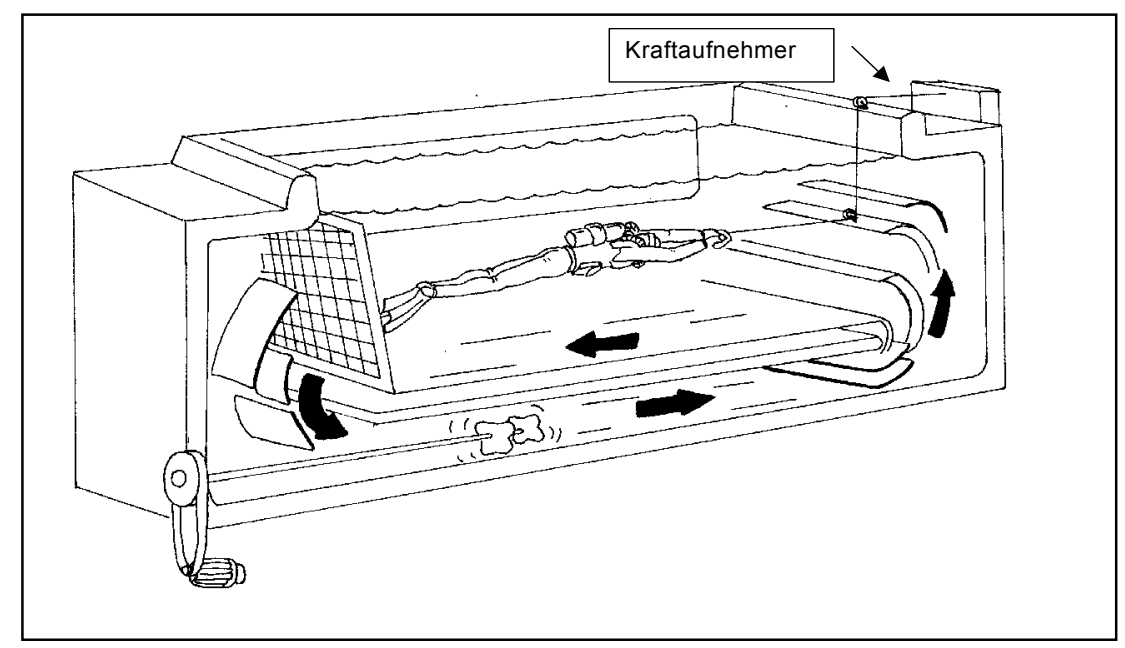

Abbildung 8: $\quad$ Der Versuchsaufbau für Messreihe 2.

Beide Probanden tragen als identische Ausrüstungsgegenstände ein DTG mit 10 Liter Inhaltsvolumen durch welches er mit Luft versorgt wird und Flossen des Herstellers Mares vom Typ Volo in der Größe: R.

Seiner Körpergröße angepasst trägt Proband A einen Anzug Größe 98, einen Bleigurt mit 6,5 kg Blei sowie Füßlinge Größe 43 und Proband B einen Anzug Größe 50, einen Bleigurt mit 5,5 kg Blei sowie Füßlinge Größe 41.

Die Unterschiede beim Anzug leiten sich aus der Konfektionsgröße der Probanden ab. Dazu muss die korrekte Bleimenge ermittelt werden. Mit der zu ermittelnden Bleimenge muss es den Probanden möglich sein, die Versuche mit luftleerem Tariermittel resp. Auftriebskörper austariert, also im hydrostatischen Gleichgewicht, durchzuführen. Daraus resultieren die o.a. unterschiedlichen Bleimengen für Proband $A$ und $B$.

Variiert wird der Ausrüstungsteil Auftriebsmittel. Beide Probanden werden mit dem System HUB, welches eine komplette Einheit aus Tariermittel und Atemregler darstellt, sowie der Kragenweste La Spirotechnique mit Westenflasche, dem Wing Jacket Zeagle und dem Spiro 1 Jacket ausgestattet. Außer beim HUB tauchen die Probanden mit einem Lungenautomaten Hersteller Sherwood Typ Brut mit Manometer, befestigtem Octopus und Inflatoranschluß.

Die ermittelten Mittelwerte der Widerstände je Strömungsgeschwindigkeit werden in polynomischen Funktionen 2. Grades dargestellt und je Proband und je Auftriebskörper verglichen.

Auf Basis der erhobenen Werten wird die Leistung errechnet, die benötigt wird, um der Strömung widerstehen zu können. 


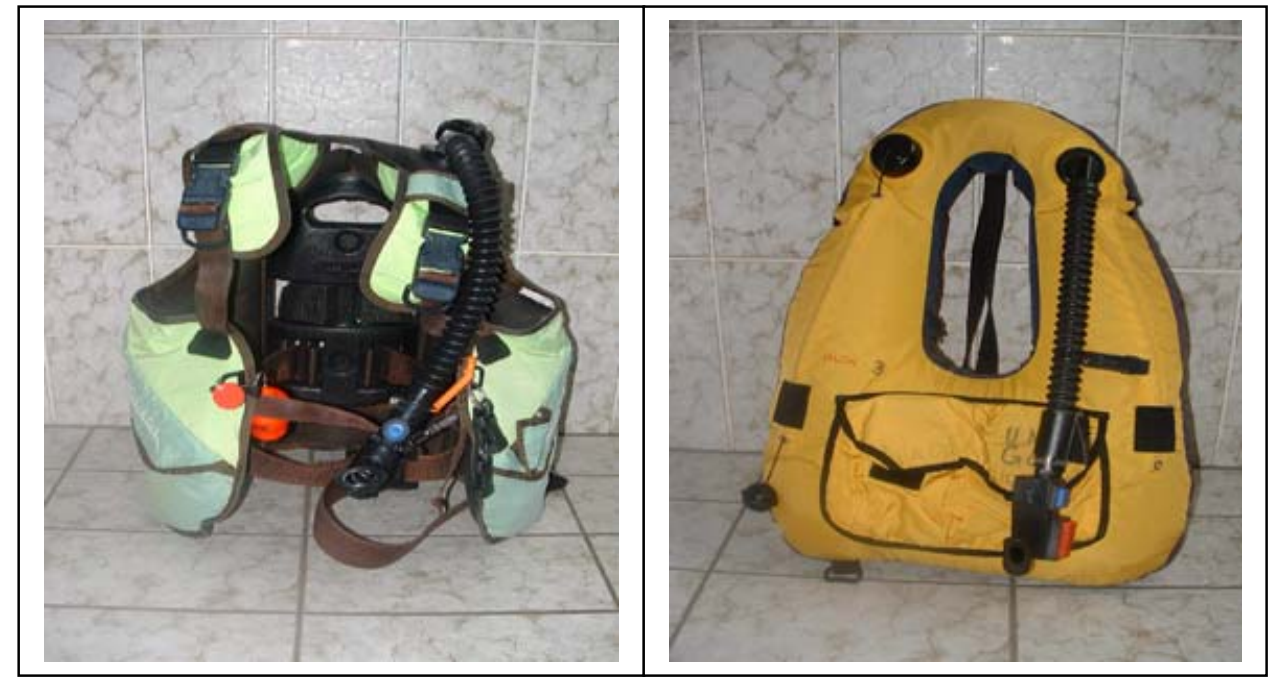

\begin{tabular}{|c|c|c|c|}
\hline & Bild links & & Kragenweste \\
\hline $\begin{array}{l}\text { Hersteller } \\
\text { Typ } \\
\text { Merkmale }\end{array}$ & $\begin{array}{l}\text { Spriotechnique } \\
1 \\
\text { Schnellablass rechts oben; } \\
\text { Inflator mit Schlauch rechts; } \\
\text { Octopushalter links. }\end{array}$ & $\begin{array}{l}\text { Hersteller } \\
\text { Typ } \\
\text { Merkmale }\end{array}$ & $\begin{array}{l}\text { Spriotechnique } \\
\text { unbekannt } \\
\text { Schnellablass rechts oben, } \\
\text { Inflator mit Schlauch rechts; } \\
\text { separater Schnellablass links; } \\
\text { Octopushalter und Westenflasche } \\
\text { ohne Abbildung. }\end{array}$ \\
\hline
\end{tabular}

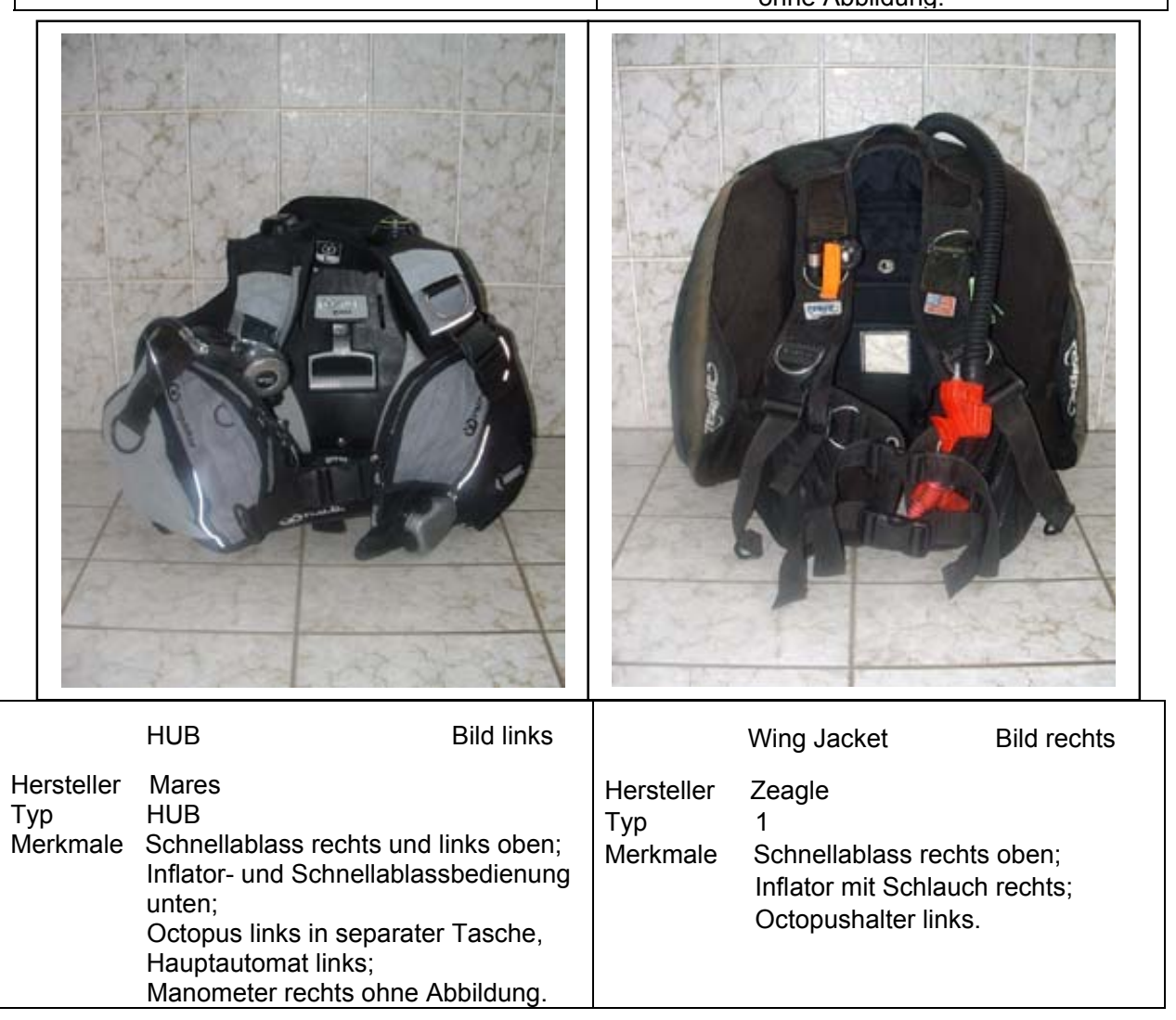

Abbildung 9: $\quad$ Die Auftriebskörper aus Messreihe 2. 


\subsubsection{Bestimmung der Strömungswiderstände der Probanden mit verschiedenen Flossen (Messreihe 3)}

In der dritten Messreihe wurde zuerst der passive Widerstand der Probanden mit unterschiedlichen Flossentypen bestimmt. Die Probanden sind mit Badehose bekleidet und tragen jeweils unterschiedliche Flossen. Der Versuchsaufbau ist analog zu Messreihe 2. Abweichend wird er mit Atemluft über einen langen Mitteldruckschlauch (Nargilée) von außerhalb des Wassers versorgt.

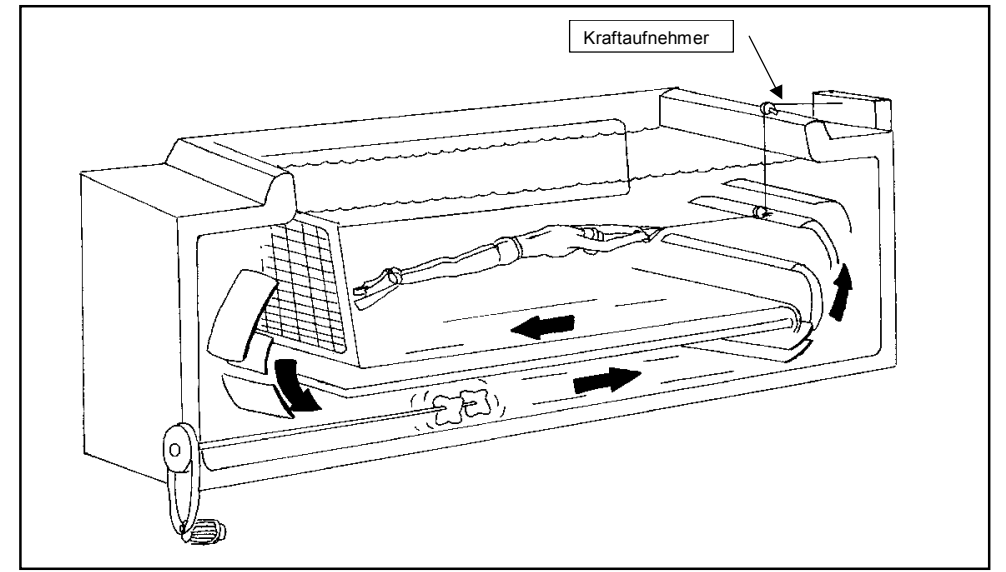

Abbildung 10: Versuchsaufbau für Messreihe 3.

Folgende Flossen kommen zu Einsatz, wobei die Monoflosse nur von Proband B getaucht wird.

1. Monoflosse Hersteller unbekannt:

Länge $71,5 \mathrm{~cm}$, Blatt-Länge $50 \mathrm{~cm}$, Breite $66 \mathrm{~cm}$, Gewicht $1420 \mathrm{~g}$ (Monoflosse)

2. Flossen Hersteller Seemann Sub, Typ SF 2, Größe 41/43:

Länge $56 \mathrm{~cm}$, Blatt-Länge $30 \mathrm{~cm}$, Breite $18 \mathrm{~cm}$ Gewicht $2 \cdot 520 \mathrm{~g}$ (Seemann Sub SF2)

3. Apnoeflossen Hersteller Sporasub, Typ Dessault long 40/42:

Länge $84 \mathrm{~cm}$, Blatt-Länge 56,5 cm, Breite 22,4 cm Gewicht $2 \cdot 960 \mathrm{~g}$ (Sporasub Dessault)

4. Fersenbandflossen Hersteller Mares, Typ Volo, Größe R mit Füßlingen ohne Füßling: Länge $59 \mathrm{~cm}$, Blatt-Länge. $34 \mathrm{~cm}$, Breite $21 \mathrm{~cm}$, Gewicht $2 \cdot 930 \mathrm{~g}$, mit Füßling: Länge $64 \mathrm{~cm}$, Blatt-Länge $34 \mathrm{~cm}$, Breite $21 \mathrm{~cm}$, Gewicht 2 $1250 \mathrm{~g}$, (Mares Volo)

Tabelle 2: $\quad$ Charakteristika der in Messreihe 3 und 4 getragenen Flossen. 


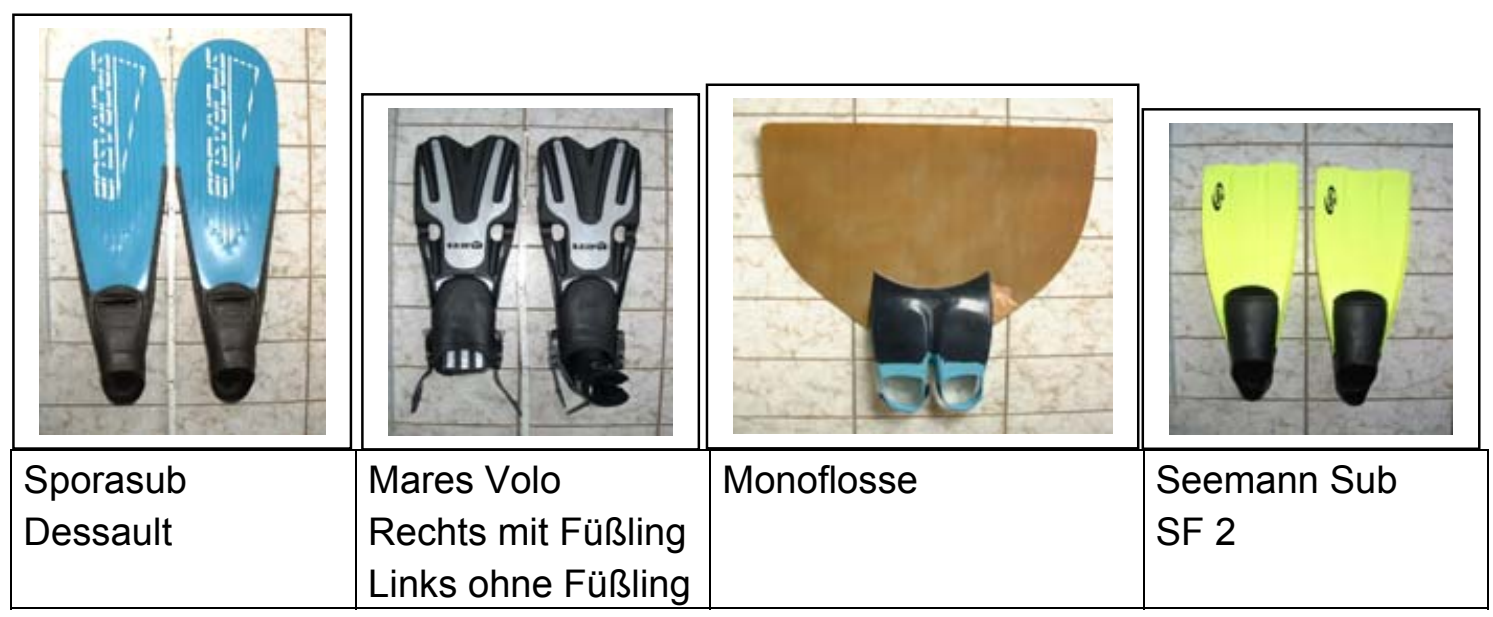

Abbildung 11: Die in Testreihe 3 und 4 untersuchten vier Flossenmodelle. Die Darstellung berücksichtigt annähernd das Größenverhältnis.

Die ausgewählten Flossentypen kommen aus unterschiedlichen Anwendungsgebieten: Die Monoflosse ist eine Hochgeschwindigkeitsflosse. Mit ihr werden die höchstmöglichen Geschwindigkeiten im Tauchen erzielt. Die Flosse wird zum Streckentauchen in Apnoe bis $50 \mathrm{~m}$ Distanz und in allen Streckentauchdisziplinen mit Luftversorgung des Sportlers eingesetzt. Die Flosse Sporasub Dessault ist eine Apnoeflosse, mit welcher der Sportler versucht, unter angehaltenem Atem die zurückgelegte Strecke zu maximieren. Dieses Maximum kann sowohl vertikal (AIDA Kategorie: konstant weight) als auch horizontal (VDST Disziplin Streckentauchen) erbracht werden. Die Flosse Seemann Sub SF 2 ist eine Allround-Schnorchelflosse, die sowohl von reinen Freizeitschnorchlern als auch von kontinuierlich trainierenden Breitensportlern, aber auch von Unterwasserrugbyspielern geschwommen wird. Die Flosse Mares Volo ist eine Geräteflosse, welche mit Füßling geschwommen wird. Geräteflossen verfügen über einstellbares Fersenband. Sie kann nur mit Füßling oder im Trockentauchanzug, keinesfalls jedoch barfuß, getaucht werden.

Die ermittelten Mittelwerte der Widerstände je Strömungsgeschwindigkeit werden in polynomischen Funktionen 2. Grades dargestellt und je Proband und je Flosse verglichen.

\subsubsection{Spiroergometrische Untersuchung mit den Flossen aus der vorgenannten Messreihe (Messreihe 4)}

Bei der spiroergometrischen Untersuchung tragen die Probanden die gleiche Ausrüstung wie in Messreihe 3.

Dabei werden die Probanden nach der Methode der Zusatzkraftbeaufschlagung bei konstanter Geschwindigkeit belastet. Die beiden Probanden tauchen mit $5 \mathrm{~N}$ entlastet, mit $0 \mathrm{~N}$ und $5 \mathrm{~N}$ und $10 \mathrm{~N}$ Belastung wie in der folgenden Tabelle dargestellt: 


\begin{tabular}{|c|c|c|c|c|}
\hline $\begin{array}{c}\text { Zeit- } \\
\text { dauer }\end{array}$ & Zeit & Stufe & Belastung / Entlastung & Zielstellung \\
\hline $3^{\prime}$ & $3^{\prime}$ & & Passiv an der Schleppvorrichtung & Einschwingmessung \\
\hline $3^{\prime}$ & $6^{\prime}$ & 1 & Aktiv bei $-5 \mathrm{~N}$ & $\dot{V} \mathrm{O}_{2}, \dot{V} \mathrm{CO}_{2}$ \\
\hline $1^{\prime}$ & $7^{\prime}$ & & Passiv an der Schleppvorrichtung & \\
\hline $3^{\prime}$ & $10^{\prime}$ & 2 & Aktiv bei 0 N & $\dot{V} \mathrm{O}_{2}, \dot{V} \mathrm{CO}_{2}$ \\
\hline $1^{\prime}$ & $11^{\prime}$ & & Passiv an der Schleppvorrichtung & \\
\hline $3^{\prime}$ & $14^{\prime}$ & 3 & Aktiv bei +5 N & $\dot{V} \mathrm{O}_{2}, \dot{V} \mathrm{CO}_{2}$ \\
\hline $1^{\prime}$ & $15^{\prime}$ & & Passiv an der Schleppvorrichtung & \\
\hline $3^{\prime}$ & $18^{\prime}$ & 4 & $\dot{V} \mathrm{O}_{2}, \dot{V} \mathrm{CO}_{2}$ \\
\hline $3^{\prime}$ & $21^{\prime}$ & & Aktiv bei +10 N & \\
\hline
\end{tabular}

Tabelle 3: $\quad$ Versuchsablauf Messreihe 4.

Als Geschwindigkeit wird entsprechend dem geschätzten Leistungsvermögen der Probanden $0,8 \mathrm{~m} \cdot \mathrm{s}^{-1}$ für alle Flossen mit Ausnahme der Monoflosse ausgewählt. Für die Monoflosse wird auf Grund der höheren Geschwindigkeit, die man mit ihr erzielen kann, $1,0 \mathrm{~m} \cdot \mathrm{s}^{-1}$ festgelegt.

Das Atemgas wird über das umgebungsdruckgesteuerte Ausatemventil zur Analyse abgeleitet. Die spiroergometrische Untersuchung wird mit einem Jäger EOS-Sprint Spirometer mit dynamischer Mischkammer durchgeführt, in der die Exspirationsluft auf $\mathrm{O}_{2}$ - und $\mathrm{CO}_{2}$-Konzentration analysiert wird.

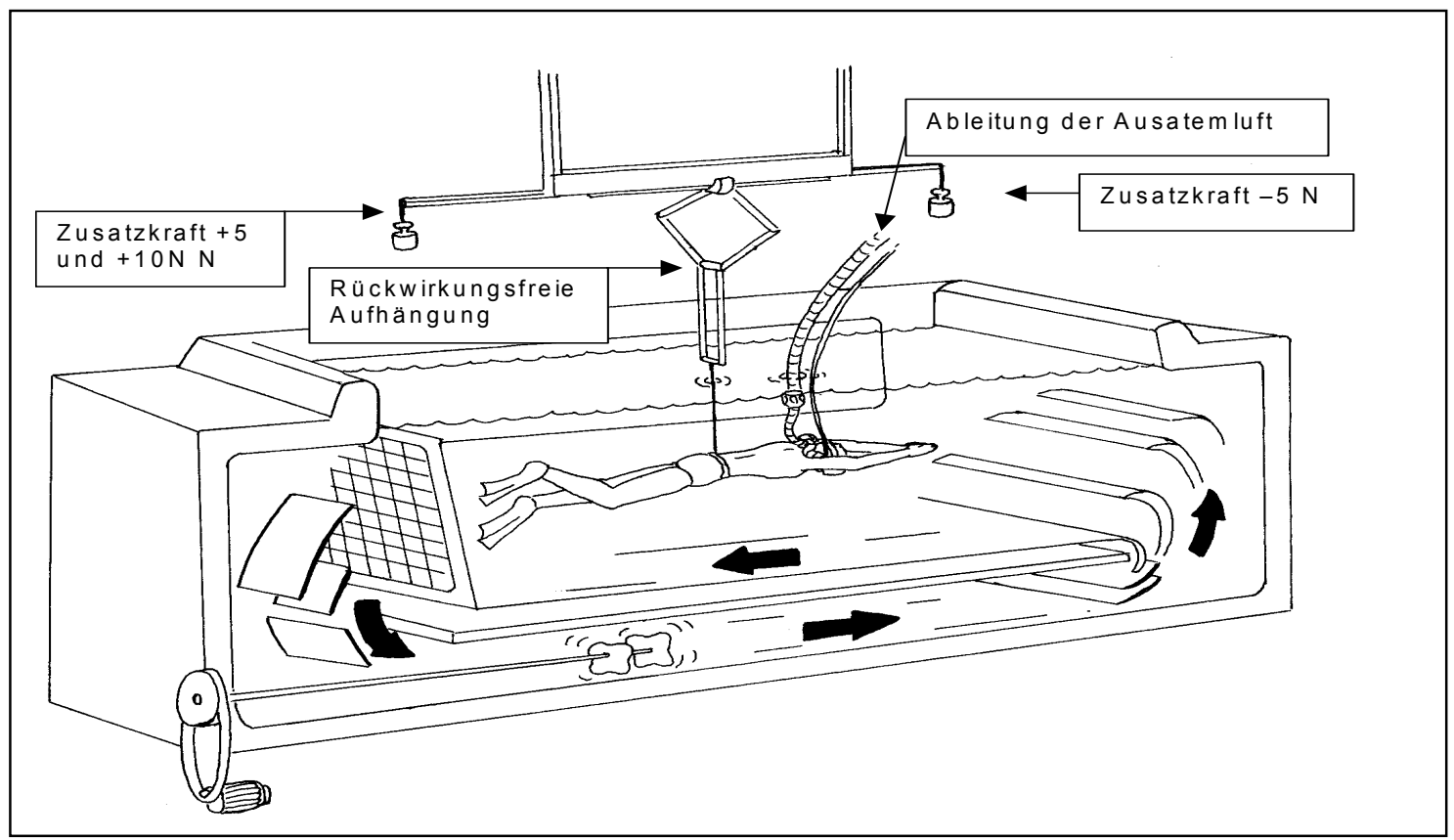

Abbildung 12: Versuchsaufbau für Messreihe 3. 
Jede Belastungsstufe wird mit 3 Minuten festgelegt. Damit wird das untere Limit gewählt, welches in der Literatur als ausreichend benannt ist, um der Problematik des Einschwingverhaltens der Sauerstoffaufnahme und dem Erreichen der Ergostase gerecht zu werden (NIKLAS 1989, 20ff). Nach einem Einschwingverhalten werden näherungsweise konstante Messwerte erwartet, die dann Basis für weitere Berechnungen sind.

Weiterhin wird berücksichtigt, dass in einer Tiefe von ca. 0,5 m getaucht wird, also die Messwerte mit dem Umgebungsdruck $105 \mathrm{kPa}$ (1,05 bar) korrigiert werden müssen.

Dazu werden die erhobenen Daten in einem Diagramm durch eine Gerade verbunden. Das Bestimmtheitsmaß dieser Geraden gibt Auskunft über die Exaktheit der Messpunkte in Beziehung zu der Geraden. Hierbei gilt, dass je größer die Abweichung der Messpunkte vom Mittelwert, desto geringer das Bestimmtheitsmaß. Zusätzlich werden die Standardabweichung und das Konfidenzintervall zur Beurteilung herangezogen.

\subsection{Statistische Methoden und Auswahl der Messwerte}

Die Berechnung der Widerstandskenndaten in Messreihe 2 und 3 erfolgt in einem eigens entwickelten Programm zur Bearbeitung der ermittelten Kraftwerte auf der Basis von Excel 6.0.

Es werden in Messreihe 2 auf jeder Geschwindigkeitsstufe über eine Dauer von drei Minuten ca. 18.000 Messwerte erhoben. Im ersten Schritt werden zur Absicherung der Messreihen alle offensichtlich sinnlosen Werte (negative Kraftwerte) und alle Werte außerhalb der 4-fachen negativen und positiven Standardabweichung ausgeschlossen.

Die danach errechneten Mittelwerte werden hinsichtlich ihrer Datenbasis mit statistischen Kenndaten überprüft, insbesondere der Vergleich des Medians mit dem Mittelwert, die Standardabweichung und die Normalverteilung der Daten, ebenso wie die links oder rechtsseitige Verteilung und die Kurtosis.

In Messreihe 3 wird in gleicher Weise vorgegangen.

In Messreihe 4 ist das Einschwingverhalten der Messwerte anhand der erhobenen Daten zu überprüfen. Durch das Einschwingverhalten in der ersten Phase einer Belastungsstufe werden berücksichtigungsfähige Werte erst im letzten Teil jeder Belastungsstufe erreicht. Im ersten Arbeitsschritt ist festzustellen, wann das Einschwingverhalten abgeschlossen ist. Bei einer Belastungsdauer von 3 Minuten sollten idealerweise die letzten 5 Werte jeder Belastungsstufe in Frage kommen. Sollten über einen längeren Zeitraum stabile Werte abzulesen sein, wird das Zeitintervall entsprechend erweitert. Bei der Auswahl ist jeder einzelne Datensatz zu analysieren, um festzustellen, wann das Einschwingverhalten abgeschlossen ist. 


\subsection{Probandensicherheit}

Am Schwimmkanal wird jeder Proband durch zwei Personen gesichert. Die eine überwacht die Luftzufuhr und die korrekte Schlauchführung, da durch die Strömung der Druck auf die Einatem- und Ausatemschläuche zum Verdrehen der Schläuche und evtl. deren Abgleiten von den Verbindungen führen kann. Darüber hinaus kontrolliert sie optisch die reguläre Atmung und dadurch die Funktion des Atemreglers. Die andere Person hakt den Probanden in eine in allen Ebenen frei bewegliche kardanische Ankoppelungsvorrichtung ein (vgl. NIKLAS et al. 1982). Diese Hilfsperson sichert im Gefahrenfalle den Probanden, da dieser, wenn er eingehakt ist, den Kopf nicht über Wasser halten kann. Bei Störungen muss die Hilfsperson sofort ins Wasser, da der Proband nur mit deren Hilfe ausgehakt werden kann.

Für Notfälle wird die Gabe von $\mathrm{O}_{2}$ normobar über ein Demand-Ventil, mit parallelem Konstant-Flow-Anschluss mit Rebreather-Maske wurde über eine DAN-Oxygen-Unit von Divers-Alert-Network gewährleistet. Der Untersucher ist DAN-Oxygen-Instructor und ausgebildeter Tauchlehrer mit der Ausbilderqualifikation Rettungstauchen.

Die Presslufttauchflaschen sind handelsüblich und entsprechend der Druckbehälterverordnung geprüft. Die Jackets wurden vorab auf ihre ordnungsgemäße Funktion geprüft. Die eingesetzten Lungenautomaten sind von der Fa. tauch-technikservice Göttingen dem jährlichen regulären Service unterzogen worden. 
Zur Analyse der Ausatemluft ist es zwingend notwendig, diese abzuleiten. Die abgeleitete Luft kann dann entweder zur Auswertung aufgefangen werden (DouglasSack) oder der Luftstrom durch Sensoren analysiert werden. Bei der Spiroergometrie auf dem Laufband oder auf dem Fahrradergometer bewegt sich der Proband auf einem stationären Trainingsgerät. Die Analysegeräte können fest neben ihm installiert werden.

Beim Bewegen im Wasser müssen die Analysegerätschaften oder Douglas-Säcke mitgeführt werden. BAUER (1991) beschreibt ein solches Verfahren für das Kanufahren. DiPRAMPERO et al. (1974); PENDERGAST et al. (1977,1996, 2003a, 2003b) und ZAMPARO et al (2002) benutzten eine Plattform, die sich vor dem Schwimmer in einem Kreisbecken mit $60 \mathrm{~m}$ Umfang herbewegte. Die Ableitung der Ausatemluft in den schwimmtaucherischen Tests wird von innen nicht erläutert. Bei Versuchen in der Deutschen Sporthochschule in Köln besteht die Möglichkeit, dass dem Probanden in einer Gondel die Analysegeräte folgen. Die Gondel schwebt über der Wasserlinie. Bei speziellen Fragestellungen kann auf Grund des Aufbaus der Ausrüstung auf eine Ableitung verzichtet werden und die Analyse im Gerät stattfinden. Dieses kann nur bei Kreislaufgeräten durchgeführt werden DRÄGER (1999).

Ein besonderes Augenmerk gilt dabei der Dichtigkeit der Ableitungsgeräte, die im Bereich der Wasserlinie, bzw. zeitweise unter ihr, zum Einsatz kommen. Bei jeder Spiroergometrie an Land spielt die Dichtigkeit der Geräte eine Rolle dahingehend, dass gewährleistet sein muss, dass ausschließlich die zu analysierende Luft aufgefangen wird. Durchmischungen von Kleinstvolumen mit Umgebungsluft als Folge einer minimalen Leckage sind nicht beabsichtigt oder erwünscht. Sie spielen eine untergeordnete Rolle in der Analyse, da die Probleme der Durchmischung und damit von analytischen Fehlern auf Grund normobarer Verhältnisse gering sind.

Im Wasser führt eine solche, auch minimale Undichtigkeit zum Ende des Versuchs, da das System sich mit Wasser füllt. Dadurch können z.B. Sensoren nicht mehr arbeiten, der Ausatemdruck kann sich unphysiologisch erhöhen und der Proband ist gezwungen, den Versuch sofort abzubrechen, da durch das eingedrungene Wasser die Gefahr des Ertrinkens droht. Geschieht dieses beim Tauchen, besteht die Gefahr eines Lungenüberdruckunfalls während des Aufstiegs, wenn der Proband panikartig zur Wasseroberfläche schießt.

Beim Tauchen wird Luft unter Umgebungsdruck geamtet und die Ausatemluft über ein Rückschlagventil in das umgebende Wasser abgegeben. Bei der Spiroergometrie unter Wasser muss der Ausatemstrom zur Analyse an die Wasseroberfläche geleitet werden. Die Luft wird jedoch unter Umgebungsbedingungen unter Wasser ein- und ausgeatmet. Der Umgebungsdruck ist auf Grund der höheren Dichte des Wassers gegenüber der Wasseroberfläche erhöht. 
Druck ist definiert als

$$
P=\frac{F}{A}
$$

Über Wasser wird F durch die Luftsäule über der Erdoberfläche gebildet. Unter Wasser kommt zu dieser Luftsäule die Gewichtskraft der Wassersäule hinzu.

Die Dichte von Süßwasser wird mit

$$
\rho=1,00 \frac{\mathrm{kg}}{\mathrm{dm}^{3}}
$$

angegeben. Das bedeutet, dass pro 1 Meter Wassersäule der Druck um $10 \mathrm{kPa}(0,1$ bar) steigt.

Ein Atemregler liefert die Luft immer unter Umgebungsdruck zuzüglich einem konstruktiv bedingten Einatemwiderstand von 0,5 bis ca. $2,5 \mathrm{kPa}$ ( 5 bis ca. $25 \mathrm{mbar}$ ), variierend je nach Atemregler. Auf Grund der baulichen Bedingungen in einem Atemregler würde es bei widerstandsloser Ableitung der Ausatemluft zu einem Abblasen des Atemreglers kommen. An der Steuermembrane der 2. Stufe würde auf der Wasserseite der Umgebungsdruck des umgebenden Wassers anliegen und auf der Luftseite der Umgebungsdruck der Wasseroberfläche. Durch diese Druckdifferenz kommt es zum Abblasen des Lungenautomaten. Eine Analyse der Ausatemluft ist nicht mehr möglich, da sich Ausatemluft und Einatemluft mischen.

NIKLAS et al. (1988) haben in ihrer Erfindung einen Manostaten angedacht, der den der Tauchtiefe entsprechenden Gegendruck aufbaut. Gesteuert werden soll der Manostat, über ein am Tauchgerät befindliches Manometer, das den Umgebungsdruck an den Manostaten ableitet.

Ein solches System ist von den Autoren jedoch nicht konstruiert worden. Statt dessen benutzten sie ein federbelastetes Ventil, welches im Ausatemschlauch angebracht war. Dieses Ventil wirkte gegen den Ausatemstrom und wurde über ein Handrad manuell gespannt. In der Praxis wurde das Ventil immer gerade so eingestellt, dass ein Abblasen der Luft verhindert wurde. Diese Vorgehensweise kann Auswirkungen auf den Ausatemwiderstand haben, welche nicht zu vernachlässigen sind. So bedeutet schon ein Aufsteigen des Tauchers um $10 \mathrm{~cm}$ eine Erhöhung des Ausatemwiderstands um $10 \mathrm{kPa}$ (100 mbar). Dies bedeutet bei einem voreingestellten Ausatemwiderstand von 0,5 bis ca. $2,5 \mathrm{kPa}$ ( 5 bis ca. 25 mbar) eine Erhöhung, die weit jenseits des zulässigen Grenzwertes von $10 \mathrm{kPa}(25 \mathrm{mbar})$ liegt.

Es erscheint also zwingend notwendig, ein Ventil zu konstruieren, welches die Ausatemluft, gesteuert über den Umgebungsdruck, abgibt.

Erste Überlegungen gingen dorthin, die Druckaufnahme über Sensoren zu gestalten und die Werte an das Gegendruckventil an der Wasseroberfläche zu leiten. Diese Idee wurde verworfen und einer Lösung der Vorzug gegeben, bei der über Membranen der Umgebungsdruck auf Höhe der Ausatmung in den Ausatemstrom eingebracht wird. 
Dadurch ist gewährleistet, dass die Regelung auf Höhe des Umgebungsdrucks stattfindet.

Entwickelt wurde ein umgebungsdruckgesteuertes Exspirationsventil.

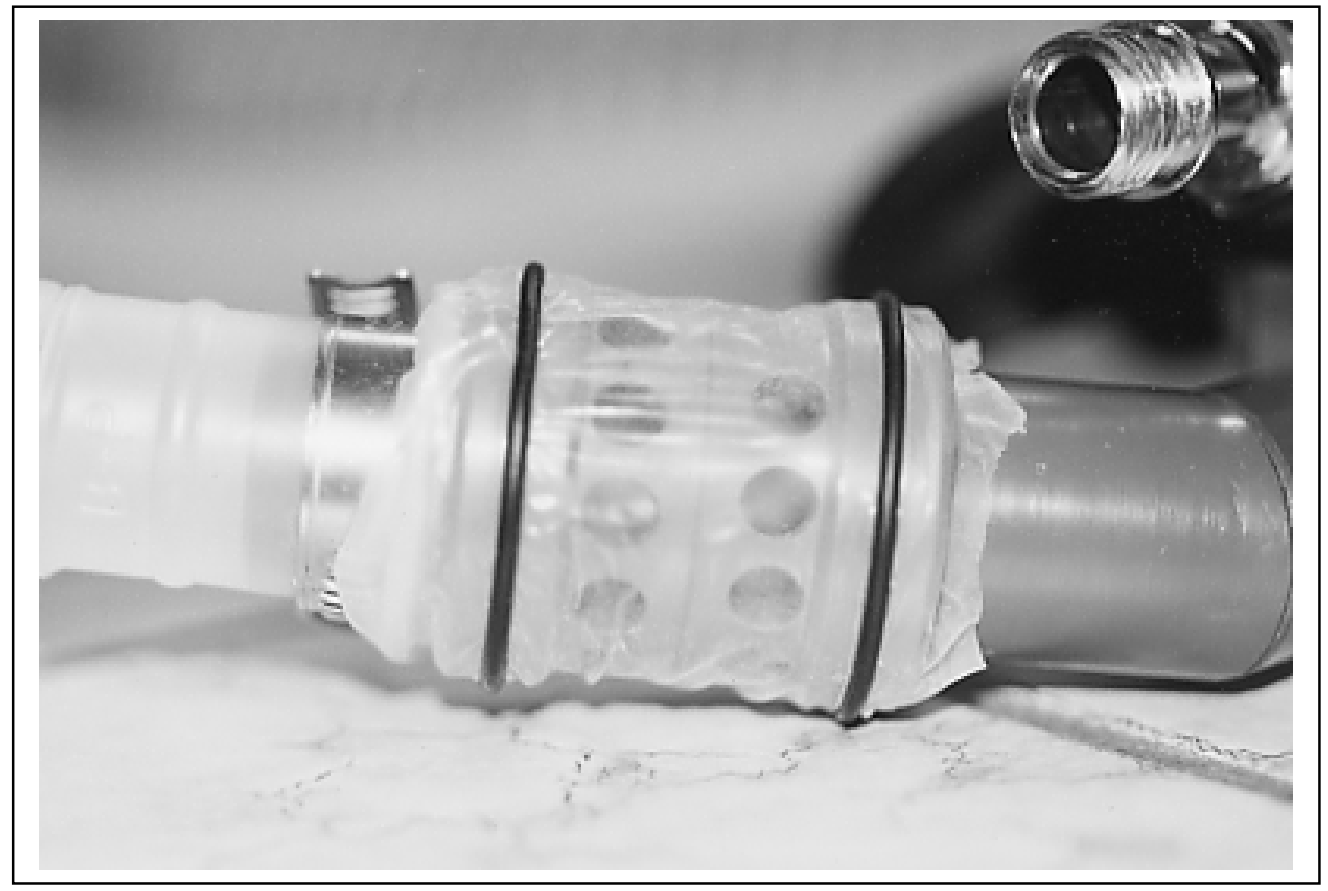

Abbildung 13: Umgebungsdruckgesteuertes Exspirationsventil.

Das Ventil ist mit der Nummer 10005843.4 am 10.2.2000 beim Deutschen Patentund Markenamt angemeldet worden und wurde am 16.8.2001 eingetragen und veröffentlicht. $^{16}$

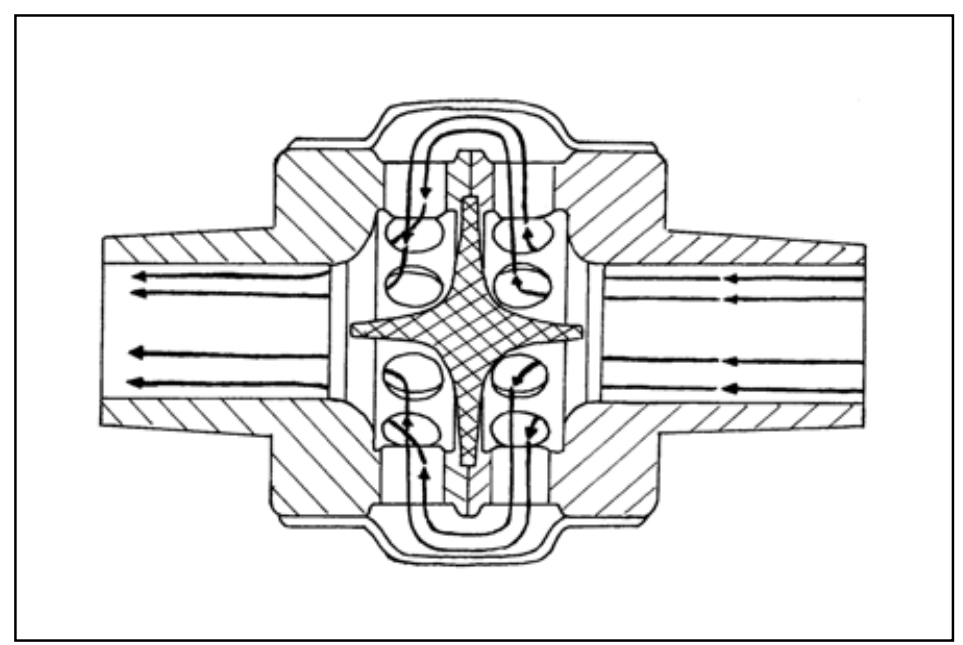

Abbildung 14: Fließdiagramm des umgebungsdruckgesteuerten Exspirationsventils. Strömungsrichtung, wie in Abb. 10 von rechts nach links.

Um die Leistungsfähigkeit des Ventils zu überprüfen, wurde es am 30.8.2003 von der Firma Tauchen + Technik Roland Schmidts in Stadtoldendorf getestet ${ }^{17}$. Die Fa.

${ }^{16}$ Offenlegungsschrift vom 16.8.2001. Siehe 8.1.1. 
Tauchen + Technik Roland Schmidts betreibt mit der Prüfbank Airscan Professional eine durch den öffentlich bestellten und vereidigten Sachverständigen Dr. Dietmar Berndt validierte Prüfbank für Atemregler. In der für die Untersuchung geplanten Tauchtiefe von $1 \mathrm{~m}$ bis zu einer Tiefe von $30 \mathrm{~m}$ lagen die Werte für die Atemarbeit mit maximal 2,33 $\mathrm{J} \cdot \mathrm{I}^{-1}$ (30 m Tiefe) unterhalb des Grenzwertes von 2,5 $\mathrm{J} \cdot \mathrm{I}^{-1}$. Mit einem Einatemdruck von maximal $-1,58 \mathrm{kPa}(-15,8 \mathrm{mbar})$ in $30 \mathrm{~m}$ Tiefe wird der vom Hersteller angegebene Wert von $-2,5 \mathrm{kPa}$ (-25 mbar) Einatemdruck nicht erreicht. Mit $+2,36 \mathrm{kPa}(+23,6 \mathrm{mbar})$ bei $30 \mathrm{~m}$ Wassertiefe wird der Grenzwert der EN 250 für den Ausatemdruck ebenfalls unterschritten, wohingegen hier die Angaben des Herstellers bereits bei $10 \mathrm{~m}$ Wassertiefe knapp überschritten werden ${ }^{18}$. Da andere Ausatemmembranen eingebaut wurden, war eine Erhöhung (Verschlechterung) der Ausatemdrücke zu erwarten. In der geplanten Einsatztiefe bis ca. $1 \mathrm{~m}$ ist der modifizierte Atemregler deutlich innerhalb der Grenzwerte nach EN 250 und innerhalb der Herstellerangaben. Ein Einsatz in Wassertiefen bis zu 10 m wäre denkbar.

\begin{tabular}{|c|c|c|c|}
\hline & Einatemwiderstand & Ausatemwiderstand & Arbeit \\
\hline 0 m Abtauchen & $-0,41 \mathrm{kPa}(-4,1 \mathrm{mbar})$ & $+1,57 \mathrm{kPa}(+15,7 \mathrm{mbar})$ & $1,48 \mathrm{~J} \cdot \mathrm{I}^{-1}$ \\
\hline $1 \mathrm{~m}$ Abtauchen & $-0,58 \mathrm{kPa}(-5,8 \mathrm{mbar})$ & $+1,47 \mathrm{kPa}(+14,7 \mathrm{mbar})$ & $1,36 \mathrm{~J} \cdot \mathrm{I}^{-1}$ \\
\hline 30 m Abtauchen & $-1,58 \mathrm{kPa}(-15,8 \mathrm{mbar})$ & $+2,36 \mathrm{kPa}(+23,6 \mathrm{mbar})$ & $2,33 \mathrm{~J} \cdot \mathrm{I}^{-1}$ \\
\hline 10 m Auftauchen & $-0,88 \mathrm{kPa}(-8,8 \mathrm{mbar})$ & $+1,82 \mathrm{kPa}(+18,2 \mathrm{mbar})$ & $1,77 \mathrm{~J} \cdot \mathrm{I}^{-1}$ \\
\hline $1 \mathrm{~m}$ Auftauchen & $-0,39 \mathrm{kPa}(-3,9 \mathrm{mbar})$ & $+1,48 \mathrm{kPa}(+14,8 \mathrm{mbar})$ & $1,40 \mathrm{~J} \cdot \mathrm{I}^{-1}$ \\
\hline $0 \mathrm{~m}$ Auftauchen & - 0,43 kPa (-4,3 mbar) & $+1,46 \mathrm{kPa}(+14,6 \mathrm{mbar})$ & $1,41 \mathrm{~J} \cdot \mathrm{I}^{-1}$ \\
\hline $\begin{array}{l}\text { Grenzwert nach } \\
\text { EN } 250\end{array}$ & $-2,50 \mathrm{kPa}(-25,0 \mathrm{mbar})$ & $+2,50 \mathrm{kPa}(+25,0 \mathrm{mbar})$ & $3,00 \mathrm{~J} \cdot \mathrm{I}^{-1}$ \\
\hline $\begin{array}{l}\text { Herstellerangaben } \\
\text { Fa. Balzer }\end{array}$ & $-2,50 \mathrm{kPa}(-25,0 \mathrm{mbar})$ & $+1,80 \mathrm{kPa}(+18,0 \mathrm{mbar})$ & $\begin{array}{c}\text { Keine } \\
\text { Angaben }\end{array}$ \\
\hline
\end{tabular}

Tabelle 4: Kenndaten des umgebungsdruckgesteuerten Exspirationsventils. Neben den SI-Einheiten sind die Daten der Prüfprotokolle der Fa Schmidts und des Schreibens der Fa. Balzer übernommen worden.

Da der Atemregler die Einatemluft unter Umgebungsdruck liefert, entsteht ein sich proportional zum steigenden Umgebungsdruck ändernder, erhöhter Volumenstrom. Dieser ist über das Gesetz von Boyle/Mariotte zurückzurechnen:

$$
p \cdot v=\text { konstant für } T=\text { konstant }
$$

Wegen der Bedingungen im Schwimmkanal kann auf die Korrektur hinsichtlich realer Gase in diesem Fall verzichtet werden, da:

\footnotetext{
${ }^{17}$ Prüfdiagramme der Fa. Tauchen + Technik Roland Schmidts, Stadtoldendorf. Mit freundlicher Genehmigung. Siehe 8.1.2 .

${ }^{18}$ Daten des Sherwood Brut übermittel am 22.8.2004. Mit freundlicher Genehmigung der Fa.

Balzer, Spessartstr. 13, D-36341 Lauterbach. Siehe 8.1.3.
} 
- die Druckdifferenz $\Delta \mathrm{p}$ im Schwimmkanal klein ist,

- systematische Fehler durch Differenzbildung nahezu beseitigt werden,

- der analytische Faktor auf Grund des relativ geringen Wirkungsgrades groß ist.

Auf die Sauerstoffaufnahme bleiben die veränderten Druckverhältnisse praktisch ohne Einfluss, da sich die Partialdrücke, entsprechend dem Gasgesetz von Dalton, im Exspirationsstrom proportional zum Gesamtvolumen verhalten.

$$
p_{\text {ges }}=p_{1}+p_{2}+p_{3}+\ldots p_{n}
$$




\subsection{Die realen Strömungsbedingungen im Strömungskanal (Messreihe 1) und die Kalibrierung des Kraftaufnehmers}

Die Ermittlung der realen Strömungsverhältnisse im Strömungskanal wird mit Messflügeln vorgenommen. Die 5 Messflügel (Mfl) sind jeweils in den Tiefen $80 \mathrm{~mm}$ und $470 \mathrm{~mm}$ sowohl vorne im Strömungskanal, also vor den Probanden, in der Mitte des Kanals, als auch hinten im Strömungskanal, d.h. hinter den Probanden positioniert. Beispielhaft sind die Messwerte in $470 \mathrm{~mm}$ Tiefe hinten für $0,3 \mathrm{~m} \cdot \mathrm{s}^{-1}$ und in $80 \mathrm{~mm}$ Tiefe vorne für $1,5 \mathrm{~m} \cdot \mathrm{s}^{-1}$ abgebildet.

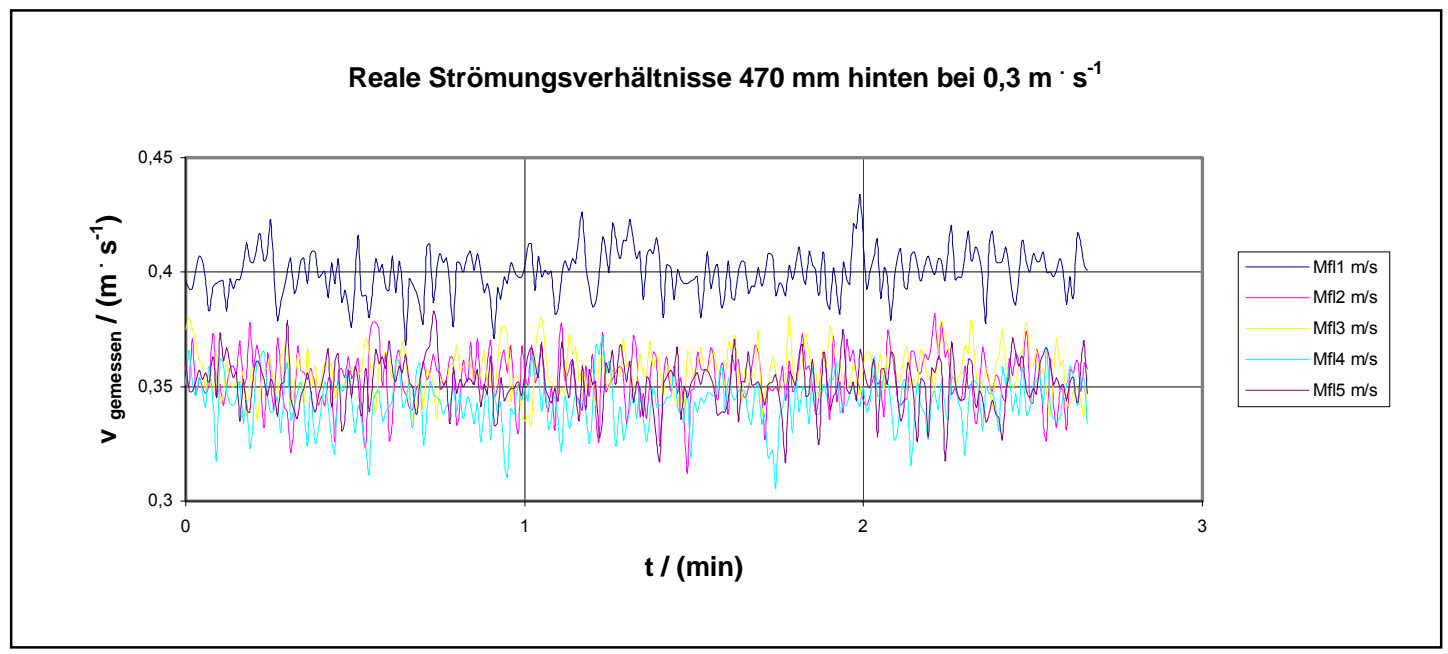

Abbildung 15: Messung der realen Strömungsverhältnisse in $470 \mathrm{~mm}$ Tiefe hinten mit $5 \mathrm{Mfl}$ bei eingestellten $0,3 \mathrm{~m} \cdot \mathrm{s}^{-1}$.

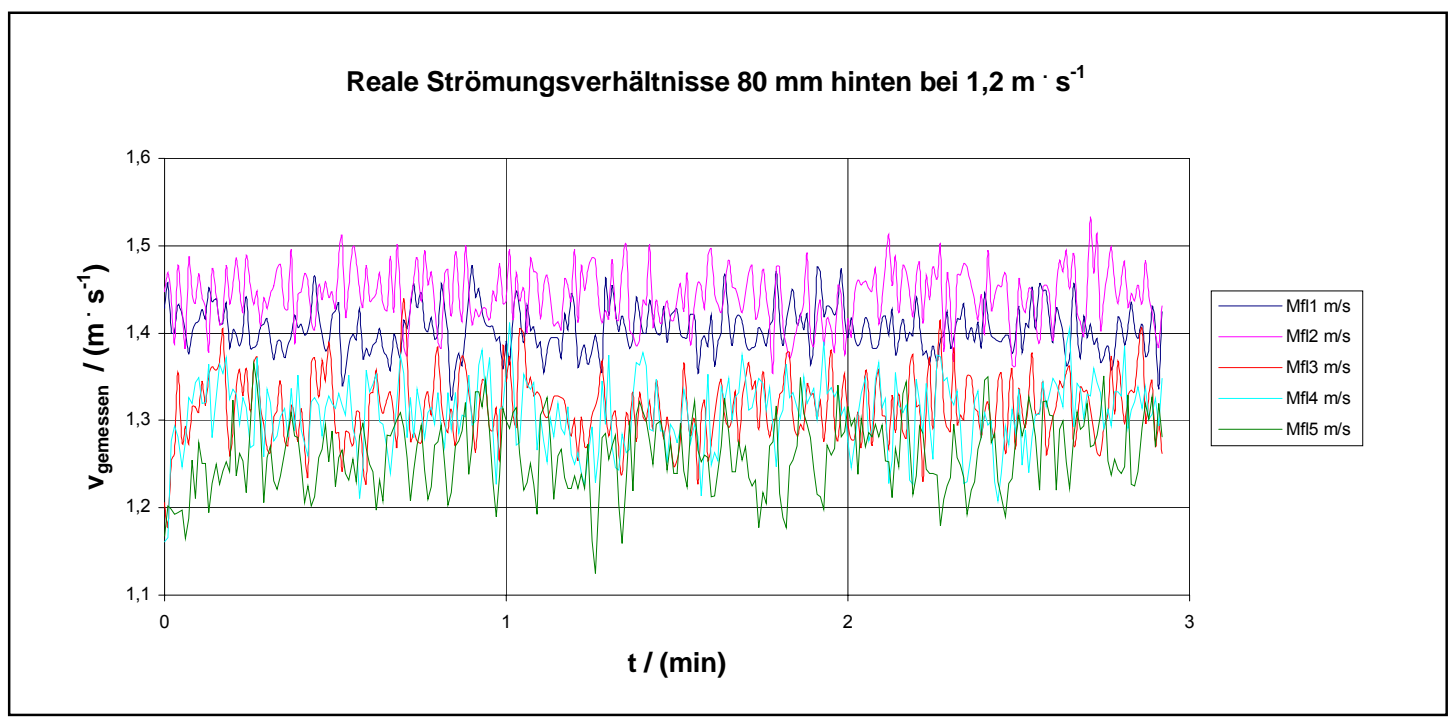

Abbildung 16: Messung der realen Strömungsverhältnisse in $80 \mathrm{~mm}$ Tiefe hinten mit $5 \mathrm{Mfl}$ bei eingestellten $1,2 \mathrm{~m} \cdot \mathrm{s}^{-1}$.

Die an den Messflügeln erhobenen Daten zeigen eine turbulente Strömungssituation. Begründet durch auftretende Turbulenzen sind für die beiden abgebildeten Kurven die 
Maxima $1,533 \mathrm{~m} \cdot \mathrm{s}^{-1}$ bei $1,2 \mathrm{~m} \cdot \mathrm{s}^{-1}$ und $0,434 \mathrm{~m} \cdot \mathrm{s}^{-1}$ bei $0,3 \mathrm{~m} \cdot \mathrm{s}^{-1}$ und die Minima $1,125 \mathrm{~m} \cdot \mathrm{s}^{-1}$ bei $1,2 \mathrm{~m} \cdot \mathrm{s}^{-1}$ und $0,306 \mathrm{~m} \cdot \mathrm{s}^{-1}$ bei $0,3 \mathrm{~m} \cdot \mathrm{s}^{-1}$. Gemittelt ergeben sich reale Strömungsgeschwindigkeiten von $1,346 \mathrm{~m} \cdot \mathrm{s}^{-1}$ bei $1,2 \mathrm{~m} \cdot \mathrm{s}^{-1}$ und $0,3609 \mathrm{~m} \cdot \mathrm{s}^{-1}$ bei $0,3 \mathrm{~m} \cdot \mathrm{s}^{-1}$ für die angegebenen Tiefen.

In den folgenden beiden Diagrammen sind die polynomischen Trendlinien differenziert nach allen fünf Messflügeln und den drei inneren Messflügeln dargestellt. Innerhalb der Diagramme wird differenziert nach der Messtiefe von 80 und $470 \mathrm{~mm}$ und es werden die Werte beider Tiefen zusammengefasst. Unterer und oberer Konfidenzbereich können nicht aufgetragen werden, da mit maximalen Konfidenzwerten von 0,0034 N bei $470 \mathrm{~mm} / 5 \mathrm{Mfl}, 0,0026 \mathrm{~N}$ bei $470 \mathrm{~mm} / 3 \mathrm{Mfl}, 0,0304 \mathrm{~N}$ bei $80 \mathrm{~mm} / 5 \mathrm{Mfl}, 0,0040 \mathrm{~N}$ bei $80 \mathrm{~mm} / 3 \mathrm{Mfl}, 0,0037 \mathrm{~N}$ für beide Tiefen/5Mfl und 0,0049 $\mathrm{N}$ für beide Tiefen/3 Mfl eine graphische Darstellung nicht mehr möglich ist, da die Kurven sich überlagern.

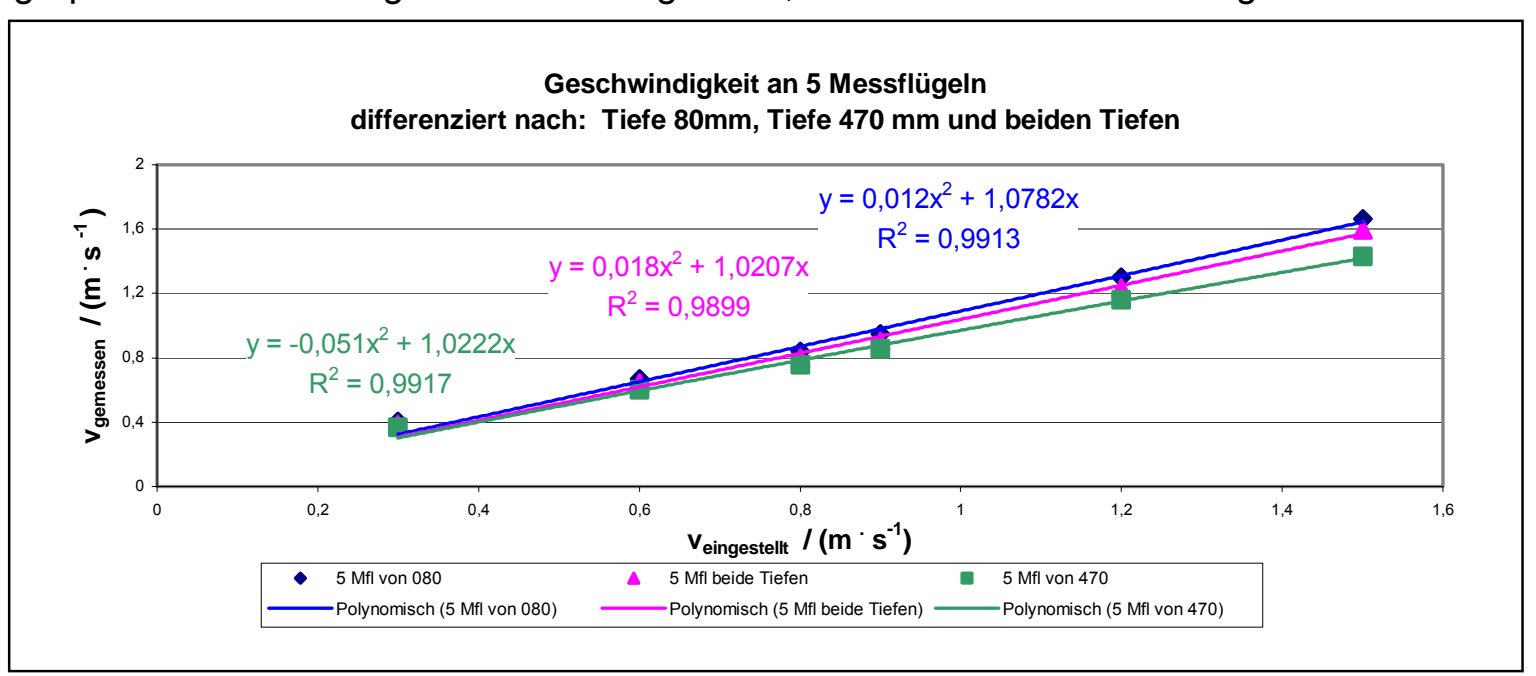

Abbildung 17: Mittelwerte der realen Geschwindigkeit an 5 Messflügeln differenziert nach $80 \mathrm{~mm}$ Tiefe, $470 \mathrm{~mm}$ Tiefe und dem Mittelwert beider Tiefen.

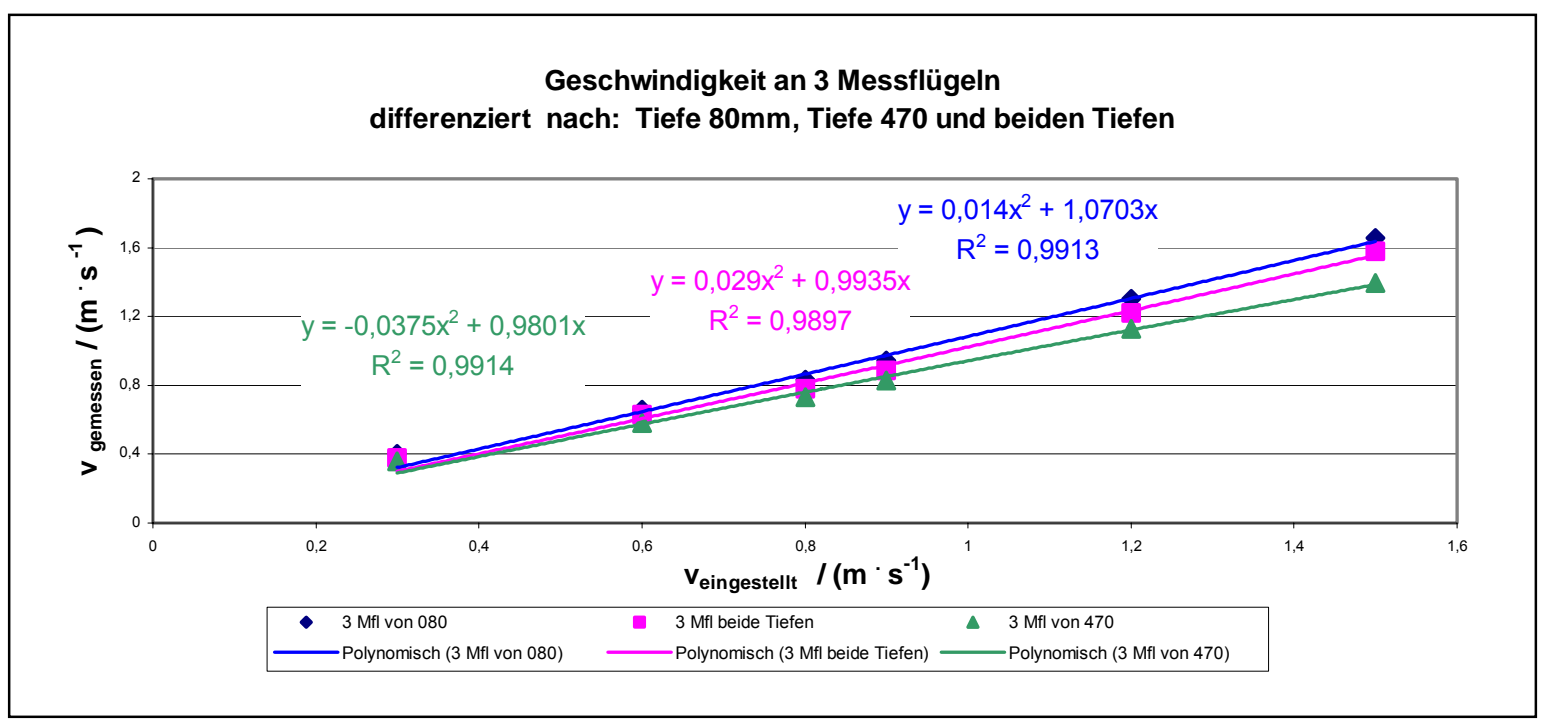

Abbildung 18: Mittelwerte der realen Geschwindigkeit an 3 Messflügeln differenziert nach $80 \mathrm{~mm}$ Tiefe, $470 \mathrm{~mm}$ Tiefe und dem Mittelwert beider Tiefen. 
Die mit den Messflügeln ermittelten Daten ergeben gegenübergestellt eine polynomische Funktion, die sowohl für die Maximalwerte als auch für die Minimalwerte annähernd einer Geraden entspricht. Dadurch zeigt sich, dass die Strömung im Kanal, wie erwartet, turbulent und nicht laminar ist. Die Ergebnisse liegen aber so eng beieinander, dass von konstanten Bedingungen im Kanal ausgegangen werden kann. ${ }^{19}$

Da die Versuche unter Wasser stattfinden, ist der für die Untersuchung relevante Bereich durch die Tiefe $470 \mathrm{~mm}$ und die Breite der inneren drei Messflügel abgebildet. Die Daten für die Stufe $1,0 \mathrm{~m} \cdot \mathrm{s}^{-1}$ wurden mit Hilfe der Funktionsformel errechnet. Aus dieser Vorgabe leiten sich die folgenden realen Geschwindigkeiten ab:

\begin{tabular}{|l|l|l|l|}
\hline $\begin{array}{l}\text { Anstromgeschwin- } \\
\text { digkeit Einstellung } \\
\text { am Kanal in } \mathrm{m} \cdot \mathrm{s}^{-1}\end{array}$ & $\begin{array}{l}\text { Umrechnung } \\
\text { in } \mathrm{km} \cdot \mathrm{h}^{-1}\end{array}$ & $\begin{array}{l}\text { Gemessene } \\
\text { Anstromgeschwindigkeit in } \\
\text { der Mitte des Kanals 470mm } \\
\text { tief in } \mathrm{m} \cdot \mathrm{s}^{-1}\end{array}$ & $\begin{array}{l}\text { Umrechnung } \\
\text { in } \mathrm{km} \cdot \mathrm{h}^{-1}\end{array}$ \\
\hline $0,3 \mathrm{~m} \cdot \mathrm{s}^{-1}$ & $1,08 \mathrm{~km} \cdot \mathrm{h}^{-1}$ & $0,35666289 \mathrm{~m} \cdot \mathrm{s}^{-1}$ & $1,284 \mathrm{~km} \cdot \mathrm{h}^{-1}$ \\
\hline $0,6 \mathrm{~m} \cdot \mathrm{s}^{-1}$ & $2,16 \mathrm{~km} \cdot \mathrm{h}^{-1}$ & $0,58172035 \mathrm{~m} \cdot \mathrm{s}^{-1}$ & $2,094 \mathrm{~km} \cdot \mathrm{h}^{-1}$ \\
\hline $0,8 \mathrm{~m} \cdot \mathrm{s}^{-1}$ & $2,88 \mathrm{~km} \cdot \mathrm{h}^{-1}$ & $0,72881606 \mathrm{~m} \cdot \mathrm{s}^{-1}$ & $2,624 \mathrm{~km} \cdot \mathrm{h}^{-1}$ \\
\hline $0,9 \mathrm{~m} \cdot \mathrm{s}^{-1}$ & $3,24 \mathrm{~km} \cdot \mathrm{h}^{-1}$ & $0,8289072 \mathrm{~m} \cdot \mathrm{s}^{-1}$ & $2,988 \mathrm{~km} \cdot \mathrm{h}^{-1}$ \\
\hline $1,0 \mathrm{~m} \cdot \mathrm{s}^{-1}$ & $3,60 \mathrm{~km} \cdot \mathrm{h}^{-1}$ & $0,9426 \mathrm{~m} \cdot \mathrm{s}^{-1}$ & $3,393 \mathrm{~km} \cdot \mathrm{h}^{-1}$ \\
\hline $1,2 \mathrm{~m} \cdot \mathrm{s}^{-1}$ & $4,32 \mathrm{~km} \cdot \mathrm{h}^{-1}$ & $1,12831264 \mathrm{~m} \cdot \mathrm{s}^{-1}$ & $4,062 \mathrm{~km} \cdot \mathrm{h}^{-1}$ \\
\hline $1,5 \mathrm{~m} \cdot \mathrm{s}^{-1}$ & $5,40 \mathrm{~km} \cdot \mathrm{h}^{-1}$ & $1,39516463 \mathrm{~m} \cdot \mathrm{s}^{-1}$ & $5,022 \mathrm{~km} \cdot \mathrm{h}^{-1}$ \\
\hline
\end{tabular}

Tabelle 5: Reale Geschwindigkeiten im Strömungskanal.

Im Folgenden wird die Geschwindigkeit mit den real gemessenen Werten gerundet auf die vierte Nachkommastelle angegeben. Alle Berechnungen basieren auf den gemessen Werten bis zur achten Nachkommastelle, wie in Tabelle 5 aufgeführt.

\footnotetext{
${ }^{19}$ Unter 8.1.4 sind die Mittelwerte, die Standardabweichung und das Konfidenzintervall für jede Geschwindigkeitsstufe auf jeder Tiefe angegeben.

Die Werte der eingestellten Geschwindigkeitsstufen $0,6 \mathrm{~m} \cdot \mathrm{s}^{-1}$ in der Mitte und 1,5 $\mathrm{m} \cdot \mathrm{s}^{-1}$ vorne jeweils in $470 \mathrm{~mm}$ Wassertiefe sind durch Übertragungsfehler nicht vorhanden. Vergleiche dazu beiliegende CD-Rom, Datei: „Bedingungen_der_Strömung_im_Kanal.xls“.
} 
Das folgende Diagramm zeigt den zeitlichen Ablauf des Einschwingverhaltens des Kraftaufnehmers. Aus der Position ohne Last wird nach kurzem, zeitlich eng umrissenen Einschwingverhalten (von Sekunde 4 bis Sekunde 8 ) die Belastungsstufe erreicht.

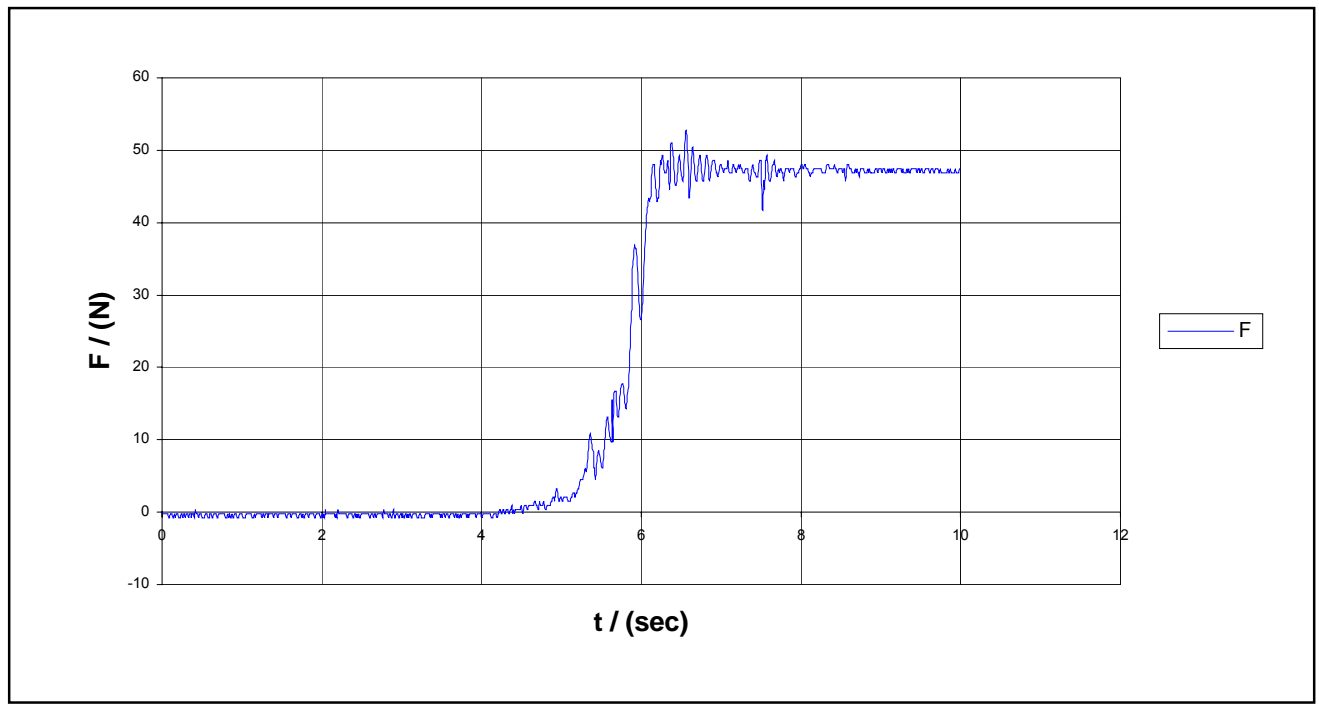

Abbildung 19: Das Einschwingverhalten des Kraftaufnehmers.

Die Linearität der Messdaten wird vorausgesetzt, so dass mit der in der Abbildung abgebildeten Formel

$$
y=-0,4767 x-0,2168
$$

die erhoben Messdaten für die Messreihe 2 exakt in Kraftwerte $F[N]$ umgerechnet werden können.

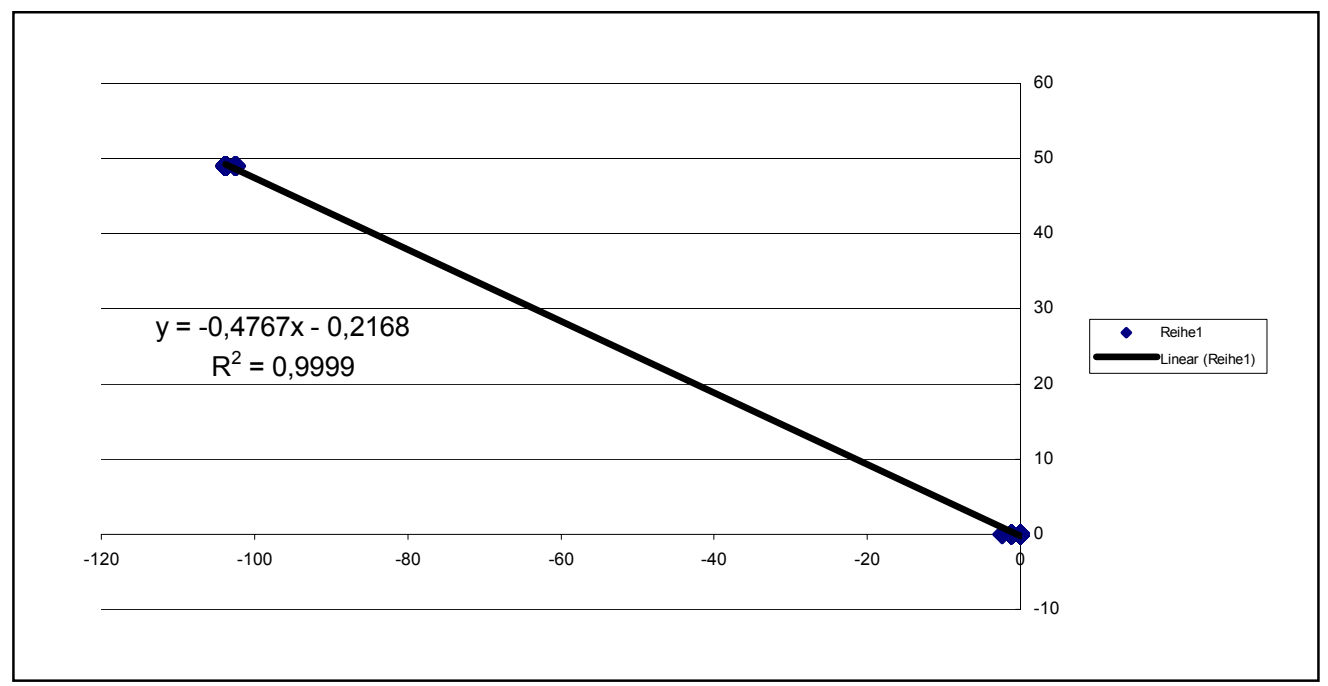

Abbildung 20: Kalibrierung des Kraftaufnehmers. ${ }^{20}$

${ }^{20}$ Messdaten siehe CD-ROM, Datei: „Kalibrierung_des_Kraftaufnehmers.xIs“. 


\subsection{Die Strömungswiderstände $F_{\mathrm{Dp}}$ der Probanden mit unterschiedlichen}

Auftriebskörpern (Messreihe 2)

\subsubsection{Die Kraftwerte für jeden Auftriebskörper}

Für Proband A werden, entsprechend der unter 3.4 beschriebenen Vorgehensweise maximal 1,622 \% der Messwerte einer Versuchsreihe (Wing Jacket/0,3 $\mathrm{m} \cdot \mathrm{s}^{-1}$ ) ausgeschlossen. Bei Proband B werden maximal 1,139 \% der Messwerte einer Versuchsreihe (Jacket/0,3 $\mathrm{m} \cdot \mathrm{s}^{-1}$ ) ausgeschlossen. Die Raten liegen in ihrer Gesamtheit unter dem Grenzwert von 2\% für den Maximalausschluss. Mittelwert und Median $^{21}$ liegen hinreichend exakt beieinander. Die Abweichungen betragen bei Proband A maximal $-0,31 \mathrm{~N}$ und $+0,62 \mathrm{~N}$ und bei Proband $\mathrm{B}-0,59 \mathrm{~N}$ und $+0,70 \mathrm{~N}$. Die Schiefe $^{22}$, als Maß für die Normalverteilung, liegt zwischen $-0,18$ und $+0,62$ für Proband $A$ und zwischen $-0,31$ und $+0,49$ für Proband $B$. Im Folgenden sind die gemessenen Kraftwerte für beide Probanden tabellarisch aufgeführt. Die Kraftwerte steigen mit zunehmender Geschwindigkeit an.

\begin{tabular}{|l|c|c|c|c|c|}
\hline Proband A & $0,3567 /\left(\mathrm{m} \cdot \mathrm{s}^{-1}\right)$ & $0,5817 /\left(\mathrm{m} \cdot \mathrm{s}^{-1}\right)$ & $0,8289 /\left(\mathrm{m} \cdot \mathrm{s}^{-1}\right)$ & $1,1283 /\left(\mathrm{m} \cdot \mathrm{s}^{-1}\right)$ & $1,3951 /\left(\mathrm{m} \cdot \mathrm{s}^{-1}\right)$ \\
\hline & $\mathrm{F}_{\mathrm{Dp}}[\mathrm{N}]$ & $\mathrm{F}_{\mathrm{Dp}}[\mathrm{N}]$ & $\mathrm{F}_{\mathrm{Dp}}[\mathrm{N}]$ & $\mathrm{F}_{\mathrm{Dp}}[\mathrm{N}]$ & $\mathrm{F}_{\mathrm{Dp}}[\mathrm{N}]$ \\
\hline Wing Jacket & 16,72 & 36,86 & 66,95 & 111,78 & 170,73 \\
\hline Kragenweste & 24,24 & 44,59 & 74,40 & 126,96 & 198,50 \\
\hline Jacket & 18,71 & 37,16 & 68,33 & 110,86 & 163,56 \\
\hline HUB & 17,81 & 36,77 & 71,68 & 128,42 & 203,48 \\
\hline
\end{tabular}

Tabelle 6: $\quad$ Kraftwerte für Proband A.

\begin{tabular}{|l|c|c|c|c|c|}
\hline Proband B & $0,3567 /\left(\mathrm{m} \cdot \mathrm{s}^{-1}\right)$ & $0,5817 /\left(\mathrm{m} \cdot \mathrm{s}^{-1}\right)$ & $0,8289 /\left(\mathrm{m} \cdot \mathrm{s}^{-1}\right)$ & $1,1283 /\left(\mathrm{m} \cdot \mathrm{s}^{-1}\right)$ & $1,3951 /\left(\mathrm{m} \cdot \mathrm{s}^{-1}\right)$ \\
\hline & $\mathrm{F}_{\mathrm{Dp}}[\mathrm{N}]$ & $\mathrm{F}_{\mathrm{Dp}}[\mathrm{N}]$ & $\mathrm{F}_{\mathrm{Dp}}[\mathrm{N}]$ & $\mathrm{F}_{\mathrm{Dp}}[\mathrm{N}]$ & $\mathrm{F}_{\mathrm{Dp}}[\mathrm{N}]$ \\
\hline Wing Jacket & 18,24 & 39,55 & 68,51 & 110,07 & 173,12 \\
\hline Kragenweste & 23,03 & 42,19 & 69,67 & 117,50 & 181,45 \\
\hline Jacket & 15,84 & 32,52 & 61,50 & 107,90 & 166,67 \\
\hline HUB & 17,82 & 34,99 & 68,41 & 120,85 & 197,02 \\
\hline
\end{tabular}

Tabelle 7: $\quad$ Kraftwerte für Proband $B$.

Die fünf Messwerte für jeden Auftriebskörper sind polynomisch verbunden und in den nachfolgenden Grafiken jeweils je Proband gegenübergestellt. Die Bestimmtheitsmaße der einzelnen Kurven dokumentieren eine hohe Übereinstimmung mit der polynomischen Funktion 2. Grades.

\begin{tabular}{|l|c|c|}
\hline & $R^{2}$ Proband $A$ & $R^{2}$ Proband $B$ \\
\hline Wing Jacket & 0,9994 & 0,9975 \\
\hline Kragenweste & 0,9972 & 0,9975 \\
\hline Jacket & 0,9997 & 0,9994 \\
\hline HUB & 0,9989 & 0,9976 \\
\hline
\end{tabular}

Tabelle 8: $\quad$ Bestimmtheitsmaß der Kraftkurven jedes Auftriebskörpers.

${ }^{21}$ Die Tabellen für den Vergleich zwischen Median und Mittelwert sind im Anhang 8.1.5 .

22 Die Tabellen für die Schiefe und vier exemplarische Diagramme der Verteilung sind im Anhang 8.1.6 und 8.1.7. Die Messwerte sind auf der CD-ROM in den Dateien: „Kraftwerte_ Auftriebskörper_Proband_A.xls“ und „Kraftwerte_Auftriebskörper_Proband_B.xls“. 
Die Kurven der Kraftwerte stellen sich als Exponentialfunktion dar. Das Wing Jacket wird bei beiden Probanden mit näherungsweise identischen Kraftwerten gemessen. Die Kurvenverläufe sind annähernd identisch.

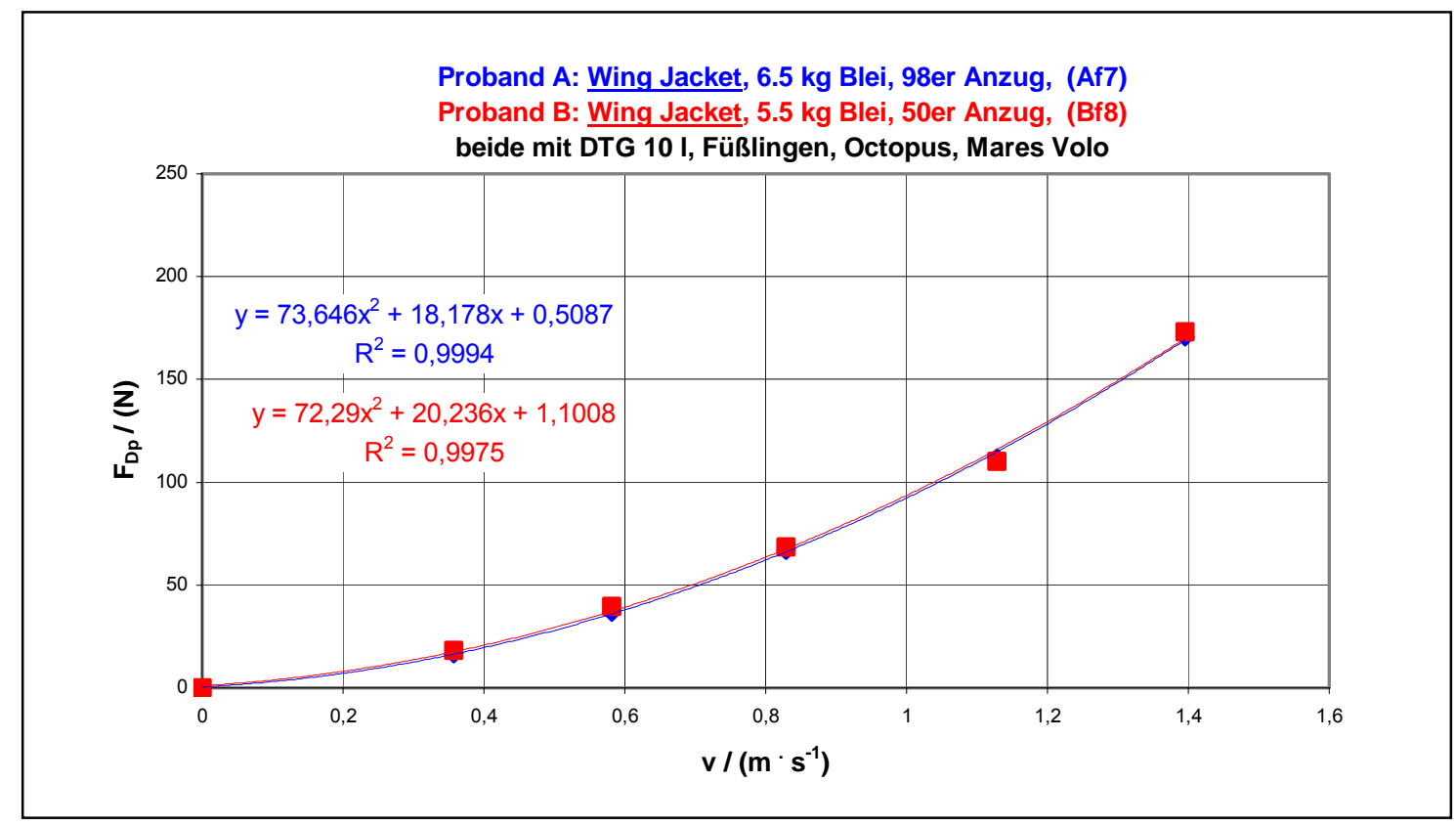

Abbildung 21: Kraftwerte $\mathrm{F}_{\mathrm{Dp}} /(\mathrm{N})$ für beide Probanden mit dem Wing Jacket.

Bei der Kragenweste sind die Kurvenverläufe bis $0,5817 \mathrm{~m} \cdot \mathrm{s}^{-1}$ angenähert identisch. Danach weichen die Daten und somit die Kurven voneinander ab. Die Kurve von Proband A verläuft steiler als die von Proband B. Bei $0,8289 \mathrm{~m} \cdot \mathrm{s}^{-1}$ beträgt die Differenz zwischen beiden Kurven 9,46 N und bei 1,3952 $\mathrm{m} \cdot \mathrm{s}^{-1} 17,05 \mathrm{~N}$.

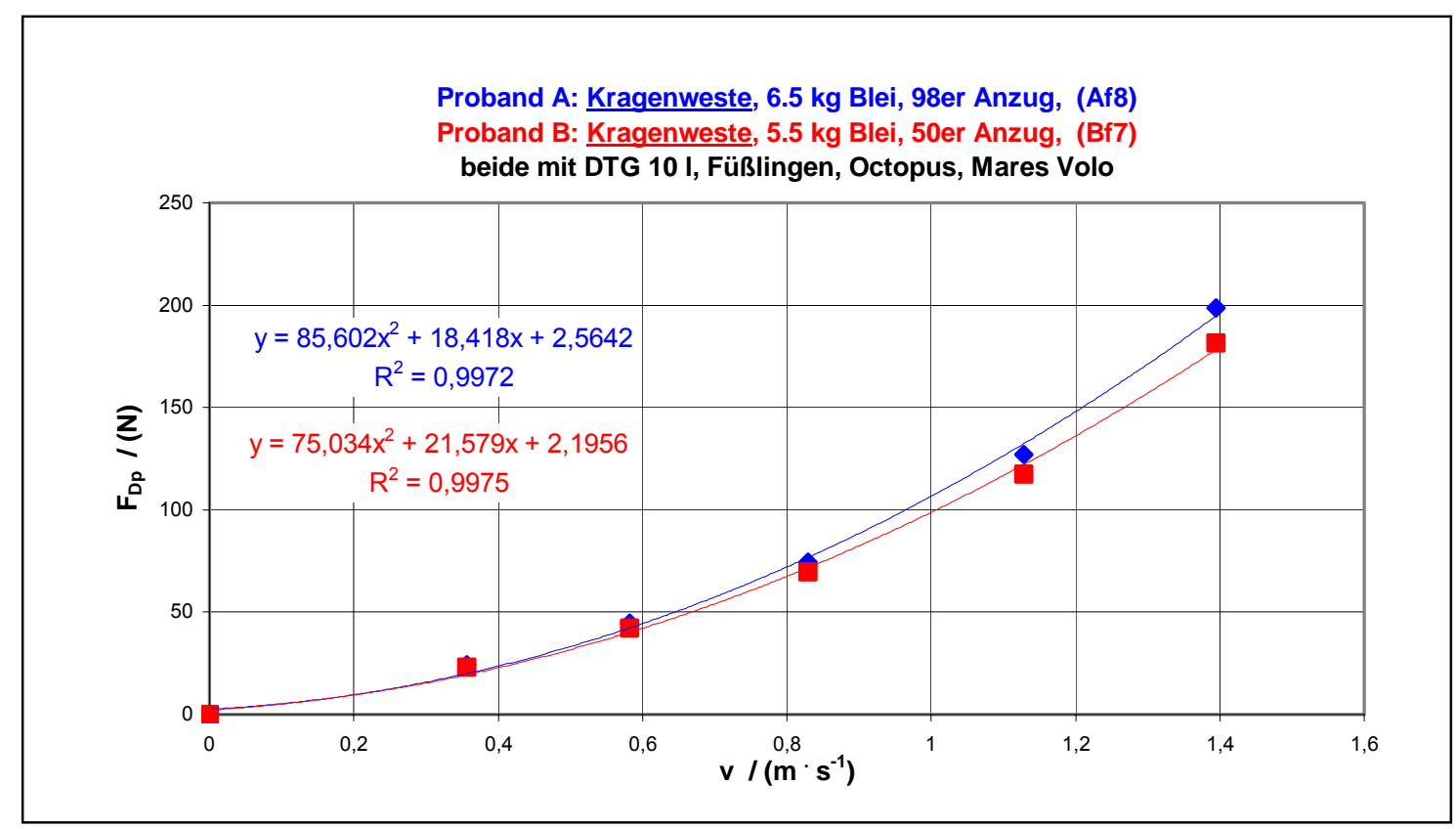

Abbildung 22: Kraftwerte $\mathrm{F}_{\mathrm{Dp}} /(\mathrm{N})$ für beide Probanden mit der Kragenweste. 
Beim HUB sind die Kurven für beide Probanden annähernd identisch. Mit dem HUB erreichen beide Probanden in den letzten beiden Geschwindigkeitsstufen jeweils die Maximalwerte der Messreihe.

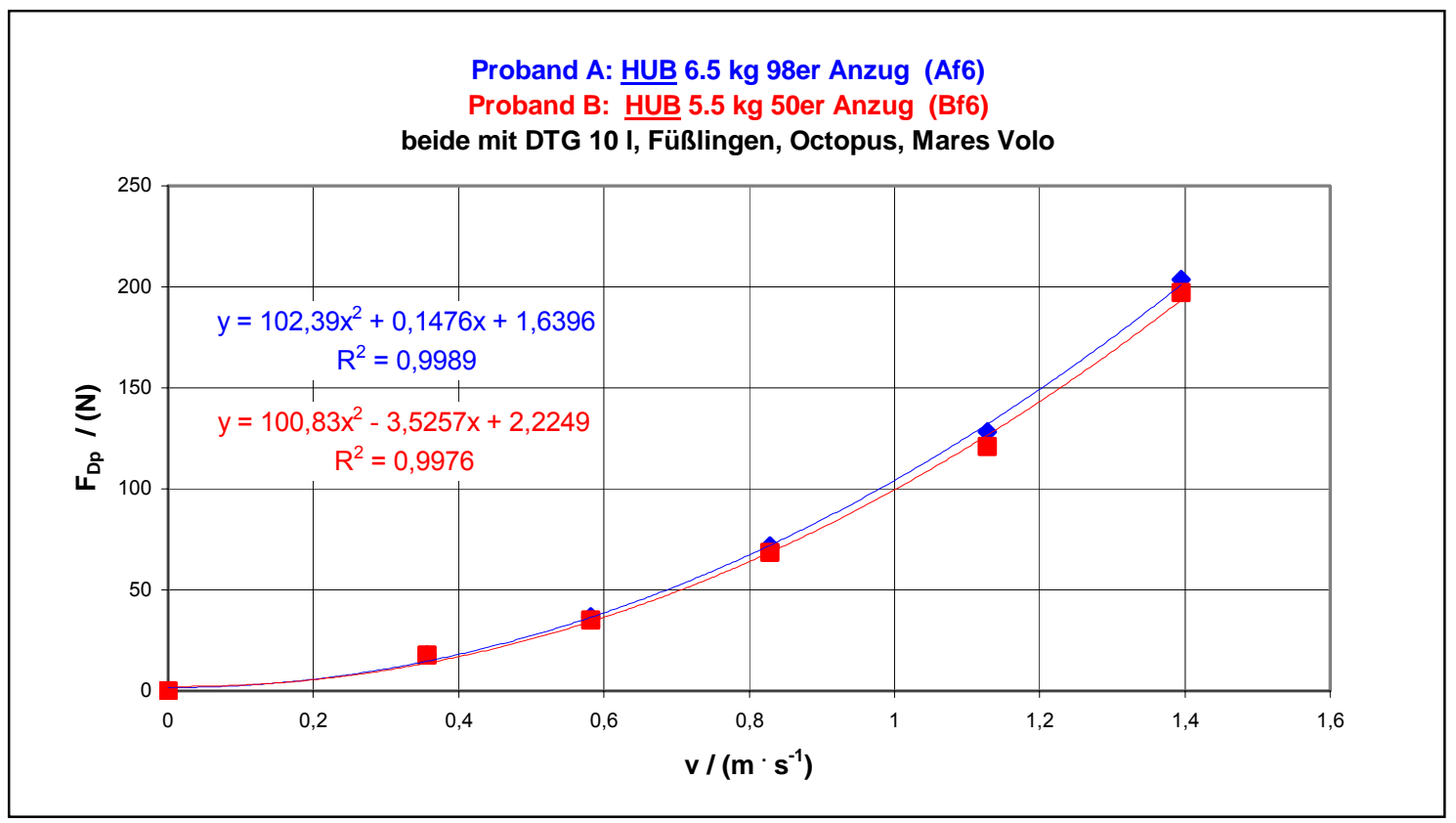

Abbildung 23: Kraftwerte $\mathrm{F}_{\mathrm{Dp}} /(\mathrm{N})$ für beide Probanden mit dem HUB.

Die Kraftwerte beider Probanden mit dem Jacket sind für Proband $B$ in allen Geschwindigkeitsstufen die niedrigsten gemessenen Werte. Bei Proband A trifft das auf die letzten beiden Geschwindigkeitsstufen zu. Für Proband A liegen die Werte in den ersten drei Geschwindigkeitsstufen über den Werten von Proband B mit einer Maximaldifferenz von $6,83 \mathrm{~N}$ bei $0,8289 \mathrm{~m} \cdot \mathrm{s}^{-1}$. Die Kurven nähern sich auf $2,96 \mathrm{~N}$ bei $1,1283 \mathrm{~m} \cdot \mathrm{s}^{-1}$ und $3,11 \mathrm{~N}$ bei $1,3952 \mathrm{~m} \cdot \mathrm{s}^{-1}$ wieder an.

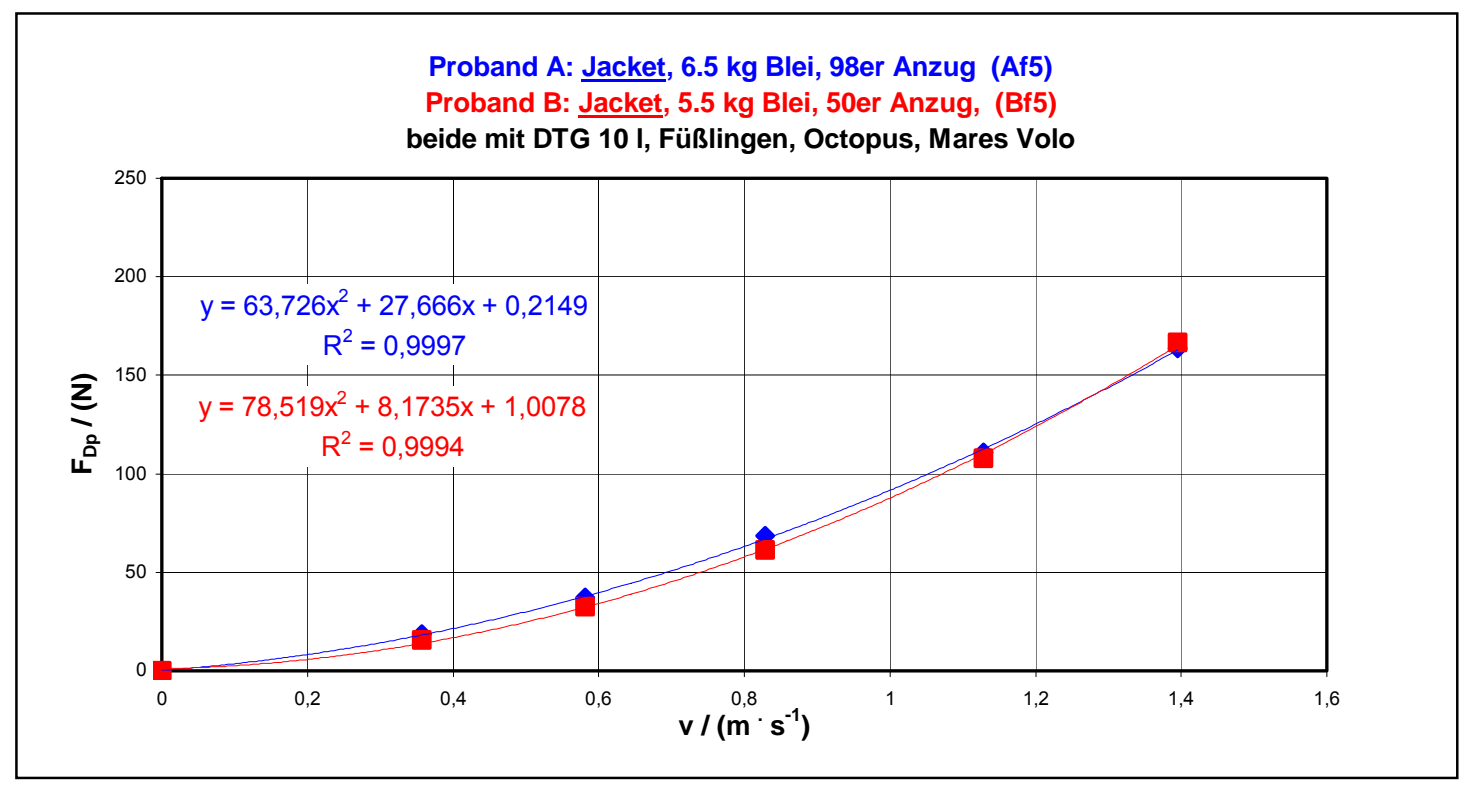

Abbildung 24: Kraftwerte $\mathrm{F}_{\mathrm{Dp}} /(\mathrm{N})$ für beide Probanden mit dem Jacket. 


\subsubsection{Gegenüberstellung aller Kraftwerte je Proband}

Deutlich wird, dass besonders unter Bedingungen starker Strömung, resp. starker Geschwindigkeit, die geschwommen werden soll, der Wert von $F_{D p}$ stark zunimmt. Die Gegenüberstellung der Kraftwerte für die einzelnen Auftriebskörper zeigt eine deutliche Ähnlichkeit der Abstufung der Kurven bei beiden Probanden. Da die Kurvenverläufe der Ausrüstungsgegenstände bei beiden Probanden ähnlich sind, ist zu erwarten, dass die Darstellung aller Ausrüstungsgegenstände für jeden Probanden die gleiche Abstufung der Kraftkurven ergibt.

Bei Proband A liegen die Kurven für die Auftriebskörper in den Geschwindigkeitsstufen $0,3567 \mathrm{~m} \cdot \mathrm{s}^{-1}$ und $0,5817 \mathrm{~m} \cdot \mathrm{s}^{-1}$ eng beieinander, auch wenn die Kragenweste hier eindeutig das Maximum bestimmt. Ab 0,8289 m $\mathrm{s}^{-1}$ teilen sich die Kurven. Das Jacket und das Wing Jacket haben mit einem internen Abstand von 7,17 N bei 1,3952 $\mathrm{m} \cdot \mathrm{s}^{-1}$ die niedrigeren Kraftwerte. Kragenweste und HUB liegen mit einem internen Abstand von 4,98 $\mathrm{N}$ bei $1,3952 \mathrm{~m} \cdot \mathrm{s}^{-1}$ ebenfalls eng zusammen. Der Abstand zwischen der Kragenweste und dem Wing Jacket beträgt bei $1,3952 \mathrm{~m} \cdot \mathrm{s}^{-1} 27,77 \mathrm{~N}$.

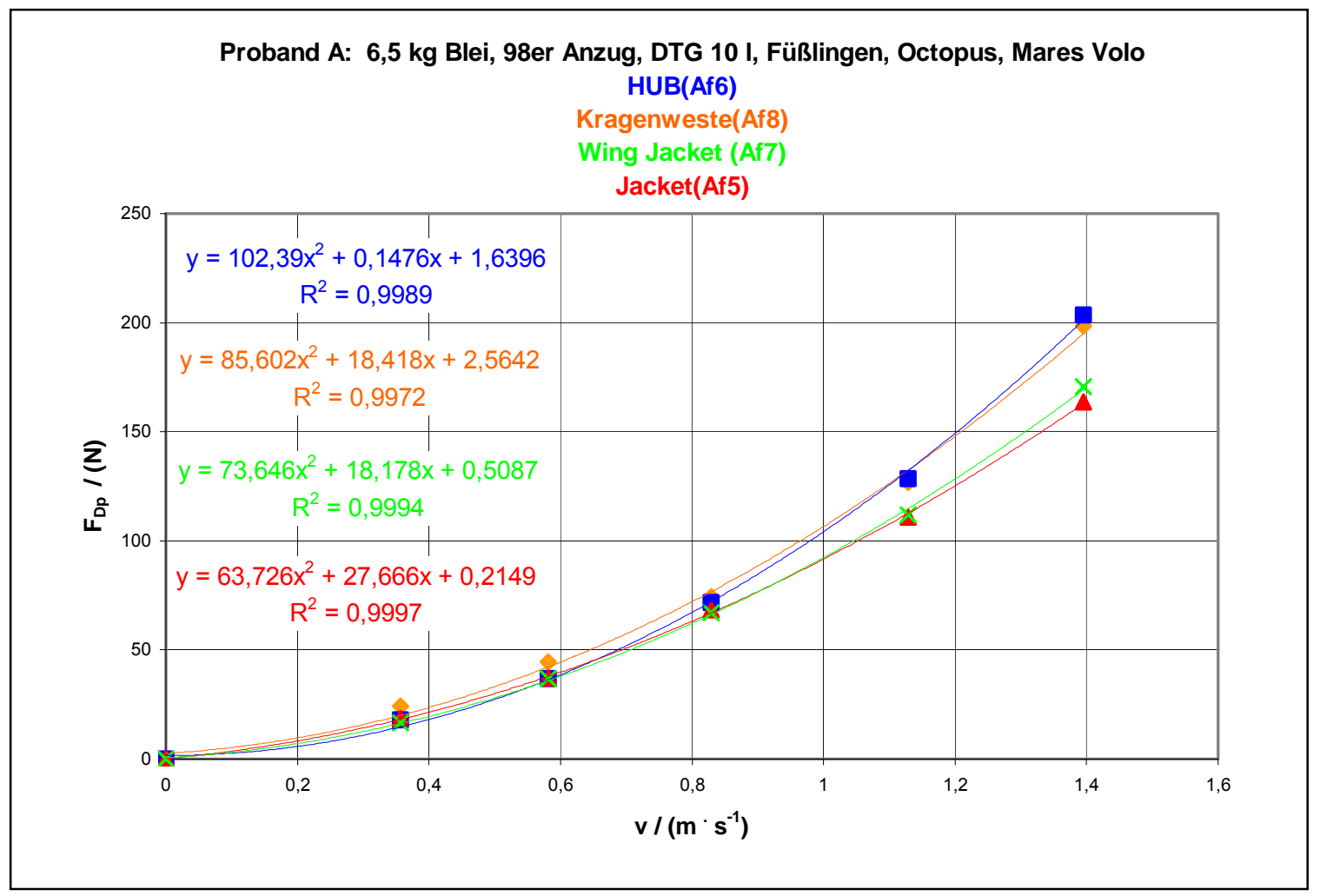

Abbildung 25: Widerstandswerte $F_{D p} /(N)$ aller Auftriebskörper für Proband $A$.

Auch bei Proband B liegen die Kurven für die Auftriebskörper in den Geschwindigkeitsstufen $0,3567 \mathrm{~m} \cdot \mathrm{s}^{-1}$ und $0,5817 \mathrm{~m} \cdot \mathrm{s}^{-1}$ eng beieinander. Auch hier bestimmt die Kragenweste das Maximum. Ab $0,8289 \mathrm{~m} \cdot \mathrm{s}^{-1}$ trennen sich die Kurvenverläufe. Das Jacket und das Wing Jacket haben mit einem internen Abstand von $6,45 \mathrm{~N}$ bei $1,3952 \mathrm{~m} \cdot \mathrm{s}^{-1}$ die niedrigeren Kraftwerte. Es folgt die Kragenweste mit einem um 8,33 $\mathrm{N}$ höheren Kraftwert als das Wing Jacket. Den höchsten Kraftwert hat 
das HUB mit einem Abstand von $15,57 \mathrm{~N}$ bei $1,3952 \mathrm{~m} \cdot \mathrm{s}^{-1}$ gegenüber der Kragenweste.

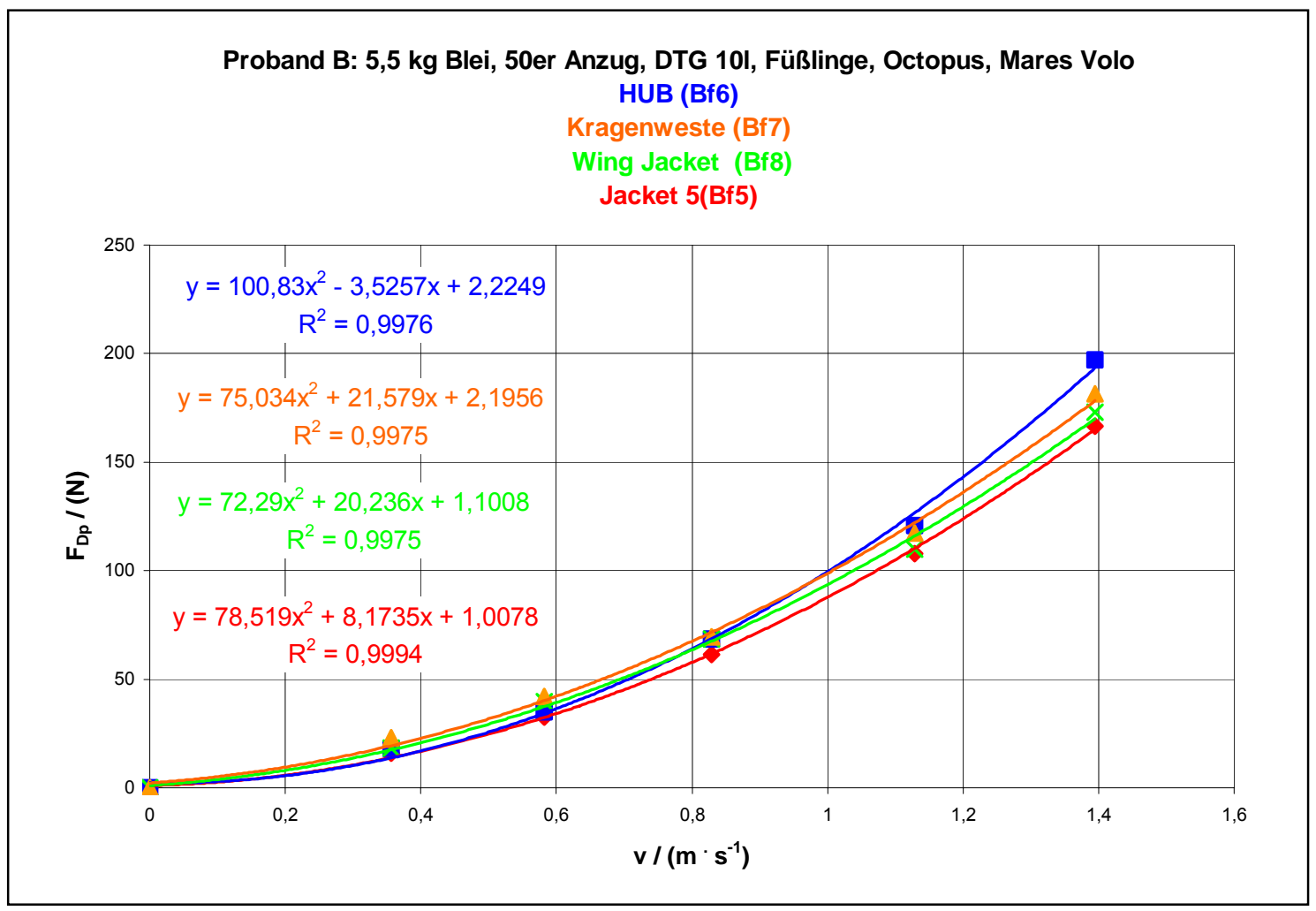

Abbildung 26: Widerstandswerte $\mathrm{F}_{\mathrm{Dp}} /(\mathrm{N})$ aller Auftriebskörper für Proband $\mathrm{B}$.

\subsubsection{Errechnete mechanische Leistung}

Da Kraftwerte und Geschwindigkeit bekannt sind, kann mit

$$
P_{\text {mech }}=F_{D p} \cdot v
$$

direkt die mechanische Leistung in Watt berechnet werden, welche die Probanden beim freien Schwimmen hätten erbringen müssen, um der angeströmten Geschwindigkeit Stand halten zu können und damit auf der Stelle stehen zu bleiben. Dabei bleibt die Gesamtleistung $P$ unberücksichtigt. Auf sie kann mit dieser Verfahrensweise auch nicht direkt geschlossen werden. Jedoch kann die mechanische Leistung $P_{\text {mech }}$ aus den vorhanden Kraftwerten entsprechend Formel 23 berechnet werden.

Die absoluten und relativen Steigerungsraten sind analog den in Kap. 5.2.1 vorgestellten Ergebnissen zu sehen, da $F_{D p}$ jeweils mit $v$ multipliziert wird, um $P$ zu ermitteln und $v$ auf jeder Geschwindigkeitsstufe konstant ist.

Aus darstellungstechnischen Gründen sind die Stufen in der umgekehrten Reihenfolge benannt. Rechts ist die niedrigste Geschwindigkeitsstufe und nach links die folgenden bis zur höchsten Geschwindigkeitsstufe. 


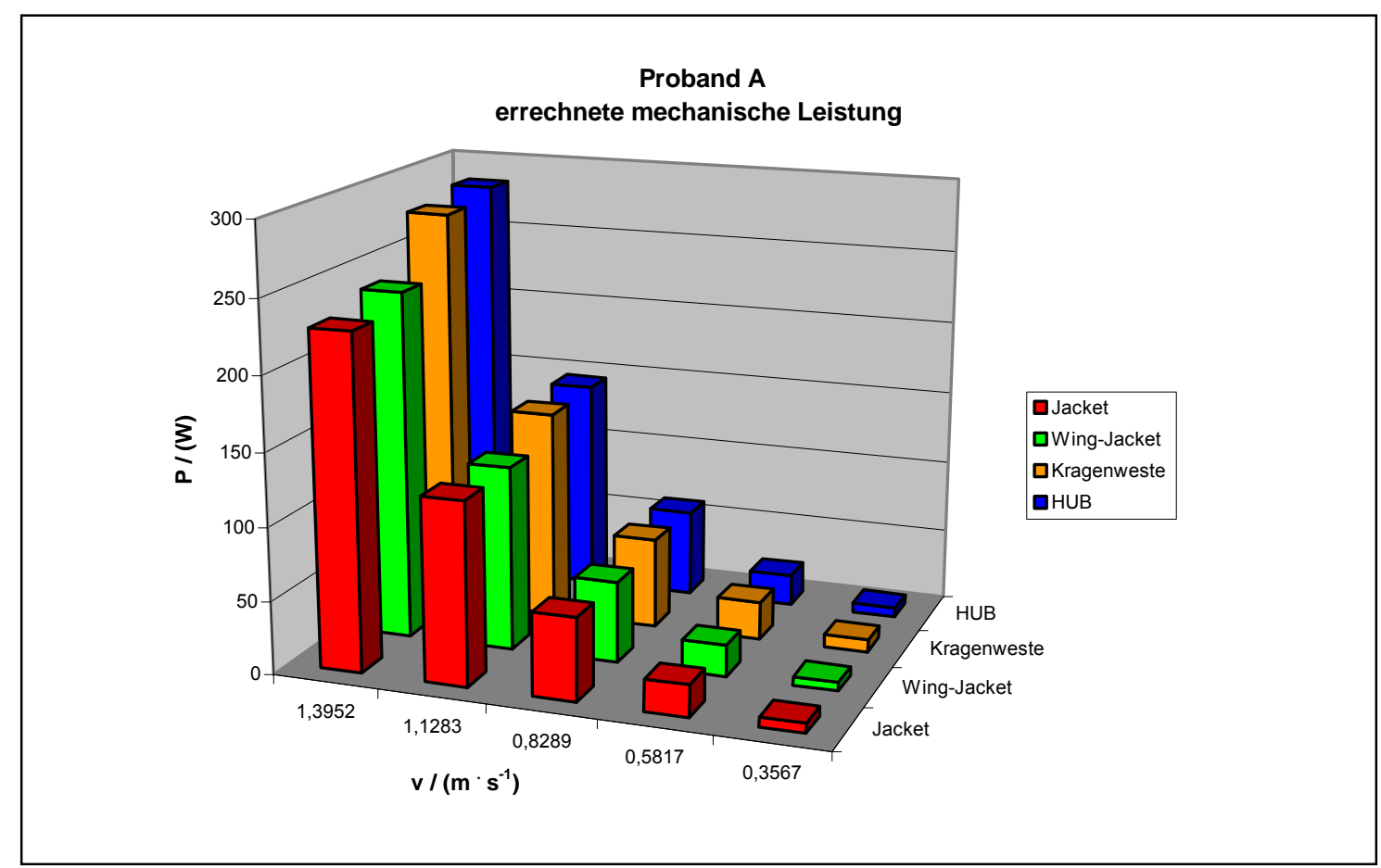

Abbildung 27: Errechnete mechanische Leistung je Auftriebskörper für Proband A.

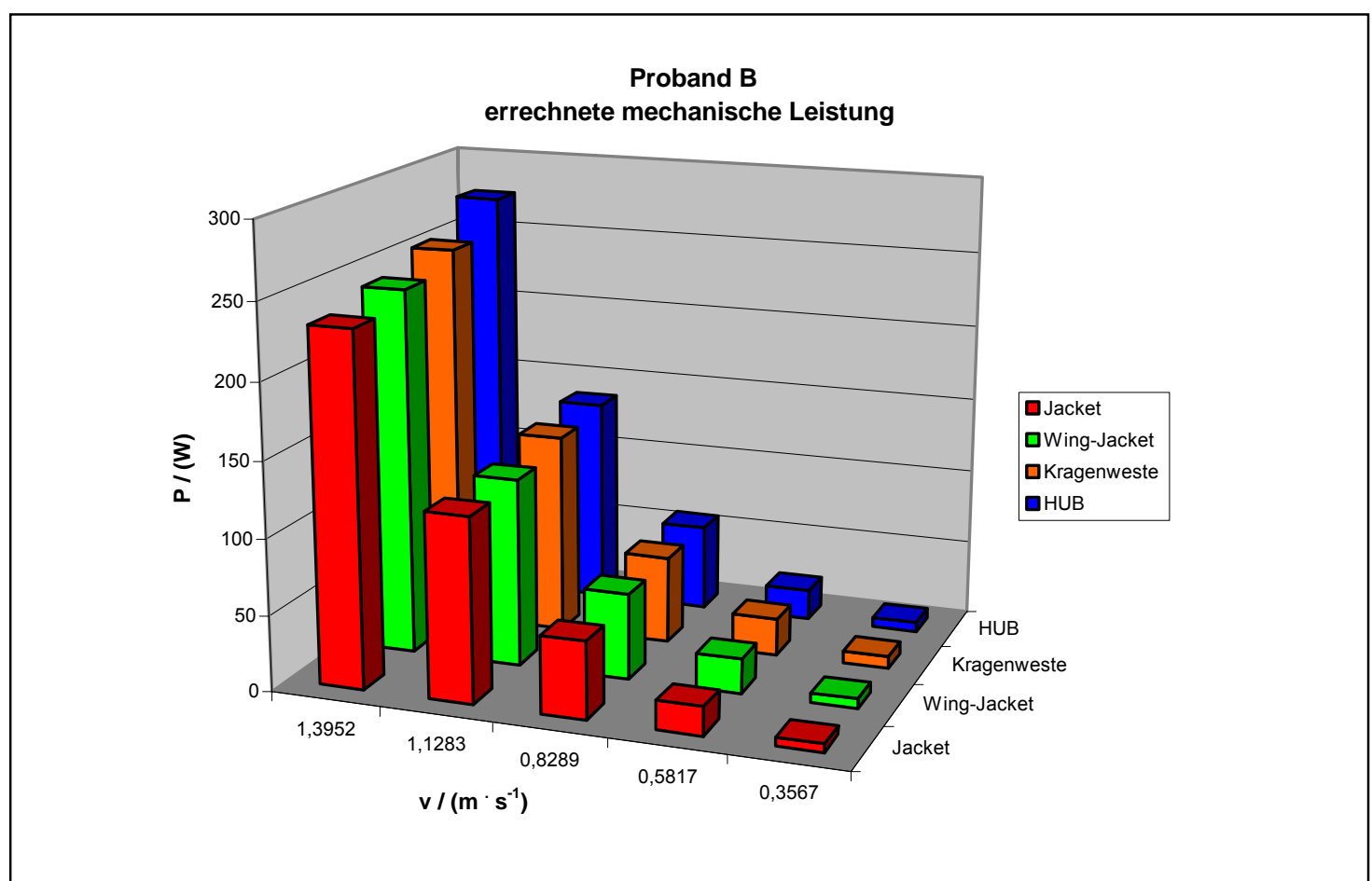

Abbildung 28: Errechnete mechanische Leistung je Auftriebskörper für Proband B.

In Tabelle 9 sind die Mittelwerte der jeweiligen Leistungsstufen der beiden Probanden dargestellt. Es wird deutlich, dass diese mit $\pm 0,11$ Watt bis $\pm 3,14$ Watt eng beieinander liegen, wie folgerichtig auch die prozentuale Abweichung mit $\pm 1,24 \%- \pm 2,42 \%$ gering ist. Es fällt auf, dass die stärksten absoluten Abweichungen in der 4. und 5 Stufe liegen, während in den ersten beiden Stufen die Unterschiede mit 0,11 und 0,45 Watt verschwindend gering sind. Die stärkste relative Abweichung liegt bei der mittleren 
Geschwindigkeit mit 2,42 \%, während das relative Minimum hier in der höchsten Geschwindigkeitsstufe zu errechnen ist.

\begin{tabular}{|c|c|c|c|c|}
\hline Geschwindigkeit & Proband A & Proband B & Differenz in \% & Differenz \\
\hline $\mathrm{v} /\left(\mathrm{m} \cdot \mathrm{s}^{-1}\right)$ & $\mathrm{P} /(\mathrm{W})$ & $\mathrm{P} /(\mathrm{W})$ & zum Mittelwert & {$[\mathrm{W}]$} \\
\hline 1,3952 & 256,81 & 250,52 & $\pm 1,24$ & $\pm 3,14$ \\
\hline 1,1283 & 134,84 & 128,72 & $\pm 2,32$ & $\pm 3,06$ \\
\hline 0,8289 & 58,30 & 55,55 & $\pm 2,42$ & $\pm 1,38$ \\
\hline 0,5817 & 22,60 & 21,71 & $\pm 2,01$ & $\pm 0,45$ \\
\hline 0,3567 & 6,91 & 6,68 & $\pm 1,68$ & $\pm 0,11$ \\
\hline
\end{tabular}

Tabelle 9: $\quad$ Mittelwerte $P$ je Proband je Geschwindigkeitsstufe. Differenzwerte in Prozent und Watt.

In den beiden folgenden Tabellen sind die Differenz zur durchschnittlichen Bruttoleistung in Watt dargestellt, die für die unterschiedlichen Auftriebskörper bei den beiden Probanden ermittelt wurden. Die jeweiligen Maximalwerte sind hervorgehoben und das Maximum der Differenz errechnet und auf der rechten Seite dargestellt.

\begin{tabular}{|c|c|c|c|c|c|}
\hline Proband A & & & & & \\
\hline Geschwindigkeit & Jacket & Wing Jacket & Kragenweste & HUB & \begin{tabular}{|l} 
Max. Differenz \\
\end{tabular} \\
\hline $\mathrm{v} /\left(\mathrm{m} \cdot \mathrm{s}^{-1}\right)$ & $\mathrm{P}_{\text {mech }} /(\mathrm{W})$ & $\mathrm{P}_{\text {mech }} /(\mathrm{W})$ & $\mathrm{P}_{\text {mech }} /(\mathrm{W})$ & $\mathrm{P}_{\text {mech }} /(\mathrm{W})$ & $(\mathrm{W})$ \\
\hline 1,3952 & $-25,47$ & $-15,46$ & 23,27 & 30,22 & 55,69 \\
\hline 1,1283 & $-6,70$ & $-5,65$ & 11,48 & 13,12 & 19,81 \\
\hline 0,8289 & $-0,29$ & $-1,44$ & 4,74 & 2,48 & 6,18 \\
\hline 0,5817 & $-0,53$ & $-0,71$ & 3,79 & $-0,76$ & 4,55 \\
\hline 0,3567 & $-0,12$ & $-0,83$ & 1,85 & $-0,44$ & 2,68 \\
\hline
\end{tabular}

Tabelle 10: Differenz zur durchschnittlichen errechneten mechanischen Leistung in Watt bei Proband A.

\begin{tabular}{|c|c|c|c|c|c|}
\hline \multicolumn{6}{|l|}{ Proband B } \\
\hline Geschwindigkeit & Jacket & Wing Jacket & Kragenweste & HUB & Max. Differenz \\
\hline $\mathrm{v} /\left(\mathrm{m} \cdot \mathrm{s}^{-1}\right)$ & $\mathrm{P}_{\text {mech }} /(\mathrm{W})$ & $\mathrm{P}_{\text {mech }} /(\mathrm{W})$ & $\mathrm{P}_{\text {mech }} /(\mathrm{W})$ & $\mathrm{P}_{\text {mech }} /(\mathrm{W})$ & $(\mathrm{W})$ \\
\hline 1,3952 & $-21,13$ & $-12,13$ & $-0,52$ & 21,21 & 42,34 \\
\hline 1,1283 & $-10,03$ & $\begin{array}{l}-7,58 \\
\end{array}$ & 0,80 & 4,58 & 14,61 \\
\hline 0,8289 & $-5,95$ & $-0,14$ & 0,82 & $-0,23$ & 6,77 \\
\hline 0,5817 & $-3,23$ & 0,86 & 2,39 & $-1,80$ & 5,63 \\
\hline 0,3567 & $-1,15$ & $-0,29$ & 1,42 & $-0,44$ & 2,56 \\
\hline
\end{tabular}

Tabelle 11: Differenz zur durchschnittlichen errechneten mechanischen Leistung in Watt bei Proband B.

Tabelle 10 und 11 zeigen, dass im Bereich der in der Literatur angegebenen Werte einer normalen Geschwindigkeit für Sporttaucher HOFFMANN (2002), SCHLAG AUF SCHLAG $(1997,107)$ von 0,4-0,5 $\mathrm{m} \cdot \mathrm{s}^{-1}$, entsprechend Stufe 1 und 2 nur geringe Differenzen bis maximal 5,63 Watt auftreten, die auch in der 3. Geschwindigkeitsstufe bei $0,8289 \mathrm{~m} \cdot \mathrm{s}^{-1}$ mit 6,18 und 6,77 Watt annähernd konstant bleiben. Bei den drei unteren Geschwindigkeitsstufen liegen alle Maxima bei der Kragenweste. Die Minima liegen bei Proband A beim Jacket und bei Proband B in zwei Stufen beim Wing Jacket und in der 2. Stufe beim HUB. Die ersten drei Stufen zeigen das Jacket mit absolut niedrigsten Werten (Proband $B$ ) und niedrigen Werten (Proband $A$ ), das Wing Jacket und das HUB mit niedrigen Werten bei beiden Probanden und die Kragenweste mit absolut höchsten Werten. In den beiden letzten Geschwindigkeitsstufen werden die 
Unterschiede gravierend, bis zu 55,69 Watt, und die Zuordnung ist bei beiden Probanden eindeutig. Das Jacket hat die absolut günstigsten Werte. Das Wing Jacket hat niedrige Werte und das HUB hat die höchsten Werte. Die Werte für die Kragenweste sind bei Proband $A$ fast im Bereich des sehr ungünstigen HUB, während sie bei Proband B niedrig sind.

\subsection{Die Strömungswiderstände $F_{D p}$ der Probanden mit verschiedenen Flossen (Messreihe 3)}

Für Proband A werden, entsprechend der unter 3.4 beschriebenen Vorgehensweise, maximal 1,339 \% einer Versuchsreihe (Mares Volo/0,3567 $\mathrm{m} \cdot \mathrm{s}^{-1}$ ) und bei Proband B maximal $0,483 \%$ einer Versuchsreihe (Monoflosse/0,5817 $\mathrm{m} \cdot \mathrm{s}^{-1}$ ) nach diesem Verfahren ausgeschlossen. Die Raten liegen in ihrer Gesamtheit unter dem Grenzwert von $2 \%$ für den Maximalausschluss.

Mittelwert und Median ${ }^{23}$ liegen für Mares Volo, Sporasub Dessault und Seemann Sub SF 2 hinreichend exakt beieinander. Die Abweichungen betragen bei Proband $A$ maximal $-0,07 \mathrm{~N}$ und $+0,80 \mathrm{~N}$ und bei Proband $\mathrm{B}-0,35 \mathrm{~N}$ und $+1,00 \mathrm{~N}$. Bei beiden Probanden hat die Monoflosse in den ersten drei Geschwindigkeitsstufen hohe Abweichungen von $+1,20 \mathrm{~N},+2,89 \mathrm{~N}$ und $+0,97 \mathrm{~N}$ bei Proband $\mathrm{A}$ und $+0,83 \mathrm{~N},+1,20 \mathrm{~N}$ und $+1,36 \mathrm{~N}$ bei Proband $\mathrm{B}$. Die Schiefe ${ }^{24}$, als Maß für die Normalverteilung jedes Datensatzes, liegt zwischen $-0,03$ und $+0,81$ für Proband $A$ und zwischen $-0,27$ und $+1,06$ für Proband $B$.

Im Folgenden sind die gemessenen Kraftwerte für beide Probanden tabellarisch aufgeführt. Die Kraftwerte steigen mit zunehmender Geschwindigkeit an.

\begin{tabular}{|l|c|c|c|c|c|}
\hline Proband A & $0,3567 /\left(\mathrm{m} \cdot \mathrm{s}^{-1}\right)$ & $0,5817 /\left(\mathrm{m} \cdot \mathrm{s}^{-1}\right)$ & $0,8289 /\left(\mathrm{m} \cdot \mathrm{s}^{-1}\right)$ & $1,1283 /\left(\mathrm{m} \cdot \mathrm{s}^{-1}\right)$ & $1,3951 /\left(\mathrm{m} \cdot \mathrm{s}^{-1}\right)$ \\
\hline & $\mathrm{F}_{\mathrm{Dp}} /(\mathrm{N})$ & $\mathrm{F}_{\mathrm{Dp}} /(\mathrm{N})$ & $\mathrm{F}_{\mathrm{Dp}} /(\mathrm{N})$ & $\mathrm{F}_{\mathrm{Dp}} /(\mathrm{N})$ & $\mathrm{F}_{\mathrm{Dp}} /(\mathrm{N})$ \\
\hline Mares Volo & 10,77 & 20,08 & 33,63 & 55,34 & 83,53 \\
\hline Seemann Sub SF 2 & 20,88 & 23,49 & 31,30 & 49,42 & 66,25 \\
\hline Sporasub Dessault & 14,55 & 18,10 & 30,56 & 47,96 & 73,73 \\
\hline Monoflosse & 28,91 & 31,18 & 42,08 & 48,93 & 64,20 \\
\hline
\end{tabular}

Tabelle 12: $\quad$ Kraftwerte mit Flossen für Proband $A$

\begin{tabular}{|l|c|c|c|c|c|}
\hline Proband B & $0,3567 /\left(\mathrm{m} \cdot \mathrm{s}^{-1}\right)$ & $0,5817 /\left(\mathrm{m} \cdot \mathrm{s}^{-1}\right)$ & $0,8289 /\left(\mathrm{m} \cdot \mathrm{s}^{-1}\right)$ & $1,1283 /\left(\mathrm{m} \cdot \mathrm{s}^{-1}\right)$ & $1,3951 /\left(\mathrm{m} \cdot \mathrm{s}^{-1}\right)$ \\
\hline & $\mathrm{F}_{\mathrm{Dp}} /(\mathrm{N})$ & $\mathrm{F}_{\mathrm{Dp}} /(\mathrm{N})$ & $\mathrm{F}_{\mathrm{Dp}} /(\mathrm{N})$ & $\mathrm{F}_{\mathrm{Dp}} /(\mathrm{N})$ & $\mathrm{F}_{\mathrm{Dp}} /(\mathrm{N})$ \\
\hline Mares Volo & 10,96 & 20,61 & 45,17 & 64,57 & 84,53 \\
\hline Seemann Sub SF 2 & 11,34 & 14,64 & 25,17 & 44,85 & 58,96 \\
\hline Sporasub Dessault & 9,05 & 15,38 & 25,91 & 39,49 & 64,35 \\
\hline Monoflosse & 18,06 & 21,34 & 33,75 & 45,97 & 68,07 \\
\hline
\end{tabular}

Tabelle 13: $\quad$ Kraftwerte mit Flossen für Proband B.

${ }^{23}$ Die Tabellen für den Vergleich zwischen Median und Mittelwert sind im Anhang 8.1.8 .

${ }^{24}$ Die Tabellen für die Schiefe und vier exemplarische Diagramme der Verteilung sind im Anhang 8.1.9 und 8.1.10 . Die Messwerte sind auf der CD-ROM in den Dateien: „Kraftwerte_ Flossen_Proband_A.xIs“ und „Kraftwerte_Flossen_Proband_B.xls“. 
Die Bestimmtheitsmaße liegen im Bereich von 0,9782 und 0,9986, wobei es einen Ausreißer bei der Monoflosse von Proband A mit 0,9628 gibt.

\begin{tabular}{|l|c|c|}
\hline & $\mathrm{R}^{2}$ Proband A & $\mathrm{R}^{2}$ Proband B \\
\hline Mares Volo & 0,9986 & 0,9841 \\
\hline Seemann SUB SF 2 & 0,9782 & 0,9927 \\
\hline Sporasub Dessault & 0,9902 & 0,9923 \\
\hline Monoflosse & 0,9628 & 0,9841 \\
\hline
\end{tabular}

Tabelle 14: $\quad$ Bestimmtheitsmaß der Kraftkurven jeder Flosse.

Im ersten Schritt wurden analog zum Versuchsaufbau bei der Widerstandsmessung mit unterschiedlichen Auftriebskörpern die Widerstandskräfte für die Flossen bestimmt. Im Folgenden werden entsprechend der beabsichtigten Vorgehensweise die Mittelwerte dargestellt, die innerhalb der 4-fachen positiven und negativen Standardabweichung liegen. Auf die Gegenüberstellung der gleichen Flossen je Proband wird verzichtet.

Bei Proband A weisen in der polynomischen Darstellung alle Kraftwerte der Flossen einen exponentiellen Verlauf auf, mit Ausnahme der Monoflosse. Die Kurve der Monoflosse hat mit $-11,698$ als einzige eine negativen Exponention.

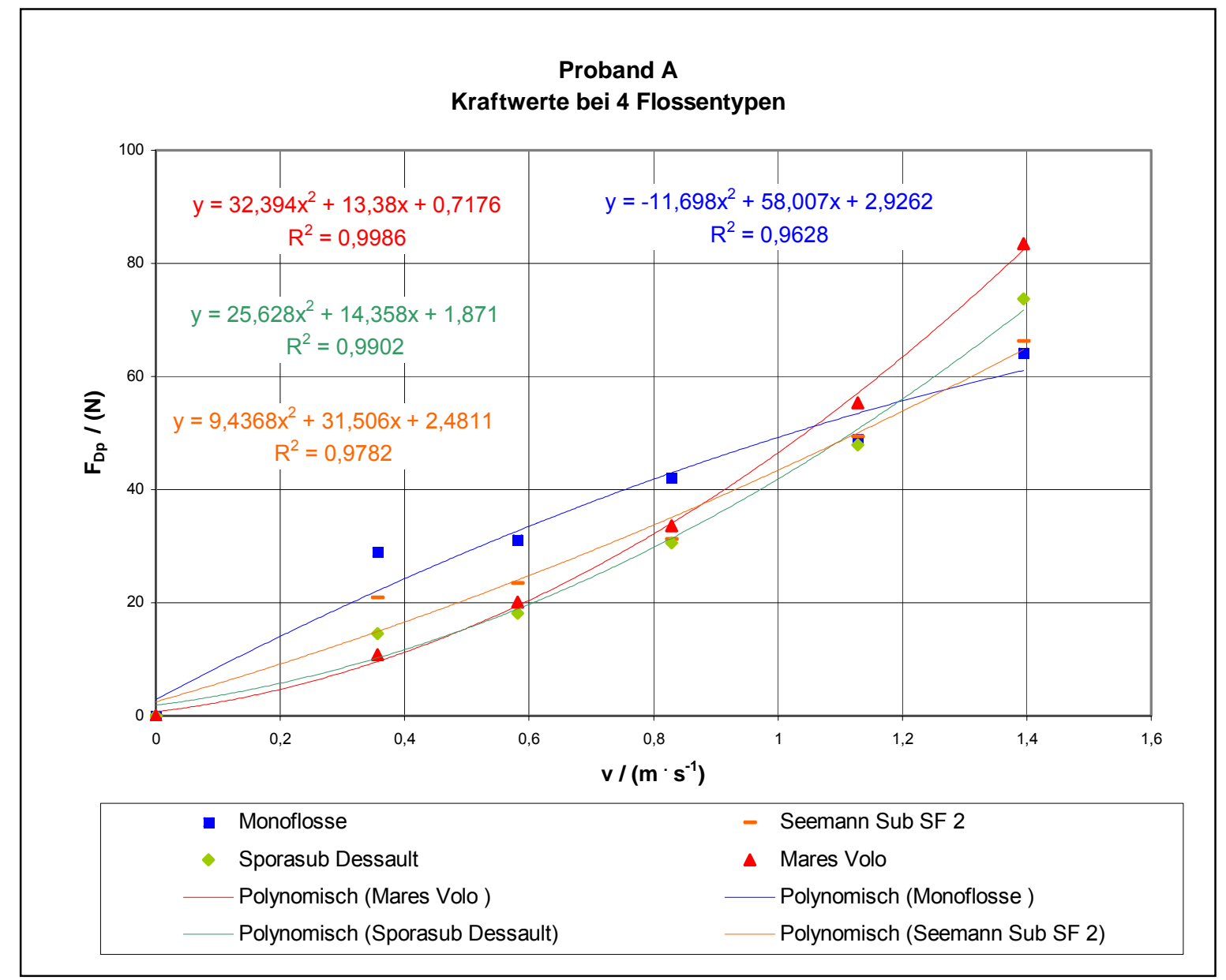

Abbildung 29: Polynomische Darstellung der Kraftwerte $F_{D p} /(N)$ von Proband A mit vier unterschiedlichen Flossentypen. 
Die Kurven für die Mares Volo und die Sporasub Dessault sind in den ersten beiden Geschwindigkeitsstufen annähernd deckungsgleich und liegen niedriger als die Kurve der Seemann Sub SF 2. In der dritten Stufe nähern sich alle Kurve an und ab dieser Stufe ist die Mares Volo die Flosse mit den höchsten Widerstandswerten. In der 4. Geschwindigkeitsstufe haben die Sporasub Dessault und die Seemann Sub SF 2 fast identische Werte. In der letzten Stufe ist die Seemann Sub SF 2 die vom Widerstand her günstigere Flosse.

Die Filmdokumente bestätigen für Proband $A$ in allen Stufen deutliche Veränderungen in der Haltung, was die Schwankungsbreite der Kraftwerte erklärt. Abweichungen von einer strömungsgünstigen Haltung führen bei Proband A zu einer deutlichen Erhöhung der Messwerte für die beiden Geschwindigkeitsstufen. Die Versuchsreihe Monoflosse wird für die folgende Messreihe 4 nicht weitergeführt, weil die erhobenen Schleppwiderstände nicht realistisch sind und die Einflussgrößen außerhalb des Untersuchungsrahmens liegen.

Bei Proband B weisen in der polynomischen Darstellung alle Kraftwerte der Flossen einen positiven exponentiellen Verlauf auf.

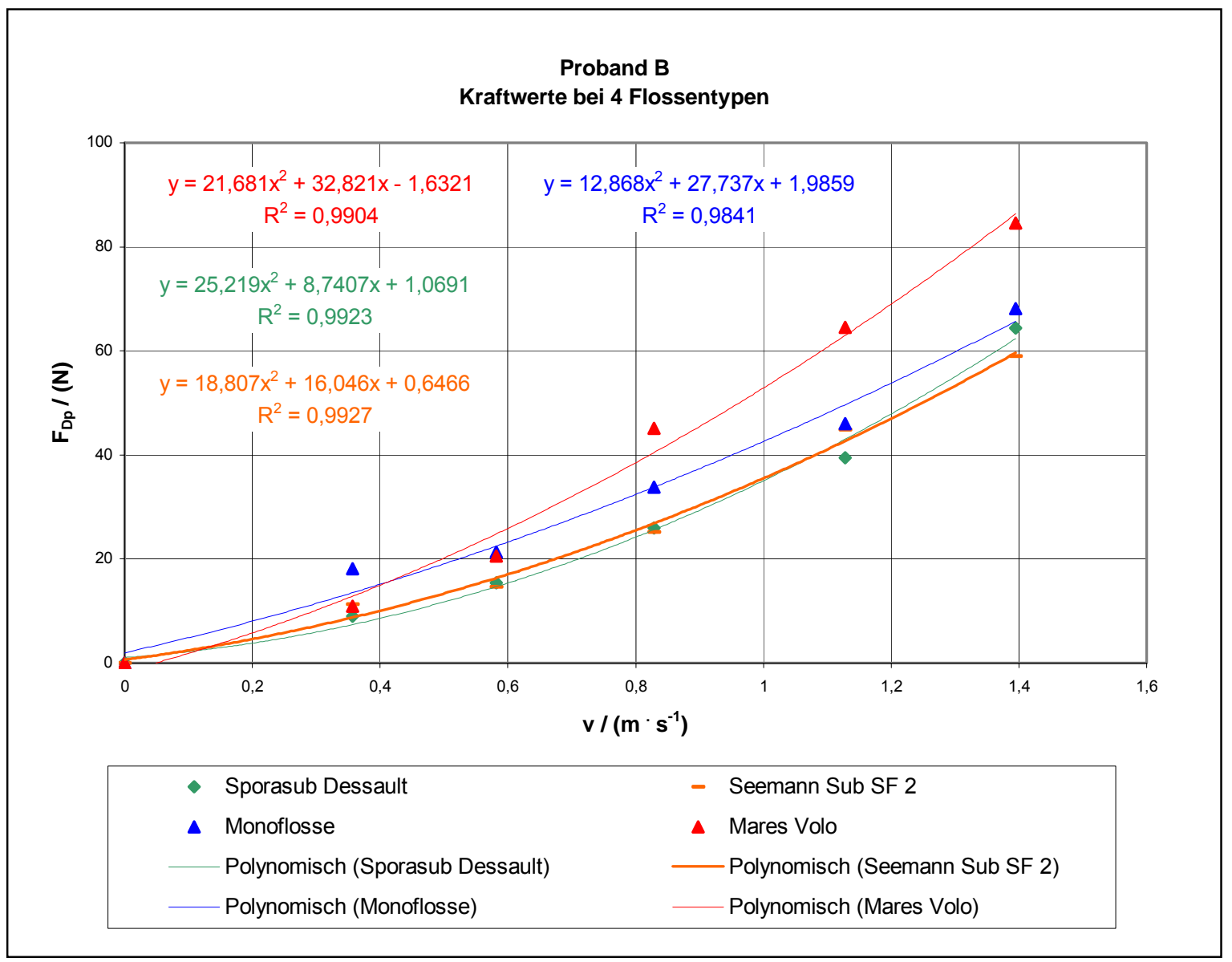

Abbildung 30: Polynomische Darstellung der Kraftwerte $F_{D p} /(N)$ von Proband B mit vier unterschiedlichen Flossentypen.

Bei Proband B ist die Mares Volo in der 3. - 5. Geschwindigkeitsstufe die Flosse mit dem höchsten Widerstandswerten. Sie erklären sich aus der Haltung des Probanden, 
der die Knie in diesen Stufen ca. $80^{\circ}$ nach oben abwinkelte und somit von der strömungsgünstigen Haltung abwich. In der ersten Stufe ist die Monoflosse ungünstiger. In der zweiten Stufe liegen diese beiden Flossen gleichauf. In den Videoauswertungen konnte festgestellt werden, dass die Monoflosse in den ersten beiden Geschwindigkeitsstufen bedingt durch ihre Form und Größe nach unten hing. Dadurch wurde, so ist zu vermuten, die angeströmte Fläche vergrößert, was im Ergebnis zu den höheren Werten führte. Die Seemann Sub SF 2 und die Sporasub Dessault haben fast identische Kurven und ähnliche Kraftwerte. Erst in der letzten Stufe ist die Seemann Sub SF 2 etwas günstiger als die Sporasub Dessault, welche dabei dann knapp unterhalb der Monoflosse liegt.

\subsection{Spiroergometrische Daten erhoben mit den Flossen aus der vorgenannten Messreihe (Messreihe 4)}

In diesem Kapitel werden alle Daten, die spiroergometrisch erhoben wurden, dargestellt wie RQ, Netto- und Bruttowirkungsgrad sowie Netto- und Bruttoleistung. Auf den dynamischen Widerstand und MET wird am Ende des Kapitels eingegangen.

\subsubsection{Anmerkungen zu Vorgehensweise und Versuchsabläufen}

Exemplarisch wird die Vorgehensweise anhand der Daten und $\mathrm{VO}_{2}-$ Kurve von Proband A mit der Seemann Sub SF 2 erläutert. Danach wird auf die Daten und die Kurve von Proband B mit der Mares Volo eingegangen, die eine Besonderheit enthält. Die Kurven sind unterlegt mit einer Ablaufleiste, die auf die geplante Vorgehensweise in Kapitel 3.3.4 Bezug nimmt und Abweichungen darstellt. Abgebildet sind die Messpunkte $\dot{V} \mathrm{O}_{2}$ $\left[\mathrm{ml} \cdot \mathrm{min}^{-1}\right]$, die alle 15 Sekunden gefunden wurden.

Der Versuch Proband A mit Seemann Sub SF 2 verlief wie geplant. Die Belastungsstufen waren drei Minuten lang. Unterbrochen wurden sie von jeweils einer Minute passiven Schleppens. Zu Beginn jeder Belastungsstufe ist ein Anstieg des VO2 zu beobachten. Der Wert des $\dot{V} \mathrm{O}_{2}$ nimmt in den ersten drei Belastungsstufen nach einem Einschwingverhalten von ca. einer bis 1,5 Minuten einen stabilen Wert ein. In der letzten Belastungsstufe sind in der Endphase Schwankungen zu beobachten. $\dot{V} \mathrm{O}_{2}$ steigt im Vergleich zur vorhergehenden Belastungsstufe an. 


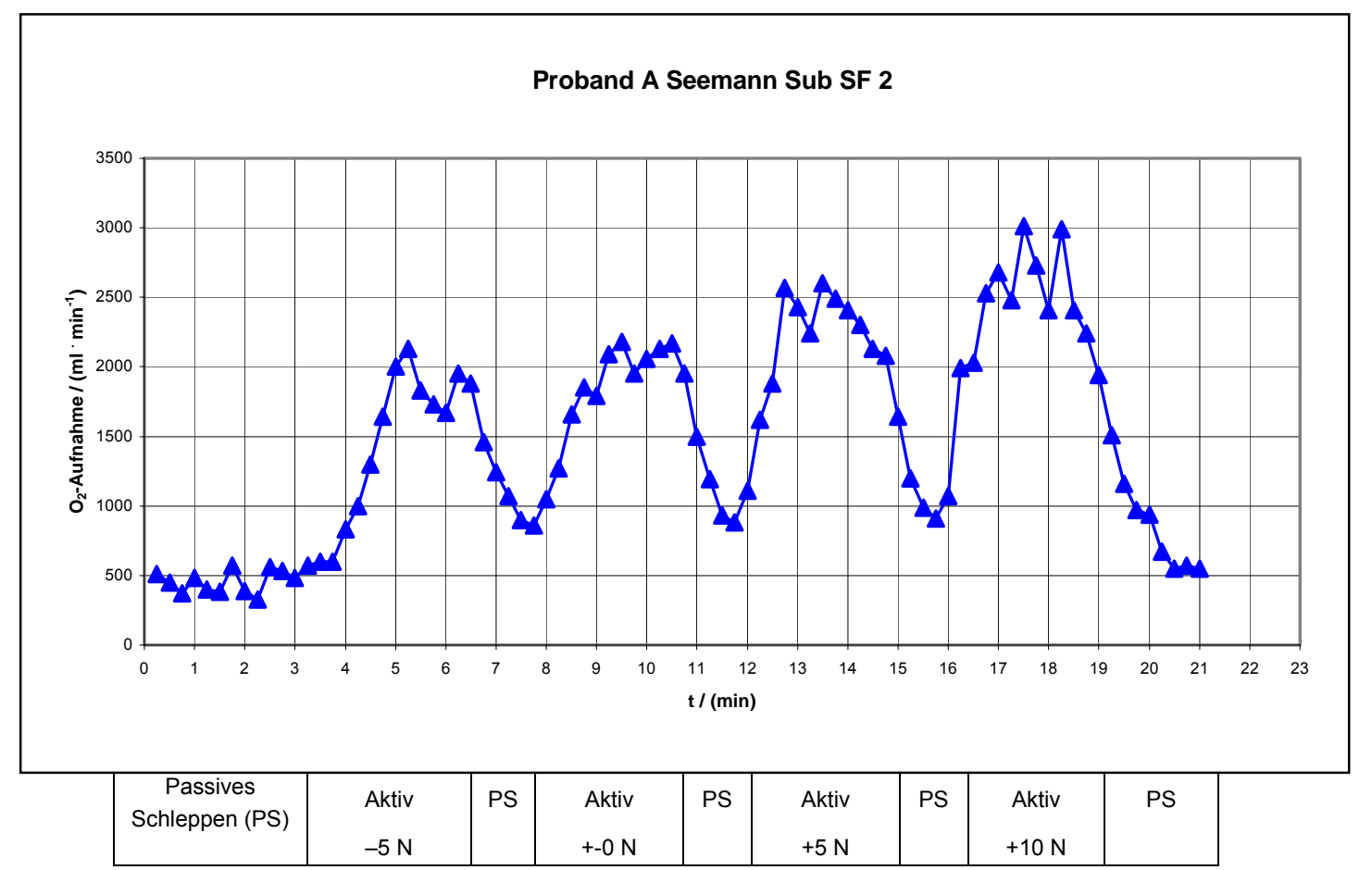

Abbildung 31: Versuchsablauf Proband A mit Seemann Sub SF 2.

Die Daten des $\mathrm{VO}_{2}$ die bei Proband $\mathrm{B}$ mit der Monoflosse erhoben wurden, zeigen einen mit den Daten von Proband A mit der Seemann Sub SF 2 vergleichbaren Verlauf. Das Einschwingverhalten ist in der ersten und dritten Belastungsstufe zu erkennen. In der zweiten Belastungsstufe ist es kaum ausgeprägt. In der letzten Belastungsstufe sind die Schwankungen extrem. Sie betragen in der maximalen Ausprägung $1510 \mathrm{ml} \cdot \mathrm{min}^{-1}$ in der Zeit von 17:45 min auf 18:00 min. Der niedrige Wert von 17:45 min liegt unterhalb aller Werte der zweiten Belastungsstufe.

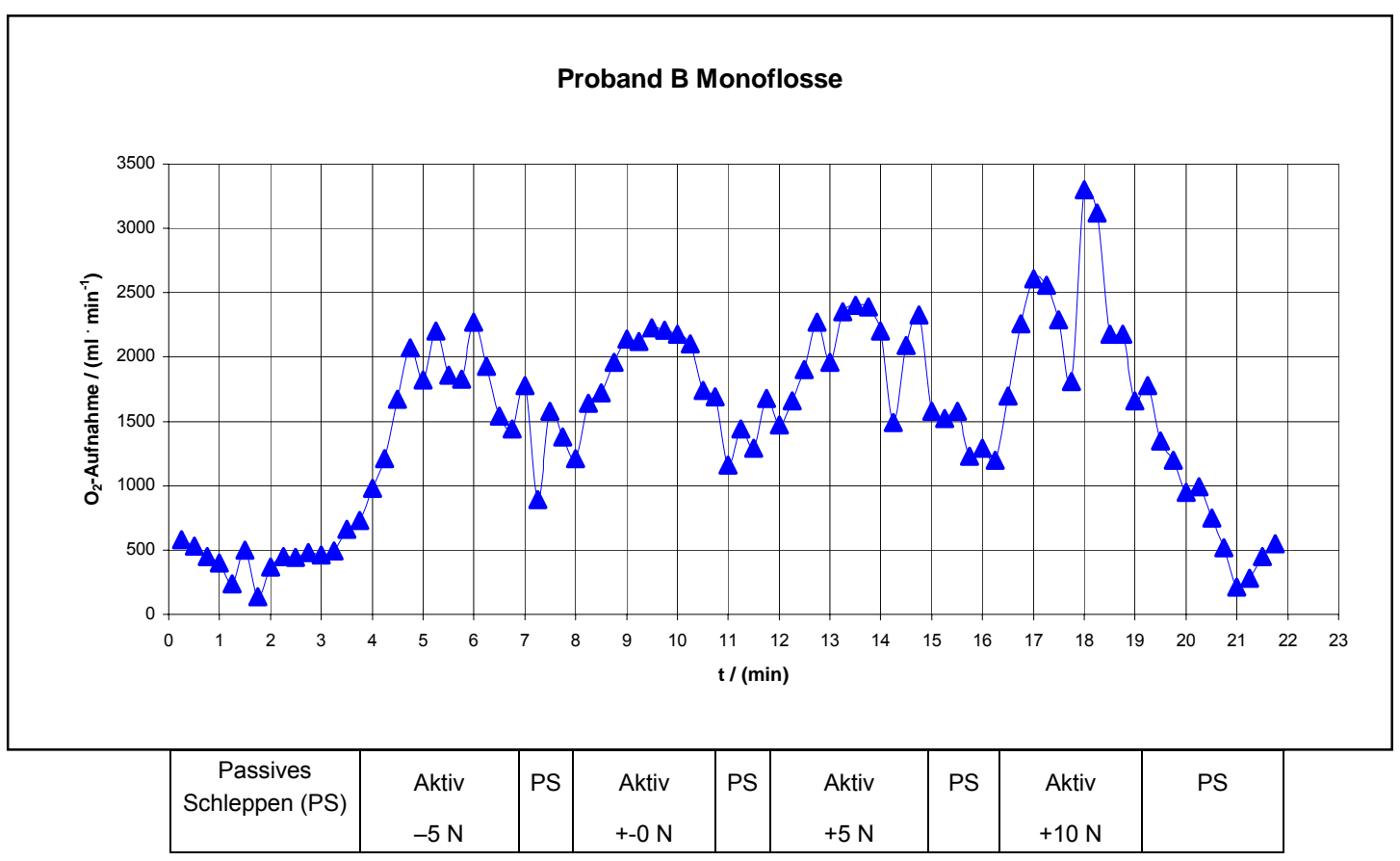

Abbildung 32: Versuchsablauf Proband B mit Monoflosse. 
Der Versuch Proband B mit der Mares Volo verlief bis zum Beginn der 3. Belastungsstufe regulär. Nach Minute 14:30 trat ein Defekt am Atemregler auf. Einer der Schläuche zur Ableitung der Ausatemluft rutschte vom Atemregler ab und der Versuch musste unterbrochen werden. Da die nötigen Korrekturen innerhalb von zwei Minuten vorgenommen werden konnten, wurde der Versuch weitergeführt. Die dritte Belastungsstufe wurde wiederholt, da bis zum Abbruch nicht genügend Daten erhoben werden konnten. Gestartet wurde mit einer zweiminütigen Phase des passiven Schleppens. Aus dieser Besonderheit ergibt sich eine Verlängerung des Versuchs um 5 Minuten auf ein Ende des Versuchs bei 27 Minuten.

Die Daten zeigen das Einschwingverhalten in allen vier Belastungsstufen. Für Stufe eins werden alle Werte zwischen Minuten 4:45 und 7:00 berücksichtigt. Stufe zwei wird mit Werten zwischen Minute 9:30 und 11:30 dargestellt. Vor Stufe drei lag der Abbruch des Versuches wegen der genannten Probleme mit dem Atemregler. Für Stufe drei sind die Werte zwischen Minuten 20:15 und 21:30 und für Stufe vier der Bereich von Minute 22:30 bis Minute 26:45.

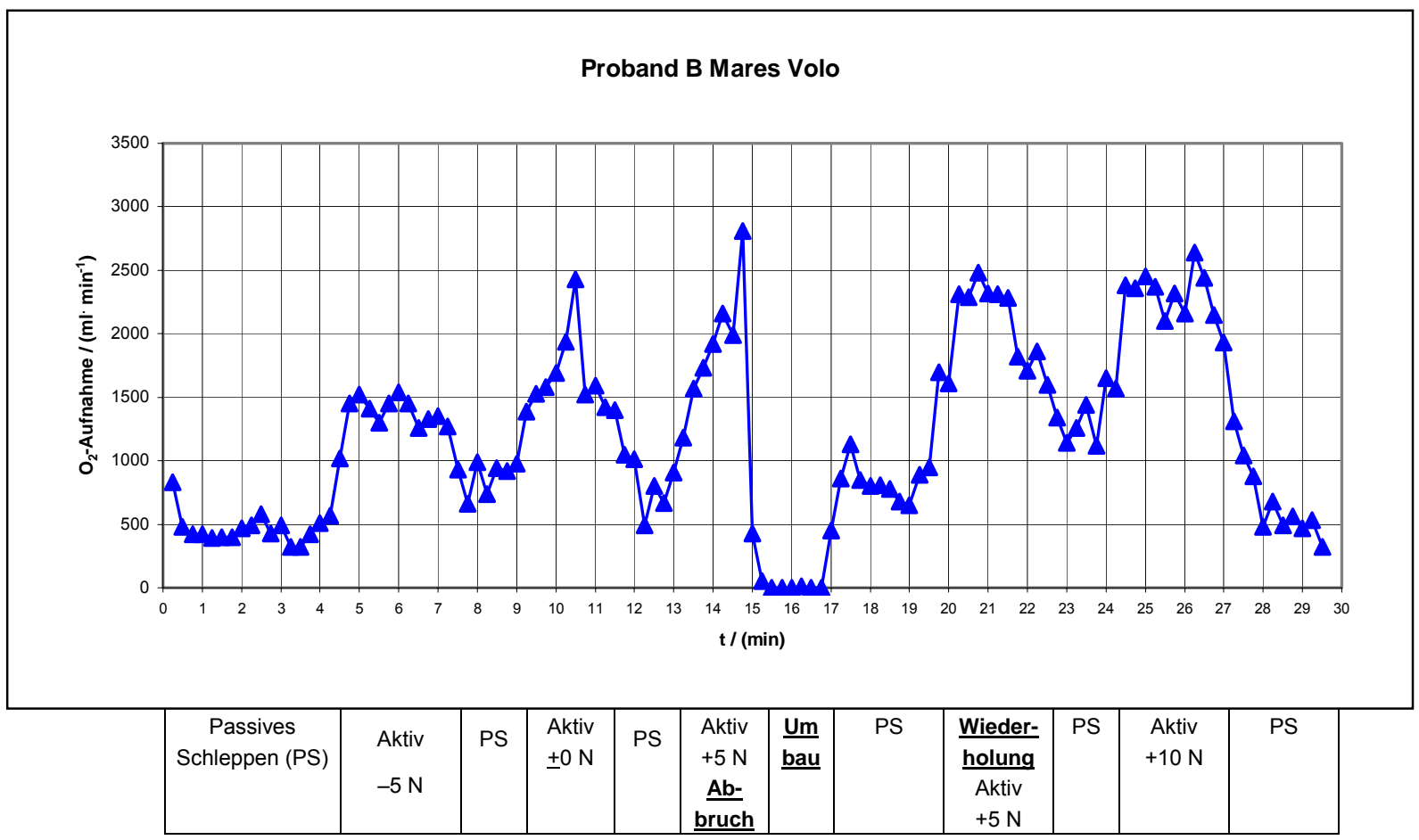

Abbildung 33: Versuchsablauf Proband B Mares Volo.

Das gleiche Problem trat beim Versuch Proband A Mares Volo auf. Es konnte nach einer Minute behoben werden. Der Versuch verlängerte sich entsprechend.

Die Kurven aller erhobenen Daten zeigen ein Einschwingverhalten der gemessenen $\mathrm{O}_{2}$ Werte für jeden Probanden. In allen Abläufen ist die Antwortreaktion auf die ansteigende Belastung im Versuchsablauf zu erkennen ${ }^{25}$.

${ }^{25}$ Die Kurven befinden sich in Anhang 8.1.11 und die Messdaten in Anhang 8.1.12 und 8.1.13 . 


\subsubsection{Prüfung des Einschwingverhaltens}

Beispielhaft wird am Versuch Proband A mit der Seemann Sub SF 2 der Vergleich aller Messwerte mit den Werten nach dem Einschwingverhalten dargestellt.

Basierend auf allen erhobenen Werten einer Belastungsstufe weist das Bestimmtheitsmaß mit $R_{\text {polynomisch }}^{2}=0,4208$ bzw. $R_{\text {linear }}^{2}=0,403$ auf hohe Ungenauigkeiten hin. In der Abbildung sind Werte zu finden, welche deutlich unterhalb der Gerade und der Kurve liegen. Diese liegen, wie der Vergleich mit der Abbildung 27 zeigt, für die ersten drei Belastungsstufen in der ersten Phase der Stufe, also in der Phase des Einschwingverhaltens. Nach Ausschluss der Werte aus der Einschwingphase ist eine hohe Genauigkeit mit $R_{\text {polynomisch }}^{2}=0,802 \mathrm{bzw} \cdot \mathrm{R}_{\text {linear }}^{2}=$ 0,8012 gegeben. $^{26}$

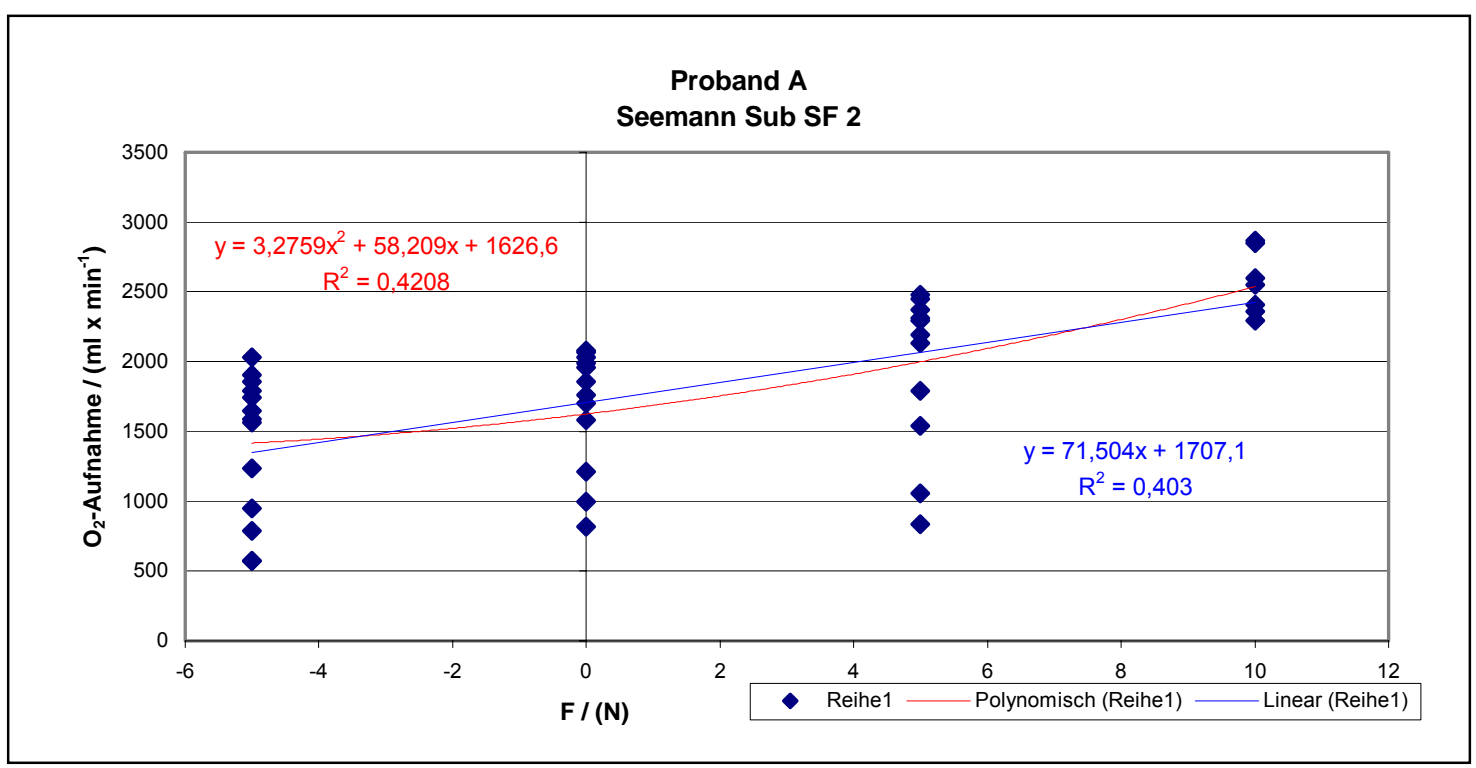

Abbildung 34: Proband A Seemann Sub SF 2, alle Werte der Belastungsstufen.

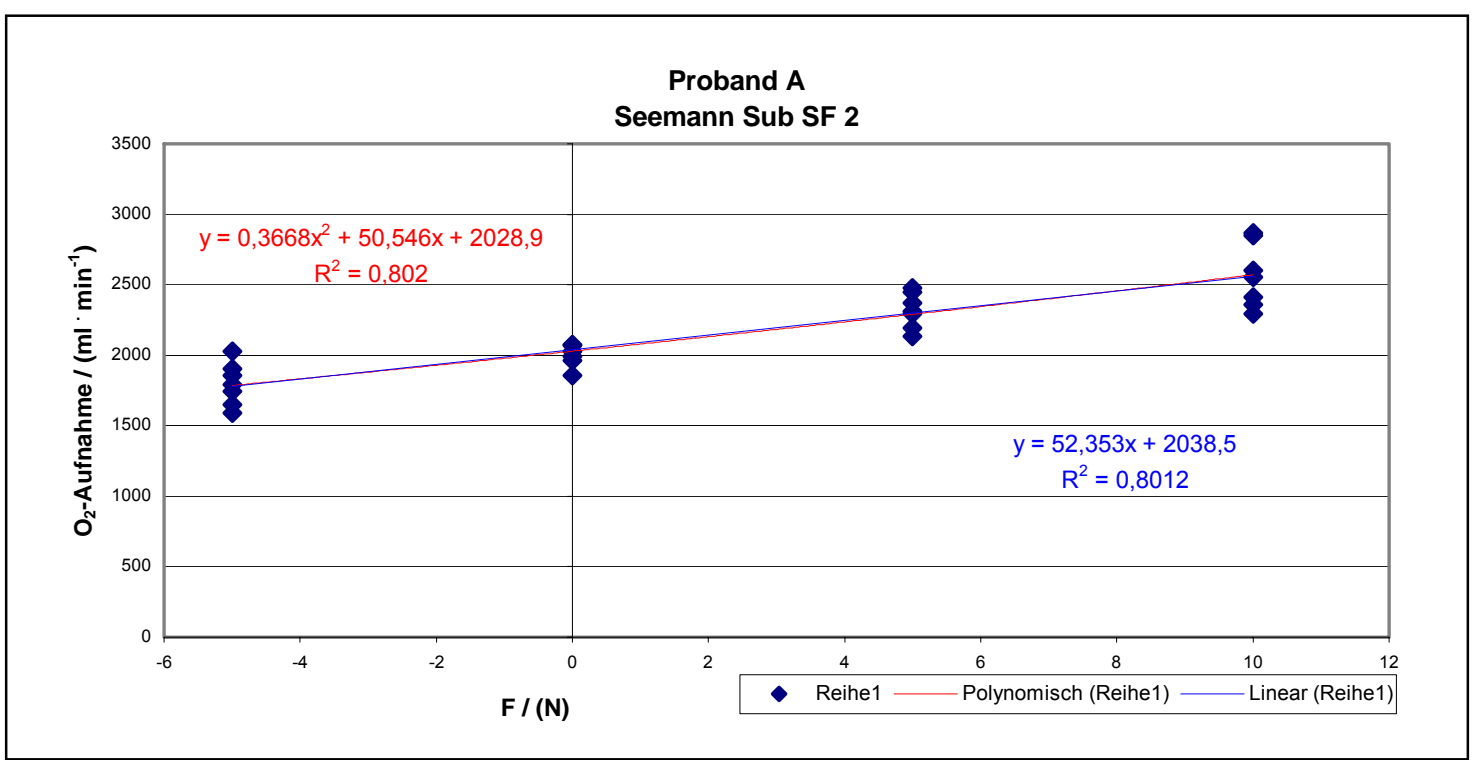

Abbildung 35: Proband A Seemann Sub SF 2, Werte nach der Einschwingphase.

${ }^{26}$ Die Gegenüberstellung aller anderen Versuche befindet sich im Anhang 8.1.14 . 
In der folgenden Tabelle ist die Anzahl der Werte je Belastungsstufe, je Versuch aufgeschlüsselt, die in die Auswertung eingeflossen sind.

\begin{tabular}{|c|c|c|c|c|c|c|c|}
\hline \multirow{2}{*}{$\begin{array}{c}\text { Belastungs- } \\
\text { stufe }\end{array}$} & \multicolumn{3}{|c|}{ Proband A } & \multicolumn{4}{c|}{ Proband B } \\
\cline { 2 - 8 } & Seemann Sub & Volo & Sporasub & Seemann Sub & Volo & Sporasub & Monoflosse \\
\hline$-5 \mathrm{~N}$ & 7 & 5 & 8 & 5 & 10 & 5 & 6 \\
\hline$+0 \mathrm{~N}$ & 6 & 6 & 6 & 8 & 9 & 9 & 6 \\
\hline$+5 \mathrm{~N}$ & 7 & 5 & 9 & 8 & 6 & 5 & 4 \\
\hline$+10 \mathrm{~N}$ & 7 & 6 & 3 & 6 & 6 & 7 & 8 \\
\hline
\end{tabular}

Tabelle 15: $\quad$ Anzahl der ausgewählten Messwerte in Messreihe 4.

Proband $B$ hat im Mittel eine kürzere Einschwingphase als Proband $A$, da mit 6,75 Werten je Belastungsstufe gegenüber 6,25 Werten von Proband $A$ mehr Werte in die Berechnungen einfließen können.

In allen weiteren Berechnungen wird der Mittelwerte der ausgewählten Messwerte gebildet.

Die Versuche Proband A Volo und Proband B Volo sind auf Grund eines technischen Defektes in der 3. Belastungsstufe unterbrochen worden. Mit einer ca. einminütigen Pause bei Proband A und einer ca. zweiminütigen Pause bei Proband B wurden die Versuche mit der passiven Schleppphase vor der dritten Belastungsstufe wieder aufgenommen. In beiden Versuchen ist zu erkennen, dass die Pause nach dem technischen Defekt massiven Einfluss hatte. Bei Proband A liegt der Mittelwert in der dritten Belastungsstufe deutlich unter und für Proband B deutlich über der zu erwartenden Geraden. Dieses zeigt sich auch in den ungenügenden Bestimmtheitsmaßen von 0,846 (Proband A) und 0,8949 (Proband B) in der linearen Funktion, aber auch in der polynomischen Funktion mit 0,9263 (Proband A) und 0,9236 (Proband B). Die Werte der 3. Belastungsstufe verfälschen also, bedingt durch die zusätzliche Pause, die komplette Auswertung. Aus diesem Grund werden in den beiden Fällen die Werte von $+5 \mathrm{~N}$ nicht berücksichtigt.

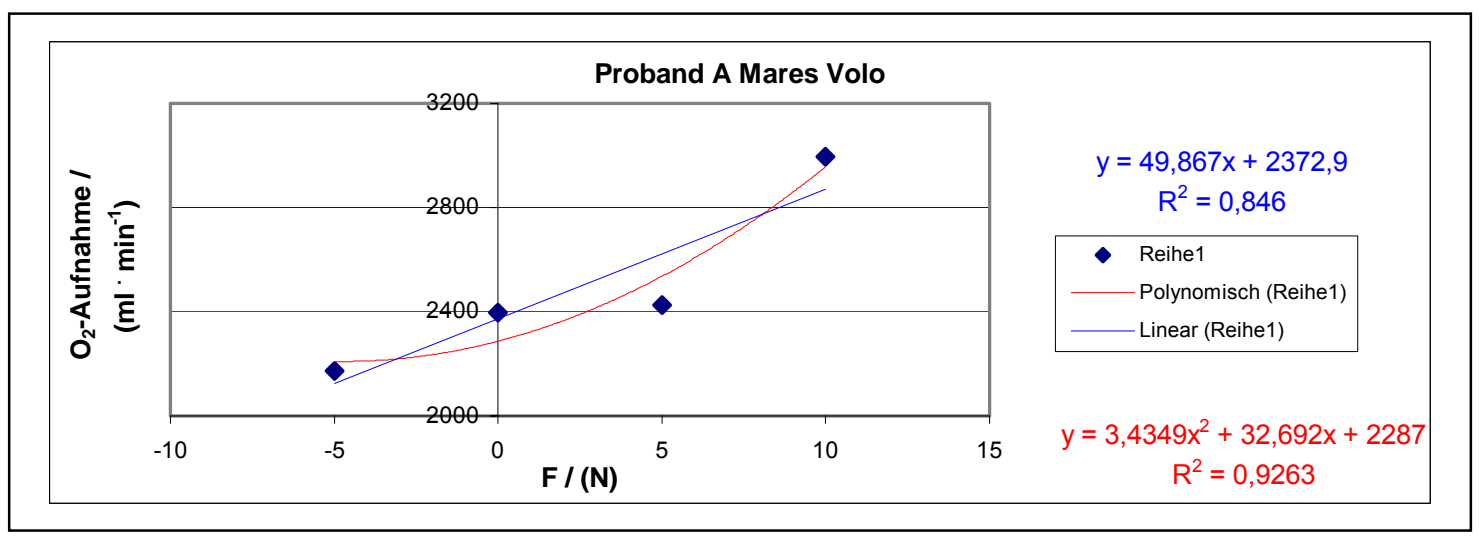

Abbildung 36: Gesamtumsatz Proband A Mares Volo. 


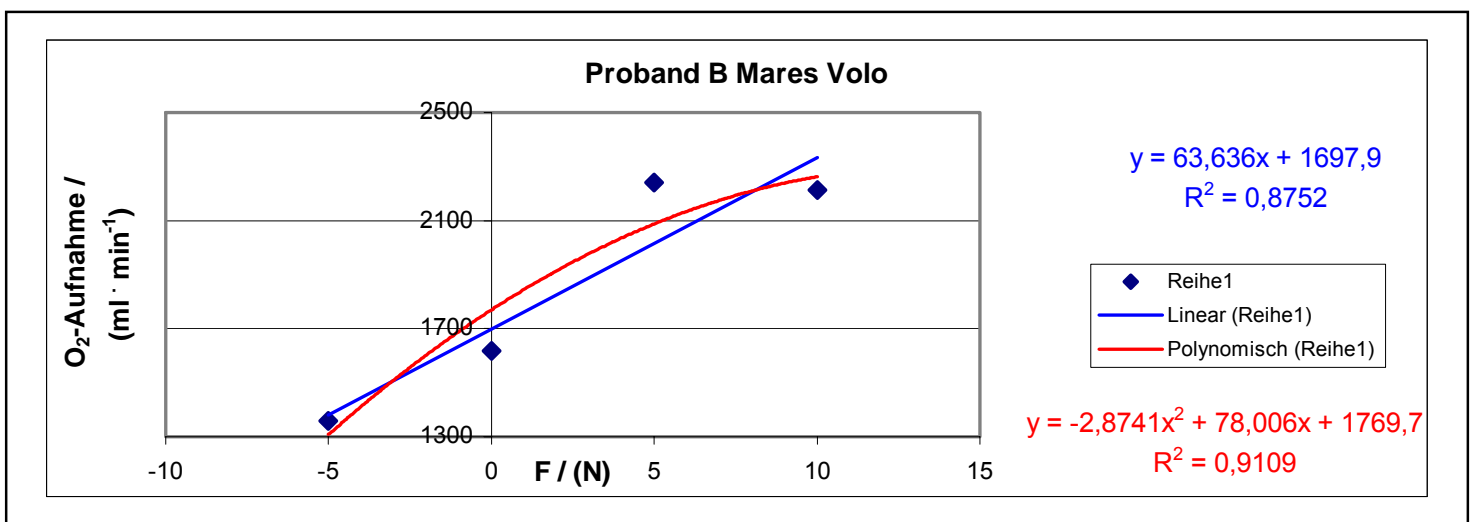

Abbildung 37: Gesamtumsatz Proband B Mares Volo.

Der niedrigere Wert von Proband A lässt sich durch eine zusätzliche Erholung erklären. Für Proband B greift diese Erklärung nicht, vor allem da der Wert sich erhöht hat. Jedoch war der Versuch von Proband B der Erste überhaupt. In diesem Fall ist die durch den technischen Defekt entstandene Unsicherheit als Grund anzuführen, da unter Wasser nicht atmen zu können die psychisch stärkste Belastung für einen Taucher darstellt. Proband A hatte es da leichter, da er vorab den Umgang mit dieser Situation von außen erlebt hat, Hilfestellung gegeben hat und wusste, wie in der Situation vom Probanden und vom Helfer zu reagieren ist.

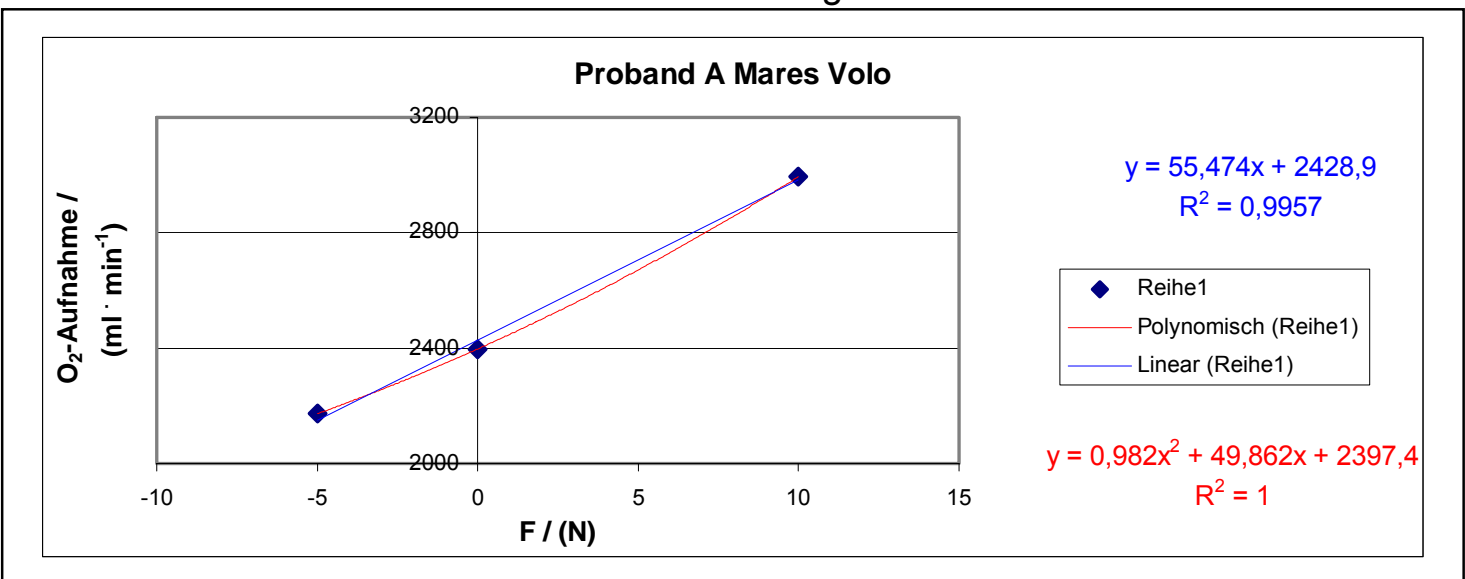

Abbildung 38: Gesamtumsatz Proband A Mares Volo ohne Stufe $3(+5 \mathrm{~N})$.

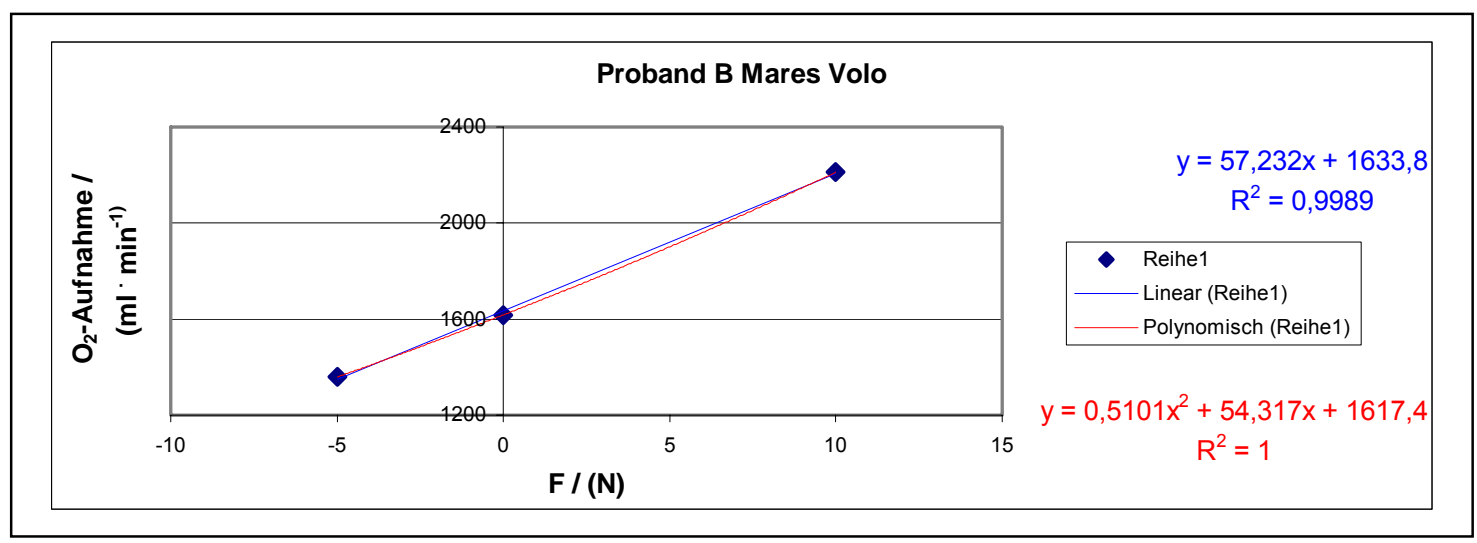

Abbildung 39: Gesamtumsatz Proband B Mares Volo ohne Stufe $3(+5 \mathrm{~N})$. 
Die folgenden Diagramme der Mittelwerte $\mathrm{VO}_{2}$ jeder Belastungsstufe weisen vom Ablauf her keine Besonderheiten auf. Die Bestimmtheitsmaße der Geraden liegen zwischen $R^{2}=0,9873$ für Proband $B$ Sporasub Dessault und $R^{2}=0,9999$ für Proband $B$ Monoflosse. Die polynomischen Bestimmtheitsmaße liegen zwischen $R^{2}=0,9944$ Proband A Seemann Sub SF 2 und $R^{2}=1$ für Proband $A$ Sporasub Dessault und Proband B Monoflosse.

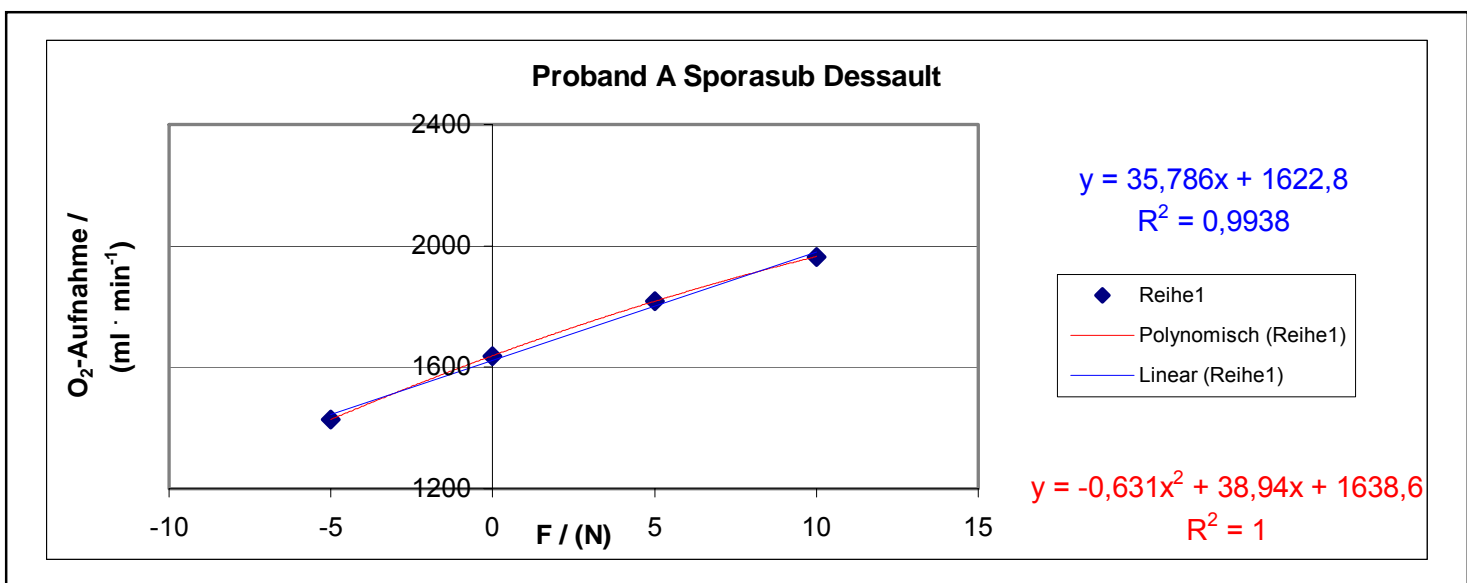

Abbildung 40: Gesamtumsatz Proband A Sporasub Dessault.

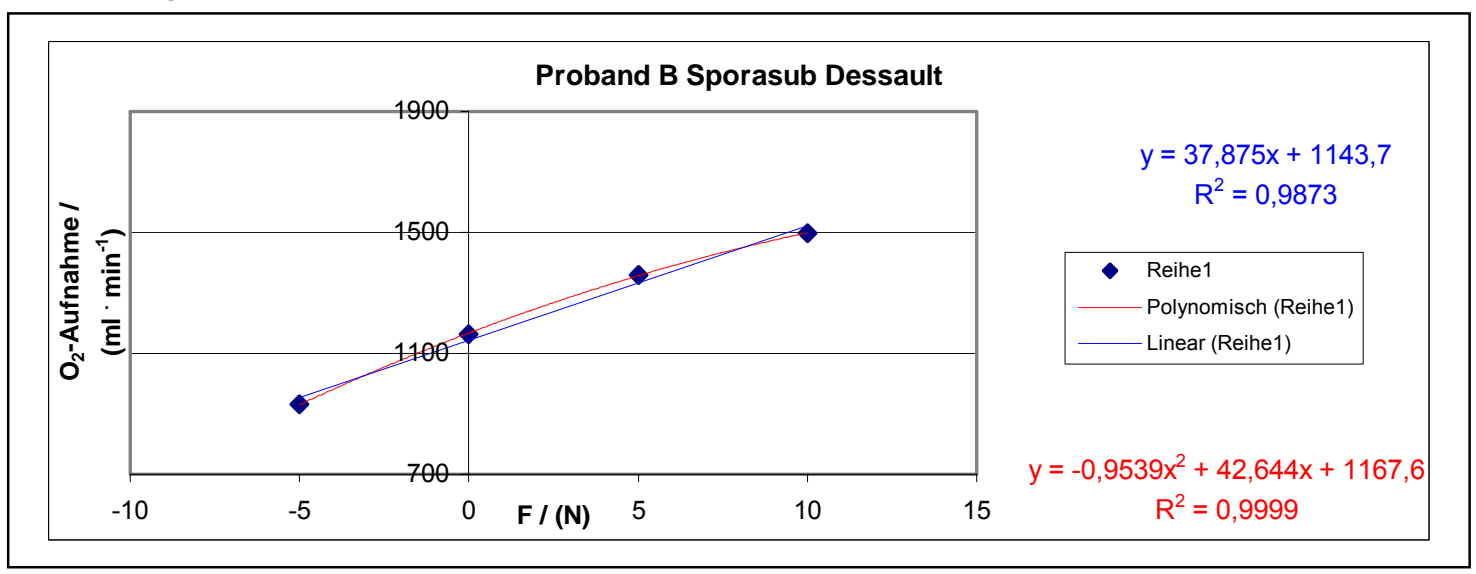

Abbildung 41: Gesamtumsatz Proband B Sporasub Dessault.

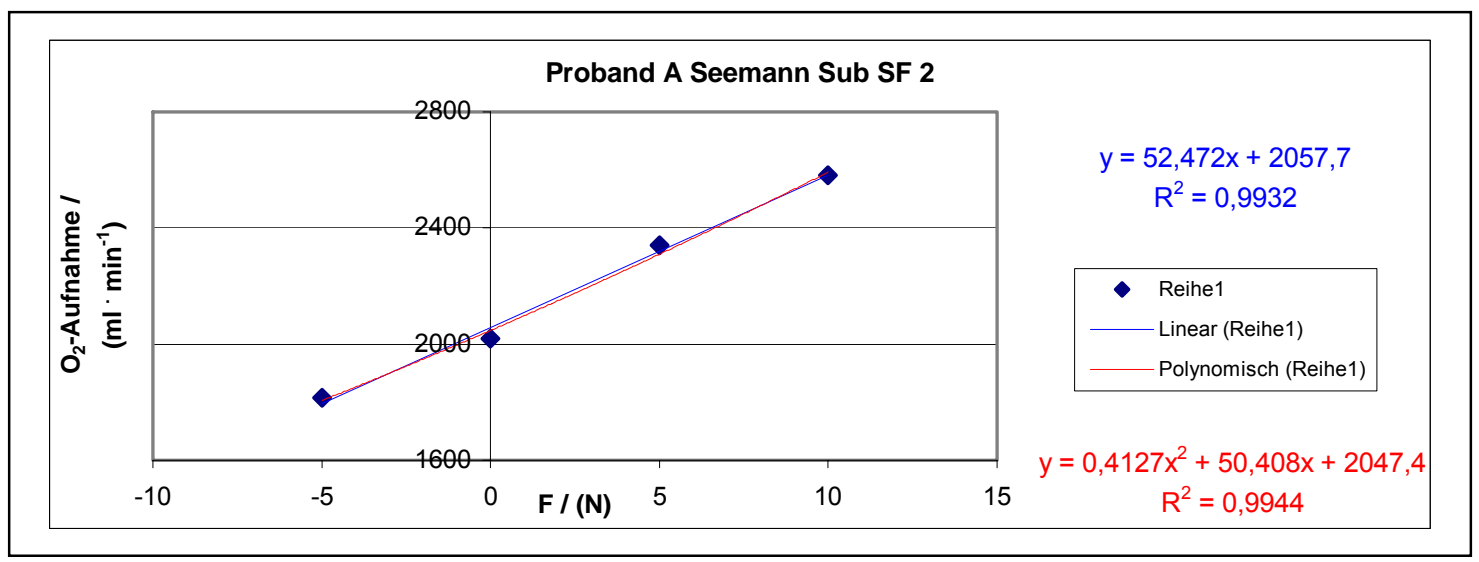

Abbildung 42: Gesamtumsatz Proband A Seemann Sub SF 2. 


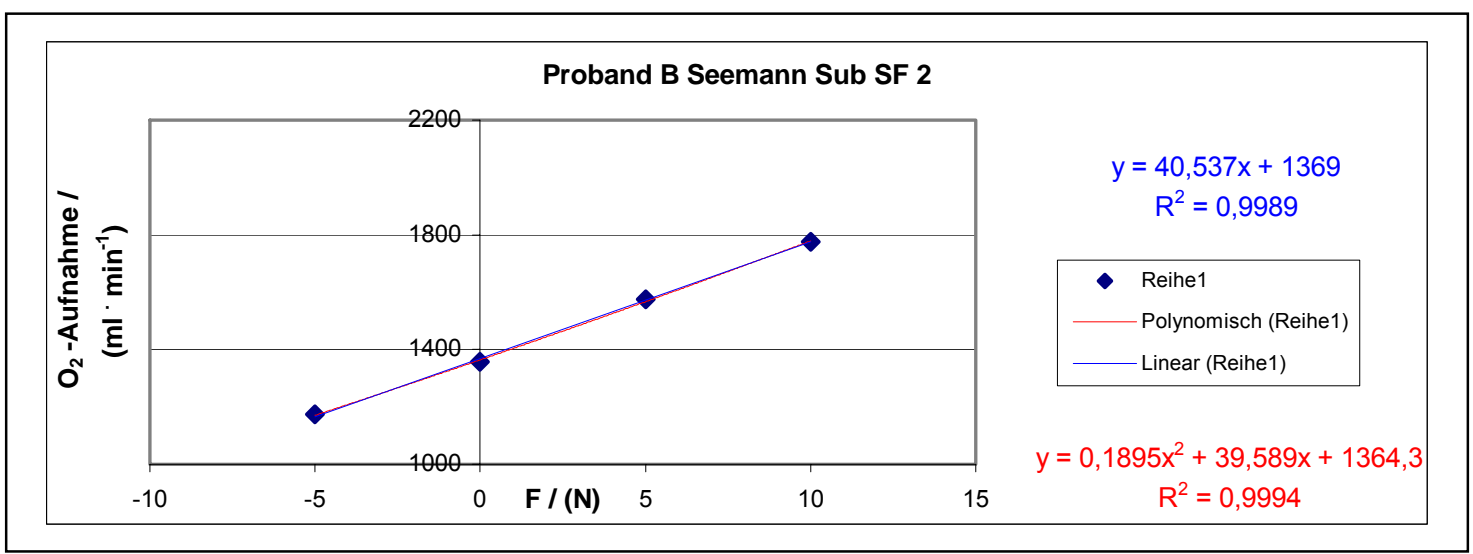

Abbildung 43: $\quad$ Gesamtumsatz Proband B Seemann Sub SF 2.

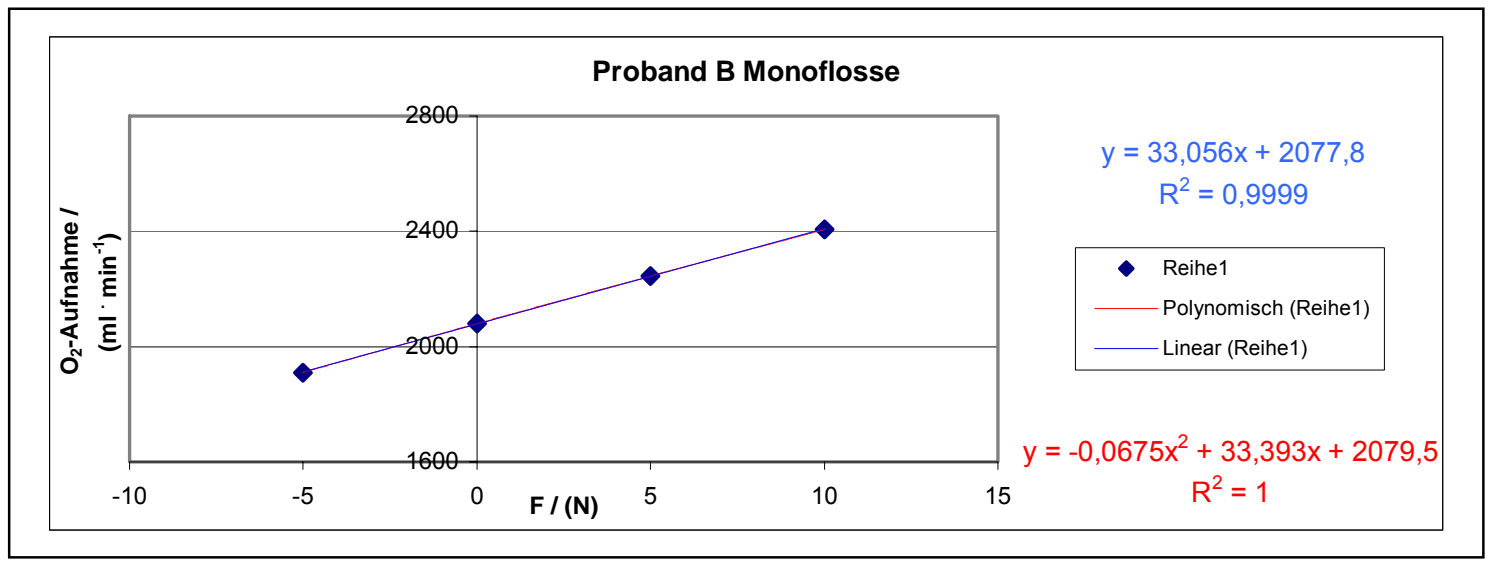

Abbildung 44: Gesamtumsatz Proband B Monoflosse.

Über die Standardabweichung und das Konfidenzintervall kann die Aussagekraft der jeweiligen Geraden beurteilt werden.

\begin{tabular}{|c|c|c|c|c|c|c|c|c|c|}
\hline Proband A & & $\begin{array}{c}\text { Standard- } \\
\text { abweichung }\end{array}$ & $\begin{array}{c}\text { Konfidenz- } \\
\text { intervall }\end{array}$ & $\begin{array}{l}\text { Anzahl } \\
\text { Werte }\end{array}$ & Proband B & & $\begin{array}{c}\text { Standard- } \\
\text { abweichung }\end{array}$ & $\begin{array}{c}\text { Konfidenz- } \\
\text { intervall }\end{array}$ & $\begin{array}{l}\text { Anzahl } \\
\text { Werte }\end{array}$ \\
\hline \multirow[t]{3}{*}{ Mares Volo } & -5 & 166,45 & 145,89 & 5 & Mares Volo & -5 & 84,24 & 52,21 & 10 \\
\hline & 0 & 69,55 & 55,65 & 6 & & 0 & 291,36 & 190,35 & 9 \\
\hline & $\begin{array}{r}5 \\
10\end{array}$ & 105,15 & 84,13 & 6 & & $\begin{array}{r}5 \\
10\end{array}$ & 181,40 & 145,15 & \\
\hline Sporasub & -5 & 146,15 & 101,27 & 8 & Sporasub & $\mid-5$ & 87,63 & 76,81 & 5 \\
\hline \multirow{3}{*}{ Dessault } & 0 & 58,88 & 47,11 & 6 & Dessault & 0 & 278,14 & 181,71 & 9 \\
\hline & 5 & 116,12 & 75,87 & 9 & & 5 & 6,02 & 5,28 & 5 \\
\hline & 10 & 175,67 & 198,78 & 3 & & 10 & 127,35 & 94,34 & 7 \\
\hline Seemann & -5 & 139,77 & 103,54 & 7 & Seemann & $\mid-5$ & 187,66 & 164,48 & 5 \\
\hline \multirow[t]{7}{*}{ Sub SF 2} & 0 & 74,11 & 59,30 & 6 & Sub SF 2 & 0 & 102,01 & 63,23 & 8 \\
\hline & 5 & 116,85 & 86,56 & 7 & & 5 & 106,08 & 69,77 & 8 \\
\hline & 10 & 210,57 & 155,99 & 7 & & 10 & 183,67 & 136,06 & 6 \\
\hline & & & & & Monoflosse & -5 & 172,72 & 138,21 & 6 \\
\hline & & & & & & 0 & 44,90 & 35,92 & 6 \\
\hline & & & & & & 5 & 76,34 & 74,81 & 4 \\
\hline & & & & & & 10 & 447,31 & 309,97 & 8 \\
\hline
\end{tabular}

Tabelle 16: $\quad$ Standardabweichung, Konfidenzintervall und Anzahl der in die Auswertung eingeflossenen Werte für beide Probanden. Auf die rot markierten Daten wird nachfolgend genauer Bezug genommen. 
Mit einer Ausnahme sind mindestens 4 Werte in die Auswertung aufgenommen worden. Im Versuch Proband A Sporasub Dessault $+10 \mathrm{~N}$ sind die Schwankungen sehr stark und keine Einheitlichkeit in den gemessenen Werten festzustellen. Selbst nach der Beschränkung auf 3 Messwerte ist das Konfidenzintervall das mit Abstand Höchste bei Proband A. Alle anderen Werte verteilen sich zwischen 47,11 N und 155,99 N. Bei Proband B ist das Bild einheitlicher. Im Mittel kommen mehr Werte in die Auswertung, wobei allerdings wegen uneinheitlicher Werte in einem Fall die Messwerte über die kompletten 3 Minuten berücksichtigt werden (Sporasub Dessault $0 \mathrm{~N}$ ). Beim Versuch Mares Volo -5 N können 10 Messwerte mit einem Konfidenzintervall von $52,21 \mathrm{~N}$ bei festgestellt werden. Sehr uneinheitlich ist das Bild mit einem Konfidenzintervall von 309,97 mit 8 Messwerten für die Monoflosse bei $+10 \mathrm{~N}$.

\subsubsection{Respiratorischer Quotient}

Der RQ steigt bei allen Probanden an. Dieser Anstieg ist bei Proband A mit der Flosse Sporasub Dessault am ausgeprägtesten. Der $R Q$ steigt von 0,70 über 0,80 und 0,85 bis 0,90 an. Bei der Mares Volo steigt er von 0,84 über 0,91 bis 0,94. Bei der Seemann Sub SF 2 steigt der $R Q$ von 0,84 und stabilisiert sich danach zwischen 0,92 und 0,94 mit leicht abnehmender Tendenz. Der RQ liegt für Proband $B$ mit der Sporasub Dessault annähernd konstant zwischen 0,86 und 0,88. Bei $\pm 0 \mathrm{~N}$ Zusatzkraft erreicht er sein $R Q_{\max }$ mit 0,88 . Mit der Seemann Sub SF 2 ist festzustellen, dass der $R Q$ sich kontinuierlich von 0,77 über 0,81 und 0,83 auf 0,89 ändert. Gleiches gilt für die Mares Volo mit einer Änderung von 0,79 über 0,85 auf 0,89 . Mit der Monoflosse ist bei Proband $B$ ein annähernd linearer Anstieg des $R Q$ bis $+5 \mathrm{~N}$ gegeben. Er stabilisiert sich mit $0,89(+5 N)$ und $0,90(+10 N)$.
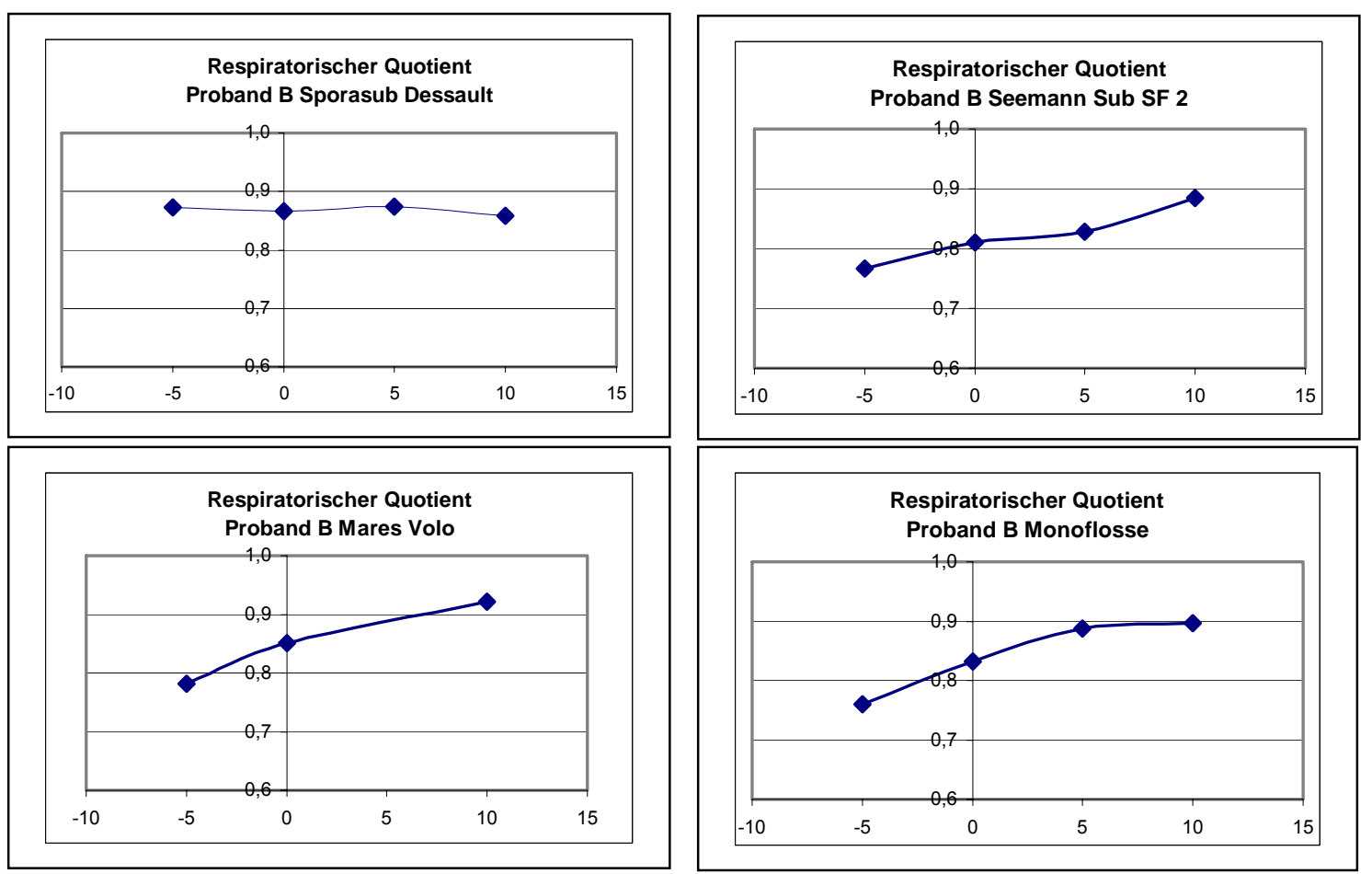

Abbildung 45: $\quad \mathrm{RQ}$ von Proband $\mathrm{B}$ für vier Flossenmodelle. 

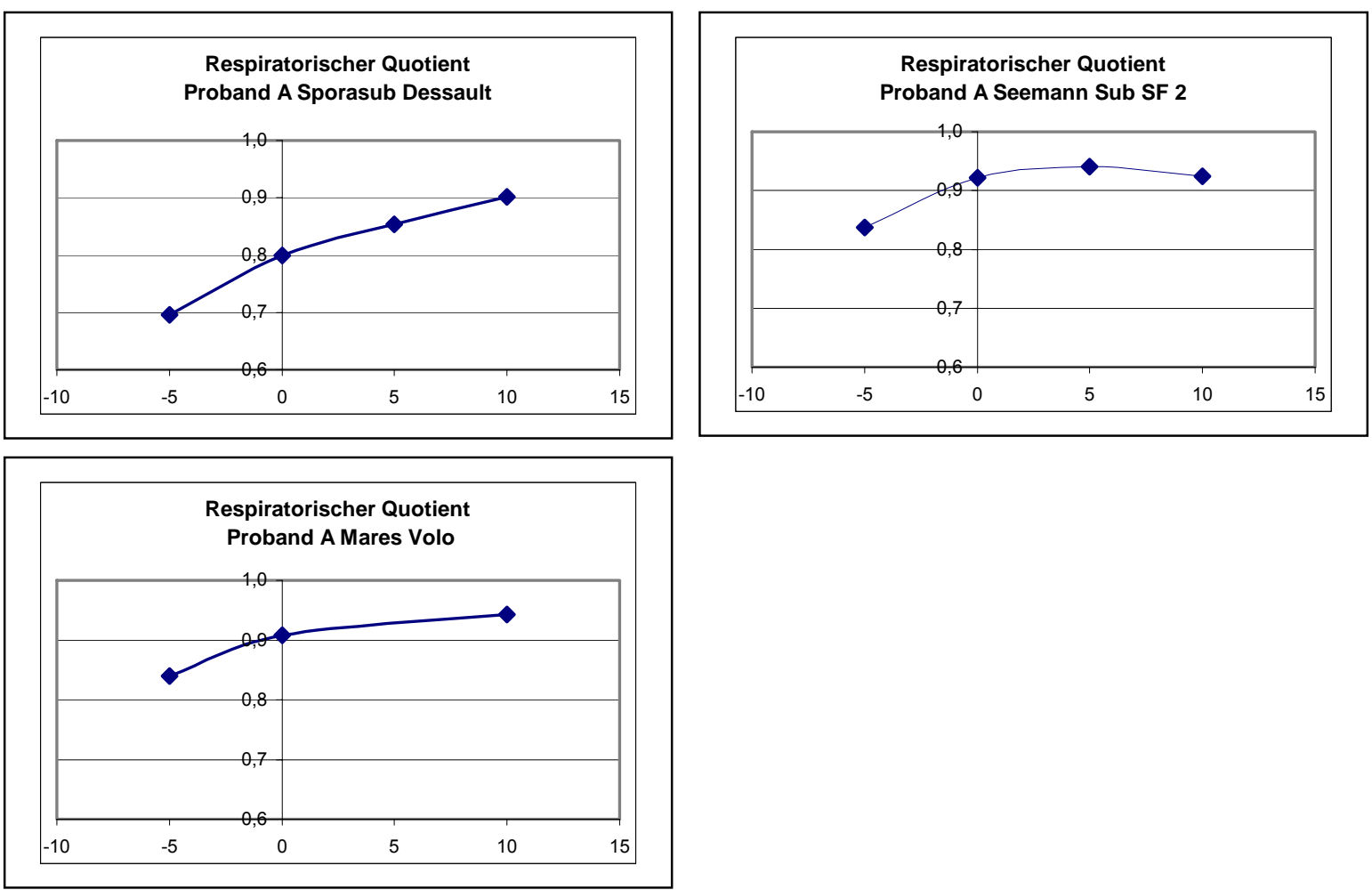

Abbildung 46: $\quad \mathrm{RQ}$ von Proband $\mathrm{A}$ für drei Flossenmodelle.

\subsubsection{Wirkungsgrad}

Die Wirkungsgrade beziehen sich, da die Bestimmtheitsmaße es in allen Versuchen zulassen, auf die lineare Auswertung der Messergebnisse. Von Interesse ist der Bruttowirkungsgrad ebenso wie der Nettowirkungsgrad, der sich auf den Bruttoumsatz abzüglich des Ruheumsatzes bezieht.

\subsubsection{Nettowirkungsgrad}

Die Kurven für die gleichen Flossen verlaufen bei beiden Probanden annähernd parallel. Die Monoflosse hat mit ca. 8,3\% - 8,6\% den höchsten Nettowirkungsgrad. Mit der Sporasub Dessault realisiert Proband B einen $\eta_{\text {netto }}$ zwischen 5,46\% - 5,82\%, während Proband $A$ mit $5,92 \%$ bis $6,35 \%$ mit dieser Flosse einen geringfügig höheren $\eta_{\text {netto }}$ erreicht und sich die Werte bis zur letzten Belastungsstufe annähern. Bei der Seemann Sub SF 2 liegen die Kurven etwa 1,2\% auseinander. Bei Proband B liegt sie mit Werten zwischen 5,20\% und 5,37 \% über der Kurve von Proband A mit 3,97\% $4,12 \%$. Bei der Mares Volo ist der Nettowirkungsgrad bei beiden Probanden fast identisch und liegt einheitlich zwischen 3,64\% und 3,84\%, wobei Proband B zwischen $0,06 \%$ und $0,09 \%$ niedriger als Proband $A$ ist. 

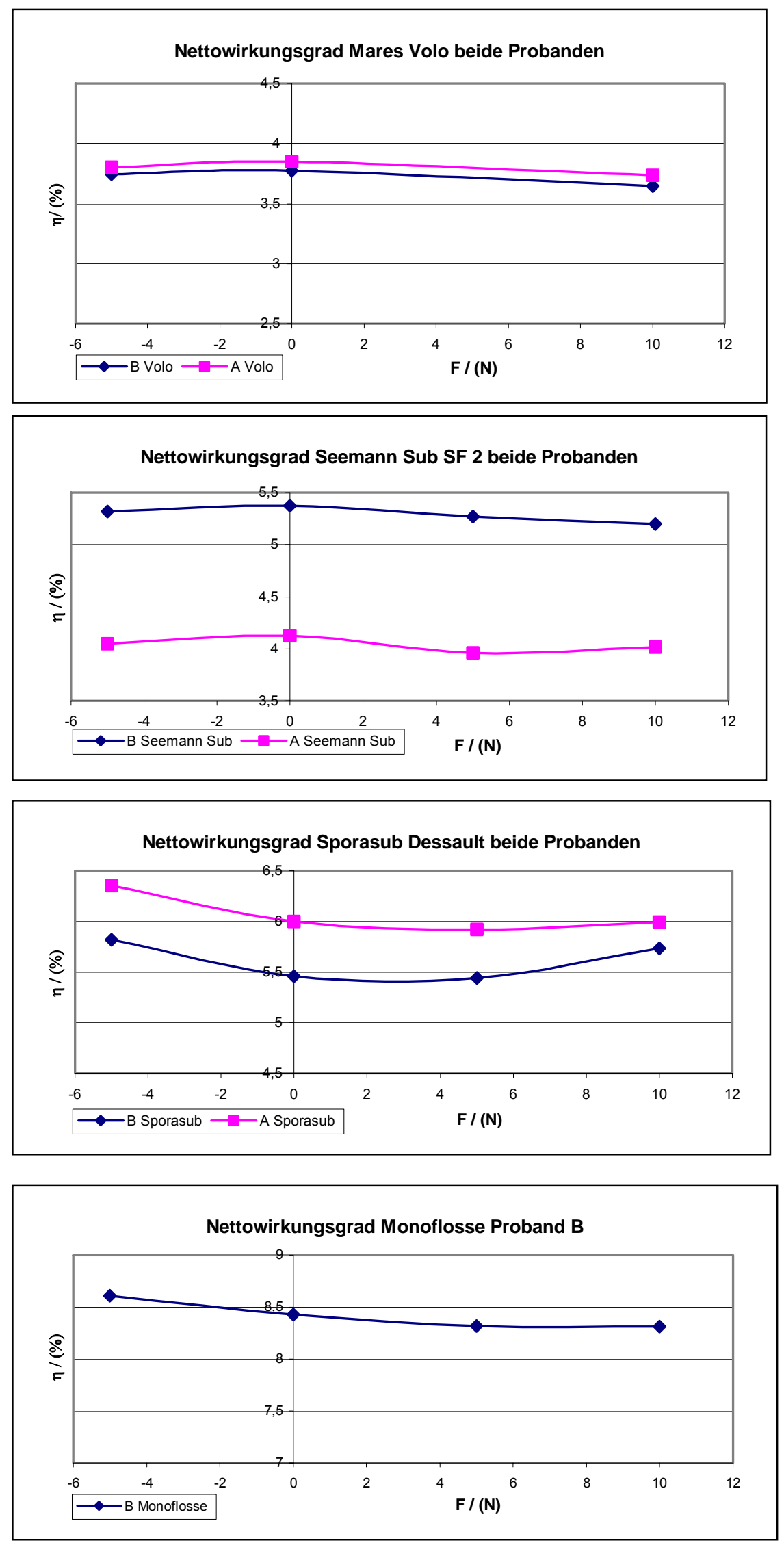

Abbildung 47: Nettowirkungsgrad für beide Probanden mit allen Flossenmodellen. 


\subsubsection{Bruttowirkungsgrad}

Die Verhältnisse beim Bruttowirkungsgrad sind denen beim Nettowirkungsgrad vergleichbar. Die Monoflosse hat mit im Mittel $\eta_{\text {brutto }}=6,8 \%$ den höchsten Bruttowirkungsgrad, wobei dieser fast konstant bleibt. Bei der Sporasub Dessault steigen die Kurven für beide Probanden an. Bei Proband $A$ sind in den ersten drei Belastungsstufen höhere Werte als bei Proband B zu beobachten. Die Werte von Proband B steigen stärker an, so dass in der letzten Belastungsstufe beide Probanden einen fast identischen $\eta_{\text {brutto }}$ von 4,4\% (Proband $A$ ) und 4,34\% (Proband B) erreichen. Bei der Seemann Sub SF 2 liegt der Bruttowirkungsgrad von Proband B deutlich $(0,6 \%$ bis $0,8 \%$ ) über dem von Proband $A$. Die Kurven verlaufen parallel und steigen an. Letzteres gilt auch für die Mares Volo. Mit dieser Flosse erreichen beide Probanden den niedrigsten Wirkungsgrad. Er liegt bei maximal 2,97\% (Proband B) und 3,05\% (Proband $A$ ) in der letzten Belastungsstufe.
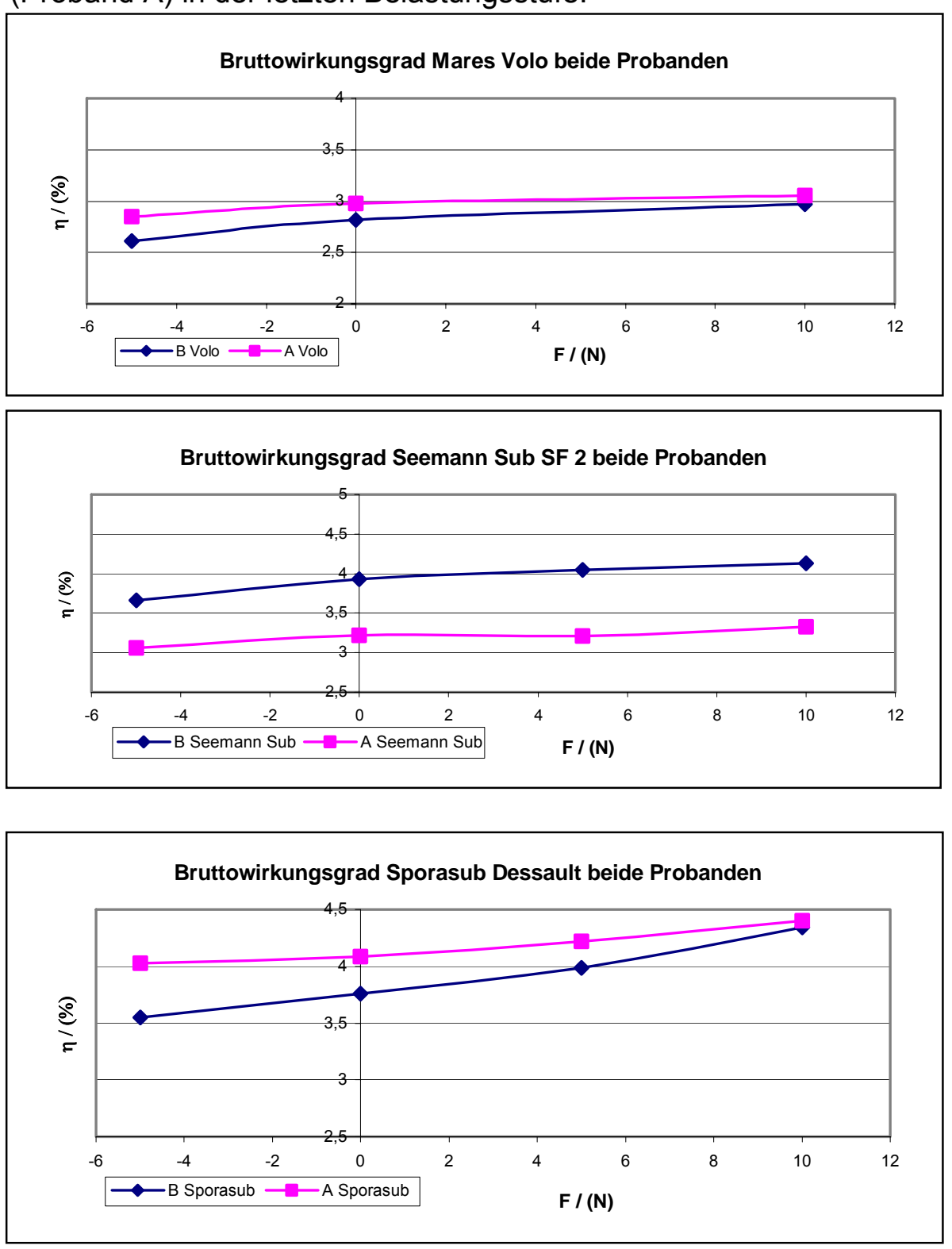

Abbildung 48: Bruttowirkungsgrad für beide Probanden mit den Flossen Mares Volo, Seemann Sub SF 2 und Sporasub Dessault. 


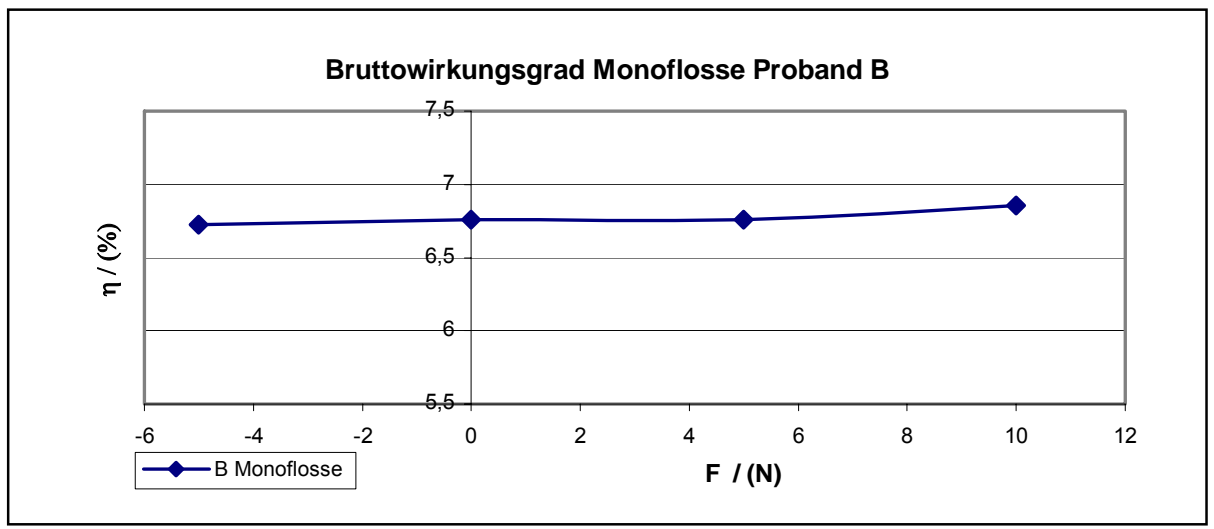

Abbildung 49: $\quad$ Bruttowirkungsgrad für Proband B mit der Monoflosse.

\subsubsection{Mechanische Leistung}

Für Proband B ist die Monoflosse diejenige, bei der die höchste mechanische Leistung erbracht wird, da sie mit der höheren Geschwindigkeit von 0,9426 m $\mathrm{s}^{-1}$ geschwommen wurde. Alle anderen Flossen liegen bei beiden Probanden dicht nebeneinander, wobei für Proband $B$ die Sporasub Dessault etwas günstiger ist als die Mares Volo. Die Seemann Sub SF 2 weist die schlechteren Werte auf. Bei Proband A sind Sporasub Dessault und Seemann Sub SF 2 fast deckungsgleich. Mit der Mares Volo muss die höchste Leistung erbracht werden.

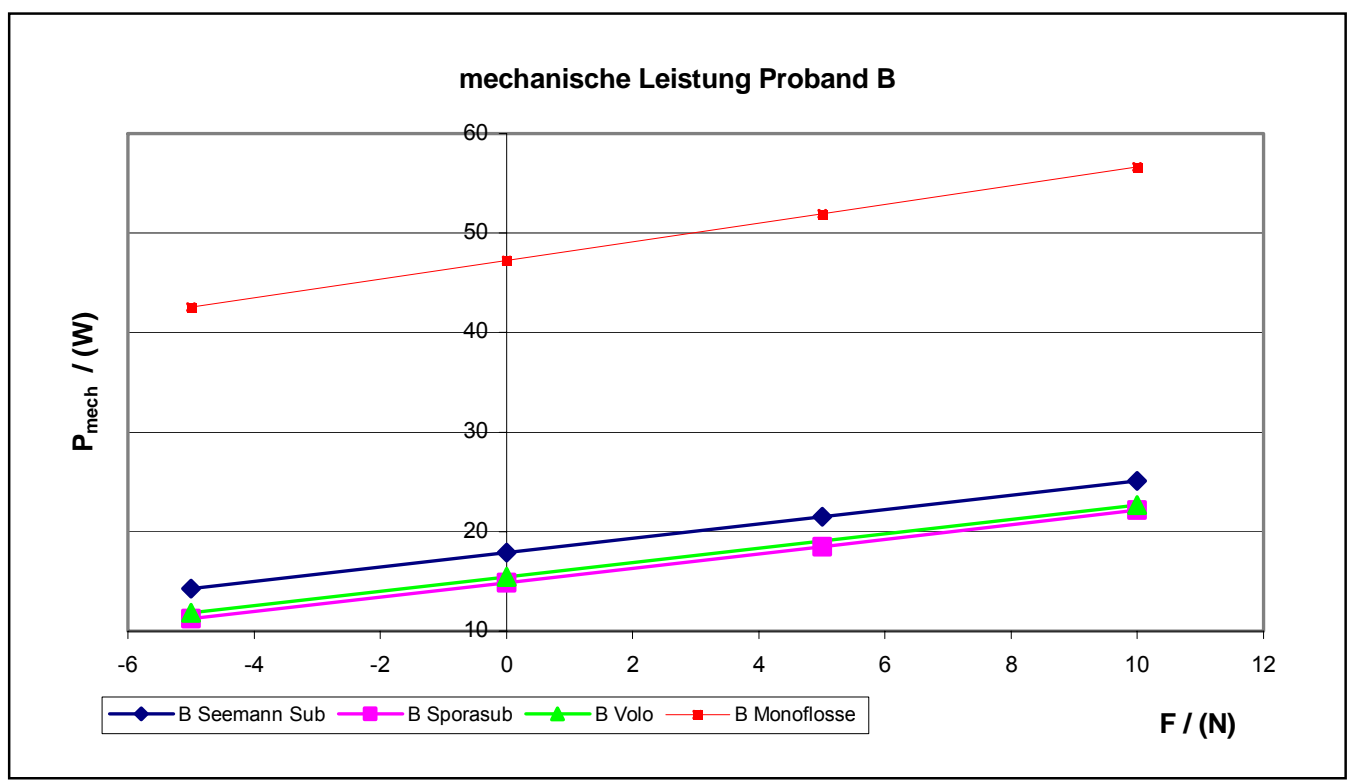

Abbildung 50: mechanische Leistung Proband B mit vier Flossen. 


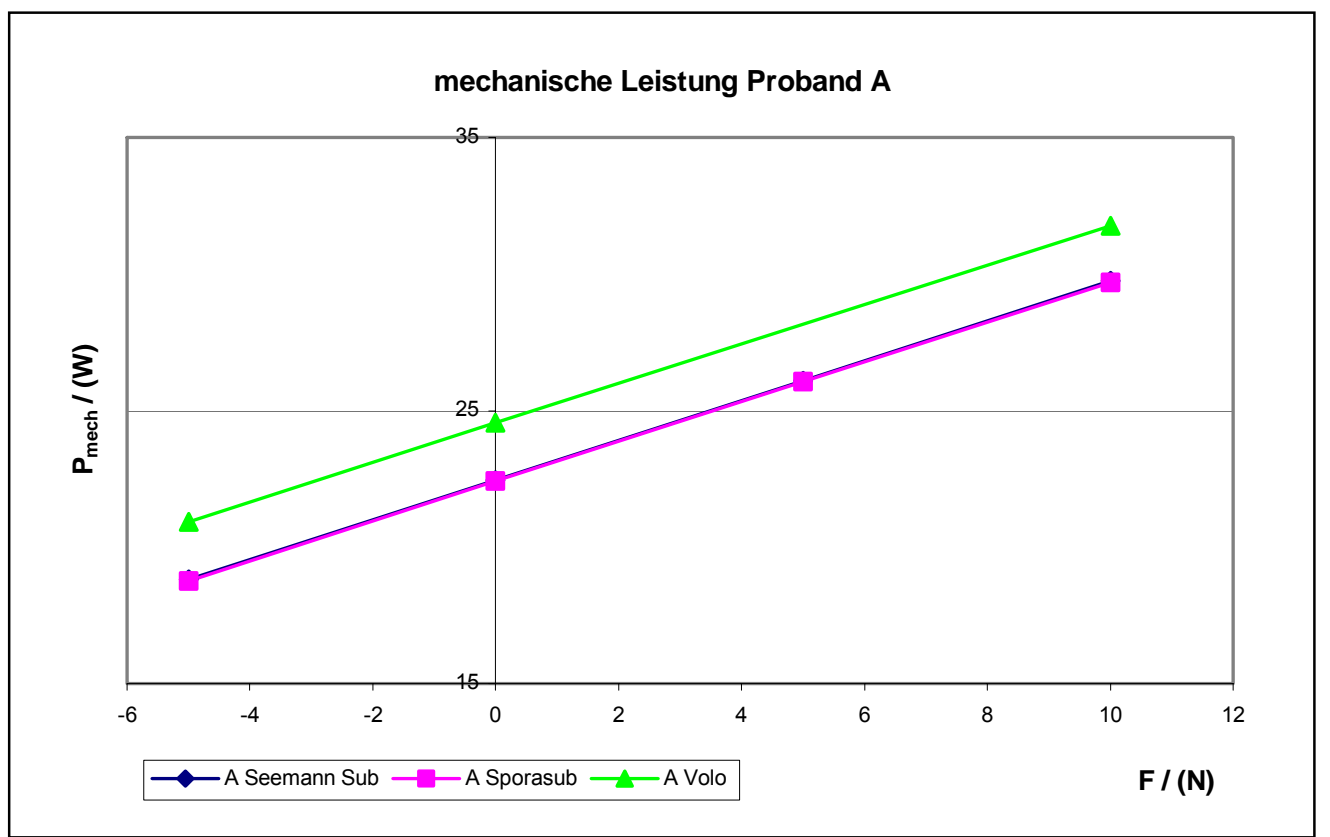

Abbildung 51: mechanische Leistung Proband A mit drei Flossen.

\subsubsection{Dynamischer Widerstand}

Ziel dieser Auswertung ist, wie in Kapitel 3.3.4 dargestellt, über die Extrapolation der Arbeitssauerstoffaufnahme auf die Abszisse des Widerstandes den aktiven Widerstand zu bestimmen. Dazu sollen im Folgenden zur Vereinfachung und besseren Praktikabilität die Mittelwerte der Arbeitssauerstoffaufnahme auf den Belastungsstufen benutzt werden, sofern diese Vorgehensweise begründbar ist und Ergebnisse liefert, die diese rechtfertigen.

Da in allen Fällen das Bestimmtheitsmaß höher ist als 0,95 (siehe Kap. 5.5.2), ist es nicht geboten, den Arbeitsumsatz als 1. Ableitung von $f(0) \mathrm{zu}$ berechnen. Er wird deshalb in den folgenden Abbildungen als lineare Funktion dargestellt.

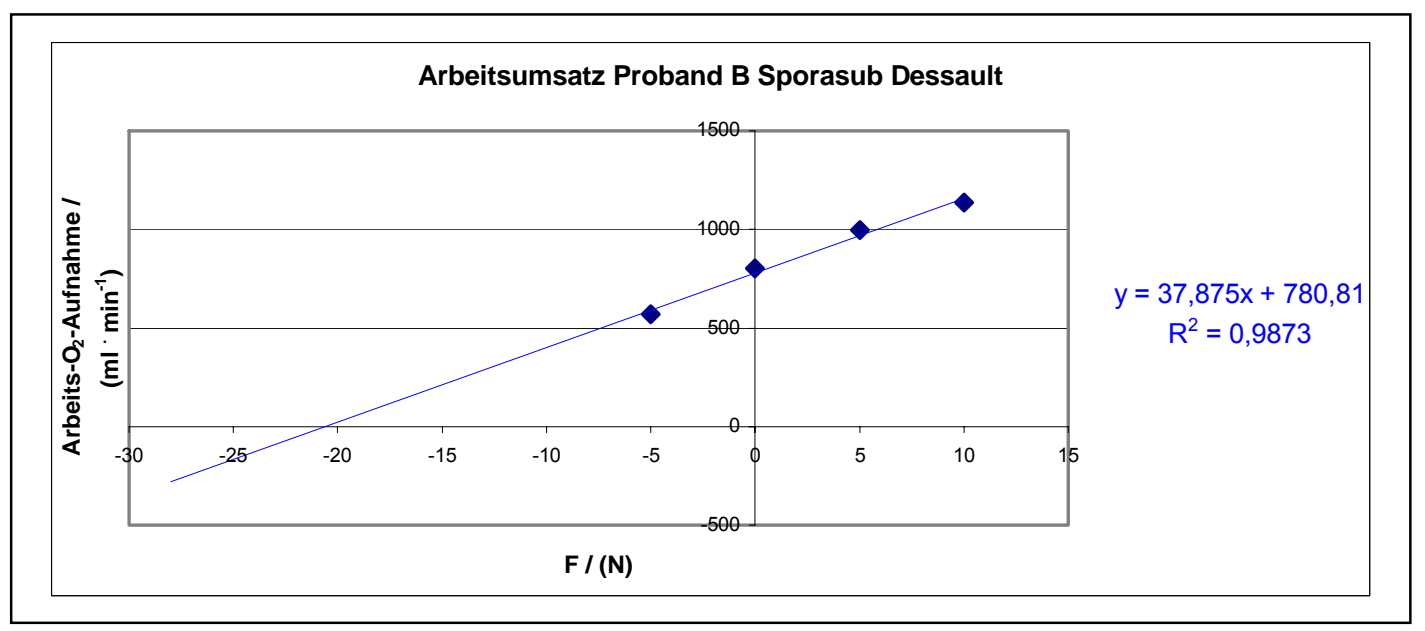

Abbildung 52: Extrapolation des Arbeitsumsatzes Proband B Sporasub Dessault. 


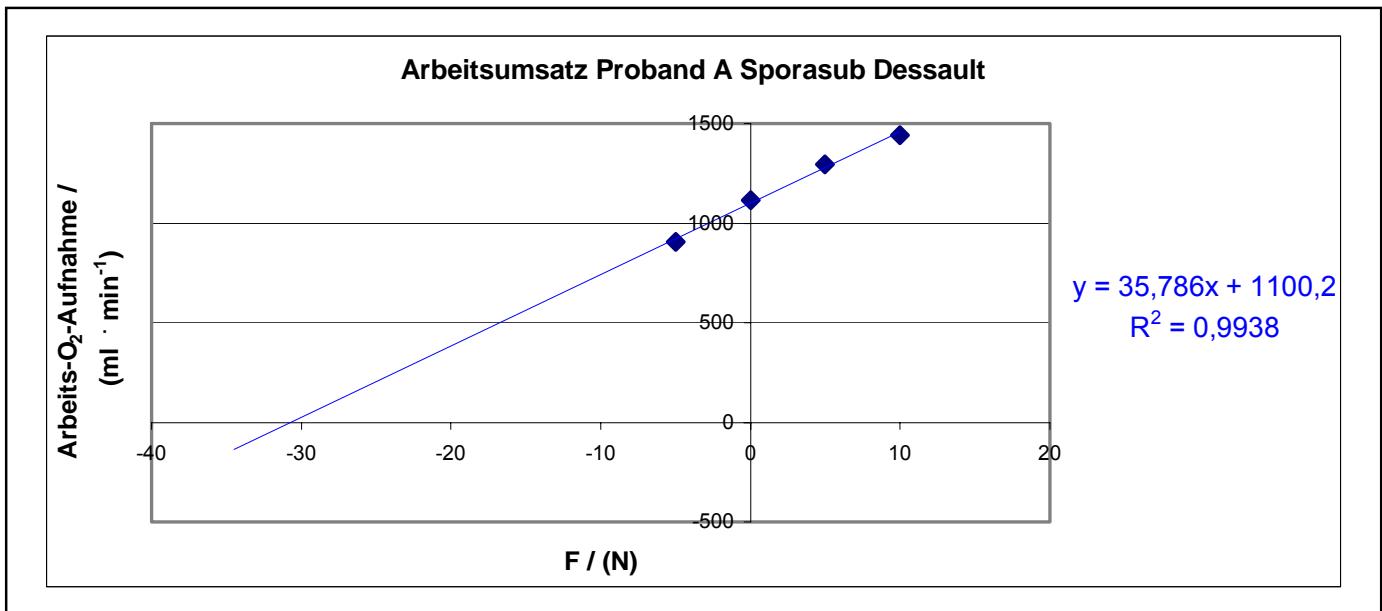

Abbildung 53: Extrapolation des Arbeitsumsatzes Proband A Sporasub Dessault.

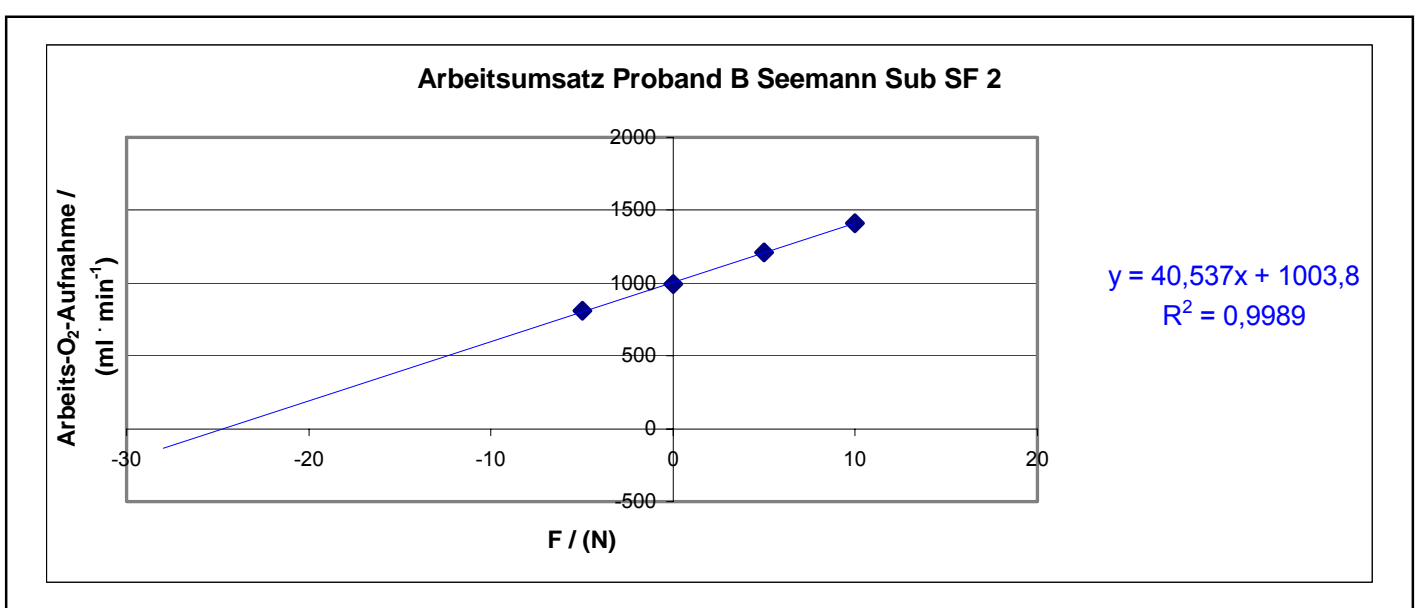

Abbildung 54: Extrapolation des Arbeitsumsatzes Proband B Seemann Sub SF 2.

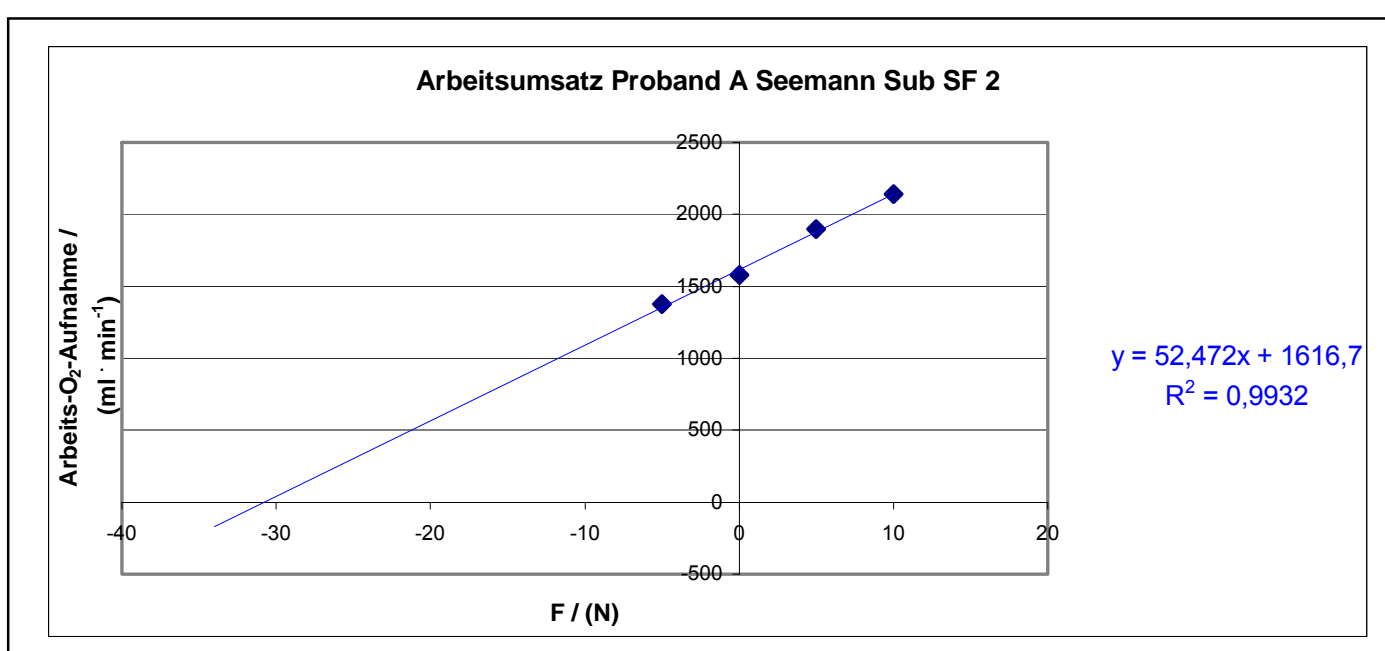

Abbildung 55: Extrapolation des Arbeitsumsatzes Proband A Seemann Sub SF 2. 


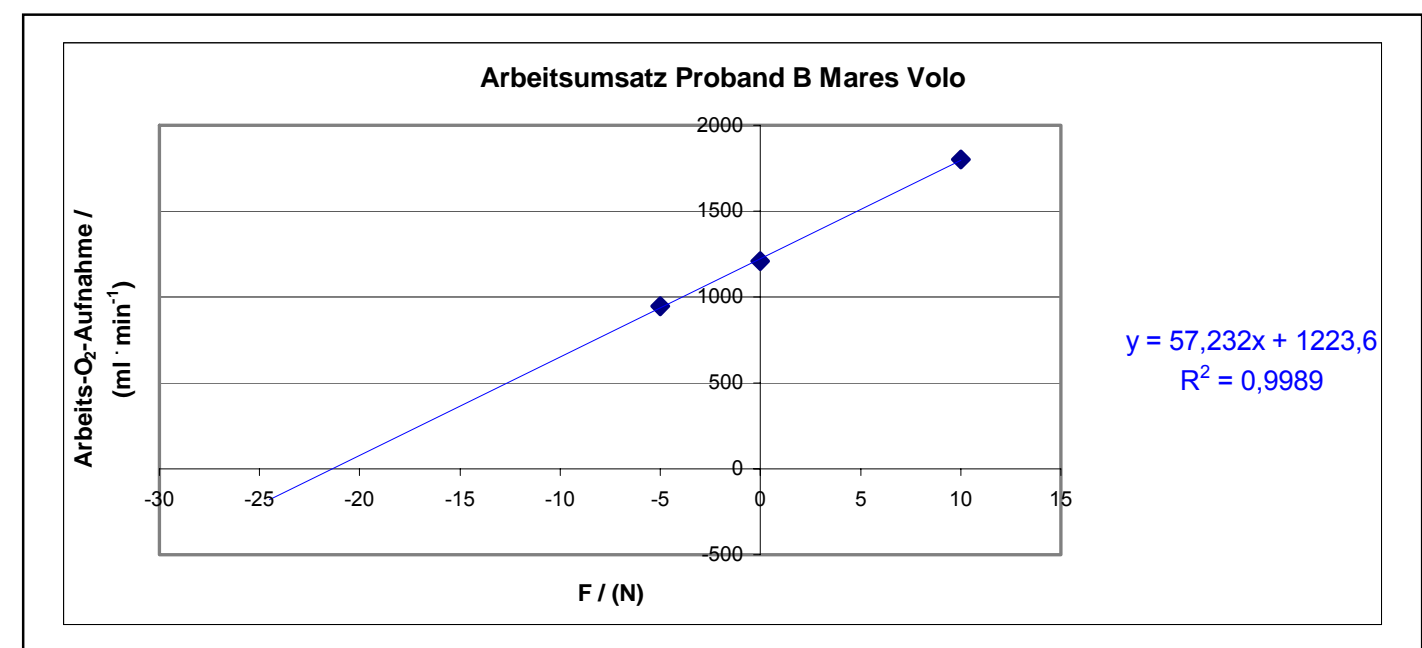

Abbildung 56: Extrapolation des Arbeitsumsatzes Proband B Mares Volo.

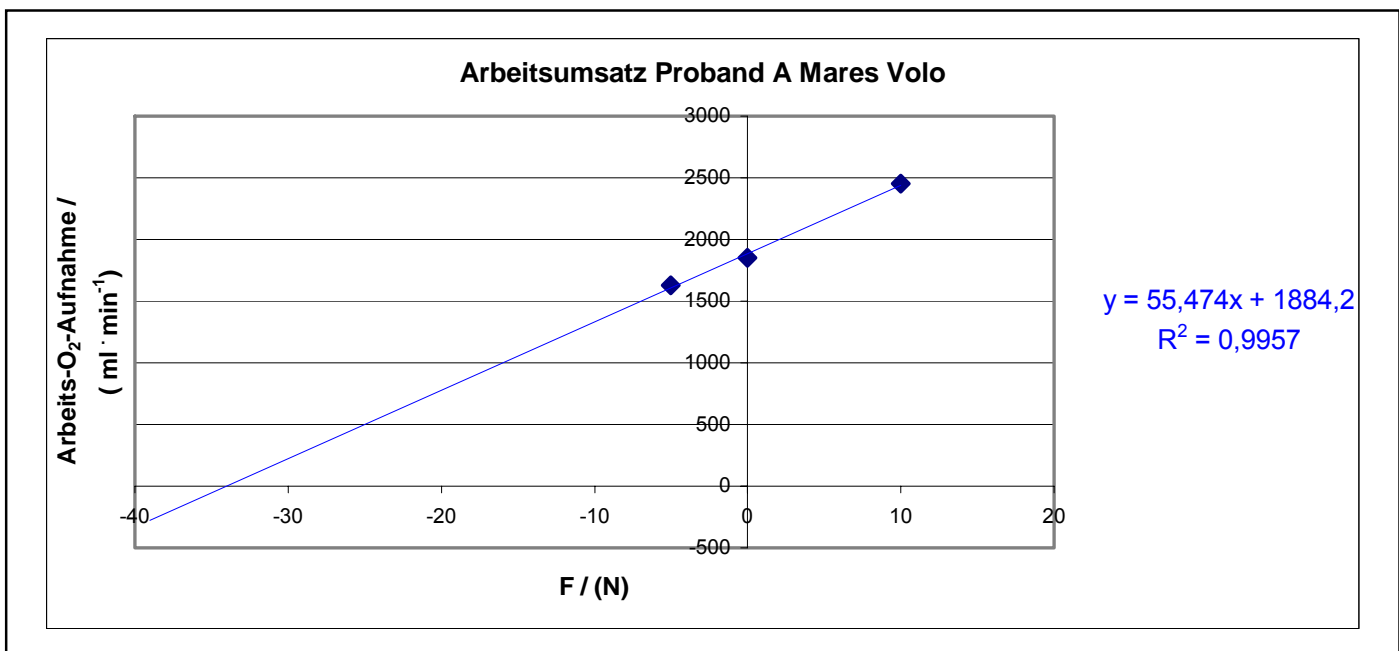

Abbildung 57: Extrapolation des Arbeitsumsatzes Proband A Mares Volo.

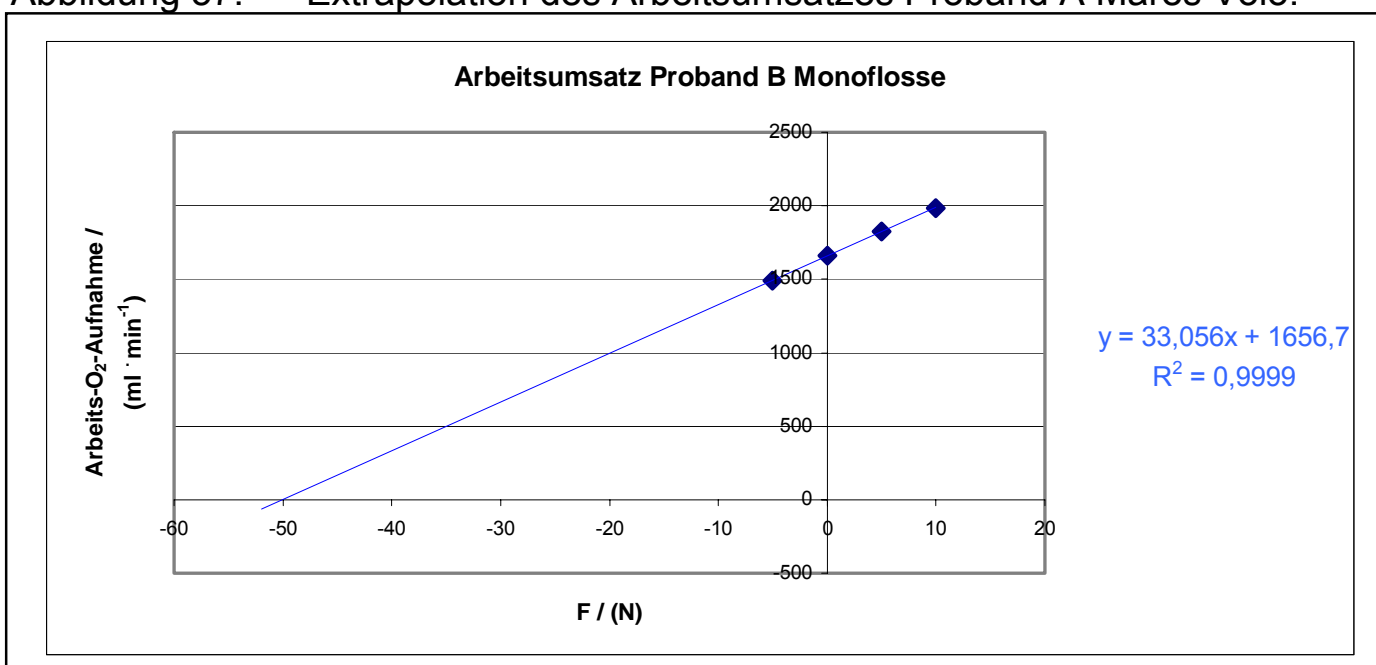

Abbildung 58: Extrapolation des Arbeitsumsatzes Proband B Monoflosse. 


\subsubsection{Vergleich der passiven und dynamischen Widerstandskraft}

Im Folgenden werden die durch die Extrapolation auf der Abszisse dargestellten Kraftwerte aller ausgewählten Werte und der Mittelwerte für die einzelnen Flossentypen gegenübergestellt.

\begin{tabular}{|l|c|c|c|}
\hline Proband /Flosse & Geschwindigkeit & $\mathrm{F}_{\mathrm{Dp}}$ & $\mathrm{F}_{\mathrm{Dd}}$ \\
\hline & $\left(\mathrm{m} \cdot \mathrm{s}^{-1}\right)$ & $(\mathrm{N})$ & $(\mathrm{N})$ \\
\hline A / Mares Volo & 0,7288 & 27,68 & 33,97 \\
\hline A / Seemann Sub SF 2 & 0,7288 & 30,46 & 30,81 \\
\hline A / Sporasub Dessault & 0,7288 & 25,95 & 30,74 \\
\hline B / Mares Volo & 0,7288 & 33,81 & 21,38 \\
\hline B / Seemann Sub SF 2 & 0,7288 & 22,33 & 24,76 \\
\hline B / Sporasub Dessault & 0,7288 & 20,84 & 20,62 \\
\hline B / Monoflosse & 0,9426 & 39,56 & 50,12 \\
\hline
\end{tabular}

Tabelle 17: $\quad$ Gegenüberstellung von $F_{D p}$ und $F_{D d}$.

In den Versuchen A / Mares Volo, A / Sporasub Dessault, B / Seemann Sub SF 2 und $B$ / Monoflosse übersteigt der Wert von $F_{D d}$ den Wert von $F_{D p}$ deutlich, zwischen 2,43 N und 10,56 N. Die Werte von $F_{D d}$ und $F_{D p}$ sind in den Versuchen A / Seemann Sub SF 2 und $B$ / Sporasub Dessault fast identisch. Die Differenz beträgt 0,35 N und -0,22 N. Im Versuch $B$ / Mares Volo ist $F_{D d}$ um 12,43 N niedriger als $F_{D p}$. Die Werte für den Versuch $\mathrm{B} /$ Mares Volo $\mathrm{F}_{\mathrm{Dp}}$ werden nicht berücksichtigt, da unter 5.4 bereits auf die Besonderheiten in der Körperhaltung von Proband $B$ hingewiesen wurde, welche im Ergebnis zu einem deutlich über $F_{D d}$ liegendem Kraftwert $F_{D p}$ führen, welcher nicht plausibel ist.

\subsubsection{MET}

Die Leistung kann auch relativ zum Körpergewicht und damit in MET angegeben werden. Dazu muss $\dot{V} \mathrm{O}_{2 \max }$ zur Körpermasse ins Verhältnis gesetzt werden. Zu beachten ist, dass alle Flossen bei $0,8 \mathrm{~m} \cdot \mathrm{s}^{-1}$ (real $0,7288 \mathrm{~m} \cdot \mathrm{s}^{-1}$ ), die Monoflosse von Proband B bei $1,0 \mathrm{~m} \cdot \mathrm{s}^{-1}$ (real $0,9426 \mathrm{~m} \cdot \mathrm{s}^{-1}$ ) geschwommen wurde. Der Grenzwert von $45 \mathrm{ml} \mathrm{O}_{2} \cdot \mathrm{kg}^{-1} \cdot \mathrm{min}^{-1}$ genannt für Berufstaucher als Ausschlusskriterium für die Tauchtauglichkeit wird von keinem der Probanden erreicht. 
Bei beiden Probanden ist die gleiche Reihenfolge der Flossen gegeben. Beide Probanden benötigen in Relation zum Körpergewicht mit der Sporasub Dessault den geringsten $\dot{V} \mathrm{O}_{2}$ pro Minute. Bei beiden Probanden folgt die Seemann Sub SF 2 und als letzte die Mares Volo. Mit der Monoflosse erreicht Proband B seine höchsten Werte, während Proband A mit der Mares Volo seine höchsten Werte erreicht. Bemerkenswert ist, das bei $+10 \mathrm{~N}$ von Proband B mit dieser Flosse der höchste erreichte Wert realisiert wird. Dieser liegt höher als der, der von Proband B bei höherer Geschwindigkeit mit der Monoflosse erreicht wird.

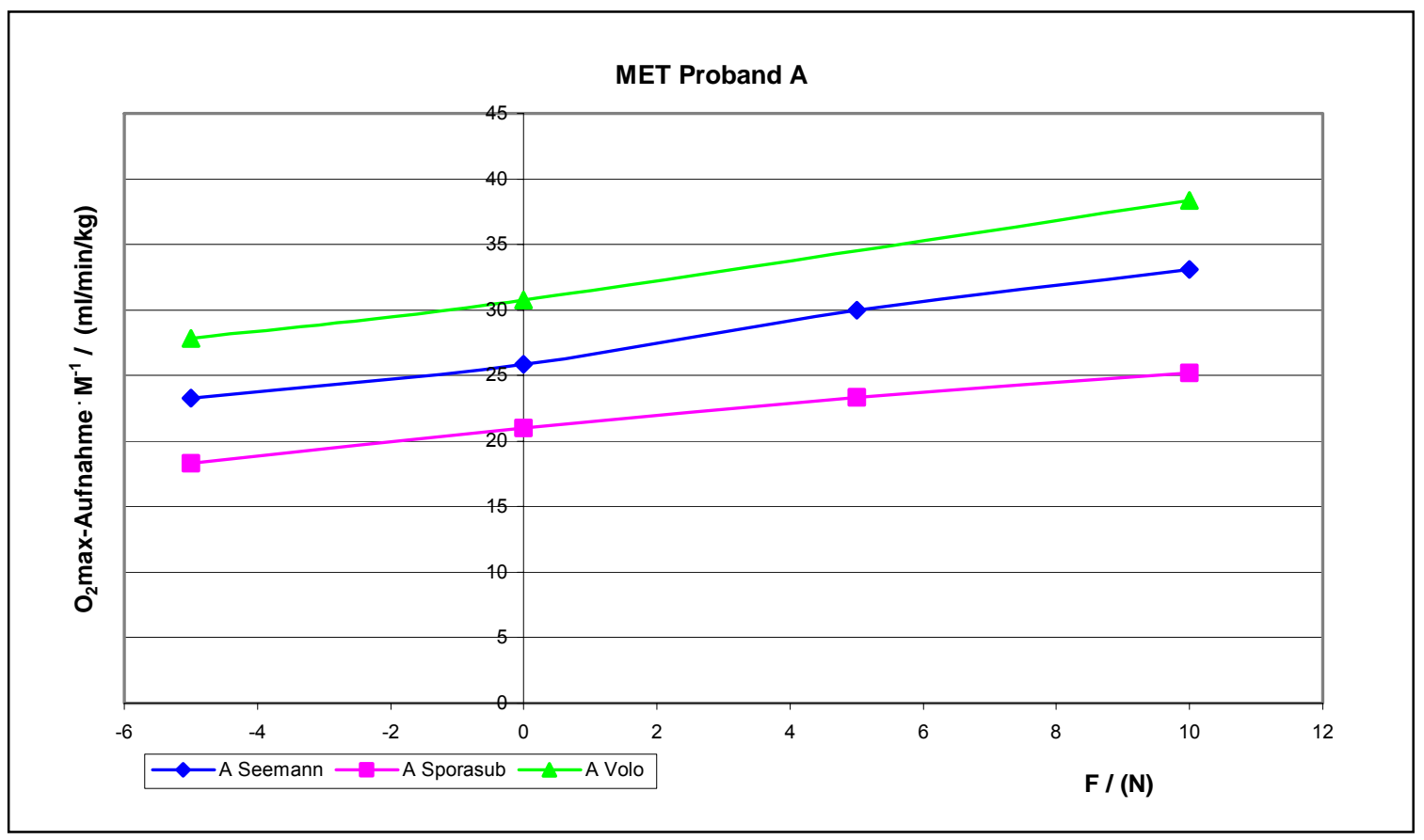

Abbildung 59: MET Proband A drei Flossen.

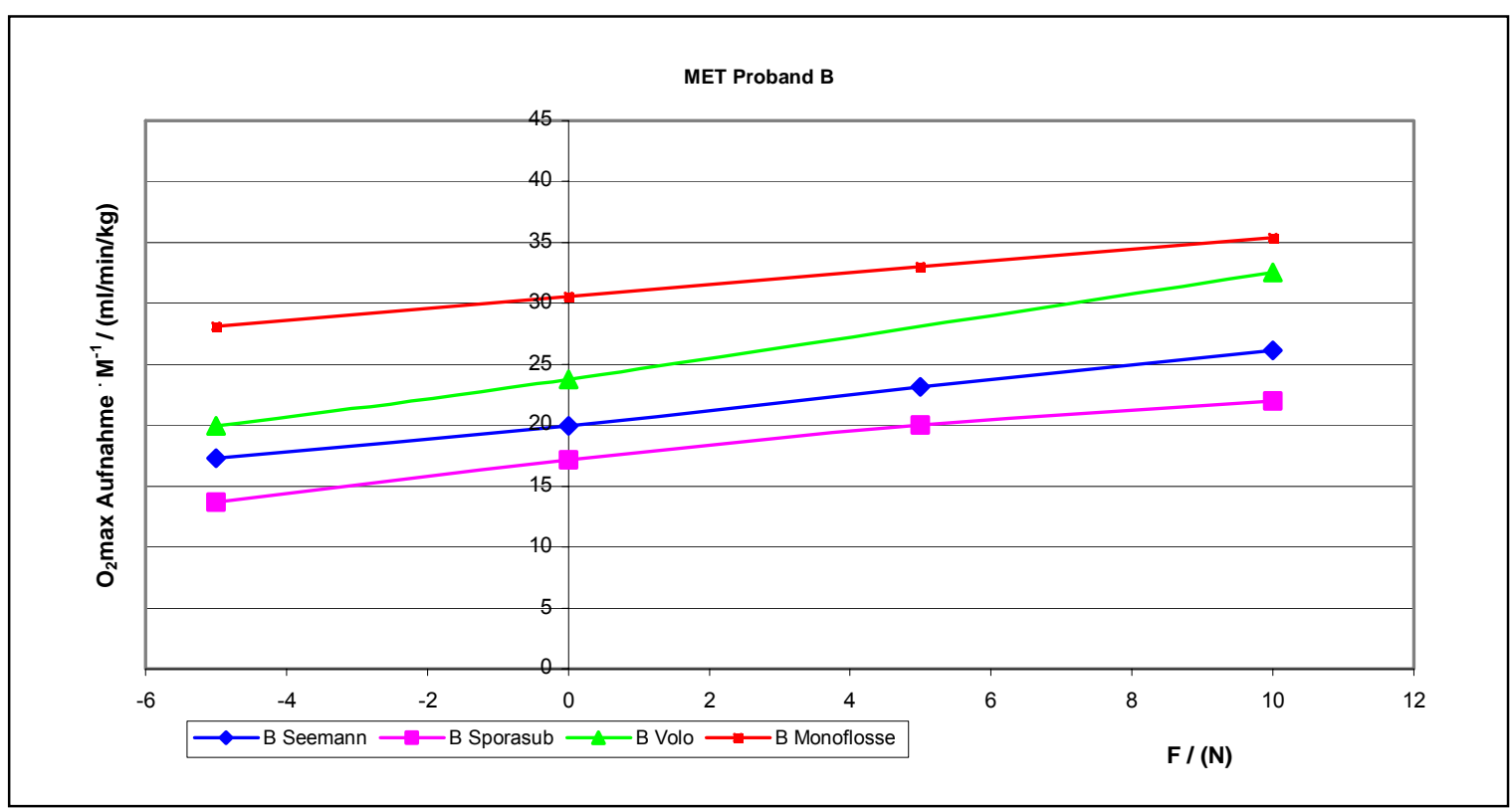

Abbildung 60: MET Proband B vier Flossen. 


\subsection{Diskussion der Methode}

\subsubsection{Bestimmung der Strömungsverhältnisse im Kanal (Messreihe 1) und die Kalibrierung des Kraftaufnehmers}

Die Messung von Geschwindigkeiten mit Impellern ist ein in der Physik, neben der Staudruckmethode, gut eingeführtes Verfahren, welches keine Probleme aufwarf, ebenso wenig wie die Kalibrierung des Kraftaufnehmers.

\subsubsection{Bestimmung der Kraftwerte für unterschiedliche Auftriebskörper (Messreihe 2)}

Die Messungen ergaben eindeutige, durch statistische Kenndaten als sicher geltende Ergebnisse (siehe Anhang 4 und 5).

Problematisch war der Versuchaufbau hinsichtlich der beiden niedrigen Strömungsgeschwindigkeiten. Hier konnte es dazu kommen, dass die Probanden eine Gleitlage nicht über die gesamte Zeitdauer halten konnten und die Flossen zeitweise den Beckenboden berührten. Dadurch erhöht sich der Stirnwiderstand und als Konsequenz ergeben sich für drei Schleppversuche in den ersten beiden Geschwindigkeiten geringfügig oberhalb der polynomischen Funktion liegende Kraftwerte (Abb. 17-19).

Herabhängende Beine resp. Flossen kommen nur selten, jedoch vor allem bei ungeübten Tauchern vor. Grundsätzlich lernt ein Taucher, sich im Zustand der Schwerelosigkeit so auszubalancieren, dass er horizontal im Wasser liegt. Im Versuchaufbau wird die Kraft an den Händen der Probanden aufgenommen. Da aber die Kraft jetzt an den Händen angreift, ist der Proband nicht mehr in der Lage, sich auszubalancieren und ist auf die Hilfe der Strömung angewiesen, um in eine optimale Gleitlage zu kommen. Durch den langen Hebel kann es in Verbindung mit einer geringen Geschwindigkeit zu der beschriebenen Lageveränderung kommen. Dadurch bestehen geringe Unterschiede $\mathrm{zu}$ der realen Situation des Tauchers für den genannten Bereich. In den oberen drei Geschwindigkeitsstufen bestand das Problem nicht.

Auf Grund der Probandenzahl ist eine statistische Bewertung der Ergebnisse weder möglich noch vorgesehen. Aber schon die Gleichheit bzw. Ähnlichkeit der Kurvenverläufe in den Versuchen der Probanden bei gleichem Auftriebskörper lässt vermuten, dass es sich bei der vorgestellten Methode um ein geeignetes Vorgehen handelt, aussagekräftige Kraftwerte zu ermitteln. Unterschiede im Vergleich beider Probanden treten nur bei den Kraftverläufen bei der Kragenweste auf. Dieser Unterschied scheint darin begründet zu sein, dass Proband B die Weste sehr eng trug. 
Er hat als erster von beiden Probanden diesen Versuch absolviert. Der Schrittgurt war sehr kurz geschnallt, so dass er inn im Schritt beeinträchtigte. Nach dem Versuch klagte er darüber, dass inm der Schrittgurt der Kragenweste Schmerzen bereitet habe. Dadurch lag die Kragenweste und speziell die Westenflasche aber extrem eng am Körper an und führte so zu geringen Kraftwerten. Bei Proband A wurde darauf geachtet, dass der Schrittgurt der Kragenweste korrekt angelegt wurde und ihm genug Bewegungsfreiheit lässt. Dadurch hängt die Westenflasche aber weiter herunter, es kommt zu einer größeren Anstromfläche $A$ und damit zu höheren Widerstandswerten $F_{w}$. Die während der Versuche aufgenommenen Videos bestätigen dieses.

\subsubsection{Bestimmung der Kraftwerte für unterschiedliche Flossen (Messreihe 3)}

Die Messungen ergaben eindeutige, durch statistische Kenndaten als sicher geltende Ergebnisse.

Der Einfluss der Körperhaltung ist im Vergleich mit Messreihe 2 größer, da die Probanden mit weniger Masse geschleppt werden. Es traten ähnliche Probleme in den ersten beiden Geschwindigkeitsstufen auf. Zusätzlich hatte eine Besonderheit in der Körperhaltung unerwünschten Einfluss auf die Messergebnisse. Beide Probanden haben in jeweils einem Versuch die Unterschenkel im Kniegelenk zeitweise bis zu $80^{\circ}$ nach oben abgewinkelt (Proband A Monoflosse und Proband Mares Volo). Die Monoflosse war Proband A zu klein. Es ist zu vermuten, dass er in der abgewinkelten Position die für inn angenehmste Haltung fand. Dadurch war die Flosse um die Unterschenkellänge parallel versetzt über ihm. Diese gilt für die ersten beiden Geschwindigkeitsstufen. Danach streckte er die Beine und nahm eine strömungsgünstigere Haltung ein. Dadurch kam es bei der Zusammenstellung der Ergebnisse zu einer polynomischen Funktion mit einer negativen Exponention. Aus diesem Grund wurde diese Datenreihe nicht weiter berücksichtigt. Bei Proband B mit der Mares Volo ist genau das Gleiche zu beobachten. Auch er winkelte die Beine in den Kniegelenken bis zu ca. $80^{\circ}$ nach oben ab. Ursächlich lässt sich eine Bewegungseinschränkung durch den Füßling annehmen, mit welchem Proband $A$ wohl besser zurecht kam. Dadurch werden in dieser Versuchsreihe für Proband B mit der Mares Volo zu hohe passive Kraftwerte gemessen.

\subsubsection{Die Spiroergometrie unter Wasser (Messreihe 4)}

Der Druckminderer und der Atemregler erfüllten über die Gesamtheit der Versuche gesehen die erwarteten Leistungen: Sie lieferten Luft unter Umgebungsdruck und ermöglichten die Ableitung der Ausatemluft unter Umgebungsdruck. Jedoch kam es zu einer teilweise hohen Streuung der Daten auf einzelnen Belastungsstufen. Bis auf einen Versuch waren alle Versuche davon betroffen.

Wie bereits angesprochen, sind auf einigen Belastungsstufen Schwankungen zu erkennen, die nicht mit dem Einschwingverhalten zu erklären sind, da sie im Zeitraum 
erwarteter stabiler Werte liegen. Das liegt an den bereits genannten Gründen (siehe 66ff) und kann auf die Aussage reduziert werden, dass das System Mensch in der untersuchten Belastungssituation mit der verwendeten Technik der Ausatemgasableitung nur bedingt so konstante Werte liefert, wie sie nach einem Einschwingverhalten zu erwarten wären. Gute Beispiele für stabile Werte sind die Versuche Proband A mit Seemann Sub SF 2 und Mares Volo. Bei den Versuchen Proband B mit der Seemann Sub SF 2 ist die erste Belastungsstufe $(-5 \mathrm{~N})$, mit der Mares Volo und der Sporasub Dessault ist die zweite Belastungsstufe $( \pm 0 \mathrm{~N})$ und mit der Monoflosse die letzte Belastungsstufe $(+10 \mathrm{~N})$ nicht stabil. Bei Proband A mit der Sporasub Dessault ist es ebenfalls die letzte Belastungsstufe $(+10 \mathrm{~N})$, in welcher instabile Werte gemessen wurden. Die gleichen Schwierigkeiten wurden von WITTEN $(2000,37)$ mit einem vergleichbaren Versuchsaufbau geschildert und auf eindringendes Wasser zurückgeführt. In der hier vorliegenden Untersuchung kam es nicht zu Wassereinbrüchen, was die Begründung von WITTEN für die Schwankungen nicht bestätigt. Wassertröpfchen im System haben sich aber nicht vermeiden lassen. Dabei ist aber nicht nachzuvollziehen, wie diese über eine $4 \mathrm{~m}$ lange, aufsteigende Schlauchleitung, zur Messeinheit gelangen können. In nur zwei Fällen lagen diese Schwankungen in der letzten Belastungsstufe. Das bedeutet, dass, wenn Wasser im Laufe der anderen betroffenen Versuche eingedrungen ist, das Wasser lediglich punktuell Einfluss auf die Sensorik gehabt hätte, was gleichfalls bedingt nachvollziehbar ist.

Auch eine Schwankung der Ausatemdrücke, ausgelöst durch die Konstruktion der Membranen, kann nicht als Grund genannt werden, da in den Messungen von SCHMIDTS (Anhang 8.1.2) konstante Aus- und Einatemdrücke festgestellt wurden, die einen Einsatz des umgebungsdruckgesteuerten Ausatemventils auch in Tiefen bis zu $30 \mathrm{~m}$ zulassen würden. Andererseits sind Probleme in der Realität aufgetreten und führten zu großer Streuung der Messwerte auf einzelnen Belastungsstufen. Hier scheint eine detaillierte Analyse des umgebungsdruckgesteuerten Ausatemventils in der Praxis angezeigt.

In zwei Versuchen lösten sich die Verbindungen, so dass die Versuche unterbrochen werden mussten. Nach der Unterbrechung ergaben sich in der folgenden Belastungsstufe Werte, die in Verbindung mit den anderen Belastungsstufen weder linear noch polynomisch sinnvoll abzubilden waren und in dieser Belastungsstufe deshalb unberücksichtigt blieben. Als Schlussfolgerung ist in Zukunft darauf zu achten, dass ein Versuch immer ohne Unterbrechung durchzuführen ist.

Die Ausatemmembranen befanden sich direkt am Ohr der Probanden. Die Membranen kamen bei der Ausatmung in Schwingung und erzeugten ein unangenehmes Brummgeräusch, welches durch die hohe Schallübertragung und die Nähe der Membran zum Ohr der Probanden von innen beiden als sehr störend empfunden wurde. Das Problem konnte im Ablauf der Versuche durch modifiziertes, weniger straffes Aufziehen der Membranen vermindert werden.

Die Methode setzt voraus, dass die Probanden im Flossenschwimmen geübt sind. Daher empfiehlt sich eine Anwendung nur bei entsprechender Erfahrung und 
Leistungsfähigkeit der Probanden im Flossenschwimmen, die es ihnen gestattet einen Versuch erfolgreich zu absolvieren. Insbesondere sind folgende Erwartungen zu erfüllen:

- geringes seitliches und vertikales Abdriften,

- kein Schwimmen nach vorne oder Treiben lassen nach hinten,

- Einhalten einer konstanten, strömungsgünstigen Haltung.

Allerdings sind auf einigen Belastungsstufen Schwankungen zu erkennen, die nicht mit dem Einschwingverhalten zu erklären sind, da sie im Zeitraum erwarteter stabiler Werte liegen. Gründe für diese Messschwankungen können verursacht werden durch mehrmaliges starkes Ausatmen des Probanden mit der Folge, dass die Messwerte beeinflusst werden.

Gesondert ist festzuhalten,

- dass der Versuch (Proband B Mares Volo) abgebrochen werden musste, da die kardanische Vorrichtung zur Spiroergometrie im Wasser einen Defekt hatte und ausgetauscht werden musste. Er wurde direkt danach wiederholt.

- dass die Versuche Proband B Mares Volo und Proband A Mares Volo jeweils kurzzeitig unterbrochen werden mussten, da bei beiden Versuchen am Atemregler ein Defekt auftrat. Die daraus folgenden Probleme sind in diesem Kapitel bereits vorgestellt worden.

- dass der erste Versuch Proband B Seemann Sub SF 2 nicht in der vorgesehenen Weise ablief, d.h. die Belastungsstufen waren kürzer als geplant. Da diese Probleme noch vor Ort bemerkt wurden, wurde der Versuch am nächsten Tag wiederholt.

- dass alle anderen Versuche regulär liefen.

Zusammenfassend lässt sich sagen, dass die Versuchsreihe zur Spiroergometrie im Strömungskanal erfolgreich verlief. Die Methode ist geeignet, die $\mathrm{O}_{2}-$ Aufnahme bei Tauchern zu messen und besser geeignet als die von DWYER (1983) vorgestellte Methode, bei der in Zeitabständen Proben asserviert wurden. 


\subsection{Diskussion der Ergebnisse}

\subsubsection{Bestimmung der Strömungsverhältnisse im Kanal (Messreihe 1) und die Kalibrierung des Kraftaufnehmers}

Die Messungen der Strömungsgeschwindigkeit ergaben die in Hypothese 1 vermuteten turbulenten Verhältnisse. Wie erwartet stimmten sie nicht mit den am Kanal vorgegebenen Werten überein. Es erscheint vor Versuchen sinnvoll die Strömungssituation für den Bereich, in dem sich die Probanden aufhalten exakt zu bestimmen. Der Kraftaufnehmer wurde kalibriert und die Formel Umrechnung der Werte eindeutig bestimmt.

\subsubsection{Bestimmung der Strömungswiderstände der Probanden mit unterschiedlichen Auftriebskörpern (Messreihe 2)}

Die Kurven zeigen wie bei Karpovich, Counsilman und Schramm (Abb. 5) die exponentielle Funktion der Kraft der Widerstandserhöhung.

Die Kurven der einzelnen Auftriebskörper sind für beide Probanden annähernd deckungsgleich. Einzige der Messpunkt für die Kragenweste auf der letzten Geschwindigkeitsstufe weist Abweichungen auf. Verglichen mit den Ergebnissen von Karpovich, Counsilman und Schramm, in UNGERECHTS $(1978,67)$, liegen die hier erhobenen Werte deutlich über den Kraftwerten, die diese Autoren für Schwimmer ermittelt haben. Bei $1,0 \mathrm{~m} \cdot \mathrm{s}^{-1}$ geben sie Werte von 25 bis $35 \mathrm{~N}$ an. Für die gleiche Geschwindigkeit errechnen sich auf Basis dieser Untersuchung Werte zwischen 87,70 $\mathrm{N}$ für Proband $\mathrm{B}$ mit dem Jacket und 106,58 $\mathrm{N}$ für Proband $\mathrm{A}$ mit der Kragenweste. Vergleichbare Werte für Ausrüstungskonfigurationen, die denen von Tauchern entsprechen, liegen in der Literatur nicht vor.

Das Jacket, das Wing Jacket, das HUB und die Kragenweste sind bezogen auf den Strömungswiderstand bei ansteigender Geschwindigkeit Ausrüstungsgegenstände mit unterschiedlichen Kraftwerten $F_{w}$. Die Messungen ergeben fast identische Kraftverläufe für das Wing Jacket sowie ähnliche Widerstandsverläufe bei beiden Probanden. Auffallend ist, dass alle Kraftwerte bis $0,8289 \mathrm{~m} \cdot \mathrm{s}^{-1}$ annähernd identisch sind. Deutliche Unterschiede treten erst bei $1,1283 \mathrm{~m} \cdot \mathrm{s}^{-1}$ und $1,3951 \mathrm{~m} \cdot \mathrm{s}^{-1}$ auf. Das lässt den Schluss zu, dass bei normaler taucherischer Beanspruchung, welche mit 0,4 - 0,5 $\mathrm{m} \cdot \mathrm{s}^{-1}$ in der Literatur angegeben wird, für den Taucher keine nennenswerten Unterschiede bezüglich des Widerstandes bei den gemessenen Auftriebskörpern bestehen HOFFMANN $(2002,192)$.

Das legt den Schluss nah, dass unterschiedliche Auftriebskörper zu unterschiedlichen Belastungen für den Taucher beim Schwimmen gegen Strömung oder ähnlichen Grenzsituationen führen. Weiter kann angenommen werden, dass zusätzliche Ausrüstungsgegenstände wie Trockentauchanzüge, Lampen, Reels, Stage-Flaschen, 
Kameraausrüstung, Hebesäcke oder auch ein gefundener Bleigurt zu einer Widerstanderhöhung führen können, deren Untersuchung von Interesse ist.

Hergeleitet aus

$$
F_{D p}=c \cdot \frac{\rho}{2} \cdot v^{2} \cdot A
$$

liegt die Vermutung nahe, dass unterschiedliche Auftriebskörper im Wesentlichen zu einer Veränderung des Wertes A, also der Stirnfläche, führen, da alle anderen Größen durch den veränderten Ausrüstungsgegenstand nicht beeinflusst werden und durch den Versuchsaufbau als konstant zu betrachten sind.

- Beim HUB ist zu vermuten, dass durch die Abdeckung der Schläuche und Zusatzausrüstung eine derartige Volumenvergrößerung auftritt, dass sich damit die Stirnfläche und $F_{W}$ ungünstig verändert.

- Die Kragenweste weist ähnlich hohe Werte auf. Das mag darin begründet sein, dass die herabhängende Westenflasche zur Vergrößerung der Stirnfläche beiträgt.

- Als Grund für die niedrigen Werte des Wing Jackets kann angeführt werden, dass, obwohl es ein optisch großes Volumen hat, dieses im Wasser nicht wirksam ist. Es scheint so, dass die Volumina ihren Querschnitt in Bereichen haben, die hinter, also im Strömungsschatten von anderen Stirnflächen liegen. Dies mag vor allem hinter den Schultern sein.

- Das einfache Jacket legt sich eng dem Körper an und führt so zur geringsten Vergrößerung der Stirnfläche.

Diese Ergebnisse lassen die Annahme $z u$, dass in den normalen Geschwindigkeitsbereichen für Sporttaucher bis zu gemessenen 0,8289 $\mathrm{m} \cdot \mathrm{s}^{-1}$ keine gravierenden unterschiedliche Strömungswiderstände auf Grund der Auftriebskörpers entstehen. Die maximalen Differenzen betragen 12,9 N zwischen dem Versuch Proband B Jacket mit 61,50 N und dem Versuch A Kragenweste mit 74,40 N. Daraus lassen sich keine voneinander abweichenden risikorelevanten $\mathrm{zu}$ erbringenden Leistungsunterschiede bezüglich der Auftriebskörper ableiten. Eine eindeutige Reihenfolge der Maxima ist in diesen drei Stufen nicht möglich, was darauf schließen lässt, dass die Körperhaltung in diesen Bereichen einen relevanten Einfluss hat. In den Geschwindigkeitsstufen mit $1,1283 \mathrm{~m} \cdot \mathrm{s}^{-1}$ und $1,3951 \mathrm{~m} \cdot \mathrm{s}^{-1}$ bestehen mit bis zu 55,69 Watt Differenz zwischen den Ausrüstungsgegenständen erhebliche sicherheitsrelevante Unterschiede in den daraus abzuleitenden Leistungsanforderungen an die Probanden, die dem Ausrüstungsgegenstand Auftriebskörper zuzuordnen sind.

Aus den Untersuchungen können für den Sporttaucher Empfehlungen für die Wahl eines Ausrüstungsgegenstandes unter der Prämisse der bei Strömung zu erbringenden Leistung gegeben werden. Handlungsanweisungen, die zur Sicherheit des Tauchers bei starker Strömungsexposition beitragen, lassen sich ebenso ableiten: 
Auf Grund der gesteigerten Leistungsanforderungen bei starker Strömung ist es besser, sofern die Möglichkeit besteht, sich festzuhalten, als gegen die Strömung anzukämpfen. Notfalls ist es vorzuziehen, sich abtreiben zu lassen, als gegen eine Strömung anzukämpfen.

Diese Handlungsanweisungen sind durch die Ergebnisse dieser Untersuchung belegt.

Beim Tauchen findet mit zunehmender Tiefe eine Verlagerung der Volumina vom Kälteschutzanzug in den als Tariermittel mitgeführten Auftriebskörper statt. Ob diese Verlagerung auch zu einer Veränderung der Stirnfläche A und damit zur Widerstandserhöhung führt, wäre zu untersuchen.

\subsubsection{Bestimmung der Kraftwerte für unterschiedliche Flossen (Messreihe 3)}

Grundsätzlich bestätigt die Untersuchung die Ergebnisse von KARPOVICH (1939) und HOLMÉR (1974), die $F_{w}=29 \cdot v^{2}$ fanden. In diesen Untersuchungen wurden Schwimmer an der Wasseroberfläche untersucht. Die hier vorliegenden Ergebnisse beziehen sich auf Schwimmtaucher mit Flossen. Einflüsse der Flossen sind festzustellen.

Es treten in den ersten beiden Geschwindigkeitsstufen die gleichen, die Wasserlage beeinflussenden, Phänomene auf, die bereits bei den Untersuchungen zu verschiedenen Auftriebskörpern zu beobachten waren. Dadurch sind in der ersten Geschwindigkeitsstufe zwischen $145 \%$ und $465 \%$ erhöhte Kraftwerte festzustellen. Mit verbesserter Gleitlage durch die ansteigende Geschwindigkeit in den folgenden Stufen verringert sich der prozentuale Anstieg bis hin zu 4\% bis $50 \%$ in der letzten Geschwindigkeitsstufe. Insofern sind die Kraftwerte im Vergleich mit den beiden genannten Untersuchungen in den ersten beiden Geschwindigkeitsstufen, bedingt durch die schlechte Gleitlage, als deutlich erhöht und bis zu den letzten Stufen als erhöht zu bezeichnen. In keinem Fall wird mit Flossen ein niedriger Wert ermittelt als er in den Untersuchungen von KARPOVICH et al. (1939) und HOLMÉR (1974) genannt ist. In allen Geschwindigkeitsstufen führt also der zusätzliche Ausrüstungsgegenstand Flossen zu einer Widerstandserhöhung. Mit der gleichen Argumentation werden die Messergebnisse von Karpovich, Counsilman und Schramm, in UNGERECHTS (1978, $67)$ mit Werten von 25 bis $35 \mathrm{~N}$ bei $1,0 \mathrm{~m} \cdot \mathrm{s}^{-1}$ bestätigt. 


\begin{tabular}{|l|c|c|c|c|c|}
\hline Proband A & $0,3567 \mathrm{~m} \cdot \mathrm{s}^{-1}$ & $0,5817 \mathrm{~m} \cdot \mathrm{s}^{-1}$ & $0,8289 \mathrm{~m} \cdot \mathrm{s}^{-1}$ & $1,1283 \mathrm{~m} \cdot \mathrm{s}^{-1}$ & $1,3951 \mathrm{~m} \cdot \mathrm{s}^{-1}$ \\
\hline Mares Volo & $\mathrm{F}_{\mathrm{Dp}} /(\mathrm{N})$ & $\mathrm{F}_{\mathrm{Dp}} /(\mathrm{N})$ & $\mathrm{F}_{\mathrm{Dp}} /(\mathrm{N})$ & $\mathrm{F}_{\mathrm{Dp}} /(\mathrm{N})$ & $\mathrm{F}_{\mathrm{Dp}} /(\mathrm{N})$ \\
\hline Seemann Sub SF 2 & 10,77 & 20,08 & 33,63 & 55,34 & 83,53 \\
\hline Sporasub Dessault & 20,88 & 23,49 & 31,30 & 49,42 & 66,25 \\
\hline Monoflosse & 14,55 & 18,10 & 30,56 & 47,96 & 73,73 \\
\hline $\begin{array}{l}\text { KARPOVICH et al. (1939) } \\
\text { HOLMÉR (1974) }\end{array}$ & 28,91 & 31,18 & 42,08 & 48,93 & 64,20 \\
\hline \begin{tabular}{l}
$\mathrm{F}_{\mathrm{Dp}}=29 \cdot \mathrm{v}^{2}$ \\
\hline
\end{tabular} & 3,69 & 9,81 & 19,93 & 36,92 & 56,45 \\
\hline
\end{tabular}

Tabelle 18: $\quad F_{D p}$ mit Flossen für Proband $A$ im Vergleich mit den Ergebnissen von KARPOVICH et al. (1939) und HOLMÉR (1974).

\begin{tabular}{|l|c|c|c|c|c|}
\hline Proband B & $0,3567 \mathrm{~m} \cdot \mathrm{s}^{-1}$ & $0,5817 \mathrm{~m} \cdot \mathrm{s}^{-1}$ & $0,8289 \mathrm{~m} \cdot \mathrm{s}^{-1}$ & $1,1283 \mathrm{~m} \cdot \mathrm{s}^{-1}$ & $1,3951 \mathrm{~m} \cdot \mathrm{s}^{-1}$ \\
\hline & $\mathrm{F}_{\mathrm{Dp}} /(\mathrm{N})$ & $\mathrm{F}_{\mathrm{Dp}} /(\mathrm{N})$ & $\mathrm{F}_{\mathrm{Dp}} /(\mathrm{N})$ & $\mathrm{F}_{\mathrm{Dp}} /(\mathrm{N})$ & $\mathrm{F}_{\mathrm{Dp}} /(\mathrm{N})$ \\
\hline Mares Volo & 10,96 & 20,61 & 45,17 & 64,57 & 84,53 \\
\hline Seemann Sub SF 2 & 11,34 & 14,64 & 25,17 & 44,85 & 58,96 \\
\hline Sporasub Dessault & 9,05 & 15,38 & 25,91 & 39,49 & 64,35 \\
\hline Monoflosse & 18,06 & 21,34 & 33,75 & 45,97 & 68,07 \\
\hline $\begin{array}{l}\text { KARPOVICH et al. (1939) } \\
\text { HOLMÉR (1974) }\end{array}$ & 3,69 & 9,81 & 19,93 & 36,92 & 56,45 \\
\hline $\begin{array}{l}\text { Fo } \\
\text { Fo } 29 \cdot \mathrm{v}^{2}\end{array}$ & & & & \\
\hline
\end{tabular}

Tabelle 19: $\quad F_{D p}$ mit Flossen für Proband $B$ im Vergleich mit den Ergebnissen von KARPOVICH (1939) und HOLMÉR (1974). 


\subsubsection{Die Spiroergometrie unter Wasser (Messreihe 4)}

In dieser Untersuchung wurden Werte für $\dot{V} \mathrm{O}_{2}$ von $906 \mathrm{ml} \cdot \mathrm{min}^{-1}$ bis $2,994 \mathrm{ml} \cdot \mathrm{min}^{-1}$ gemessen. Der Vergleich mit vorliegenden Untersuchungen stellt sich wie folgt dar:

\begin{tabular}{|c|c|c|c|}
\hline Autor & Jahr & $\begin{array}{c}\dot{V} \mathrm{O}_{2} / \\
\left(\mathrm{ml} \cdot \mathrm{min}^{-1}\right) \\
\end{array}$ & Besonderheiten \\
\hline \multirow[t]{2}{*}{ DONALD, DAVIDSON } & \multirow[t]{2}{*}{1954} & 3600 & \multirow{2}{*}{$\begin{array}{l}\text { Bei Schwimmtauchern } \\
\text { Bei Helmtauchern }\end{array}$} \\
\hline & & 2350 & \\
\hline KRASTEV & 1964 & 1660 & Douglas-Haldane-Verfahren \\
\hline FOLEY & 1967 & $907-2940$ & $\begin{array}{l}\text { Asservation im Vakuumtank. } \\
\text { Veränderung in Abhängigkeit zur } \\
\text { Schwimmgeschwindigkeit }\end{array}$ \\
\hline \multirow[t]{2}{*}{ PILMANIS } & \multirow[t]{2}{*}{1977} & 3280 & \multirow{2}{*}{$\begin{array}{l}\text { In } 10 \quad \mathrm{~m} \text { Wassertiefe tauchend } \\
\text { gegen } 24 \mathrm{~N} \\
\text { In } 30 \mathrm{~m} \text { Wassertiefe tauchend } \\
\text { gegen } 24 \mathrm{~N}\end{array}$} \\
\hline & & 3470 & \\
\hline DRÄGER & 1999 & $2090-2590$ & Geschwindigkeitsabhängig \\
\hline PENDERGAST et al. & 2003 & $951-2949$ & Geschwindigkeitsabhängig \\
\hline $\begin{array}{l}\text { Zusatzkraftbeaufschlag- } \\
\text { ung bei } v=\text { konstant }\end{array}$ & 2004 & $906-2990$ & In Abhängigkeit zur Zusatzkraft \\
\hline
\end{tabular}

Tabelle 20: $\quad \dot{V} \mathrm{O}_{2}$ in verschiedenen Untersuchungen.

In dieser Untersuchung wurde mit konstanter Geschwindigkeit bei variabler Zusatzkraft geschwommen. Die Ergebnisse bestätigen die Daten vorliegender Untersuchungen. Methodisch vergleichbar sind die Untersuchung von FOLEY (1967) und PENDERGAST et al. (2003), da hier mit veränderten Geschwindigkeiten geschwommen wurde. Alle anderen Untersuchungen gehen entweder von einem konstanten Widerstand aus, der zu überwinden ist PILMANIS (1977), von einer konstanten Geschwindigkeit, welche die Probanden wählen können DRÄGER (1999) oder von nicht standardisierten Tätigkeiten DONALD, DAVIDSON (1954) und KRASTEV (1964).

Gleichzeitig wurden Ruheumsätze von 360 bis $520 \mathrm{ml} \cdot \mathrm{min}^{-1}$ gefunden.

\subsubsection{Respiratorischer Quotient}

Keiner der Probanden war in den Versuchen ausbelastet, da der RQ maximal bis 0,94 anstieg. Dieser Wert wurde in zwei Versuchen erreicht, was deutlich unter der 
Ausbelastungsgrenze von $R Q=1$ liegt. Dieser müsste erwartungsgemäß bei ansteigender Belastung gegen 1 ansteigen BRAUER/GOTTSCHLALK $(1996,138)$.

Nur in vier Fällen ist der eigentlich zu erwartende, gleichförmige bis lineare Anstieg zu erkennen (B/Mares Volo, A/Mares Volo, B/Seemann Sub SF 2und A/Sporasub Dessault). Die anderen drei RQ-Kurven sind in ihrer Entwicklung uneinheitlich.

Mehrere Vermutungen sind aus dieser Feststellung abzuleiten:

- Der menschliche Körper ist als Gesamtsystem zu betrachten. Neben der in der spiroergometrischen Untersuchung feststellbaren Reaktion kann der Bewegungsablauf an die Belastung angepasst werden, ebenso, wie die Formgebung und Flexibilität einer Flosse in einem spezifischen Belastungssegment besser optimiert werden kann als in einem anderen, welches unterhalb oder oberhalb des optimalen Belastungssegmentes liegen kann.

- Höhere Körpermasse scheint bei kleinen Flossen dazu zu führen, dass fehlender Flossenschub nur über erhöhte Kreislauftätigkeit kompensiert werden kann (Proband A mit Seemann Sub SF 2).

Eine bei STEGEMANN $(1991,60)$ angesprochene mögliche Hyperventilation und damit eine Verschiebung des $R Q$ zu höheren Werten hat nicht stattgefunden. Diese Hyperventilation kann bei einem ungeübten Probanden auftreten. Da beide Probanden erfahrene Flossenschwimmer und dadurch an das Atmen durch Mundstücke (Atemregler oder Schnorchel) gewöhnt sind, wäre eine derartig begründete Verschiebung des $R Q$ nicht erklärbar.

\subsubsection{Wirkungsgrad}

GOFF (1957) fand Wirkungsgrade zwischen $1,2 \%$ und $5,6 \%$ für das Tauchen. COSTILL (1971) gab in seiner Untersuchung einen Bruttowirkungsgrad von $4,0 \%$ bis $5,7 \%$ an. In dieser Untersuchung wurden Nettowirkungsgrade zwischen 3,65\% und $8,64 \%$ und Bruttowirkungsgrade zwischen $2,61 \%$ und $6,85 \%$ gefunden. Die Wirkungsgrade stehen in Abhängigkeit zu der jeweils geschwommenen Flosse.

So sind die Wirkungsgrade für die Mares Volo bei beiden Probanden fast identisch. Bei der Sporasub Dessault liegen sie netto zwischen $0,2 \%$ und 1,2\% und brutto zwischen $0,5 \%$ und $0,05 \%$ auseinander. Hier haben beide Probanden sehr ähnliche Wirkungsgrade aufzuweisen. Bei der Seemann Sub SF 2 sind die Unterschiede deutlich, netto um $1,2 \%$ und brutto von $0,6 \%$ bis $0,8 \%$. Hier scheint das Verhältnis von Körpermasse zu Flossenoberfläche, welches bei Proband $A$ ungünstiger ist (Proband $A$ $78 \mathrm{~kg}$, Proband B $68 \mathrm{~kg}$ ), in Kombination mit einer im Verhältnis zu den anderen Flossen weichen Flosse, verantwortlich zu sein. 


\subsubsection{Mechanische Leistung}

NIKLAS (2000) verglich die Leistung von Tauchern bei verschiedenen Über- und Unterwasserergometrien bezüglich der Sauerstoffaufnahme. In Tabelle 21 sind die von NIKLAS erhobenen Daten für das Tauchen den hier gefundenen Ergebnissen gegenübergestellt.

\begin{tabular}{|c|c|c|c|c|c|c|c|c|}
\hline & Niklas & \multicolumn{7}{|c|}{ Mechanische Nettoleistung mit unterschiedlichen Flossen } \\
\hline $\begin{array}{c}\dot{V} \mathrm{O}_{2} \\
/ \\
\left(\mathrm{ml} \cdot \mathrm{min}^{-1}\right)\end{array}$ & $\begin{array}{c}\text { UW- } \\
\text { Schwimm- } \\
\text { ergometrie } \\
\quad / \\
\left(\mathrm{P}_{\text {mech }} \cdot \mathrm{W}^{-1}\right)\end{array}$ & $\begin{array}{c}B \\
\text { Monoflosse } \\
/ \\
\left(\mathrm{P}_{\text {mech }} \cdot \mathrm{W}^{-1}\right)\end{array}$ & $\begin{array}{c}\text { B } \\
\text { Sporasub } \\
\text { Dessault / } \\
\left(\mathrm{P}_{\text {mech }} \cdot \mathrm{W}^{-1}\right)\end{array}$ & $\begin{array}{c}A \\
\text { Sporasub } \\
\text { Dessault / } \\
\left(\mathrm{P}_{\text {mech }} \cdot \mathrm{W}^{-1}\right)\end{array}$ & $\begin{array}{c}B \\
\text { Seemann } \\
\text { Sub SF 2 I } \\
\left(P_{\text {mech }} \cdot W^{-1}\right)\end{array}$ & $\begin{array}{c}A \\
\text { Seemann } \\
\text { Sub SF 2 / } \\
\left(P_{\text {mech }} \cdot W^{-1}\right)\end{array}$ & $\begin{array}{c}\mathrm{B} \\
\text { Mares Volo } \\
/ \\
\left(\mathrm{P}_{\text {mech }} \cdot \mathrm{W}^{-1}\right)\end{array}$ & $\begin{array}{c}\mathrm{A} \\
\text { Mares Volo } \\
/ \\
\left(\mathrm{P}_{\text {mech }} \cdot \mathrm{W}^{-1}\right)\end{array}$ \\
\hline 180 & & & & & & & & \\
\hline 250 & & & & & & & & \\
\hline 500 & 0 & & & & & & & \\
\hline 650 & & & & & & & & \\
\hline 1000 & 13 & & 12 & & & & & \\
\hline 1500 & 18 & & 22 & 20 & 20 & & 14 & \\
\hline 2000 & 24 & 45 & & & & 22 & 20 & \\
\hline 2500 & 33 & & & & & 29 & & 25 \\
\hline
\end{tabular}

Tabelle 21: $\quad$ Vergleich der Ergebnisse dieser Untersuchung mit den Ergebnissen von NIKLAS (2000) zur UW-Schwimmergometrie. Mit freundlicher Genehmigung des Autors.

Bis auf die Monoflosse sind die mechanischen Leistungen annähernd identisch.

Deutlich wird, dass bereits geringe mechanische Leistungen unter Wasser einer hohen Sauerstoffaufnahme bedürfen. Bei einem $\dot{V} \mathrm{O}_{2}$ von $2000 \mathrm{ml} \cdot \mathrm{min}^{-1}$, welcher dem $\dot{V} \mathrm{O}_{2 \max }$ von untrainierten jungen Menschen entspricht STEGEMANN $(1991,263)$, werden lediglich 20 - 45 Watt mechanisch unter Wasser geleistet. In der arbeitsmedizinischen Beurteilung von Berufstauchern und Freizeittauchern wird die Fahrradergometrie eingesetzt WELSLAU (1977), WENDLING et al. (2001), welche mit 20 bis $25 \%$ einen deutlich höheren Wirkungsgrad hat und zudem die Belastungssituation unter Wasser nicht adäquat abbildet STEINBACH et al. (1985), HOLMÉR, ÅSTRAND (1972), BRÄUER (1992), BRÄUER et al. (1994).

Auf Grund dieser Konstellation sind Fehlbeurteilungen in der arbeitsmedizinischen Beurteilung von Tauchern wegen eben dieser nicht adäquaten Untersuchungsmethode zu erwarten. Zur Absicherung der Taucher wäre die hier vorgestellte Methode geeignet. Sie führt zu einer auf den Einsatzbereich unter Wasser bezogenen Untersuchungsaussage. 


\subsubsection{Dynamischer Widerstand}

Die in Messreihe 3 ermittelten Mittelwerte F / (N) bei der Geschwindigkeitsstufe 0,7288 $\mathrm{m} \cdot \mathrm{s}^{-1}$ entsprechend betragen:

\begin{tabular}{|l|c|c|c|c|}
\hline & Proband A & Proband A & Proband B & Proband B \\
\hline & $F_{\mathrm{Dp}} /(\mathrm{N})$ & $\mathrm{F}_{\mathrm{Dd}} /(\mathrm{N})$ & $\mathrm{F}_{\mathrm{Dp}} /(\mathrm{N})$ & $\mathrm{F}_{\mathrm{Dd}} /(\mathrm{N})$ \\
\hline Seemann Sub SF2 & 30,46 & 30,81 & 22,33 & 24,76 \\
\hline Sporasub Dessault & 25,95 & 30,74 & 20,84 & 20,62 \\
\hline Mares Volo & 27,68 & 33,97 & 33,80 & 21,38 \\
\hline Monoflosse & & & 29,04 & \\
\hline
\end{tabular}

Tabelle 22: $\quad$ Mittelwerte $F_{D p}$ und $F_{D d}$ bei $0,7288 \mathrm{~m} \cdot \mathrm{s}^{-1}$ für beide Probanden.

Die Mittelwerte $F_{D p}[N]$ bei der Geschwindigkeitsstufe $0,9426 \mathrm{~m} \cdot \mathrm{s}^{-1}$ für Proband $B$ und die Monoflosse sind in der folgenden Tabelle 23 dargestellt.

\begin{tabular}{|l|c|c|}
\hline & Proband B & Proband B \\
\hline & $\mathrm{F}_{\mathrm{Dp}}[\mathrm{N}]$ & $\mathrm{F}_{\mathrm{Dd}}[\mathrm{N}]$ \\
\hline Monoflosse & 39,56 & 50,12 \\
\hline
\end{tabular}

Tabelle 23: $\quad$ Mittelwerte $F_{D p}$ und $F_{D d}$ bei $0,9426 \mathrm{~m} \cdot \mathrm{s}^{-1}$ für Proband $B$.

Die Werte für die dynamische Widerstandskraft sind über die Extrapolation der Arbeitssauerstoffaufnahme auf die Abszisse des Widerstandes ermittelt worden. Erwartet wurde, dass $F_{D d}$ größer ist als $F_{D p}$. In vier von sieben Versuchen wurde diese Annahme bestätigt. In zwei Versuchen waren $F_{D d ~}$ und $F_{D p}$ identisch oder war $F_{D d}$ geringfügig niedriger als $F_{\mathrm{Dp}}$. In einem Versuche wurde die Annahme nicht bestätigt. Hier war $F_{D d}$ deutlich niedriger als $F_{D p}$.

Es ist zu berücksichtigen, dass in den Versuchen zur Ermittlung der passiven Widerstandskräfte die Flossen in den ersten beiden Geschwindigkeitsstufen herabhingen, dadurch die Stirnfläche erhöht wurde und dadurch in nicht feststellbarem Maße in diesen Geschwindigkeitsstufen erhöhte Werte gemessen wurden. Hier muss der Versuchsaufbau so modifiziert werden, dass der Ansatzpunkt der Schleppkraft nicht vor dem Körper, sondern in der Körpermitte ist. Dadurch wird möglicherweise vermieden, dass die Flossen herabhängen und sich die Stirnfläche vergrößert. Schlussfolgernd ist $\mathrm{zu}$ vermuten, dass die gemessenen Werte für $F_{D p}$ aus den beschriebenen Gründen zu hoch sind.

Unter diesen Voraussetzungen betrachtet, erscheint das Verfahren mittels der Zusatzkraftbeaufschlagung und der Regression der Geraden der Sauerstoffaufnahme 
auf die Achse der Kraftwerte geeignet, die dynamische Widerstandkraft eines Tauchers abzubilden.

Dieses wird auch durch den Vergleich mit vorliegenden Untersuchungen zum dynamischen Widerstand von Schwimmern und Flossenschwimmern an der Wasseroberfläche deutlich. Im Vergleich mit der Untersuchung von DI PRAMPERO et al. (1974) ist festzustellen, dass der Unterschied zwischen $F_{D P}$ und $F_{D d}$ beim Flossenschwimmen unter Wasser mit $10,7 \%$ bei $0,7288 \mathrm{~m} \cdot \mathrm{s}^{-1}$ und $26,7 \%$ bei 0,9426 $\mathrm{m} \cdot \mathrm{s}^{-1}$ unter dem von DI PRAMPERO et al. angegebenen Unterschied von 30\% liegt. Hier bestätigt sich die Annahme, dass die Vorhalte der Arme und die ruhigere Wasserlage des Schwimmtauchers unter Wasser gegenüber der Wasserlage des Kraulschwimmer an der Wasseroberfläche zu einem geringeren $F_{D d}$ führt.

Der hohe Wert für die Geschwindigkeit $0,9426 \mathrm{~m} \cdot \mathrm{s}^{-1}$ erklärt sich mit der in diesem Versuch geschwommenen Monoflosse.

\begin{tabular}{|l|l|c|c|c|c|}
\hline Untersuchung & Besonderheiten & & & & \\
\hline & & $\begin{array}{c}\mathrm{v} \\
/\left(\mathrm{m} \cdot \mathrm{s}^{-1}\right)\end{array}$ & $\begin{array}{c}\mathrm{F}_{\mathrm{DP}} \\
/(\mathrm{N})\end{array}$ & $\begin{array}{c}\mathrm{F}_{\mathrm{Dd}} \\
/(\mathrm{N})\end{array}$ & Differenz \\
\hline DI PRAMPERO & Kraulschwimmer & 0,55 & $12-22$ & $23-45$ & $30 \%$ \\
et al. (1974) & an der & 0,90 & $27-44$ & $44-81$ & \\
& Wasseroberfläche & & & $28,3 \pm 10,8$ & \\
ZAMPARO & Flossenschwimmer & 0,7 & & $22,9 \pm 5,4$ & \\
$($ 2002) & an der & 0,8 & & $41,8 \pm 13,5$ & \\
& Wasseroberfläche & 0,9 & & $41,9 \pm 10,3$ & \\
& & 1,0 & & $54,1 \pm 6,6$ & \\
& & 1,1 & & & \\
\hline & & & & $28,18^{27}$ & $10,7 \%$ \\
& & 0,7288 & 25,45 & $26,7 \%$ \\
\hline
\end{tabular}

Tabelle 24: $\quad$ Vergleich der Ergebnisse der Untersuchungen von DI PRAMPERO et al. (1974) und ZAMPARO et al. (2002) mit den Ergebnissen dieser Untersuchung zum dynamischen Widerstand $\mathrm{F}_{\mathrm{Dd}}$.

\subsubsection{MET}

Die relativ zum Körpergewicht günstigste Flosse ist bei beiden Probanden die Sporasub Dessault, gefolgt von der Seemann Sub SF 2 und der Mares Volo. Die Reihung ist bei beiden Probanden eindeutig und identisch. Wie zu erwarten ist die Monoflosse bei Proband B die ungünstigste Flosse, wobei der Wert auf Grund der höheren Anstromgeschwindigkeit nicht vergleichbar ist. Dieser kann nicht mit dem Quotienten 0,7288/0,9426 bereinigt werden, da durch den ansteigenden Widerstand keine Linearität gegeben ist.

\footnotetext{
${ }^{27}$ Die Werte von Proband B mit der Mares Volo bleiben auf Grund des bereits beschriebenen Haltungsfehlers unberücksichtigt.
} 


\section{3 Überprüfung der Hypothesen}

1. Im Schwimmkanal der Elbeschwimmhalle in Magdeburg findet sich eine turbulente Strömungssituation. Die erhobenen Daten weichen von den voreingestellten Geschwindigkeiten am Kanal zum Teil erheblich ab.

2. Die passive Widerstandskraft $F_{D p}$ von zwei schwimmtauchenden Probanden, die unterschiedliche Auftriebskörper tragen, ändert sich zum Teil erheblich in Abhängigkeit zum getragenen Ausrüstungsgegenstand. Für die sich daraus ergebende Leistung werden in den ersten drei Geschwindigkeitsstufen geringe (bis maximal 6,77 W) und in den letzten beiden Geschwindigkeitsstufen erhebliche Differenzen (bis maximal $55,69 \mathrm{~W}$ ) festgestellt. Der passive Widerstand von Schwimmtauchern mit unterschiedlichen Flossen wird in Versuchen ermittelt. Der Einfluss von Flossen, die diese Probanden tragen, ist bezogen auf $F_{D p}$ gering. Die Daten sind mit Ergebnissen anderer Untersuchungen vergleichbar.

3. Die Spiroergometrie unter Wasser wird erfolgreich durchgeführt. Die Belastungsparameter und Stoffwechseldaten werden erhoben. In den Versuchen zeigt sich, dass die Probanden mit verschiedenen Flossen unterschiedliche Leistungen zur Egalisierung eines festgelegten Widerstandes erbringen müssen. Auf Grundlage des Brutto- und Nettowirkungsgrades ist die Mares Volo für beide Probanden die ungünstigste Flosse. Mit der Monoflosse werden die höchsten Wirkungsgrade erreicht. Betrachtet auf Basis des MET benötigen beide Probanden für die Sporasub Dessault ein niedrigeres MET als für die Seemann Sub SF 2. Ein noch höheres MET realisieren sie mit der Mares Volo. Die Monoflosse hat das höchste MET.

4. Die Methode der Zusatzkraftbeaufschlagung mit der Regression der Arbeitssauerstoffaufnahme auf die Abszisse mit $\dot{V} \mathrm{O}_{2}=0$ erscheint geeignet, den Wert des dynamischen Widerstandes zu ermitteln. Die Werte finden sich oberhalb des passiven Widerstandes und erscheinen plausibel und hinreichend genau. Die Versuchsaufbauten und Methoden sind objektivierbar, validierbar, standardisierbar und reliabel.

5. Die Methode bildet die Belastungssituation für Schwimmtaucher ab und ist deshalb zur Leistungsdiagnostik von Schwimmtauchern geeignet und kann im Rahmen der Tauchtauglichkeitsuntersuchung eingesetzt werden. Jedoch ist zu bedenken, dass die Methode nur in Strömungskanälen eingesetzt werden kann. Somit steht der erreichten Abbildung der realen Belastungssituation eine ungenügende Realisierbarkeit in der Praxis entgegen. 


\subsection{Zusammenfassung}

In einem Strömungskanal wird die Strömungssituation bestimmt. Es werden Daten für die Strömung in dem Bereich bestimmt, in dem sich die Probanden befinden. Der Kraftaufnehmer wird kalibriert und die Formel zur Umrechnung der Werte eindeutig bestimmt.

Der passive Widerstand von zwei schwimmtauchenden Probanden, die unterschiedliche Auftriebskörper tragen, wird in Versuchen ermittelt. Es werden Unterschiede festgestellt, die einzelnen Auftriebskörpern eindeutig zuzuordnen sind. Die sich daraus ergebenden Leistungen, die Schwimmtaucher mit unterschiedlichen Auftriebskörpern erbringen müssen, werden in Handlungsanweisungen für Tauchausbilder zusammengefasst.

Der passive Widerstand von zwei schwimmtauchenden Probanden, die Flossen tragen, wird in Versuchen ermittelt. Die Kraftwerte differieren in geringem Maße. Ein Einfluss der Körperhaltung insbesondere bei niedrigen Strömungsgeschwindigkeiten wird festgestellt.

Es wird ein Ventil konstruiert, mit dem es möglich ist, die Ausatemluft tauchender Probanden zur weiteren Analyse abzuleiten. Das Ventil stellt in den Versuchen seine Tauglichkeit unter Beweis.

Die Probanden absolvieren schwimmtauchend einen standardisierten Belastungsversuch. $\dot{V} \mathrm{O}_{2}$ und $\dot{V} \mathrm{CO}_{2}$ werden ermittelt. $\dot{V} \mathrm{O}_{2}$ und $\dot{V} \mathrm{CO}_{2}$ stehen in linearer Beziehung zur Zusatzkraft.

Der Bruttowirkungsgrad beträgt zwischen 2,61 \% und 6,85 \%. Der Nettowirkungsgrad beträgt zwischen 3,64 \% und 8,61 \%. MET wird zwischen 8,36 und 31,40 festgestellt. Die dynamische Widerstandskraft $F_{D d}$ ist bei $0,7228 \mathrm{~m} \cdot \mathrm{s}^{-1}$ um $10,7 \%$ größer als die passive Widerstandskraft $F_{D p}$. 
ADRIAN, M.J.; SINGH, M.; KARPOVICH, P.V. (1966): Energy cost of leg kick, arm stroke, and whole crawl stroke. In: J Appl Physiol 21(6), 1763-1766.

ALMELING, M.; BÖHM, F.; WELSLAU, W. (2002a): Handbuch Tauch- und Hyperbarmedizin. Landsberg/Lech.

ALMELING, M. (2002b): Einleitung und generelle Anmerkungen zur Tauchtauglichkeit. In: ALMELING, M.; BÖHM, F.; WELSLAU, W. (2002): Handbuch Tauch- und Hyperbarmedizin. Landsberg/Lech. II-7.1. 1-9.

ALMELING; M. (2004): Med-Habil. Göttingen (in Vorbereitung). Mit freundlicher Genehmigung des Autors.

ANTRIEB IM WASSER (2000). In MIT-Press (Hrsg): Divemaster, Stuttgart 3, 29-34.

ANTRIEB IM WASSER: FLOSSENMODELLE (2000). In MIT-Press (Hrsg): Divemaster, Stuttgart 4, 43- 47.

ANTRIEBSPADDEL (2002). In: Jahr-Verlag(Hrsg): tauchen, Hamburg 10, 110-118.

ÅSTRAND, P.O.; ENGLESSON, S. (1972): A swimming flume. In: J. Appl. Physiol., $\underline{33}$, 4,514

AUSTE, N. (1978): Schwimmen und Tauchen in der Schule. In: Schriftenreihe zur Praxis der Leibeserziehung und des Sports, Band 137, Schorndorf.

BARTMANN, H. (1989):Tauchratgeber Recht. Landsberg.

BARTMANN, H. (2002): Taucherhandbuch. Landsberg.

BASCHTA, M. (2002): Gerätetauchen in der Rehabilitation von Querschnittsgelähmten. In CAISSON $\underline{17}(1-3), 15-19$.

BINNER, U. (1977): Bemerkungen zum Training für das Flossenschwimmen. In: Poseidon 10/1977 S.467

BAUER, A. (1991): Belastung und Beanspruchung beim Wildwasserfahren - eine empirische Untersuchung zur Analyse leistungsphysiologischer Parameter. Dissertation. Göttingen.

BRÄUER, T. (1992): Leistungsdiagnostische Laktat- und Herzfrequenzmessungen bei Tauchern. Kiel.

BRÄUER, T. SIMON, G.; RIECKERT, H., (1994): Eine neue Methode zur Leistungsdiagnostik bei Tauchern. Wehrmed. M.sch., $\underline{38}(10)$, 326-329.

BRAUER, B.M./GOTTSCHALK ,K. (1996): Sportmedizin von A-Z. Heidelberg, Leipzig, Barth. 
BUSLAPS, C.; TETZLAFF, K.; SCHWARZKOPF, J.; NEUBAUER, B.; BETTINGHAUSEN, E.; RIECKERT, H. (1998): Vergleich der Herzkreislauffunktion sowie des Lactatverhaltens unter Normal-(0m) und Überdruck(30m). In Dt. Zeitschrift für Sportmed., 49(Sonderheft1), 64-67.

CHEN, A.A,; KENNY, G.P.; JOHNSTON, C.E.; GIESBRECHT, G.G. (1996): Design and evaluation of a modified underwater cycle ergometer. In: Can. J. Appl. Physiol, 21(4) 134-148.

COSTILL, D.L. (1971): Energy requirements during exercise in water. J Sports Med Phys Fitness 11(2), 87-92.

COUNSILMAN, J. (1980): Handbuch des Sportschwimmens. Bockenem.

DI PRAMPERO, P.E.; PENDERGAST, D.R.; WILSON, D.W.; RENNIE, D.W. (1974): Energetics of swimming in man. In: J Appl Physiol 37(1), 31-37.

DONALD, K.W.; DAVIDSON, W.M. (1954): Oxygen uptake of "botted" and 2fin swimming" divers. J Appl Physiol $\underline{7}$, 31-37.

DRÄGER; T. (1999): Sauerstoffaufnahme bei verschiedenen Schwimmgeschwindigkeiten mit Tauchgerät unter Berücksichtigung der Herzfrequenz. Unveröffentlichte Diplomarbeit, Deutsche Sporthochschule Köln.

DROSTE, T.; ALMELING, M.; WEGENER, R.; WITTEN, F.; HOTTOWITZ, R. (2001): Umgebungsdruckgesteuertes Exspirationsventil. Offenlegungsschrift DE 100 05843 A 1 beim Deutschen Patent- und Markenamt. 16.8.2001.

DU BOIS, DU BOIS (1916), Arch intern Med17, 863:

DWYER, J.; PILMANIS, A.A. (1978):Physiological Studies of Divers Working at Depths to $99 \mathrm{fsw}$ in the open Sea. In: Underwater Physiologie VI. Proceeding of the sixth symposium on underwater physiologie; Hrsg.: Shilling,C.W.; Beckett, M.W.; Federation of American Societies for Experimental Biology, Bethesda, 167-178.

DWYER, J. (1983): estimation of oxygen uptake from the heart rate response to undersea work. Undersea Biomed Res $\underline{10}$, 77-87.

EHM, O.F.; Hahn, M; Hoffmann, U.:(2003):Der neue Ehm, Tauchen noch sicherer!. Cham,

EUROPEAN DIVING TECHNOLOGY COMMITEE (2003): Fitness to Dive Standards. URL : www.edtc.org/EDTC-Fitnesstodivestandard-2003.pdf Zugriff am 16.8.2004

FOLEY; M.F.; BILLINGS, C.E.; HUIE, C.R. (1967): Development of techniques for direct measurement of metabolism under water. Aerosp Med $\underline{38}$, 153-155.

FOURTRIEB, VIER FLOSSENKONZEPTE IM VERGLEICH (2000). In: Ritter, H. (Hrsg.): unterwasser 5, 130-133. 
GOFF,L.B.; BRUBACH, H.F,; SPECHT, H. (1957): Measurements of respiratory responses and work efficiency of underwater swimmers utilizing improved instrumentation. In: J Appl Physiol 10(2), 197-202.

HASSEL, E.; LEDER, A. (2003):Einsatz der IT in der ingenieurwissenschaftlichen Grundlagenforschung zu umweltgerechten Produktgestaltung am Beispiel „Strömungs- und Verbrennungsmodellierung und -simulation“. Rostock. URL: http://www.uni-rostock.de/andere/wvu/Texte/abstract hassel.pdf . Zugriff am 22.7.2003

HELLWIG, M. (1997): Tauchen mit Querschnittsgelähmten. Göttingen.

HOFFMANN, U. (1995a): Methodisches Konzept der Tauchausbildung. In: HOFFMANN, U. (1995): Anfängerausbildung im Sporttauchen. Stuttgart.

HOFFMANN, U. (1995b): Sporttauchen. In Schwimmen Band 2 Hrsg. Kurt Wilke, Bockenem.

HOFFMANN, U. (2000):Schubwerkzeuge. In: tauchen 12/2000 Seite 123-132.

HOFFMANN, U. (2002): Leistungsphysiologische Forschungsansätze zum Flossenschwimmen, In: Deutsche Zeitschrift für Sportmedizin Juni 2002, 192195.

HOLMÉR, I. (1972): Oxygen uptake during swimming in man. Acta Physiol. Scand. Suppl. 407, 1-55.

HOLMÉR, I. (1974): Energy cost of arm stroke, leg kick and whole stroke in competitive swimming style. In; Eur. J. Appl. Physiol. 33,105-118.

HOLMÉR, I.; Astrand, P.O. (1972): Swimming training and maximal oxygen uptake. In: J. appl. Physiol., 33(4), 510-513.

HUBRAUM [JACKETTEST] (2000). In Ritter, H. (Hrsg.): unterwasser 3, 117-121.

JAHNS, N. (2000): Leistungsdiagnostische Untersuchungsmethoden für Sport- und Berufstaucher. unveröffentlichte Examensarbeit. Göttingen.

KARPOVICH, P.V.; PESTRECOV; K. (1939): Mechanical work and efficiency in swimming crawl and back strokes. In: Arbeitsphysiologie, Berlin, 10, 504-514.

KARPOVICH; P.V.; MILMAN, N. (1944): Energy expenditure in swimming. In: Am. J. Physiol. 142, 140-144.

KERLL, K.-H. (1994): Verband Deutscher Sporttaucher e.V. Chronik 1954-1994. Stuttgart.

KOSIAN; S.A. (2000): Herz-Kreislauf-Erkrankungen. In: ALMELING, M.; BÖHM, F.; WELSLAU, W. , 2002: Handbuch Tauch- und Hyperbarmedizin. Landsberg/Lech, II -7.4.3., 1-38.

KRASTEV, K.; PIRIOVA, B.; DOBREV, D.; BOGDANOV P. (1964): Gasaustausch beim Unterwasserschwimmen. Med Sport $\underline{4}, 77-80$. 
LATHAN, H.-H.; SCHUBERT, K. (1981): Ausgewählte sportmedizinische Beiträge zu Leistungsentwicklung in den Maximal- und Schnellkraftsportarten.

Studienmaterial Deutsche Hochschule für Körperkultur, Leipzig.

LETZELTER, M.(1978): Trainingsgrundlagen. Training-Technik-Taktik. Reinbek.

LÜCHTENBERG, D. (1988): ABC-Tauchen, Grundlagen, Fertigkeiten, Übungsformen. Konstanz.

MAGEL, J.R. (1971): Comparison of the physiologic response to varying intensities of submaximal work in tethered swimming and treadmill running. J Sports Med 11, 203-212.

MAGEL, J.R.; FAULKNER, J.A. (1969) Maximum oxygen uptake of college swimmers. J Appl Physiol 22(5), 929-933.

MARKER, F.; DURBEN,V.(1999): Strömungsmechanik, Vorlesungsskript, Wuppertal. URL: http://w3.uni-wuppertal.de/www/FB12/lg6/Literatur/stroemg.pdf ; Zugriff am 22.7.2003

MARTIN, D. (1980):Grundlagen der Trainingslehre Teil 2. Die Planung, Gestaltung und Steuerung des Trainings und das Kinder- und Jugendtraining. Schorndorf.

McARDLE, W.D.; GLASER, R.M.; MAGEL, R.M. (1971) Metabolic and cardiorespiratory response during free swimming and treadmill walking. J. Appl. Physiol. $\underline{30}(5)$, 733-738.

MARTIN, D. (1977): Grundlagen der Trainingslehre I. Schorndorf.

MARTIN, D. (1980): Grundlagen der Trainingslehre II. Schorndorf.

MEYER, D. (1972): Trainingstipps. Schwimmlage beim Flossenschwimmen. In: poseidon Jahrgang 1972 Heft 1-6 ohne Seitenangabe. Berlin

MONPETIT, R.R.; LÉGER, L.A.; LAVOIE, J.-M., CAZORLA, G. (1981): VO 2 peek during free swimming using backward extrpolation of the recovery curve. In: Eur. J. Appl. Physiol. 142, 385-391.

NEUBAUER, B.; TETZLAFF, K. (1999): Prospektive Lungenfunktionsmessung mit Hilfe eines elektronischen Miniatur-Spirometers zum Nachweis akuter obstruktiver Atemwegsveränderungen bei Tauchschülern während der beruflichen Tauchausbildung. Pneumonologie 53(4), 219-225.

NIKLAS, A. (1989): Entwicklungsergebnisse zur Ermittlung der aeroben Kraftausdauer mittels verschiedener Methoden den sportmedizinischen Spiroergometrie. Magdeburg.

NIKLAS, A.; HOTTOWITZ, R.; FUHRMANN, P.; WALTHER, G.; WELGER, K. (1982): Vorrichtung zur Parametererfassung im Schwimmen. Patentschrift (19) DD (11) 209688 B1, Amt für Erfindungs- und Patentwesen der Deutschen Demokratischen Republik. 19.8.1982. 
NIKLAS, A.; FUHRMANN, P.; HOTTOWITZ, R.; WALTHER, G.; WELGER, K. (1988): Verfahren und Vorrichtung zur Spiroergometrie im Wasser. Med Sport 28, 150-153.

NIKLAS; A.; PETER, E. (1993): Verfahren und Vorrichtung zur tätigkeitsspezifischen Leistungsdiagnostik für Schwimmtaucher. In: Bartmann, $\mathrm{H}$.: Taucherhandbuch, Landsberg/Lech VIII - 1.6, 99-106.

PENDERGAST, D.R.; DI PRAMPERO, P.E.; CRAIG, J.R.; WILSON, D.R.; RENNIE, D.W. (1977): Quantitativ analysis of the front crawl in men and women. In: J Appl Physiol 43(3), 475-479.

PENDERGAST, D.R.; TEDESCO; M.; NAWROCKI, D.M.; FISHER; N.M. (1996): Energetics of underwater swimming with SCUBA. In: Medicine and sience in sport and exercise 28(5), 573-580.

PENDERGAST, D.R.; MOLLENDORF, J.; LOGUE, C.; SAMIMY, S. (2003a): Evaluation of fins used in underwater swimming. In: UHM $\underline{30}, 57-73$.

PENDERGAST, D.R.; MOLLENDORF, J.; LOGUE, C.; SAMIMY, S. (2003b): Underwater fin swimming in women with reference to fin selection. In: UHM 30, 75-85.

PILMANIS, A.A.; HENRIKSEN, J.K.C.; DWYER, H.J. (1977): An Underwater Ergometer for Diver Work Performance Studies in the Ocean. Ergonomics $\underline{20}$, 51-55.

PROFESSIONAL ASSOCIATION OF DIVING INSTRUCTORS (PADI) (2001): Erklärung zum Gesundheitszustand. Form No. 10063G(Rev.09/01) Version 2.0. ohne Ort.

RUSSEL, C.J.; McNEILL, A.; EVONUK, E. (1972): Some cardiorespiratory and metabolic responses of Scuba divers to increased pressure and cold. Aeros Med 43(9), 998-1001.

RINGE, I.C.(2004): Med-Diss.Göttingen (in Vorbereitung). Mit freundlicher Genehmigung der Verfasserin.

RECREATIONAL SCUBA TRAINING COUNCIL (RSTC) (1997): facts and figures. Hettlingen.

SCHLICHTING, H.J. (1983): Energie und Energieentwertung in Naturwissenschaft und Umwelt. Heidelberg.

SCHNABEL, G, THIEß, G. (1993): Lexikon Sportwissenschaft. Berlin.

SCHEYER, W. (2001): Westen, Jackets und andere Auftriebskörper. In: Divemaster, Stuttgart 2/2001 S.19-24.

SCHLAG AUF SCHLAG (1997). In Jahr Verlag (Hrsg.): in tauchen 12, 98-108.

SCHUBWERKZEUGE (2000). In: Jahr Verlag(Hrsg.): tauchen 12, 122-132. 
SCHULZE, E. (1995): Tauchen. Hamburg.

SCUBA SCHOOLS INTERNATIONAL (SSI) (2002): Training \& Dealer Standards. ohne Ortsangabe.

SIMON, G.; THIESMANN, M.; FROHBERGER, U.; CLASING, D. (1983): Ergometrie im Wasser - eine neue Form der Leistungsdiagnostik beim Schwimmen. In: Dtsch. Z. Sportmed., 34(1), 5-14.

STEGEMANN, J. (1991): Leistungsphysiologie: physiologische Grundlagen der Arbeit und des Sports, Stuttgart, New York.

STEINBACH, K.; COEN, G.; SCHMITT W.M.; KINDERMANN, W. (1985) Wertigkeit der Fahrradergometrie zur Trainingssteuerung und Beurteilung der Leistungsfähigkeit bei Schwimmern und Schwimmerinnen. In : Franz, I.W.; Mellerowicz, H.; Noack, W.(Hrsg.): Training und Sport zur Prävention und Rehabilitation in der technisierten Umwelt. Berlin, Heidelberg 1985, 642-648.

STIBBE, A.(1997): Sporttauchen: der sichere Weg zum Tauchsport. 8. überarb. und erw. Auflage. Bielefeld, Stuttgart.

TOUSSAINT, H.M. (1988): Mechanics and energetics of swimming. Antwerpen.

TOUSSAINT, H.M.; BEELEN, A.; RODENBURG, A.; SARGEANT, A.J.; deGROOT, G.; HOLLANDER, A.P.; van INGEN SCHENAU, G.J. (1988): Propelling efficiency of front-crawl swimming. In: J Appl Physiol 65(6), 2506-2512.

ULMER, H.-V. (1983): Arbeitsphysiologie - Umweltphysiologie. In: Schmidt, R.F.; Thews, G.: Physiologie des Menschen. Berlin, Heidelberg, New York, 545566.

UNGERECHTS, B. (1978): Der Strömungswiderstand als Bewegungshemmender Faktor. In BAL(Hrsg) Informationen zum Training: Schwimmsport. In: Beihefte zum Leistungssport, Berlin 14, 58-71.

UNGERECHTS, B.; PERSYN, U.; COLEMAN,V. (2000): Analysis of Swimming Techniques using Vortex Traces. Leuven. URL http://www.coachesinfo.com/article/153/ Zugriff vom 13.2.2004

VERBAND DEUTSCHER SPORTTAUCHER e.V. (1987): Deutsche Tauchsportabzeichen - Abnahmekarte mit den Prüfungsbedingungen. Hamburg.

VERBAND DEUTSCHER SPORTTAUCHER e.V. (VDST) (2004):, Was ist Flossenschwimmen. Offenbach. URL: http://www.vdstflossenschwimmen.de/html/technik.html Zugriff am 11.6.2004.

WELSLAU, W. (1997): GTÜM-Ausschuß Tauchtauglichkeit. In: Caisson 12, 221-222.

WELTMAN;G.; ENGSTROM, G.H. (1969): Heart rate and respiratory response correlations in surface and underwater work. In: Aerospace Medicine 40(5), 479-483.

WENDLING, J.; EHM, O.; EHRSAM, R.; KNESSL, P.; NUSSBERGER, P. (2001): Tauchtauglichkeit Manual. Luzern. 
WESTEN, JACKETS UND ANDERE AUFTRIEBSKÖRPER (2001). In: MIT-PresS (Hrsg): Divemaster, Stuttgart 2 (34), 19- 24.

WITTEN, F. (2000): Blutdruckverhalten beim Tauchen. Dissertation. Göttingen.

ZAMPARO, P.; PENDERGAST, D. R.; TERMIN, B.; MINETTI, A. E. (2002): How fins affect the economy and efficiency of human swimming. In: J Exp Biol 205, 2665-2676 
8.1 Dokumente, Messreihen und Daten

8.1.1 Umgebungsdruckgesteuertes Exspirationsventil. Offenlegungsschrift

(19) BUNDESREPUBLIK DEUTSCHLAND

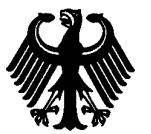

DEUTSCHES

PATENT- UND

MARKENAMT
(1) Offenlegungsschrift (1) DE 10005843 A 1
(21) Aktenzeichen:
(22) Anmeldetag:
(43) Offenlegungstag:
10005843.4
10. 2. 2000
16. 8.2001 |||||||||||||||||||||||||||||||||||||||||||||||||||||||||||||||||

(51) Int. Cl.7:

B 63 C 11/16

// A61B 5/08,A61M $39 / 22$
(71) Anmelder:

Droste, Thomas, 37077 Göttingen, DE; Almeling, Michael, Dr., 37085 Göttingen, DE; Wegener, Reinhold, 37079 Göttingen, DE; Witten, Fritz, 38440 Wolfsburg, DE; Hottowitz, Ralf, 39116 Magdeburg,
(12) Erfinder:

gleich Anmelder

Die folgenden Angaben sind den vom Anmelder eingereichten Unterlagen entnommen

(54) Umgebungsdruck gesteuertes Exspirationsventil

(57) Die Erfindung beinhaltet ein Exspirationsventil zur Atemgasanalyse von Tauchern. Dabei wird eine der beiden Öffnungen (z. B. 4) mit dem Ausgang eines Tauchautomaten und die andere Öffnung über einen Schlauch mit einem Gasanalysesystem verbunden. Das Ventil ist am Umfang mit zwei Lochreihen (3) versehen. Außderdem befindet sich im Inneren in kegelförmiger Außromuem körper (7) Krümmung aufurer Krummung aufweist und die beiden Teile (8) trennt. Umgeben ist der Ventilkörper mit einer elastischen, gummiartigen Membran (6).

Wirkungsweise:

Der Gasstrom gelangt durch die Öffnung (z. B. 4) auf den Strömungskörper (7), welcher den Gasstrom prallflächenfrei durch die Offnungen (3) auf die Membran (6) umlenkt. Diese Membran ist auf der Außenseite dem Umgebungsdruck ausgesetzt. Der Gasstrom kann an der Membran

- vorbei nur in die zweite Kammer (mit der Öffnung (z. B. 5))

- gelangen, wenn der Umgebungsdruck überwunden wird,

mann über einen Schlauch ein Gasanalysesystem ange-

schlossen werden.

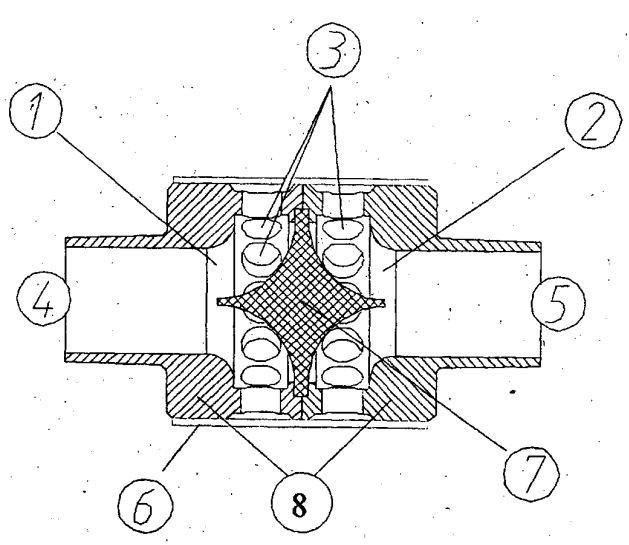

ᄂ2

웅

으

㟔 


\section{DE 10005843 A 1}

Beschreibung

Anwendungsgebiet der Erfindung

Die Erfindung findet Anwendung im Bereich Leistungs- 5 medizin, insbesondere bei spiroergometrischen Untersuchungen unter Wasser und für die Arbeits-, Sport- und Raumfahrtmedizin.

\section{Charakteristik der bekannten Lösungen}

Zur Gasanalyse einsetzbare Systeme, bei Tauchern, in gröBeren Tiefen (2. .10 m) wurde, bis auf (A61B/320155-7) keine adäquate Lösung gefunden. Dabei handelt es sich im wesentlichen um ein einstellbares federbelastetes Ventil. 1s Nachteilig ist, daß für jede Tauchtiefe der entsprechende Gegendruck manuell eingestellt werden muB.

\section{Ziel der Erfindung}

Ziel der Erfindung ist es, eine genaue Atemgasanalyse be der Spiroergometrie unter Wasser bei geringster Einschränkung des Tauchers zu ermöglichen.

\section{Darlegung des Wesens der Erfindung}

Der Erfindung liegt die Aufgabe zugrunde, eine genaue, Atemgasanalyse von Tauchern bei unterschiedlichen Tauchtiefen zu ermöglichen.

Erfindungsgemäß wird diese Aufgabe gelöst, indem cin 30 aus zwei Kammern bestehender, am Umfang jeder Kammer mit Öffnungen versehener vorzugsweise runder Körper mit einer gummiartigen Membran umgeben ist. ErfindungsgemäB ist weiterhin das die Kammern durch einen kegelformigen Körper voneinander getrennt sind. Weiterhin ist erfin- 35 dungsgemä $B$ daß, der kegelförmige Körper so gestaltet ist, $\mathrm{da} \beta$ ein vom Tauchautomaten einströmender Gasstrom prallflächenfrei durch die, am Umfang angebrachten Öffnungen auf die Gummimembran trifft. Des weiteren ist erfindungsgemäß, daß die Außenseite der Membran direkt 40 dem Wasserdruck ausgesetzt ist und das der Gasstrom vom Tauchautomaten, über die am Umfang angebrachten Öffnungen an der Membran vorbei in die zweite Kammer gelangt, wenn der Innendruck größer als der Wasserdruck ist. Erfindungsgemä $B$ wir die zweite Kammer über einen 45 Schlauch mit einem Gasanalysegerät verbunden.

\section{Ausführungsbeispiel}

Die Erfindung wird beispielhaft durch die anliegende 50 Zeichnung (Fig. 1) erläutert. Die eine als Stutzen ausgelegte Öffnung (im Beispiel (4)) wird mit der Ausatemseite eines Tauchautomaten verbunden und die andere, als Stutzen ausgelegte Öffnung (im Beispiel (5)) mit einem Gasanalysegerät verbunden, wobei eine Wirkungsumkehr möglich ist. Zur 55 Trennung der Kammern (1 u. 2) dient der Körper (7) welcher auf beiden Seiten kegelförmig ausgebildet ist. Am Umfang ist eine Membran (6) derart angebracht das die kleinen am Umfang angebrachten Öffnungen (3) bedeckt sind. Die elastische, gummiartige Membran (6) ist einerseits dem um- 60 gebendem Wasserdruck und zum anderen dem Ventilinnendruck ausgesetzt. Der auf beiden Seiten kegelförmige Körper (7) ist so gestaltet das eine Gasströmung prallfäächenfrei, durch die Öffnungen (3) auf die Membran (6) trifft. Im Falle eines, durch einen an die als Stutzen ausgelegte Öffnung 65 (z. B. 4) angeschlossenen Tauchautomaten realisierten, gröBeren Innendruckes gegenüber dem Umgebungsdruck kann der Gasstrom an der Membran (6) vorbei durch die Öffnun-
2

gen (3) in die zweite Kammer und von hier über die als Stutzen ausgelegte Öffnung (z. B. 5) über einen Schlauch zu einem Gasanalysegerăt gelangen.

\section{Patentansprüche}

1. Umgebungsdruckgesteuertes Exspirationsventil vorzugsweise zur Atemgasanalyse von Tauchern dadurch gekennzeichnet, $\mathrm{da} \beta$ ein aus zwei hintercinander angebrachten Kammern $(\mathbf{1}, 2)$ bestehender vorzugsweise runder am Umfang mit mehreren kleineren (3) und an den Stirnseiten mit zwei größeren, vorzugsweise als Stutzen ausgelegten Öffnungen $(4,5)$ versehener Körper (8), welcher mit einer flexiblen, vorzugsweise schlauchförmigen Membran (6) umgeben ist, welche die am Umfang angebrachten Öffnungen (3) bedeckt und an den äußeren Kanten des Körpers (8) dicht befestigt ist.

2. Umgebungsdruckgesteuertes Exspirationsventil vorzugsweise zur Atemgasanalyse nach Anspruch 1 dadurch gekennzeichnet, daß die eine größere stimseitig angebrachte Öffnung (4 oder 5) mit der Ausatmungsseite eines Tauchautomaten und die andere gröBere stirnseitig angebrachte Öffnung (5 oder 4 ) über ein flexibles Rohr, z. B. einen Schlauch mit einem Gasanalysesystem, oder einem Douglassack verbunden ist.

3. Umgebungsdruckgesteuertes Exspirationsventil vorzugsweise zur Atemgasanalyse nach Anspruch 1 und 2 dadurch gekennzeichnet, da 3 zur Vernngerung des Strömungswiderstandes die Kammern $(\mathbf{1}, \mathbf{2})$ durch einen, im Schnitt als Doppelkegel ausgelegten, vorzugsweise parabelförmigen Strömungskörper (7) getrennt sind.

4. Umgebungsdruckgesteuertes Exspirationsventil nach Anspruch 1 bis 3 dadurch gekennzeichnet, da $B$ mehrere umgebungsdruckgesteuerte Exspirationsventile parallel betrieben werden können.

Hierzu 1 Seite(n) Zeichnungen 


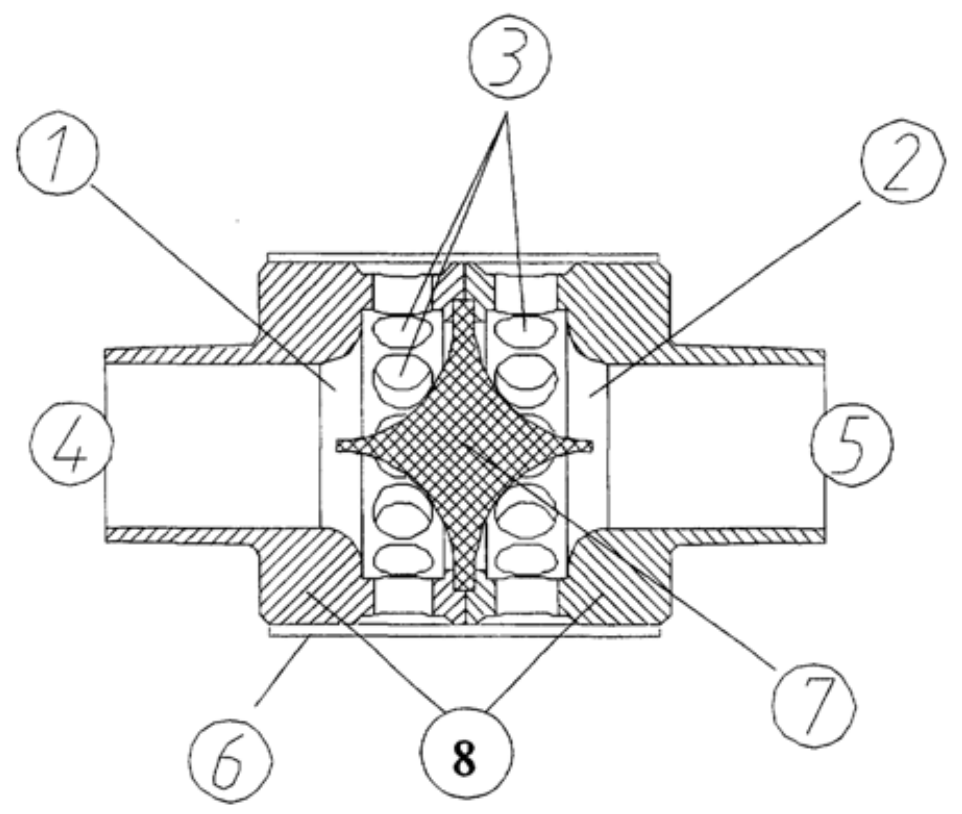

Figur 1 
8.1.2 Prüfprotokolle des Lungenautomaten mit umgebungsdruckgesteuerten Ausatemventil

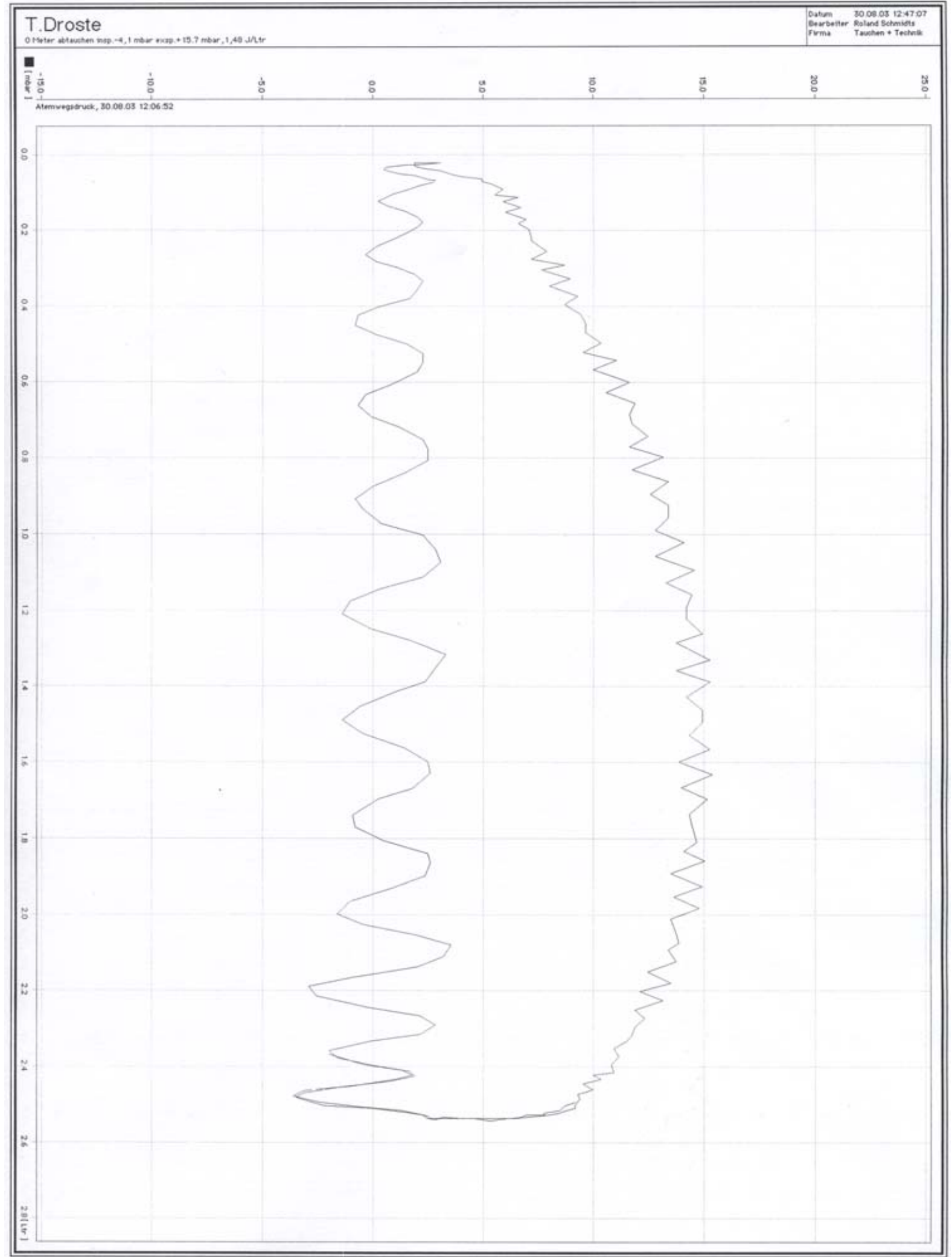

„0 m Tiefe Abtauchen 


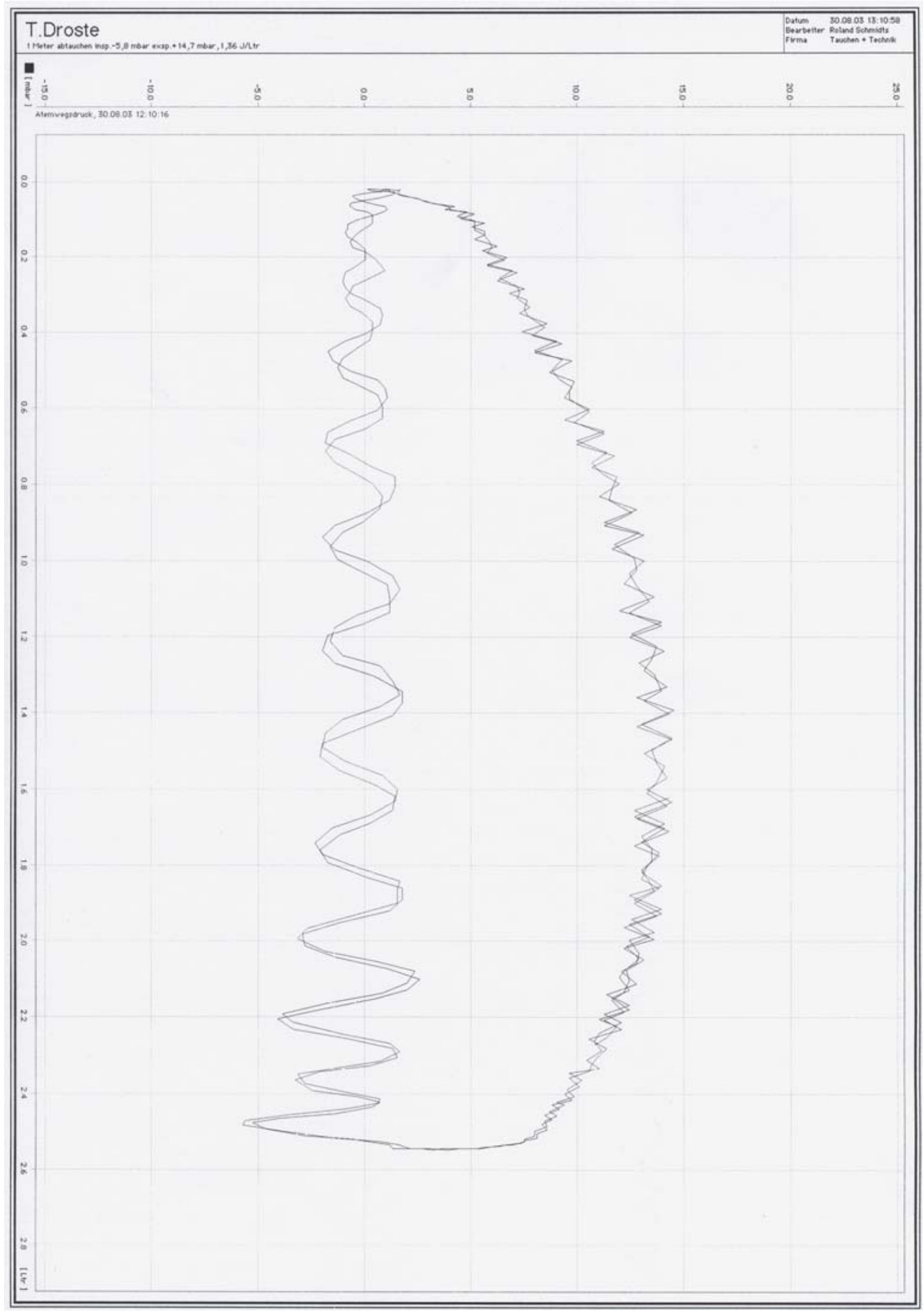

-1 m Abtauchen 


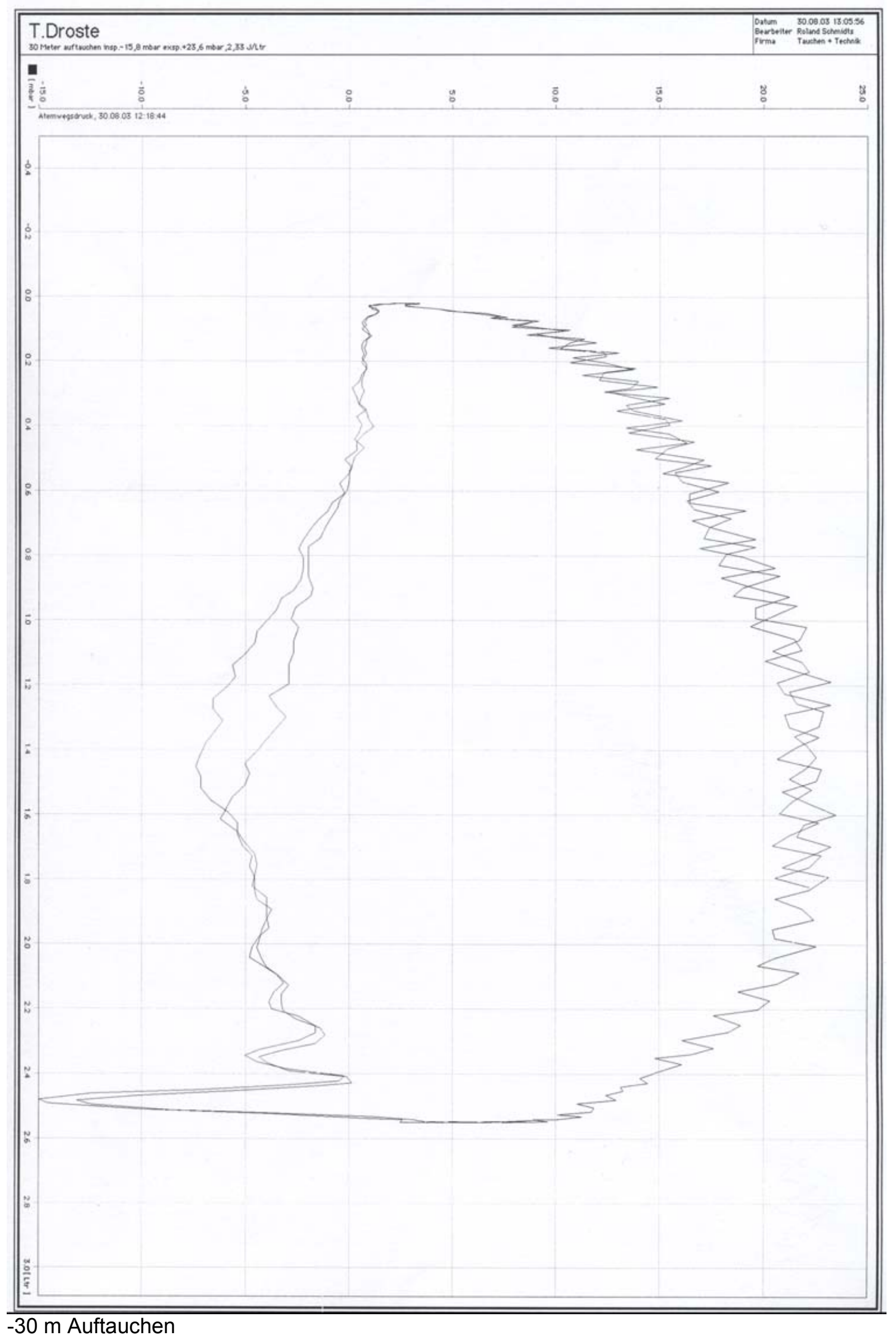




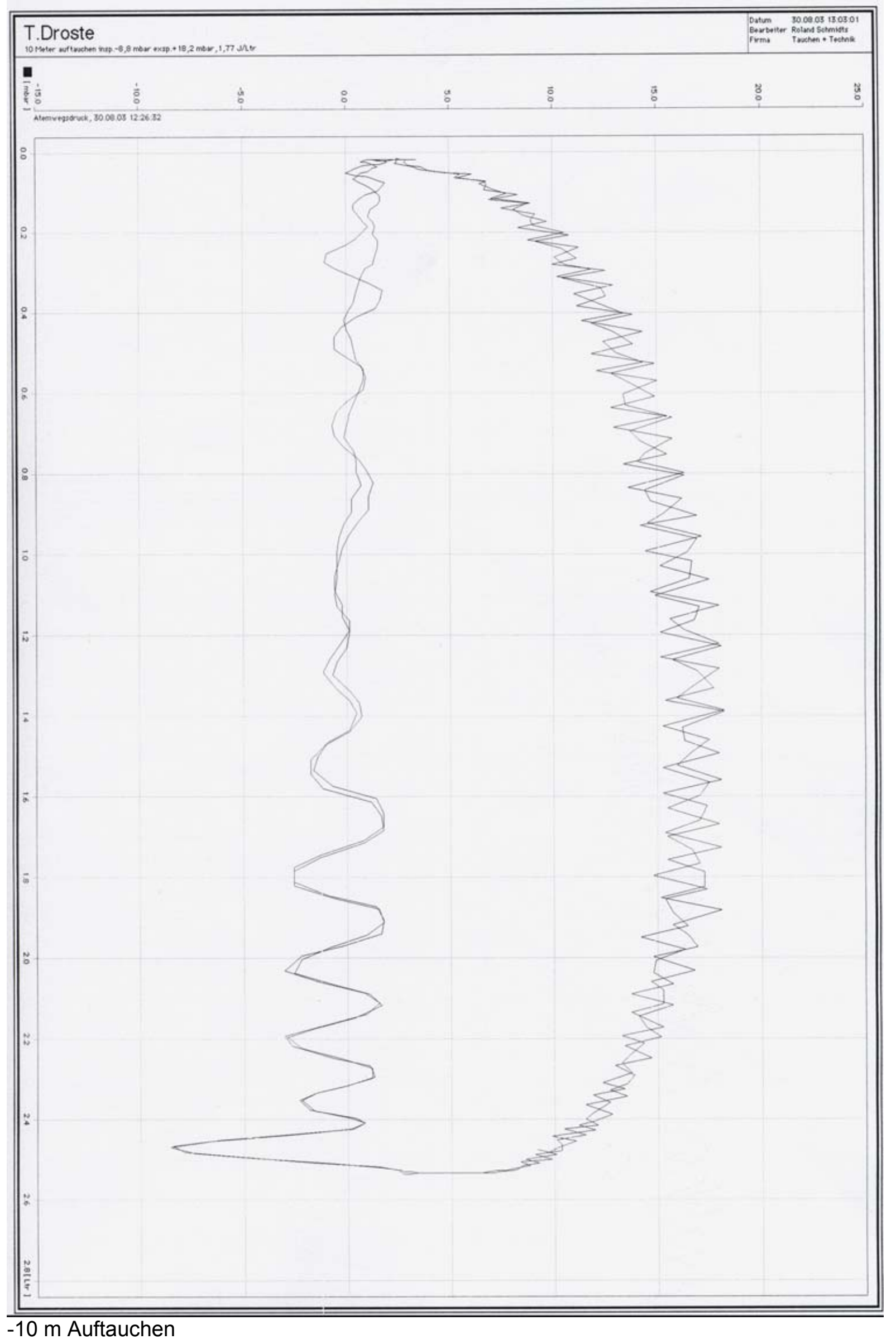




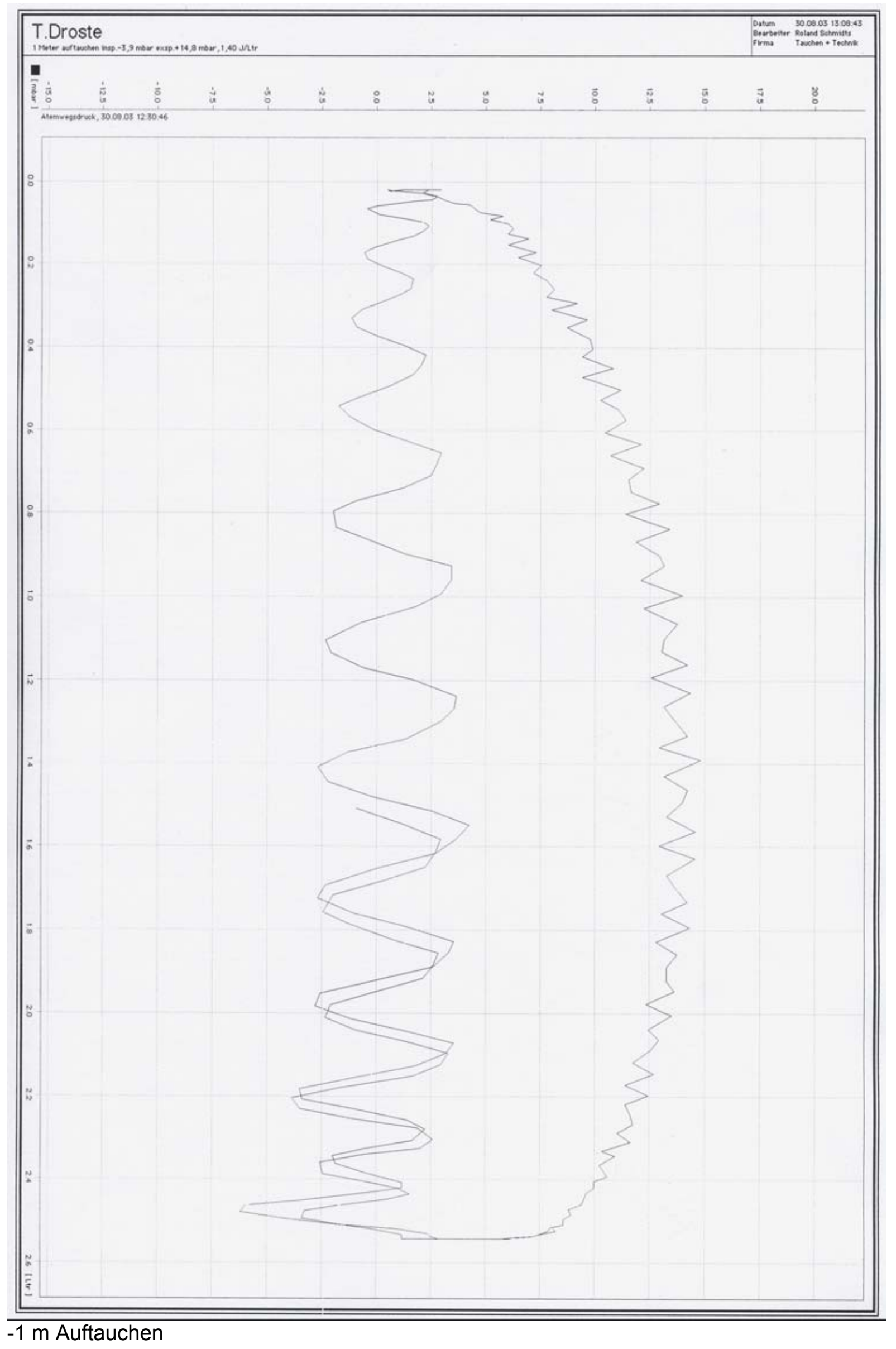




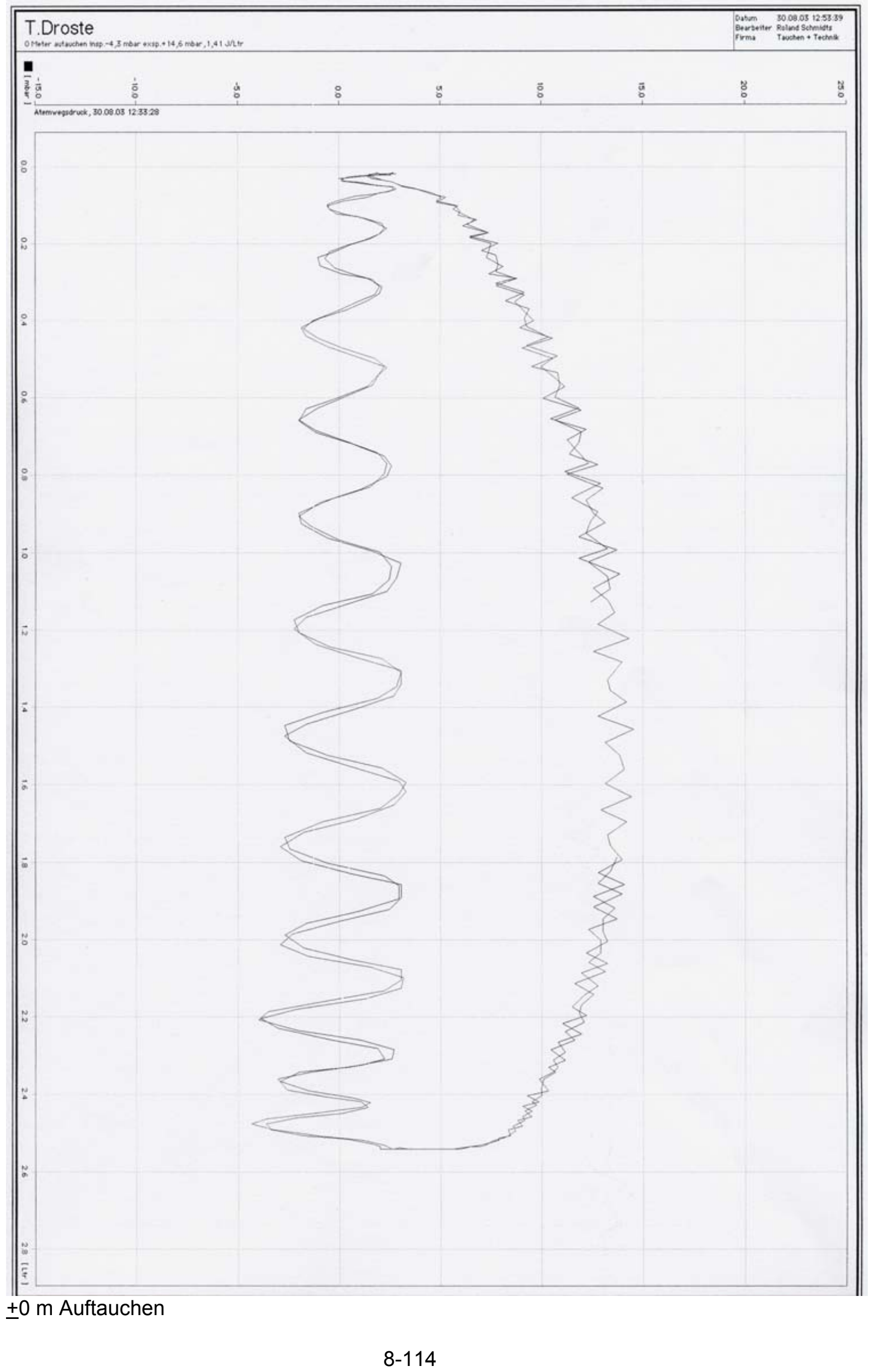




\subsubsection{Daten des Lungenautomaten Sherwood Brut}

Mit freundlicher Genehmigung der Fa. Balzer, Spessartstr. 13, D-36341 Lauterbach.

\section{SHERUOOD}

SCUBA

BALZER

\subsection{Technische Daten}

7.1. Technische Daten (allgemein)

Allgemeiner Hinweis:

Bei den unter Punkt 7.2 und 7.3 genannten Atemreglern sind fol-

gende technische Details gleich:

Bauart:

Downstream-System, kolbengesteuert kompensiert (außer Brut)

zum Teil ausgestattet mit Wärmeleitmetall (Blizzard und Maximus)

und/oder Feuchtigkeitsspeicher (Oasis und Maximus)

Druckminderer (1. Stufe) mit CBS-System

Betriebsdruck $200 \mathrm{bar}$

Durchflussrate „CBS-System" $13-25 \mathrm{ccm} / \mathrm{min}$

Gehäuse CDA-360 Messing verchromt

O-Ringe Buna- $\mathrm{N}^{\circledast}$

CBS-Ventil Polyäthylen-Propylen

Kolbensitz Teflon ${ }^{\circledR}$
Dosiereinrichtung (2. Stufe)

Schlauchlänge

O-Ringe

Gehäuse

Gehäuseabdeckung

Ventilsitz

Membrane

Ausatemmembrane

Mundstück
$790 \mathrm{~mm}$ (Maximus $1.000 \mathrm{~mm}$ )

Buna- $N^{8}$

Triax

Thermoplastik-Vinyl

Polymer (geeignet für niedrige

Temperaturen)

Tufel $^{8}$

Thermoplastik Elastomer

Wisdom Mundstück auf

Liquid-Silikon-Basis

(bei Brut und Oktopus-

Systemen C-Flex)

Die Zertifizierung nach den Richtlinien 89/686/EWG des Rates erfolgte durch:

SGS Yarsley International Certification Service Limited

SGS House, Portland Road

East Grimstead, West Sussex, RH 19 4ET

England

Amtlich registrierte Prüf-Einrichtung Nr.: 0120.

Sherwood-Atemregler erfüllen die Norm nach EN 250, wurden auf einer Tiefe von $50 \mathrm{~m}$ zertifiziert und sind kaltwassertauglich.

7.2. Technische Daten (Atemregler)

\begin{tabular}{|l|c|c|c|c|c|c|}
\hline & Maximus & Blizzard & Oasis & Magnum & Brut \\
\hline Luftlieferleistung (bei atmosphärischem Druck von 1 bar) & $935 \mathrm{l} / \mathrm{min}$ & $935 \mathrm{l} / \mathrm{min}$ & $935 \mathrm{l} / \mathrm{min}$ & $935 \mathrm{l} / \mathrm{min}$ & $850 \mathrm{l} / \mathrm{min}$ \\
\hline Einatemwiderstand (bei atmosphärischem Druck von $1 \mathrm{bar}$ ) & $28 \mathrm{~mm}$ WS & $28 \mathrm{~mm}$ WS & $28 \mathrm{~mm}$ WS & $28 \mathrm{~mm}$ WS & $25 \mathrm{~mm}$ WS \\
\hline Ausatemwiderstand (bei atmosphärischem Druck von 1 bar) & $18 \mathrm{~mm}$ WS & $18 \mathrm{~mm}$ WS & $18 \mathrm{~mm}$ WS & $18 \mathrm{~mm}$ WS & $18 \mathrm{~mm}$ WS \\
\hline
\end{tabular}

\begin{tabular}{|c|c|c|c|c|c|}
\hline \multicolumn{6}{|l|}{ Druckminderer (1. Stufe) } \\
\hline Gewicht & $820 \mathrm{~g}$ & $820 \mathrm{~g}$ & $820 \mathrm{~g}$ & $770 \mathrm{~g}$ & $770 \mathrm{~g}$ \\
\hline Mitteldruck & $8,5-10,5$ bar & $8,5-10,5$ bar & $8,5-10,5$ bar & $8,5-10,5$ bar & $8,5-10,5$ bar \\
\hline Optimale Einstellung & 9,5 bar & $9,5 \mathrm{bar}$ & 9,5 bar & $9,5 \mathrm{bar}$ & 9,5 bar \\
\hline Niederdruckanschlüsse (3/8"-24 UNF) & 5 & 5 & 5 & 4 & 3 \\
\hline Hochdruckanschlüsse (7/16"-20 UNF) & 2 & 2 & 2 & 2 & 1 \\
\hline Balancierte 1. Stufe & - & - & - & - & - \\
\hline Integrierter Vereisungsschutz & - & - & - & $\cdot$ & - \\
\hline Patentiertes CBS.Ventil (Constant Bleed System) & - & - & - & - & - \\
\hline Luftsensor-Kanal (ASC-System) & 2 & 2 & 2 & 2 & 1 \\
\hline M.O.B.System (Moving Orifice Balance) & - & - & - & - & - \\
\hline umrüstbar auf 300 bar & • & - & - & - & - \\
\hline
\end{tabular}




\begin{tabular}{|c|c|c|c|c|c|}
\hline & Maximus & Blizzard & Oasis & Magnum & Brut \\
\hline \multicolumn{6}{|l|}{ Dosiereinrichtung (2. Stufe) } \\
\hline Gewicht (ohne Mitteldruckschlauch) & $250 \mathrm{~g}$ & $160 \mathrm{~g}$ & $160 \mathrm{~g}$ & $150 \mathrm{~g}$ & $150 \mathrm{~g}$ \\
\hline Balanciert & - & $\cdot$ & $\cdot$ & - & - \\
\hline Patentierter Wärmespeicher der Ausatemluft & - & $\cdot$ & -- & -- & - \\
\hline Patentierter Feuchtigkeitsspeicher der Ausatemluft & $\bullet$ & -- & - & - & -- \\
\hline Atemwiderstand einstellbar & $\cdot$ & -- & -- & -- & -- \\
\hline Deflektor & $\cdot$ & -- & -- & - & - \\
\hline Wisdom-Mundstück & 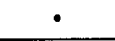 & $\stackrel{\bullet}{ }$ & $\cdot$ & - & -- \\
\hline
\end{tabular}

\section{SHERWOOD}

7.3. Technische Daten (Oktopus-Systeme)

\begin{tabular}{|c|c|c|}
\hline & Minimus & Oktopus \\
\hline $\begin{array}{l}\text { Einatemwiderstand } \\
\text { (bei atmosphärischem Druck } \\
\text { von } 1 \text { bar) }\end{array}$ & $52 \mathrm{~mm}$ WS & $28 \mathrm{~mm}$ WS \\
\hline $\begin{array}{l}\text { Ausatemwiderstand } \\
\text { (bei atmosphärischem Druck } \\
\text { von } 1 \text { bar) }\end{array}$ & $13 \mathrm{~mm}$ WS & $13 \mathrm{~mm}$ WS \\
\hline Gewicht & $125 \mathrm{~g}$ & $150 \mathrm{~g}$ \\
\hline Balanciert & - & $\bullet$ \\
\hline Wisdom-Mundstück & -- & - \\
\hline
\end{tabular}

7.4. Zertifiziert nach EN 250

Alle Sherwood-Atemregler sind von einer unabhängigen Prüfinstitution auf der Grundlage der Richtlinie 89/686 EWG und der Europäschen Norm EN 250 getestet und zertifiziert worden.

Diese Zertifizierung nach EN 250 betrifft die nachstehend aufg führten Atemreglermodelle und Oktopus-Systeme:

$\begin{array}{lllll}\text { Modell } & & \begin{array}{r}\text { Warmwassertest } \\ \text { (über } 10^{\circ} \mathrm{C} \text { ) }\end{array} & \begin{array}{c}\text { Kaltwassertest } \\ \text { (unter } 10^{\circ} \mathrm{C} \text { ) }\end{array} \\ \text { SRB } & 5100 & \text { Brut } & \text { zertifziert } & \text { zertifiziert } \\ \text { SRB } & 5300 & \text { Magnum } & \text { zertifziert } & \text { zertifiziert } \\ \text { SRB } & 5600 & \text { Maximus } & \text { zertifziert } & \text { zertifiziert } \\ \text { SRB } & 5700 & \text { Oasis } & \text { zertifziert } & \text { zertifiziert } \\ \text { SRB } & 5900 & \text { Blizzard } & \text { zertifzięrt } & \text { zertifiziert } \\ & & & & \\ \text { SR } & 3107 & \text { Oktopus } & \text { zertifziert } & \text { zertifiziert } \\ \text { SR } & 3108 & \text { Minimus } & \text { zertifziert } & \text { zertifiziert }\end{array}$




\subsubsection{Mittelwerte, Standardabweichung und Konfidenzintervall der an den Messflügeln erhobenen Werte (Messreihe 1)}

\begin{tabular}{|l|r|r|r|r|r|r|}
\hline Messtiefe & \multicolumn{4}{|l|}{$8 \mathrm{~mm}$ bei 5 Messflügeln } & & \\
\hline & & & & & & \\
\hline $\mathrm{v}_{\text {eingestell }} /\left(\mathrm{m} \cdot \mathrm{s}^{-1}\right)$ & 0,3 & 0,6 & 0,8 & 0,9 & 1,2 & 1,5 \\
\hline Mittelwert $\mathrm{v}_{\text {gemessen }}$ & 0,4034 & 0,6681 & 0,8384 & 0,9492 & 1,3028 & 1,6640 \\
\hline Standardabweichung & 0,0207 & 0,0425 & 0,0523 & 0,0696 & 0,0894 & 0,0982 \\
\hline Konfidenzintervall & 0,0006 & 0,0013 & 0,0016 & 0,0022 & 0,0028 & 0,0030 \\
\hline
\end{tabular}

\begin{tabular}{|l|r|r|r|r|r|r|}
\hline Messtiefe & \multicolumn{4}{|l|}{$80 \mathrm{~mm}$ bei 3 Messflügeln } & & \\
\hline & & & & & & \\
\hline $\mathrm{v}_{\text {eingestellt }} /\left(\mathrm{m} \cdot \mathrm{s}^{-1}\right)$ & 0,3 & 0,6 & 0,8 & 0,9 & 1,2 & 1,5 \\
\hline Mittelwert $\mathrm{v}_{\text {gemessen }}$ & 0,4020 & 0,6610 & 0,8328 & 0,9432 & 1,3017 & 1,6538 \\
\hline Standardabweichung & 0,0155 & 0,0343 & 0,0415 & 0,0527 & 0,0790 & 0,0997 \\
\hline Konfidenzintervall & 0,0006 & 0,0014 & 0,0017 & 0,0021 & 0,0032 & 0,0040 \\
\hline
\end{tabular}

\begin{tabular}{|l|r|r|r|r|r|r|}
\hline Messtiefe & \multicolumn{4}{|l|}{$470 \mathrm{~mm}$ bei 5 Messflügeln } & & \\
\hline & & & & & & \\
\hline $\mathrm{v}_{\text {eingestellt }} /\left(\mathrm{m} \cdot \mathrm{s}^{-1}\right)$ & 0,3 & 0,6 & 0,8 & 0,9 & 1,2 & 1,5 \\
\hline Mittelwert $\mathrm{v}_{\text {gemessen }}$ & 0,3684 & 0,6022 & 0,7549 & 0,8547 & 1,1588 & 1,4282 \\
\hline Standardabweichung & 0,0337 & 0,0476 & 0,0603 & 0,0643 & 0,0749 & 0,0700 \\
\hline Konfidenzintervall & 0,0010 & 0,0015 & 0,0019 & 0,0020 & 0,0024 & 0,0034 \\
\hline
\end{tabular}

\begin{tabular}{|l|r|r|r|r|r|r|}
\hline Messtiefe & \multicolumn{4}{|l|}{ 470 $\mathrm{mm}$ bei 3 Messflügeln } & & \\
\hline & & & & & & \\
\hline $\mathrm{V}_{\text {eingestellt }} /\left(\mathrm{m} \cdot \mathrm{s}^{-1}\right)$ & 0,3 & 0,6 & 0,8 & 0,9 & 1,2 & 1,5 \\
\hline Mittelwert $\mathrm{v}_{\text {gemessen }}$ & $\mathbf{0 , 3 5 6 7}$ & $\mathbf{0 , 5 8 1 7}$ & $\mathbf{0 , 7 2 8 8}$ & $\mathbf{0 , 8 2 8 9}$ & $\mathbf{1 , 1 2 8 3}$ & $\mathbf{1 , 3 9 5 2}$ \\
\hline Standardabweichung & 0,0181 & 0,0229 & 0,0248 & 0,0275 & 0,0354 & 0,0415 \\
\hline Konfidenzintervall & 0,0007 & 0,0009 & 0,0010 & 0,0011 & 0,0015 & 0,0026 \\
\hline
\end{tabular}

\begin{tabular}{|c|c|c|c|c|c|c|}
\hline Messtiefe & \multicolumn{4}{|c|}{$80 \mathrm{~mm}$ und $470 \mathrm{~mm}$ bei 5 Messflügeln } & & \\
\hline$V_{\text {eingestellt }} /\left(m \cdot s^{-1}\right)$ & 0,3 & 0,6 & 0,8 & 0,9 & 1,2 & 1.5 \\
\hline Mittelwert $\mathrm{v}_{\text {gemessen }}$ & 0,3858 & 0,6417 & 0,7967 & 0,9019 & 1,2344 & 1,5955 \\
\hline Standardabweichung & 0,0330 & 0,0551 & 0,0702 & 0,0820 & 0,1096 & 0,1404 \\
\hline Konfidenzintervall & 0,0007 & 0,0012 & 0,0015 & 0,0018 & 0,0025 & 0,0037 \\
\hline
\end{tabular}

\begin{tabular}{|c|c|c|c|c|c|c|}
\hline \begin{tabular}{|l|} 
Messtiefe \\
\end{tabular} & \multicolumn{4}{|c|}{$80 \mathrm{~mm}$ und $470 \mathrm{~mm}$ bei 3 Messflügeln } & & \\
\hline $\mathrm{V}_{\text {eingestellt }} /\left(\mathrm{m} \cdot \mathrm{s}^{-1}\right)$ & 0,3 & 0,6 & 0,8 & 0,9 & 1,2 & 1,5 \\
\hline Mittelwert $v_{\text {gemessen }}$ & 0,3791 & 0,6293 & 0,7808 & 0,8860 & 1,2193 & 1,5787 \\
\hline Standardabweichung & 0,0283 & 0,0492 & 0,0622 & 0,0709 & 0,1066 & 0,1461 \\
\hline Konfidenzintervall & 0,0008 & 0,0014 & 0,0018 & 0,0020 & 0,0031 & 0,0049 \\
\hline
\end{tabular}


8.1.5 Abstand zwischen Mittelwert und Median in den Schleppversuchen mit unterschiedlichen Auftriebskörpern (Messreihe 2)

\begin{tabular}{|l|c|c|c|c|c|}
\hline \multicolumn{7}{|c|}{ Abstand zwischen Mittelwert und Median je Messreine } \\
\hline Proband A & $\begin{array}{l}0,3567 \\
\mathrm{~m} \cdot \mathrm{s}^{-1}\end{array}$ & $\begin{array}{c}0,5817 \\
\mathrm{~m} \cdot \mathrm{s}^{-1}\end{array}$ & $\begin{array}{c}0,8289 \\
\mathrm{~m} \cdot \mathrm{s}^{-1}\end{array}$ & $\begin{array}{c}1,1283 \\
\mathrm{~m} \cdot \mathrm{s}^{-1}\end{array}$ & $\begin{array}{c}1,3951 \\
\mathrm{~m} \cdot \mathrm{s}^{-1}\end{array}$ \\
\hline Auftriebskörper & & & & & \\
\hline HUB & 0,0113 & 0,3402 & 0,5925 & 0,6216 & 0,0000 \\
\hline Jacket & 0,2890 & 0,1459 & $-0,0963$ & 1,1006 & 0,2730 \\
\hline Kragenweste & $-0,0014$ & $-0,0072$ & 0,1562 & 0,3316 & $-0,3071$ \\
\hline Wing Jacket & 0,0611 & $-0,1488$ & 0,2349 & 0,2612 & 0,4340 \\
\hline
\end{tabular}

\begin{tabular}{|l|c|c|c|c|c|}
\hline \multicolumn{7}{|c|}{ Abstand zwischen Mittelwert und Median je Messreihe } \\
\hline Proband B & $\begin{array}{c}0,3567 \\
\mathrm{~m} \cdot \mathrm{s}^{-1}\end{array}$ & $\begin{array}{c}0,5817 \\
\mathrm{~m} \cdot \mathrm{s}^{-1}\end{array}$ & $\begin{array}{c}0,8289 \\
\mathrm{~m} \cdot \mathrm{s}^{-1}\end{array}$ & $\begin{array}{c}1,1283 \\
\mathrm{~m} \cdot \mathrm{s}^{-1}\end{array}$ & $\begin{array}{c}1,3951 \\
\mathrm{~m} \cdot \mathrm{s}^{-1}\end{array}$ \\
\hline Auftriebskörper & & & & & \\
\hline HUB & 0,0151 & $-0,3104$ & $-0,0218$ & 0,0323 & $-0,0219$ \\
\hline Jacket & 0,3240 & 0,1287 & 0,5997 & $-0,1427$ & 0,4730 \\
\hline Kragenweste & $-0,5892$ & $-0,0663$ & 0,6174 & 0,1614 & 0,7075 \\
\hline Wing Jacket & 0,4372 & 0,2055 & 0,6589 & 0,3126 & 0,5366 \\
\hline
\end{tabular}

8.1.6 Die Schiefe der Verteilung der Kraftwerte $F_{D p}$ von Proband A und B mit unterschiedlichen Auftriebskörpern (Messreihe 2)

\begin{tabular}{|l|c|c|c|c|c|}
\hline \multicolumn{1}{|c|}{ Proband A } & $\begin{array}{c}0,3567 \\
\mathrm{~m} \cdot \mathrm{s}^{-1}\end{array}$ & $\begin{array}{c}0,5817 \\
\mathrm{~m} \cdot \mathrm{s}^{-1}\end{array}$ & $\begin{array}{c}0,8289 \\
\mathrm{~m} \cdot \mathrm{s}^{-1}\end{array}$ & $\begin{array}{c}1,1283 \\
\mathrm{~m} \cdot \mathrm{s}^{-1}\end{array}$ & $\begin{array}{c}1,3951 \\
\mathrm{~m} \cdot \mathrm{s}^{-1}\end{array}$ \\
\hline Auftriebskörper & & & & & \\
\hline HUB & $-0,182$ & 0,624 & 0,408 & 0,291 & 0,280 \\
\hline Jacket & 0,336 & 0,317 & 0,210 & 0,464 & 0,041 \\
\hline Kragenweste & 0,184 & 0,248 & 0,292 & $-0,006$ & 0,032 \\
\hline Wing Jacket & 0,318 & 0,105 & 0,340 & 0,155 & 0,157 \\
\hline
\end{tabular}

\begin{tabular}{|l|c|c|c|c|c|}
\hline \multicolumn{1}{|c|}{ Proband B } & $\begin{array}{c}0,3567 \\
\mathrm{~m} \cdot \mathrm{s}^{-1}\end{array}$ & $\begin{array}{c}0,5817 \\
\mathrm{~m} \cdot \mathrm{s}^{-1}\end{array}$ & $\begin{array}{c}0,8289 \\
\mathrm{~m} \cdot \mathrm{s}^{-1}\end{array}$ & $\begin{array}{c}1,1283 \\
\mathrm{~m} \cdot \mathrm{s}^{-1}\end{array}$ & $\begin{array}{c}1,3951 \\
\mathrm{~m} \cdot \mathrm{s}^{-1}\end{array}$ \\
\hline Auftriebskörper & & & & & \\
\hline HUB & $-0,126$ & 0,254 & 0,231 & 0,476 & $-0,002$ \\
\hline Jacket & 0,083 & 0,427 & 0,208 & $-0,003$ & 0,387 \\
\hline Kragenweste & $-0,305$ & 0,170 & 0,265 & 0,408 & 0,327 \\
\hline Wing Jacket & $-0,314$ & 0,167 & 0,415 & 0,201 & $\mathbf{0 , 4 9 8}$ \\
\hline
\end{tabular}




\subsubsection{Die Verteilung der größten und der kleinsten Schiefe je Proband (Messreihe 2)}

Die Diagramme stellen die im Anhang 8.1.6 farbig hervorgehobenen Werte dar.
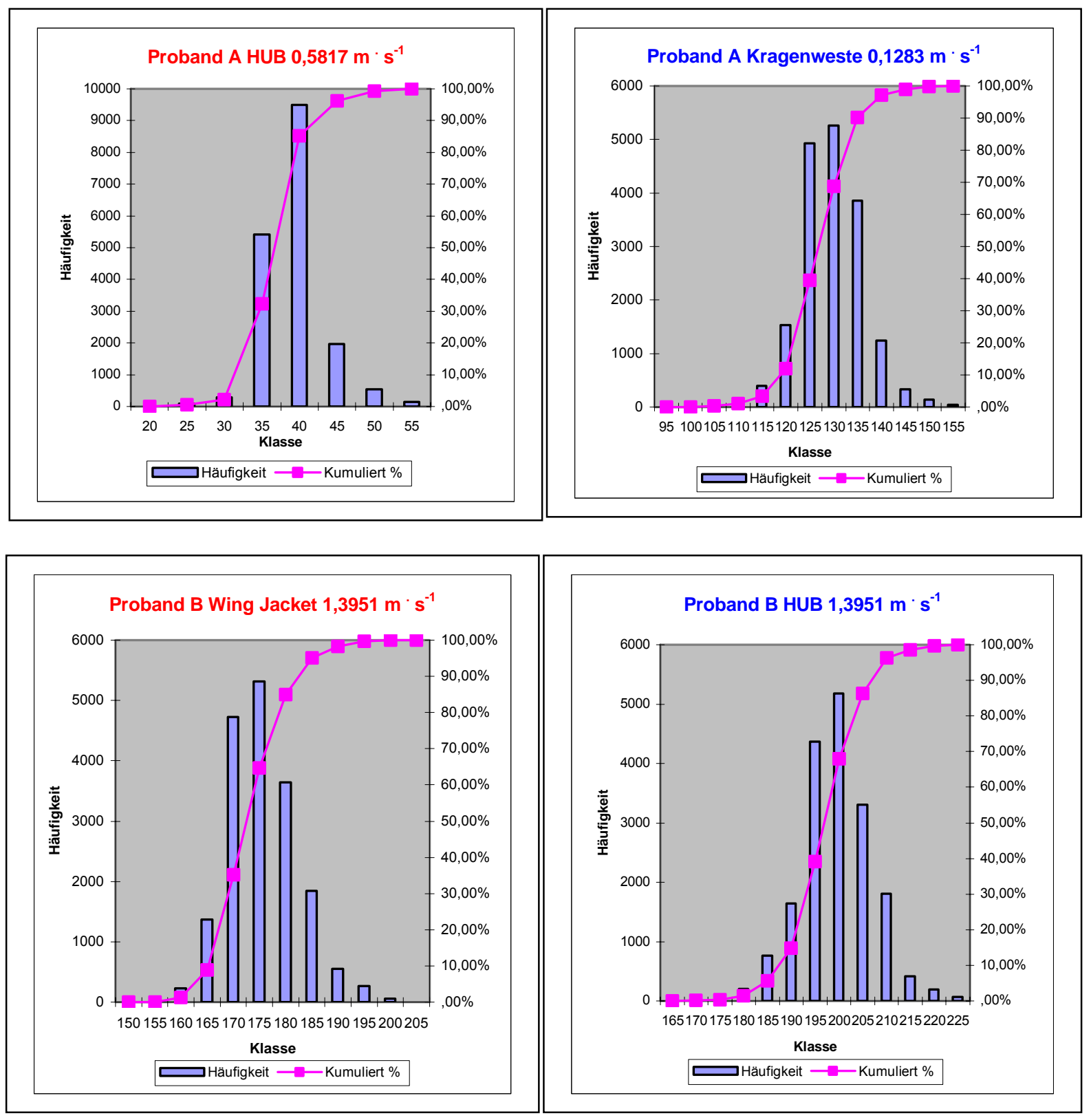
8.1.8 Abstand zwischen Mittelwert und Median in den Schleppversuchen mit unterschiedlichen Flossen (Messreihe 3)

\begin{tabular}{|l|c|c|c|c|c|}
\hline \multicolumn{7}{|c|}{ Abstand zwischen Mittelwert und Median je Messreihe } \\
\hline Proband A & $\begin{array}{c}0,3567 \\
\mathrm{~m} \cdot \mathrm{s}^{-1}\end{array}$ & $\begin{array}{c}0,5817 \\
\mathrm{~m} \cdot \mathrm{s}^{-1}\end{array}$ & $\begin{array}{c}0,8289 \\
\mathrm{~m} \cdot \mathrm{s}^{-1}\end{array}$ & $\begin{array}{c}1,1283 \\
\mathrm{~m} \cdot \mathrm{s}^{-1}\end{array}$ & $\begin{array}{c}1,3951 \\
\mathrm{~m} \cdot \mathrm{s}^{-1}\end{array}$ \\
\hline Flosse & & & & & \\
\hline Mares Volo & 0,4999 & 0,0966 & 0,2595 & 0,5182 & 0,0000 \\
\hline Sporasub Dessault & 0,7959 & 0,2927 & $-0,0707$ & 0,4606 & 0,0553 \\
\hline Seemann Sub SF 2 & 0,7378 & 0,4457 & 0,0987 & 0,1506 & 0,1115 \\
\hline Monoflosse & 1,1953 & 2,8914 & 0,9658 & 0,2877 & 0,3969 \\
\hline
\end{tabular}

\begin{tabular}{|l|c|c|c|c|c|}
\hline \multicolumn{7}{|c|}{ Abstand zwischen Mittelwert und Median je Messreine } \\
\hline Proband B & $\begin{array}{c}0,3567 \\
\mathrm{~m} \cdot \mathrm{s}^{-1}\end{array}$ & $\begin{array}{c}0,5817 \\
\mathrm{~m} \cdot \mathrm{s}^{-1}\end{array}$ & $\begin{array}{c}0,8289 \\
\mathrm{~m} \cdot \mathrm{s}^{-1}\end{array}$ & $\begin{array}{c}1,1283 \\
\mathrm{~m} \cdot \mathrm{s}^{-1}\end{array}$ & $\begin{array}{c}1,3951 \\
\mathrm{~m} \cdot \mathrm{s}^{-1}\end{array}$ \\
\hline Flosse & & & & & \\
\hline Mares Volo & 0,1151 & 1,0404 & 0,5730 & 0,1987 & $-0,2047$ \\
\hline Sporasub Dessault & $-0,0245$ & $-0,1357$ & $-0,0417$ & 0,1389 & 0,5450 \\
\hline Seemann Sub SF 2 & $-0,0787$ & 0,3208 & 0,3601 & 0,2588 & $-0,1746$ \\
\hline Monoflosse & 0,8327 & 1,1984 & 1,3619 & 0,8003 & $-0,3532$ \\
\hline
\end{tabular}

8.1.9 Die Schiefe der Verteilung der Kraftwerte $F_{D p}$ von Proband A und B mit unterschiedlichen Flossen (Messreihe 3)

\begin{tabular}{|l|c|c|c|c|c|}
\hline \multicolumn{1}{|c|}{ Proband A } & $\begin{array}{c}0,3567 \\
\mathrm{~m} \cdot \mathrm{s}^{-1}\end{array}$ & $\begin{array}{c}0,5817 \\
\mathrm{~m} \cdot \mathrm{s}^{-1}\end{array}$ & $\begin{array}{c}0,8289 \\
\mathrm{~m} \cdot \mathrm{s}^{-1}\end{array}$ & $\begin{array}{c}1,1283 \\
\mathrm{~m} \cdot \mathrm{s}^{-1}\end{array}$ & $\begin{array}{c}1,3951 \\
\mathrm{~m} \cdot \mathrm{s}^{-1}\end{array}$ \\
\hline Flosse & & & & & \\
\hline Mares Volo & 0,496 & 0,232 & $-0,033$ & $-0,235$ & 0,335 \\
\hline Sporasub Dessault & 0,574 & 0,347 & $-0,019$ & 0,130 & $\mathbf{0 , 0 1 0}$ \\
\hline Seemann Sub SF 2 & 0,567 & 0,416 & 0,265 & 0,234 & 0,054 \\
\hline Monoflosse & 0,467 & $\mathbf{0 , 8 1 1}$ & 0,724 & 0,293 & 0,398 \\
\hline
\end{tabular}

\begin{tabular}{|l|c|c|c|c|c|}
\hline \multicolumn{1}{|c|}{ Proband B } & $\begin{array}{c}0,3567 \\
\mathrm{~m} \cdot \mathrm{s}^{-1}\end{array}$ & $\begin{array}{c}0,5817 \\
\mathrm{~m} \cdot \mathrm{s}^{-1}\end{array}$ & $\begin{array}{c}0,8289 \\
\mathrm{~m} \cdot \mathrm{s}^{-1}\end{array}$ & $\begin{array}{c}1,1283 \\
\mathrm{~m} \cdot \mathrm{s}^{-1}\end{array}$ & $\begin{array}{c}1,3951 \\
\mathrm{~m} \cdot \mathrm{s}^{-1}\end{array}$ \\
\hline Flosse & & & & & \\
\hline Mares Volo & 0,524 & 0,6422 & 0,183 & 0,243 & $\mathbf{0 , 0 3 5}$ \\
\hline Sporasub Dessault & 0,528 & 0,159 & 0,294 & 0,072 & 0,232 \\
\hline Seemann Sub SF 2 & 0,704 & 0,604 & 0,142 & 0,310 & 0,156 \\
\hline Monoflosse & $\mathbf{1 , 0 6 3}$ & 0,871 & 0,967 & 0,703 & $-0,271$ \\
\hline
\end{tabular}




\subsubsection{Die Verteilung der größten und der kleinsten Schiefe je Proband (Messreihe 3)}

Die Diagramme stellen die im Anhang 8.1.9 farbig hervorgehobenen Werte dar.

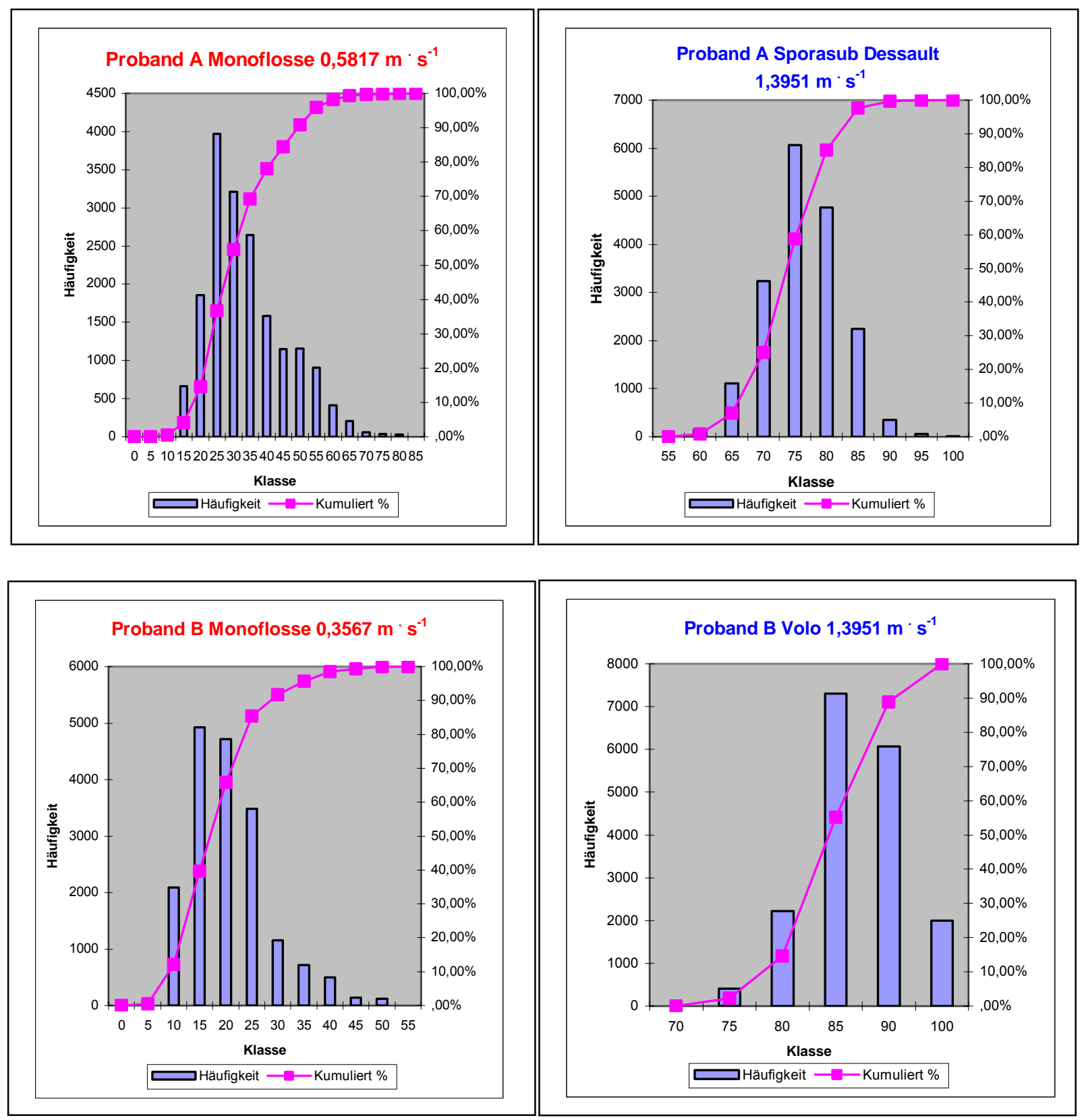




\subsubsection{Messkurven aller Versuche (Messreihe 4)}
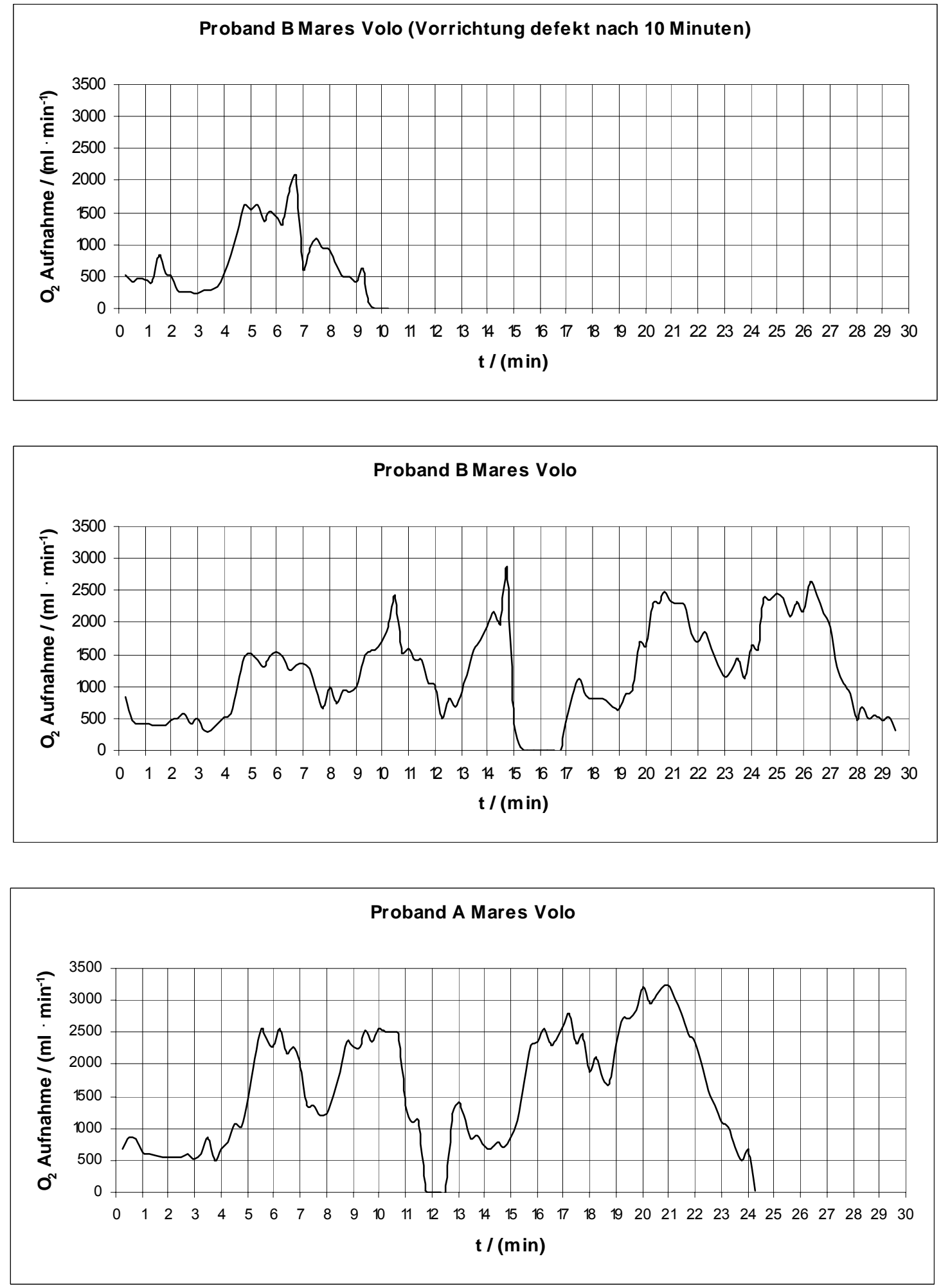

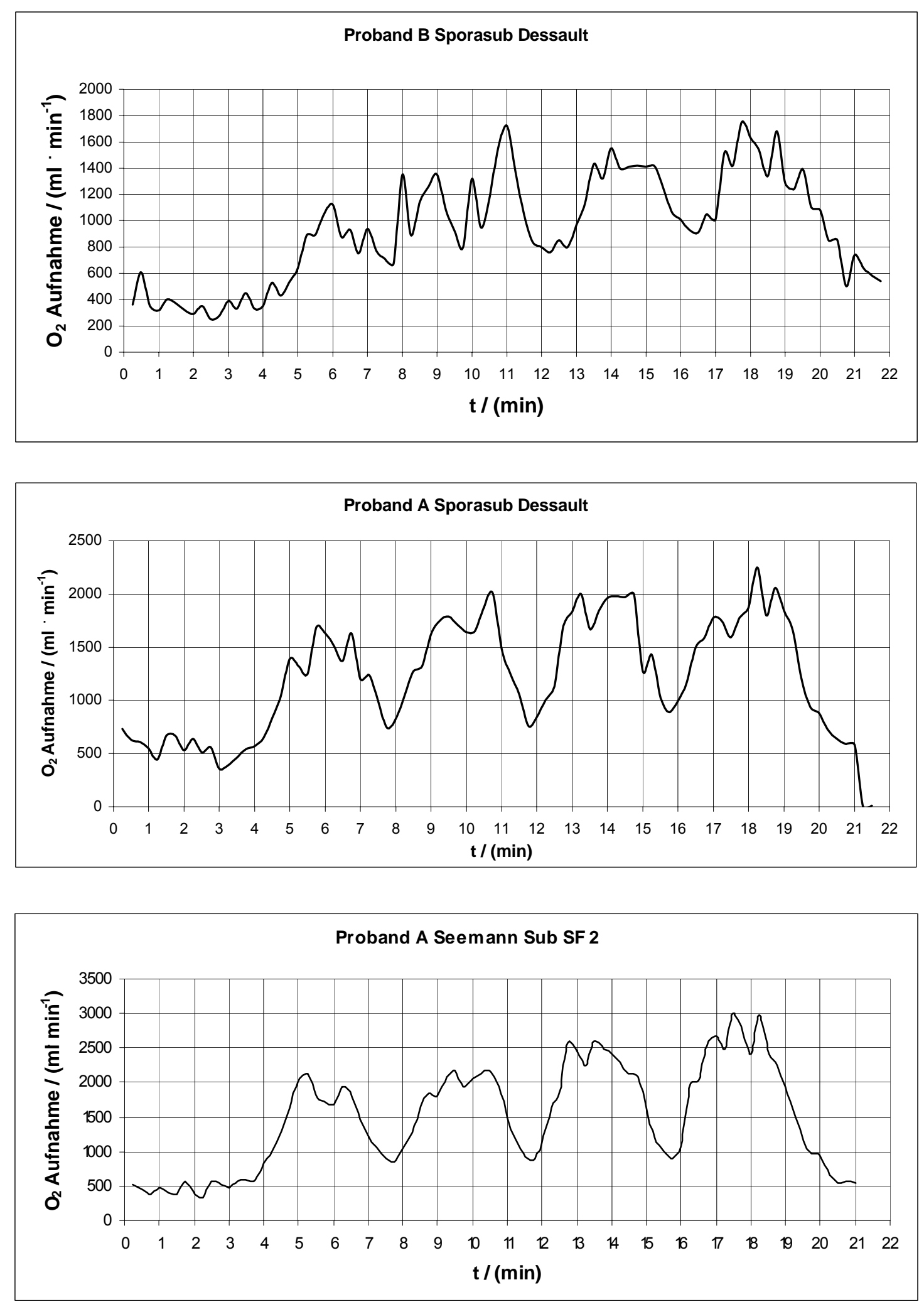

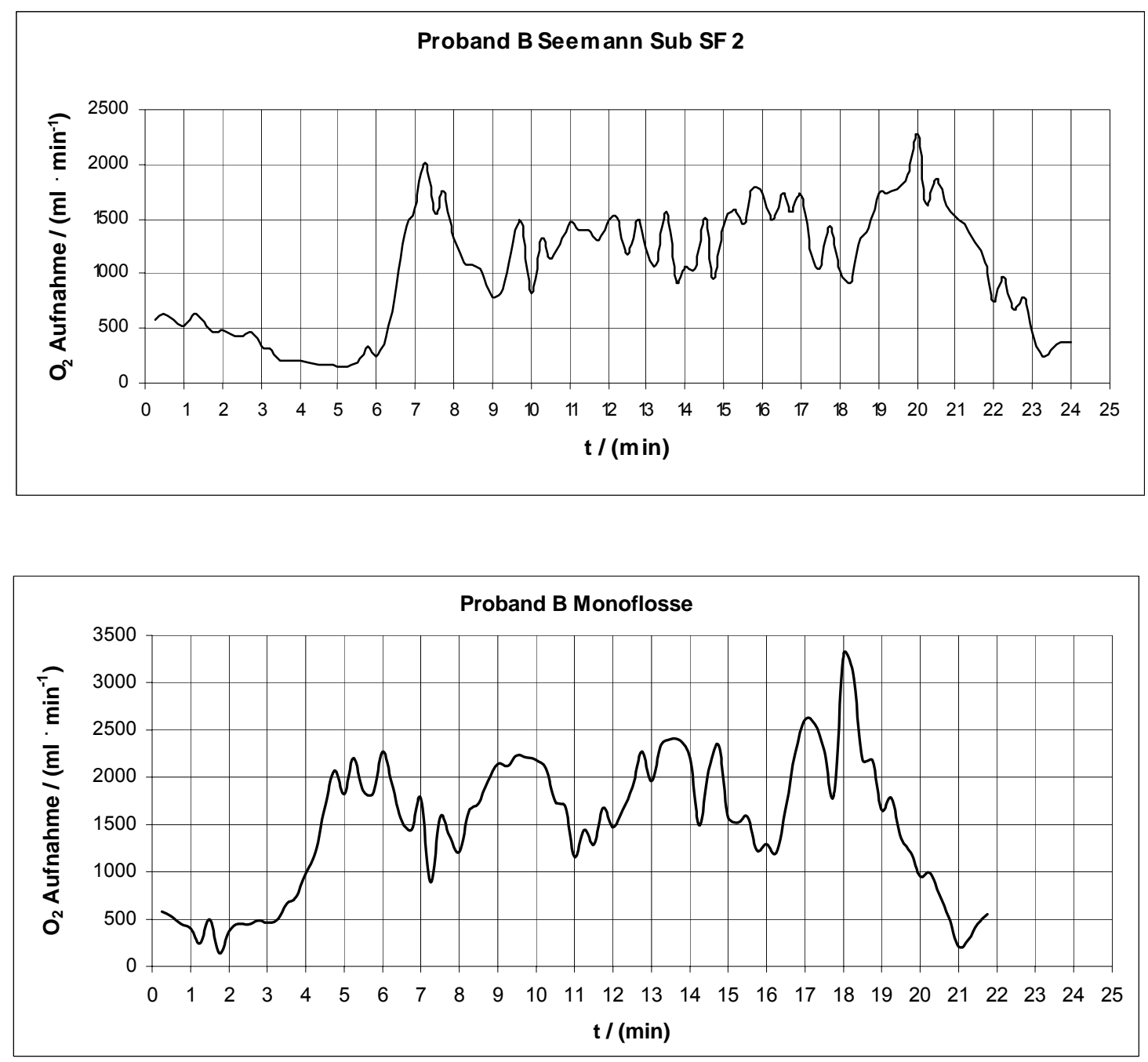


\subsubsection{Proband A. Messdaten (Messreihe 4)}

\begin{tabular}{|c|c|c|c|c|c|c|c|c|c|}
\hline \multicolumn{10}{|c|}{ Proband A } \\
\hline & \multicolumn{2}{|c|}{ Mares Volo } & & \multicolumn{3}{|c|}{ Seemann Sub SF 2} & \multicolumn{3}{|c|}{ Sporasub Dessault } \\
\hline & \multicolumn{3}{|c|}{$\mathrm{v}=0,7288 \mathrm{~m} \cdot \mathrm{s}^{-1}$} & \multicolumn{3}{|c|}{$\mathrm{v}=0,7288 \mathrm{~m} \cdot \mathrm{s}^{-1}$} & \multicolumn{3}{|c|}{$v=0,7288 \mathrm{~m} \cdot \mathrm{s}^{-1}$} \\
\hline Zeit & $\mathrm{VO}_{2}$ & $\mathrm{VCO}_{2}$ & $\mathrm{RQ}$ & $\mathrm{VO}_{2}$ & $\mathrm{VCO}_{2}$ & $\mathrm{RQ}$ & $\mathrm{VO}_{2}$ & $\mathrm{VCO}_{2}$ & \\
\hline$(\min )$ & $(\mathrm{ml}$ & $\left.\min ^{-1}\right)$ & & $(\mathrm{ml} \cdot \mathrm{r}$ & $\left.\min ^{-1}\right)$ & & $(\mathrm{ml} \cdot \mathrm{l}$ & $\left.\min ^{-1}\right)$ & \\
\hline & & & & & 40 & & & 80 & \\
\hline 0 & & & 1,2 & & 30 & 1,244 & & 90 & 1,4 \\
\hline 0,75 & & 1100 & 1,325 & & 470 & 1,27 & & 90 & 1,45 \\
\hline 1 & 630 & 880 & 1,397 & 480 & 590 & 1,229 & & 820 & 1,49 \\
\hline 1,25 & & 880 & 1,467 & & 460 & 1,15 & & & \\
\hline 1,5 & & 820 & 1,439 & 380 & 20 & 1,105 & & 60 & 1,43 \\
\hline 1,75 & & 00 & 1,45 & & & 1,07 & & & \\
\hline 2 & & 0 & 1,393 & & & 1,051 & & 00 & 1,50 \\
\hline 2,25 & 54 & 740 & 1,37 & 33 & 40 & 1,03 & & & 1, \\
\hline 2 & & & 1,32 & & & 1,018 & & & \\
\hline 2,75 & & 90 & 1,339 & & 20 & 0,981 & & 30 & 1,482 \\
\hline 3 & 530 & 710 & 1,34 & 480 & 460 & 0,958 & & 520 & 1,44 \\
\hline 3,25 & & 0 & 1,322 & & & 0,947 & & & \\
\hline 3,5 & 870 & 1140 & 1,31 & & 70 & 0,95 & & 90 & 1,28 \\
\hline 3,75 & & 30 & 347 & & & 0,95 & & 70 & \\
\hline 4 & & & & & & 0,94 & & 60 & 1, \\
\hline 4,25 & & 40 & 0,821 & 100 & 50 & 0,85 & & 80 & 1,04 \\
\hline 4,5 & & & & 1 & & 777 & & & 0,88 \\
\hline 4,75 & 102 & 30 & 0,667 & 1640 & 1220 & 0,744 & 040 & 90 & 0,7 \\
\hline 5 & 1460 & 20 &, 63 & 2000 & 1490 & 0,745 & 1390 & 980 & 0,70 \\
\hline 5,25 & 210 & 00 & 619 & 21 & 70 & 0,784 & 13 & 10 & 0,68 \\
\hline 5,5 & 2560 & 1680 & 0,656 & 183 & 1500 & 0,82 & 1250 & 840 & 0,67 \\
\hline 5,75 & 2410 & & 0,722 & 17 & & 0,85 & 1690 & & \\
\hline 6 & 2260 & 750 & 774 & 167 & 460 & 0,874 & 30 & 100 & 0,67 \\
\hline 6,25 & 2560 & 2050 & 0,801 & 195 & 1720 & 0,882 & 1510 & 1050 & 0,6 \\
\hline 6,5 & 216 & & & 18 & & 904 & & 90 & 0,72 \\
\hline 6,75 & 2260 & 1940 & 0,858 & 1460 & 1360 & 0,932 & 1630 & 1200 & 0,73 \\
\hline 7 & 2030 & 1880 & 0,926 & 1240 & & 0,984 & 1200 & 930 & 0,77 \\
\hline 7,25 & 135 & 1390 & 03 & 1070 & 1100 & 1,028 & 1240 & 1040 & 0,83 \\
\hline 7,5 & 1360 & 1530 & 1,125 & & 980 & 1,089 & 990 & 890 & $0,8 \mathrm{~s}$ \\
\hline 7,75 & 1210 & & & 860 & 970 & 1,1 & 40 & 700 & 0,94 \\
\hline 8 & 1230 & 1310 & 065 & 1050 & 1150 & 1,095 & 830 & 820 & 0,98 \\
\hline 8,25 & 1510 & 1370 & 0,907 & 1270 & 1290 & 1,016 & 1050 & 970 & 0.9 \\
\hline 8,5 & 1890 & 1580 & 836 & 1660 & 1560 & 0,94 & 1270 & 1070 & 0,84 \\
\hline 8,75 & 2340 & 1930 & 0,825 & 1850 & 1680 & 0,908 & 1320 & 1070 & 0,81 \\
\hline 9 & 2280 & 1910 & 0,838 & 1790 & 1630 & 0,911 & 1620 & 1280 & 0,7 \\
\hline 9,25 & 2280 & 1970 & 0,864 & 2090 & 1890 & 0,904 & 1750 & 1370 & 0,78 \\
\hline 9,5 & 2540 & 2220 & 0,874 & 2180 & 1990 & 0,913 & 1790 & 1410 & $0,7 \varepsilon$ \\
\hline 9,75 & 2340 & & & 195 & 1820 & 0,933 & & 1370 & \\
\hline 10 & 2570 & 2310 & 0,899 & 2060 & 1900 & 0,922 & 1640 & 1340 & 0,81 \\
\hline
\end{tabular}




\begin{tabular}{|c|c|c|c|c|c|c|c|c|c|}
\hline \multicolumn{10}{|c|}{ Proband A } \\
\hline & Mares & Volo & & \multirow{2}{*}{\multicolumn{3}{|c|}{$\begin{array}{l}\text { Seemann Sub SF } 2 \\
v=0,7288 \mathrm{~m} \cdot \mathrm{s}^{-1}\end{array}$}} & \multicolumn{3}{|c|}{ Sporasub Dessault } \\
\hline & \multicolumn{3}{|c|}{$\mathrm{v}=0,7288 \mathrm{~m} \cdot \mathrm{s}^{-1}$} & & & & $v=0,7$ & $288 \mathrm{~m} \cdot$ & $s^{-1}$ \\
\hline Zeit & $\mathrm{VO}_{2}$ & $\mathrm{VCO}_{2}$ & $\mathrm{RQ}$ & $\mathrm{VO}_{2}$ & $\mathrm{VCO}_{2}$ & $\mathrm{RQ}$ & $\mathrm{VO}_{2}$ & \begin{tabular}{|l|l}
$\mathrm{VCO}_{2}$ & $\mathrm{~F}$ \\
\end{tabular} & $\mathrm{RQ}$ \\
\hline$(\min )$ & $(\mathrm{ml}$ & $\left.\min ^{-1}\right)$ & & $(\mathrm{ml} \cdot \mathrm{r}$ & $\left.\min ^{-1}\right)$ & & $\left(\mathrm{ml}^{\prime} \mathrm{l}\right.$ & $\left.\min ^{-1}\right)$ & \\
\hline 10,25 & 2510 & 2280 & 0,908 & 2130 & 1970 & 0,925 & 1650 & 1350 & 0,818 \\
\hline 10,5 & 2500 & 2290 & 0,916 & 2170 & 2030 & 0,935 & 1870 & 1560 & 0,834 \\
\hline 10,75 & 2480 & 2380 & 0,96 & 1950 & 1890 & 0,969 & 2010 & 1730 & 0,861 \\
\hline 11 & 1360 & 1470 & 1,081 & 1500 & 1580 & 1,053 & 1490 & 1380 & 0,926 \\
\hline 11,25 & 1110 & 1310 & 1,18 & 1190 & 1360 & 1,143 & 1250 & 1260 & 1,008 \\
\hline 11,5 & 1120 & 1370 & 1,223 & 930 & 1130 & 1,215 & 1050 & 1140 & 1,086 \\
\hline 11,75 & 20 & 20 & 1 & 880 & 1110 & 1,261 & 760 & 870 & 1,145 \\
\hline 12 & 10 & 10 & 1 & 1110 & 1300 & 1,171 & 840 & 1000 & 1,19 \\
\hline 12,25 & 0 & 0 & & 1620 & 1620 & 1 & 1000 & 1100 & 1,1 \\
\hline 12,5 & 10 & 10 & 1 & 1880 & 1690 & 0,899 & 1130 & 1110 & 0,982 \\
\hline 12,75 & 1220 & 1490 & 1,221 & 2570 & 2230 & 0,868 & 1700 & 1450 & 0,853 \\
\hline 13 & 1400 & 1660 & 1,186 & 2430 & 2170 & 0,893 & 1830 & 1490 & 0,814 \\
\hline 13,25 & 1200 & 1430 & 1,192 & 2240 & 2080 & 0,929 & 2000 & 1640 & 0,82 \\
\hline 13,5 & 830 & 1020 & 1,229 & 2600 & 2460 & 0,946 & 1670 & 1410 & 0,844 \\
\hline 13,75 & 900 & 1140 & 1,267 & 2490 & 2400 & 0,964 & 1840 & 1580 & 0,859 \\
\hline 14 & 730 & 940 & 1,288 & 2410 & 2380 & 0,988 & 1960 & 1670 & 0,852 \\
\hline 14,25 & 690 & 920 & 1,333 & 2300 & 2300 & 1 & 1980 & 1700 & 0,859 \\
\hline 14,5 & 780 & 1040 & 1,333 & 2130 & 2130 & 1 & 1970 & 1730 & 0,878 \\
\hline 14,75 & 700 & 940 & 1,343 & 2080 & 2090 & 1,005 & 2000 & 1800 & 0,9 \\
\hline 15 & 860 & 1080 & 1,256 & 1640 & 1770 & 1,079 & 1270 & 1210 & 0,953 \\
\hline 15,25 & 1130 & 1120 & 0,991 & 1200 & 1400 & 1,167 & 1430 & 1420 & 0,993 \\
\hline 15,5 & 1720 & 1380 & 0,802 & 990 & 1230 & 1,242 & 1020 & 1070 & 1,049 \\
\hline 15,75 & 2310 & 1740 & 0,753 & 910 & 1170 & 1,286 & 890 & 970 & 1,09 \\
\hline 16 & 2340 & 1800 & 0,769 & 1070 & 1280 & 1,196 & 990 & 1080 & 1,091 \\
\hline 16,25 & 2560 & 2000 & 0,781 & 1990 & 1960 & 0,985 & 1170 & 1170 & 1 \\
\hline 16,5 & 2290 & 1870 & 0,817 & 2030 & 1800 & 0,887 & 1500 & 1340 & 0,893 \\
\hline 16,75 & 2440 & 2040 & 0,836 & 2530 & 2190 & 0,866 & 1590 & 1360 & 0,855 \\
\hline 17 & 2590 & 2220 & 0,857 & 2680 & 2330 & 0,869 & 1780 & 1510 & 0,848 \\
\hline 17,25 & 2790 & 2450 & 0,878 & 2480 & 2200 & 0,887 & 1740 & 1480 & 0,851 \\
\hline 17,5 & 2330 & 2110 & 0,906 & 3010 & 2750 & 0,914 & 1590 & 1380 & 0,868 \\
\hline 17,75 & 2450 & 2200 & 0,898 & 2730 & 2650 & 0,971 & 1780 & 1540 & 0,865 \\
\hline 18 & 1890 & 1760 & 0,931 & 2410 & 2400 & 0,996 & 1870 & 1640 & 0,877 \\
\hline 18,25 & 2110 & 2100 & 0,995 & 2990 & 2890 & 0,967 & 2250 & 1990 & 0,884 \\
\hline 18,5 & 1780 & 1910 & 1,073 & 2410 & 2370 & 0,983 & 1800 & 1630 & 0,906 \\
\hline 18,75 & 1710 & 1770 & 1,035 & 2240 & 2300 & 1,027 & 2060 & 1880 & 0,913 \\
\hline 19 & 2330 & 2160 & 0,927 & 1940 & 2120 & 1,093 & 1840 & 1750 & 0,951 \\
\hline 19,25 & 2720 & 2380 & 0,875 & 1510 & 1830 & 1,212 & 1650 & 1710 & 1,036 \\
\hline 19,5 & 2720 & 2380 & 0,875 & 1160 & 1540 & 1,328 & 1210 & 1380 & 1,14 \\
\hline 19,75 & 2840 & 2540 & 0,894 & 970 & 1360 & 1,402 & 940 & 1140 & 1,213 \\
\hline 20 & 3220 & 2920 & 0,907 & 940 & 1360 & 1,447 & 880 & 1100 & 1,25 \\
\hline 20,25 & 2950 & 2760 & 0,936 & 670 & 1010 & 1,507 & 720 & 910 & 1,264 \\
\hline 20,5 & 3070 & 2880 & 0,938 & 550 & 850 & 1,545 & 640 & 820 & 1,281 \\
\hline
\end{tabular}




\begin{tabular}{|c|c|c|c|c|c|c|c|c|c|}
\hline \multicolumn{10}{|c|}{ Proband A } \\
\hline & \multirow{2}{*}{\multicolumn{3}{|c|}{$\mathrm{v}=0,7288 \mathrm{~m} \cdot \mathrm{s}^{-1}$}} & \multirow{2}{*}{\multicolumn{3}{|c|}{\begin{tabular}{|l} 
Seemann Sub SF 2 \\
$v=0,7288 \mathrm{~m} \cdot \mathrm{s}^{-1}$
\end{tabular}}} & \multicolumn{3}{|c|}{ Sporasub Dessault } \\
\hline & & & & & & & $v=0,7$ & $7288 \mathrm{~m}$ & $\mathrm{~s}^{-1}$ \\
\hline Zeit & $\mathrm{VO}_{2}$ & $\mathrm{VCO}_{2}$ & $\mathrm{RQ}$ & $\mathrm{VO}_{2}$ & $\mathrm{VCO}_{2}$ & $\mathrm{RQ}$ & $\mathrm{VO}_{2}$ & $\mathrm{VCO}_{2}$ & $R Q$ \\
\hline$(\min )$ & $(\mathrm{ml} \cdot$ & $\left.\min ^{-1}\right)$ & & $(\mathrm{ml}$ & $\left.\min ^{-1}\right)$ & & $\left(\mathrm{ml}^{\circ}\right.$ & $\left.\min ^{-1}\right)$ & \\
\hline 20,75 & 3190 & 3010 & 0,944 & 570 & 870 & 1,526 & 590 & 770 & 1,305 \\
\hline 21 & 3250 & 3110 & 0,957 & 550 & 840 & 1,527 & 580 & 770 & 1,328 \\
\hline 21,25 & 3020 & 2950 & 0,977 & & & & 0 & 0 & \\
\hline 21,5 & 2800 & 2730 & 0,975 & & & & 10 & 10 & 1 \\
\hline 21,75 & 2470 & 2410 & 0,976 & & & & & & \\
\hline 22 & 2360 & 2390 & 1,013 & & & & & & \\
\hline 22,25 & 2000 & 2270 & 1,135 & & & & & & \\
\hline 22,5 & 1590 & 2000 & 1,258 & & & & & & \\
\hline 22,75 & 1360 & 1860 & 1,368 & & & & & & \\
\hline 23 & 1100 & 1600 & 1,455 & & & & & & \\
\hline 23,25 & 1010 & 1530 & 1,515 & & & & & & \\
\hline 23,5 & 700 & 1110 & 1,586 & & & & & & \\
\hline 23,75 & 490 & 770 & 1,571 & & & & & & \\
\hline 24 & 640 & 960 & 1,5 & & & & & & \\
\hline 24,25 & 20 & 30 & 1,5 & & & & & & \\
\hline
\end{tabular}




\subsubsection{Proband B. Messdaten (Messreihe 4)}

\begin{tabular}{|c|c|c|c|c|c|c|c|c|c|c|c|c|}
\hline \multicolumn{13}{|c|}{ Proband B } \\
\hline & \multirow{2}{*}{\multicolumn{3}{|c|}{\begin{tabular}{|l|l} 
Mares Volo \\
$\mathrm{v}=0,7288 \mathrm{~m} \cdot \mathrm{s}^{-1}$
\end{tabular}}} & \multirow{2}{*}{\multicolumn{3}{|c|}{$\begin{array}{l}\text { Seemann Sub SF } 2 \\
v=0,7288 \mathrm{~m} \cdot \mathrm{s}^{-1}\end{array}$}} & \multirow{2}{*}{\multicolumn{3}{|c|}{$\begin{array}{l}\text { Sporasub Dessault } \\
\mathrm{v}=0,7288 \mathrm{~m} \cdot \mathrm{s}^{-1}\end{array}$}} & \multirow{2}{*}{\multicolumn{3}{|c|}{\begin{tabular}{|l|l|}
$\mid$ Monoflosse \\
$v=0,9,426 \mathrm{~m} \cdot \mathrm{s}^{-1}$
\end{tabular}}} \\
\hline & & & & & & & & & & & & \\
\hline Zeit & $\mathrm{VO}_{2}$ & $\mathrm{VCO}_{2}$ & $R Q$ & $\mathrm{VO}_{2}$ & $\mathrm{VCO}_{2}$ & & $\mathrm{VO}_{2}$ & $\mathrm{VCO}_{2}$ & $R Q$ & $\mathrm{VO}_{2}$ & $\mathrm{VCO}_{2}$ & $R Q$ \\
\hline$(\min )$ & $(\mathrm{ml}$ & $\left.\min ^{-1}\right)$ & & $(\mathrm{ml}$ & $\left.\min ^{-1}\right)$ & & $(\mathrm{ml}$ & $\left.\min ^{-1}\right)$ & & $(\mathrm{ml} \cdot$ & $\left.\min ^{-1}\right)$ & \\
\hline 0,25 & & 750 & 904 & 70 & 60 & 982 & 360 & 330 & & 80 & 650 & 1,121 \\
\hline 0,5 & 480 & 440 & 0 , & & 610 & 0,968 & 10 & 580 & & 0 & 00 & \\
\hline 0,75 & 420 & 410 & 0,976 & 80 & 580 & 1 & 350 & 340 & 0,971 & 50 & 520 & 1,156 \\
\hline 1 & 420 & 420 & 1 & 530 & 540 & 1,019 & 320 & 310 & 0,969 & 400 & 460 & 1,15 \\
\hline 1,25 & 390 & 390 & 1 & 630 & 670 & 1,063 & 400 & 410 & 1,025 & 40 & 280 & 1,167 \\
\hline 1,5 & 400 & 410 & 1,025 & 57 & 650 & 1,14 & 370 & 380 & 1,027 & 00 & 560 & 1,12 \\
\hline 1,75 & 400 & 410 & 1,025 & 460 & 530 & 1,152 & 320 & 340 & 1,063 & 140 & 160 & 1,143 \\
\hline 2 & 470 & 490 & 1,043 & 90 & 600 & 224 & 290 & 310 & & 10 & 400 & \\
\hline 2,25 & 490 & 500 & 1,02 & 450 & 560 & 1,244 & 350 & 380 & 1,086 & 450 & 480 & 1,067 \\
\hline 2,5 & 580 & 600 & 1,034 & 0 & 550 & 1,279 & 50 & 80 & 12 & 40 & 450 & 1,0 \\
\hline 2,75 & 430 & 440 & 1,023 & & 590 & 255 & 280 & 310 & 107 & 30 & 490 & \\
\hline 3 & 490 & 510 & 1,041 & 340 & 430 & 1,265 & 390 & 420 & 1,077 & 460 & 450 & 0,978 \\
\hline 3,25 & 320 & 340 & 1,06 & & 400 & 1,25 & 330 & 350 & 061 & & 490 & \\
\hline 3,5 & 320 & 350 & 1,094 & 210 & 260 & 1,238 & 450 & 490 & 1,089 & 60 & 650 & 0,985 \\
\hline 3,75 & 420 & 470 & 1,119 & 210 & 260 & 1,238 & 330 & 350 & 1,061 & 30 & 720 & 0,986 \\
\hline 4 & & 570 & 1,118 & & 240 & 1,2 & 350 & 380 & 1,086 & 80 & 990 & \\
\hline 4,25 & 570 & 580 & 1,018 & 190 & 240 & 1,263 & 530 & 560 & 1,057 & 1210 & 1140 & 0,942 \\
\hline 4,5 & 1020 & 850 & 0,8 & 16 & 190 & 1,188 & 4 & 4 & 07 & 70 & 370 & \\
\hline 4,75 & 1450 & 1110 & 0,766 & & 190 & 1,188 & 530 & 560 & 057 & 2070 & 1560 & 0,7 \\
\hline 5 & 1520 & 1120 & 0,737 & 150 & 170 & 1,133 & 640 & 650 & 1,016 & 1820 & 1340 & 0,73 \\
\hline 5,25 & 1410 & 1050 & 0,74 & & 150 & 1,071 & 8 & 820 & 0,921 & 2200 & 1620 & 0,736 \\
\hline 5,5 & 1300 & 1000 & 0,769 & 190 & 210 & 1,105 & 890 & 780 & 0,876 & 1860 & 1400 & 0,753 \\
\hline 5,75 & 1450 & 1120 & 0,772 & 330 & 350 & 1,061 & 1050 & 890 & 0,848 & 1830 & 410 & \\
\hline 6 & 1540 & 1190 & 0,773 & 250 & 260 & 1,04 & 1120 & 950 & 0,848 & 2270 & 1770 & 0,78 \\
\hline 6,25 & 1450 & 1140 & 0,786 & 420 & 420 & 1 & 880 & 760 & 0,864 & 1930 & 1520 & 0,788 \\
\hline 6,5 & 1260 & 1010 & 0,802 & 890 & 790 & 0,888 & 930 & 820 & 0,882 & 1540 & 1240 & 0,805 \\
\hline 6,75 & 1330 & 1100 & 0,827 & 1360 & 1060 & 0,779 & 750 & 660 & 0,88 & 1440 & 1150 & 0,799 \\
\hline 7 & 1350 & 1140 & 0,844 & 1600 & 1140 & 0,712 & 940 & 830 & 0,883 & 1780 & 1430 & 0,803 \\
\hline 7,25 & 1270 & 1130 & 0,89 & 2020 & 1410 & 0,698 & 770 & 690 & 0,896 & 890 & 730 & 0,82 \\
\hline 7,5 & 930 & 910 & 0,978 & 1550 & 1100 & 0,71 & 710 & 630 & 0,887 & 1580 & 1340 & 0,848 \\
\hline 7,75 & 660 & 710 & 1,076 & 1750 & 1290 & 0,737 & 670 & 610 & 0,91 & 1380 & 1240 & 0,899 \\
\hline 8 & 990 & 1130 & 1,141 & 1320 & 1000 & 0,758 & 1350 & 1250 & 0,926 & 1210 & 1160 & 0,959 \\
\hline 8,25 & 740 & 850 & 1,149 & 1100 & 850 & 0,773 & 890 & 850 & 0,955 & 1640 & 1590 & 0,97 \\
\hline 8,5 & 940 & 1050 & 1,117 & 1090 & 870 & 0,798 & 1140 & 1100 & 0,965 & 1720 & 1580 & 0,919 \\
\hline 8,75 & 920 & 960 & 1,043 & 1010 & 800 & 0,792 & 1260 & 1190 & 0,944 & 1960 & 1700 & 0,867 \\
\hline 9 & 980 & 940 & 0,959 & 790 & 620 & 0,785 & 1350 & 1230 & 0,911 & 2140 & 1780 & 0,832 \\
\hline 9,25 & 1390 & 1220 & \begin{tabular}{|l|}
0,878 \\
\end{tabular} & 860 & 670 & 0,779 & 1080 & 950 & 0,88 & 2120 & 1740 & 0,821 \\
\hline 9,5 & 1530 & 1270 & 0,83 & 1190 & 950 & 0,798 & 920 & 810 & 0,88 & 2230 & 1840 & 0,825 \\
\hline 9,75 & 1580 & 1290 & 0,816 & 1470 & 1200 & 0,816 & 800 & 690 & 0,863 & 2210 & 1820 & 0,824 \\
\hline 10 & 1690 & 1400 & 0,828 & 820 & 690 & 0,841 & 1320 & 1140 & 0,864 & 2180 & 1830 & 0,839 \\
\hline
\end{tabular}




\begin{tabular}{|c|c|c|c|c|c|c|c|c|c|c|c|c|}
\hline \multicolumn{13}{|c|}{ Proband B } \\
\hline & \multicolumn{2}{|c|}{ Mares Volo } & & \multicolumn{3}{|c|}{ Seemann Sub SF 2} & \multicolumn{3}{|c|}{ Sporasub Dessault } & \multicolumn{2}{|c|}{ Monoflosse } & \\
\hline & \multicolumn{3}{|c|}{$\mathrm{v}=0,7288 \mathrm{~m} \cdot \mathrm{s}^{-1}$} & \multicolumn{3}{|c|}{$\mathrm{v}=0,7288 \mathrm{~m} \cdot \mathrm{s}^{-1}$} & \multicolumn{3}{|c|}{$v=0,7288 \mathrm{~m} \cdot \mathrm{s}^{-1}$} & \multicolumn{3}{|c|}{$v=0,9,426 \mathrm{~m} \cdot \mathrm{s}^{-1}$} \\
\hline Zeit & $\mathrm{VO}_{2}$ & $\mathrm{VCO}_{2}$ & $\mathrm{RQ}$ & $\mathrm{VO}_{2}$ & $\mathrm{VCO}_{2}$ & & $\mathrm{VO}_{2}$ & $\mathrm{VCO}_{2}$ & $R Q$ & $\mathrm{VO}_{2}$ & $\mathrm{VCO}_{2}$ & $R Q$ \\
\hline$(\min )$ & $(\mathrm{ml}$ & $\left.\min ^{-1}\right)$ & & $(\mathrm{ml} \cdot \mathrm{r}$ & $\left.\min ^{-1}\right)$ & & $(\mathrm{ml}$ & $\left.\min ^{-1}\right)$ & & $(\mathrm{ml}$ & $\left.\operatorname{nin}^{-1}\right)$ & \\
\hline 10, & & 40 & & & 140 & & 5 & D0 & & & & \\
\hline 10,5 & 2430 & 2050 & 0,844 & 1140 & 960 & 0 & 1160 & 90 & & & 1460 & \\
\hline 10,75 & 1520 & 1300 & 0,855 & 1270 & 1040 & 0,819 & 1550 & 1310 & 0,845 & 90 & 1390 & \\
\hline 11 & 1590 & 1390 & 0,874 & 1470 & 1190 & & 1720 & 1450 & 0,843 & 1160 & 960 & \\
\hline 11,25 & 1420 & 1250 & 0,88 & 1390 & 1110 & 9 & 1380 & 00 & 0,87 & & 1220 & \\
\hline 11,5 & 1400 & 1240 & 0,886 & 1400 & 1120 & 0,8 & 1040 & 970 & 0,933 & 1290 & 1150 & 0,8 \\
\hline 11,75 & 1050 & 970 & 0,924 & 13 & 1040 & 0,7 & 840 & 30 & 0,988 & 1680 & 1620 & \\
\hline 12 & 1010 & 990 & 98 & & 200 & & 800 & & & & 340 & \\
\hline 12,25 & 490 & 510 & 1,041 & 1520 & 1240 & 0,8 & 760 & 850 & 1,118 & 16 & 1750 & \\
\hline 12,5 & 800 & 850 & 1,063 & 1180 & 980 & & 850 & 30 & & & 1820 & \\
\hline 12,75 & 670 & 730 & 1,09 & 1 & 1240 & & 800 & 90 & & 2270 & 340 & \\
\hline 13 & 910 & 1060 & 1,165 & 1230 & 1010 & 0 & 970 & 960 & 0,99 & 1960 & 1730 & \\
\hline 13,25 & 1180 & 1320 & 1,119 & $10 s$ & 900 & 0 & 1130 & 020 & & 2350 & 2060 & \\
\hline 13,5 & 1570 & 1550 & 0,987 & 1570 & 1340 & 0 & 1430 & 1240 & & 2400 & 2120 & \\
\hline 13,75 & 1730 & 1590 & 0,919 & & 830 & & 1320 & & & & 2130 & \\
\hline 14 & 1920 & 1690 & 0,88 & & 1010 & & & & & & 80 & \\
\hline 14,25 & 2160 & 1860 & 0,861 & 1040 & 1020 & 0,9 & 1400 & 1190 & 0,85 & 1490 & 1330 & 0,8 \\
\hline 14,5 & 1990 & 1700 & 0,854 & 15 & 1430 & & & & & & 320 & \\
\hline 14,75 & 2810 & 2430 & 0,865 & 60 & 860 & 0,8 & 1420 & 1240 & & & 990 & \\
\hline 15 & 430 & 370 & 0,86 & 1430 & 1230 & & 1410 & 1260 & & 15 & 1410 & \\
\hline 15,25 & 50 & 50 & 1 & $15 \mathrm{~s}$ & 1330 & 0,8 & & & & & 450 & \\
\hline 15,5 & 0 & 0 & & 1460 & 1220 & 0,8 & 1250 & 1110 & 0,888 & 1580 & 1620 & \\
\hline 15,75 & 0 & 0 & & 17 & 1450 & & 10 & & & & 60 & \\
\hline 16 & 0 & 0 & & 173 & 1420 & 0 & 1010 & 30 & & 12 & 1530 & \\
\hline 16,25 & 10 & 10 & 1 & 1500 & 1240 & 0,8 & 930 & 900 & & 1200 & 1360 & \\
\hline 16,5 & 0 & 0 & & 17 & 1440 & 0 & & & & & 720 & \\
\hline 16,75 & 0 & 0 & & 1570 & 1300 & 0,828 & 1050 & 1070 & & 2260 & 2070 & \\
\hline 17 & 450 & 390 & 0,867 & 1720 & 1430 & 0,8 & 1010 & 940 & & 26 & 2280 & \\
\hline 17,25 & 860 & 750 & 0,872 & 1 & 980 & 0,8 & 1520 & 340 & & 2560 & 2220 & \\
\hline 17,5 & 1130 & 1010 & 0,894 & 1050 & 900 & 0,8 & 1420 & 1210 & 0,852 & 2290 & 2030 & 0,8 \\
\hline 17,75 & & 770 & 0,906 & & 1300 & & 1750 & & & & 30 & \\
\hline 18 & 800 & 730 & 0,913 & 1030 & 980 & 0,9 & 1630 & 1380 & & 3300 & 2880 & \\
\hline 18,25 & 810 & 730 & 0,901 & & 920 & & 1540 & 1320 & & 3120 & 2750 & \\
\hline 18,5 & 780 & 710 & 0,91 & 1260 & 1300 & 1,032 & 1340 & 1160 & 0,866 & 2180 & 2040 & 0,9 \\
\hline 18,75 & 680 & 630 & 0,926 & 1420 & 1360 & 0,9 & 1680 & 1450 & 0,863 & 2180 & 2080 & 0,9 \\
\hline 19 & 650 & 620 & 0,954 & 1740 & 1540 & 0,885 & 1290 & 1120 & 0,868 & 1660 & 1580 & 0,9 \\
\hline 19,25 & 890 & 880 & 0,989 & 1730 & 1480 & 0,855 & 1240 & 1080 & 0,871 & 1780 & 1720 & 0,96 \\
\hline 19,5 & 950 & 960 & 1,011 & 1770 & 1490 & 0,842 & 1390 & 1220 & 0,878 & 1350 & 1380 & 1,022 \\
\hline 19,75 & 1700 & 1680 & 0,988 & 1900 & 1590 & 0,8 & 1100 & 1000 & 0,909 & 1200 & 1310 & 1,092 \\
\hline 20 & 1610 & 1410 & 0,876 & 2280 & 1940 & 0,851 & 1080 & 1040 & 0,963 & 950 & 1080 & 1,137 \\
\hline 20,25 & 2310 & 1890 & 0,818 & 1650 & 1510 & 0,863 & 850 & 860 & 1,012 & 990 & 1180 & 1,192 \\
\hline 20,5 & 2290 & 1840 & 0,803 & 1860 & 1630 & 0,87 & 850 & 910 & 1,071 & 750 & 920 & 1,22 \\
\hline
\end{tabular}




\begin{tabular}{|c|c|c|c|c|c|c|c|c|c|c|c|c|}
\hline \multicolumn{13}{|c|}{ Proband B } \\
\hline & \multirow{2}{*}{\multicolumn{3}{|c|}{$\begin{array}{l}\text { Mares Volo } \\
\mathrm{v}=0,7288 \mathrm{~m} \cdot \mathrm{s}^{-1}\end{array}$}} & \multirow{2}{*}{\multicolumn{3}{|c|}{$\begin{array}{l}\text { Seemann Sub SF } 2 \\
v=0,7288 \mathrm{~m} \cdot \mathrm{s}^{-1}\end{array}$}} & \multirow{2}{*}{\multicolumn{3}{|c|}{\begin{tabular}{|l} 
Sporasub Dessault \\
$v=0,7288 \mathrm{~m} \cdot \mathrm{s}^{-1}$
\end{tabular}}} & \multicolumn{2}{|c|}{ Monoflosse } & \\
\hline & & & & & & & & & & $v=0,9$ & $9,426 \mathrm{~m}$ & $\cdot s^{-1}$ \\
\hline Zeit & $\mathrm{VO}_{2}$ & $\mathrm{VCO}_{2}$ & $R Q$ & $\mathrm{VO}_{2}$ & $\mathrm{VCO}_{2}$ & & $\mathrm{VO}_{2}$ & $\mathrm{VCO}_{2}$ & $R Q$ & $\mathrm{VO}_{2}$ & $\mathrm{VCO}_{2}$ & $R Q$ \\
\hline$(\min )$ & $(\mathrm{ml} \cdot \mathrm{r}$ & $\left.\min ^{-1}\right)$ & & $(\mathrm{ml} \cdot \mathrm{r}$ & $\left.\min ^{-1}\right)$ & & $(\mathrm{ml}$ & $\left.\min ^{-1}\right)$ & & $(\mathrm{ml} \cdot \mathrm{r}$ & $\left.\min ^{-1}\right)$ & \\
\hline 20,75 & 2480 & 2000 & 0,806 & 1620 & 1440 & 0,889 & 500 & 560 & 1,12 & 520 & 670 & 1,288 \\
\hline 21 & 2320 & 1950 & 0,841 & 1530 & 1340 & 0,876 & 740 & 860 & 1,162 & 210 & 280 & 1,333 \\
\hline 21,25 & 2310 & 1990 & 0,861 & 1450 & 1270 & 0,876 & 640 & 740 & 1,156 & 280 & 370 & 1,321 \\
\hline 21,5 & 2280 & 1990 & 0,873 & 1290 & 1150 & 0,891 & 580 & 690 & 1,19 & 450 & 610 & 1,356 \\
\hline 21,75 & 1820 & 1610 & 0,885 & 1100 & 1020 & 0,927 & 540 & 650 & 1,204 & 550 & 720 & 1,309 \\
\hline 22 & 1710 & 1490 & 0,871 & 750 & 720 & 0,96 & & & & & & \\
\hline 22,25 & 1860 & 1610 & 0,866 & 970 & 970 & 1 & & & & & & \\
\hline 22,5 & 1600 & 1410 & 0,881 & 670 & 700 & 1,045 & & & & & & \\
\hline 22,75 & 1340 & 1220 & 0,91 & 790 & 880 & 1,114 & & & & & & \\
\hline 23 & 1140 & 1120 & 0,982 & 460 & 540 & 1,174 & & & & & & \\
\hline 23,25 & 1260 & 1320 & 1,048 & 240 & 300 & 1,25 & & & & & & \\
\hline 23,5 & 1440 & 1570 & 1,09 & 320 & 390 & 1,219 & & & & & & \\
\hline 23,75 & 1120 & 1160 & 1,036 & 380 & 490 & 1,289 & & & & & & \\
\hline 24 & 1650 & 1600 & 0,97 & 380 & 500 & 1,316 & & & & & & \\
\hline 24,25 & 1570 & 1420 & 0,904 & & & & & & & & & \\
\hline 24,5 & 2380 & 2050 & 0,861 & & & & & & & & & \\
\hline 24,75 & 2360 & 2040 & 0,864 & & & & & & & & & \\
\hline 25 & 2450 & 2180 & 0,89 & & & & & & & & & \\
\hline 25,25 & 2370 & 2130 & 0,899 & & & & & & & & & \\
\hline 25,5 & 2100 & 1930 & 0,919 & & & & & & & & & \\
\hline 25,75 & 2320 & 2110 & 0,909 & & & & & & & & & \\
\hline 26 & 2160 & 1960 & 0,907 & & & & & & & & & \\
\hline 26,25 & 2640 & 2430 & 0,92 & & & & & & & & & \\
\hline 26,5 & 2440 & 2270 & 0,93 & & & & & & & & & \\
\hline 26,75 & 2150 & 2030 & 0,944 & & & & & & & & & \\
\hline 27 & 1930 & 1890 & 0,979 & & & & & & & & & \\
\hline 27,25 & 1310 & 1370 & 1,046 & & & & & & & & & \\
\hline 27,5 & 1040 & 1160 & 1,115 & & & & & & & & & \\
\hline 27,75 & 880 & 1030 & 1,17 & & & & & & & & & \\
\hline 28 & 480 & 580 & 1,208 & & & & & & & & & \\
\hline 28,25 & 680 & 840 & 1,235 & & & & & & & & & \\
\hline 28,5 & 490 & 610 & 1,245 & & & & & & & & & \\
\hline 28,75 & 560 & 700 & 1,25 & & & & & & & & & \\
\hline 29 & 470 & 610 & 1,298 & & & & & & & & & \\
\hline 29,25 & 530 & 680 & 1,283 & & & & & & & & & \\
\hline 29,5 & 320 & 410 & 1,281 & & & & & & & & & \\
\hline
\end{tabular}


8.1.14 Gegenüberstellung aller Messwerte mit den nach dem Einschwingverhalten relevanten Messwerten (Messreihe 4)
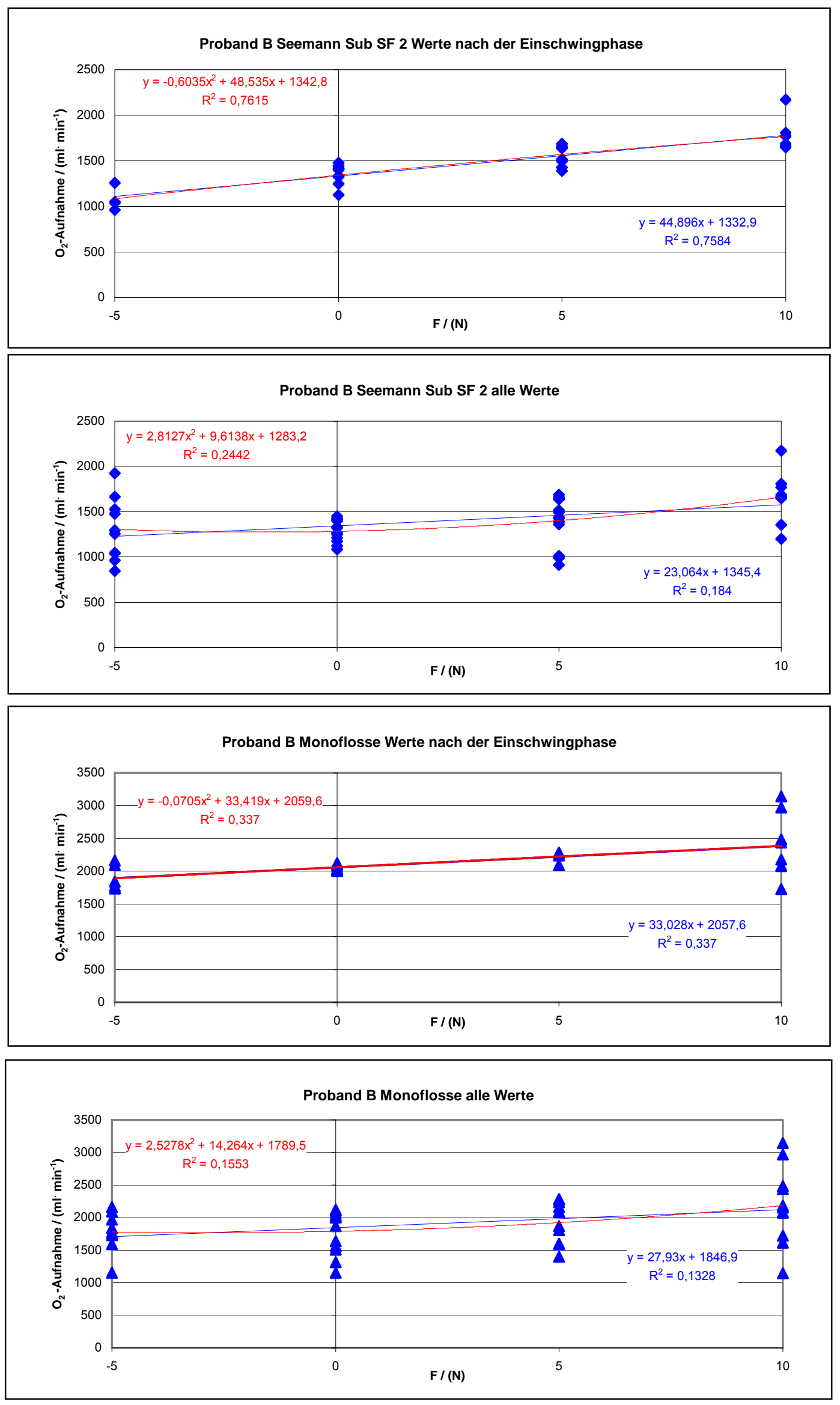

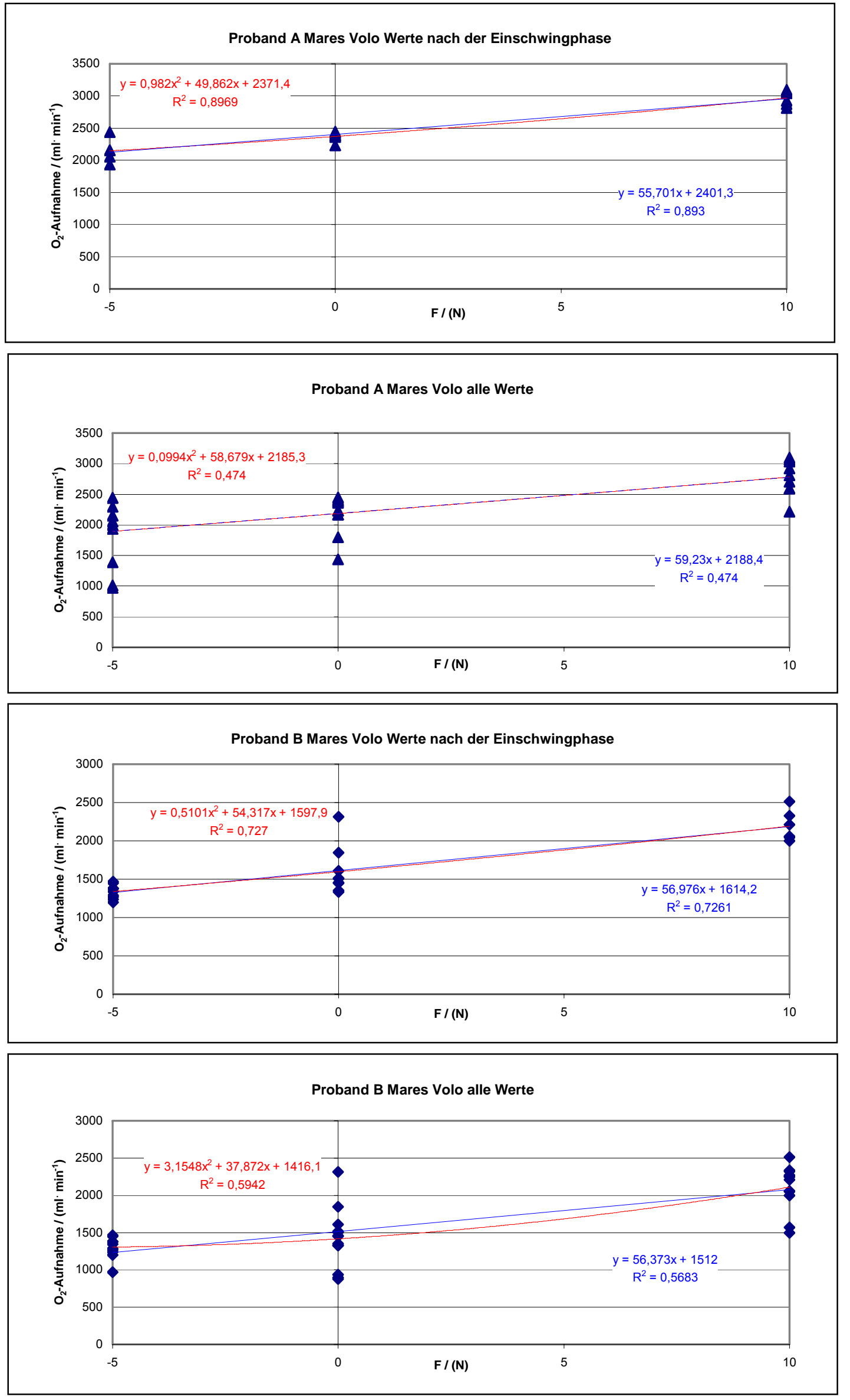

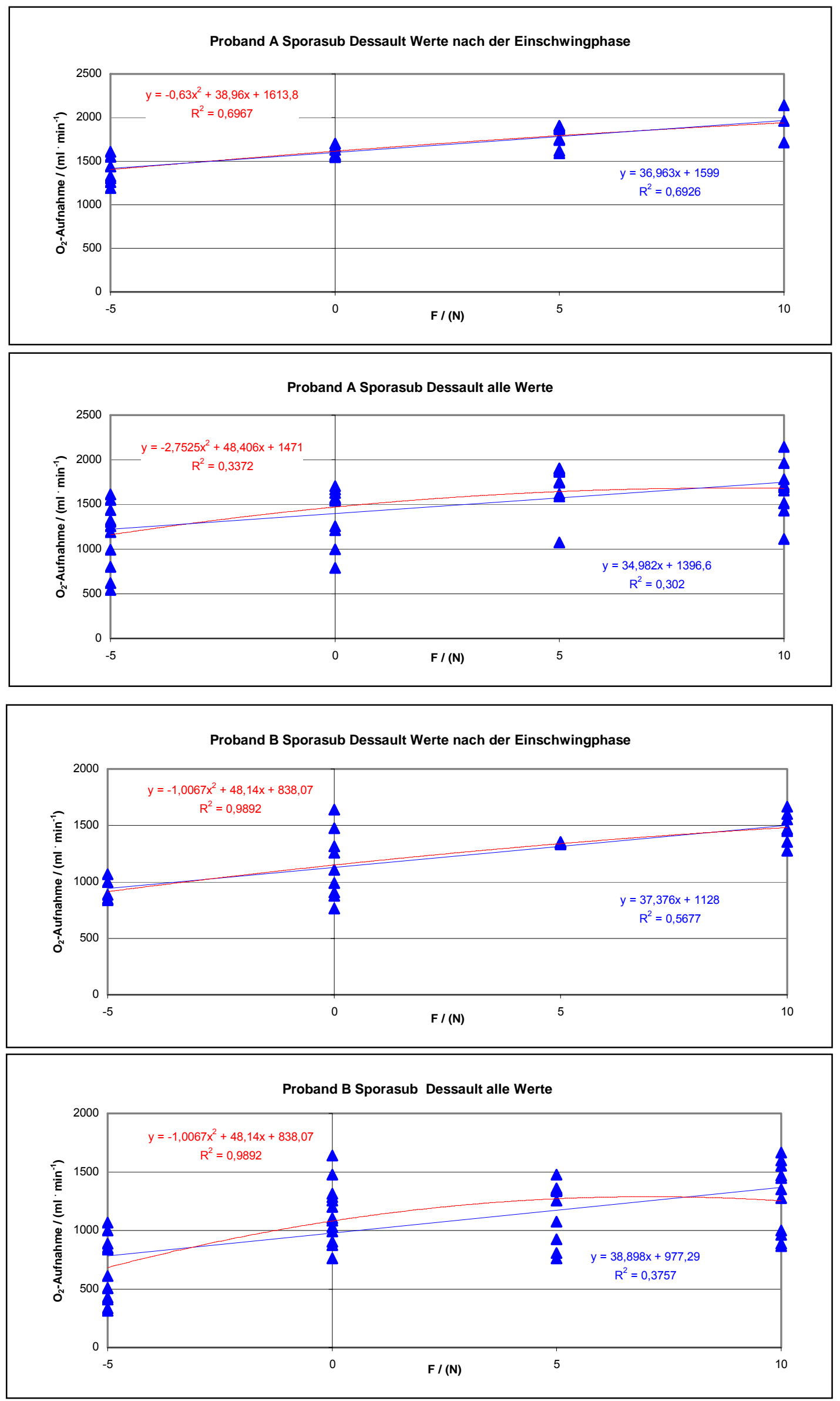


\subsection{Bilder}

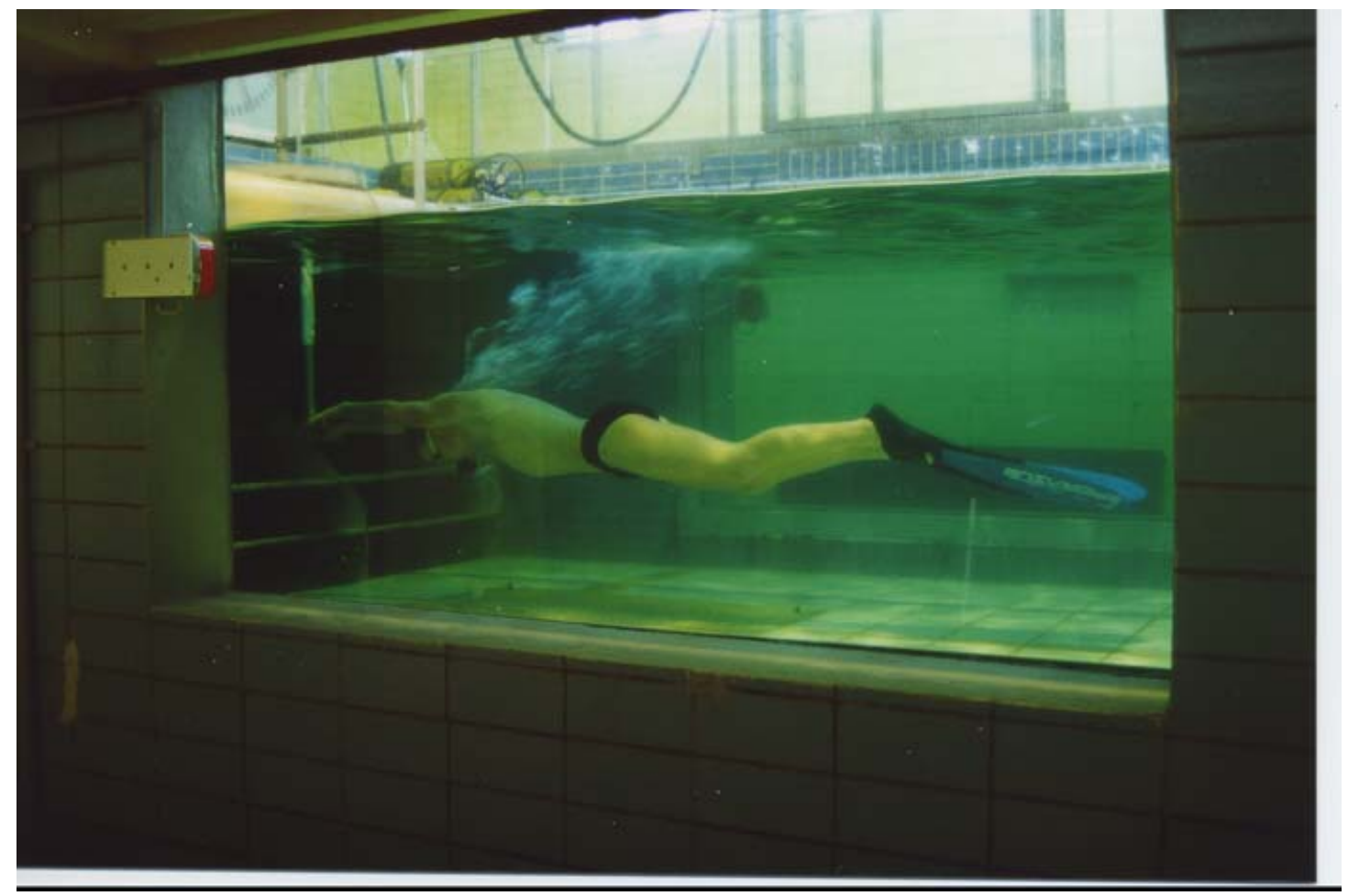

Bild 1: Passives Schleppen im Strömungskanal. Proband B Sporasub Dessault. Seitenansicht durch das Sichtfenster des Strömungskanals. Der Proband hält sich am Kraftaufnehmer fest.

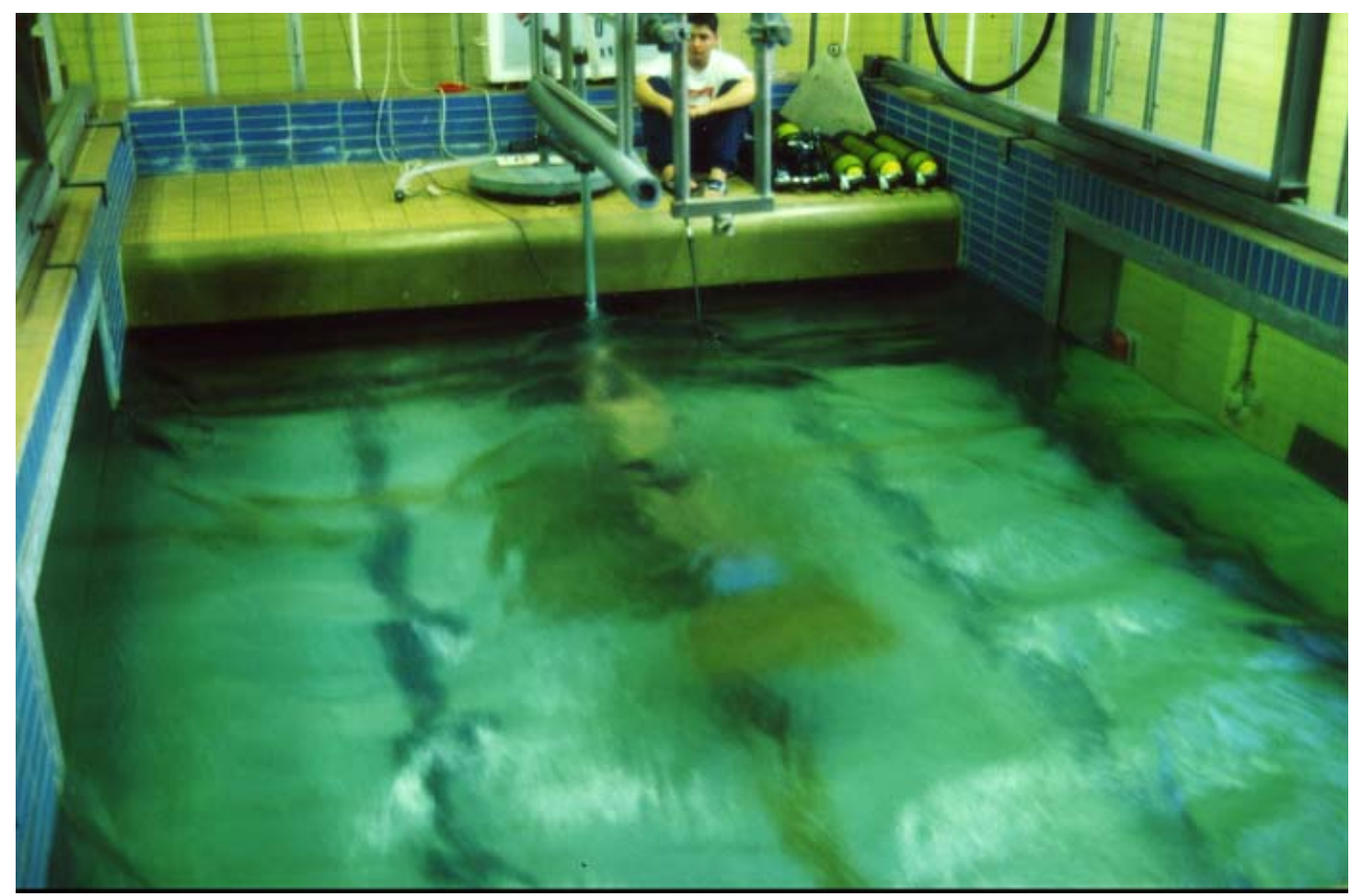

Bild 2: Passives Schleppen im Strömungskanal. Proband B Monoflosse. Sicht gegen die Strömung. Der Proband hält sich am Kraftaufnehmer fest. Rechts neben der Sicherungsperson liegen die Druckluftflaschen mit dem Luftvorrat. 


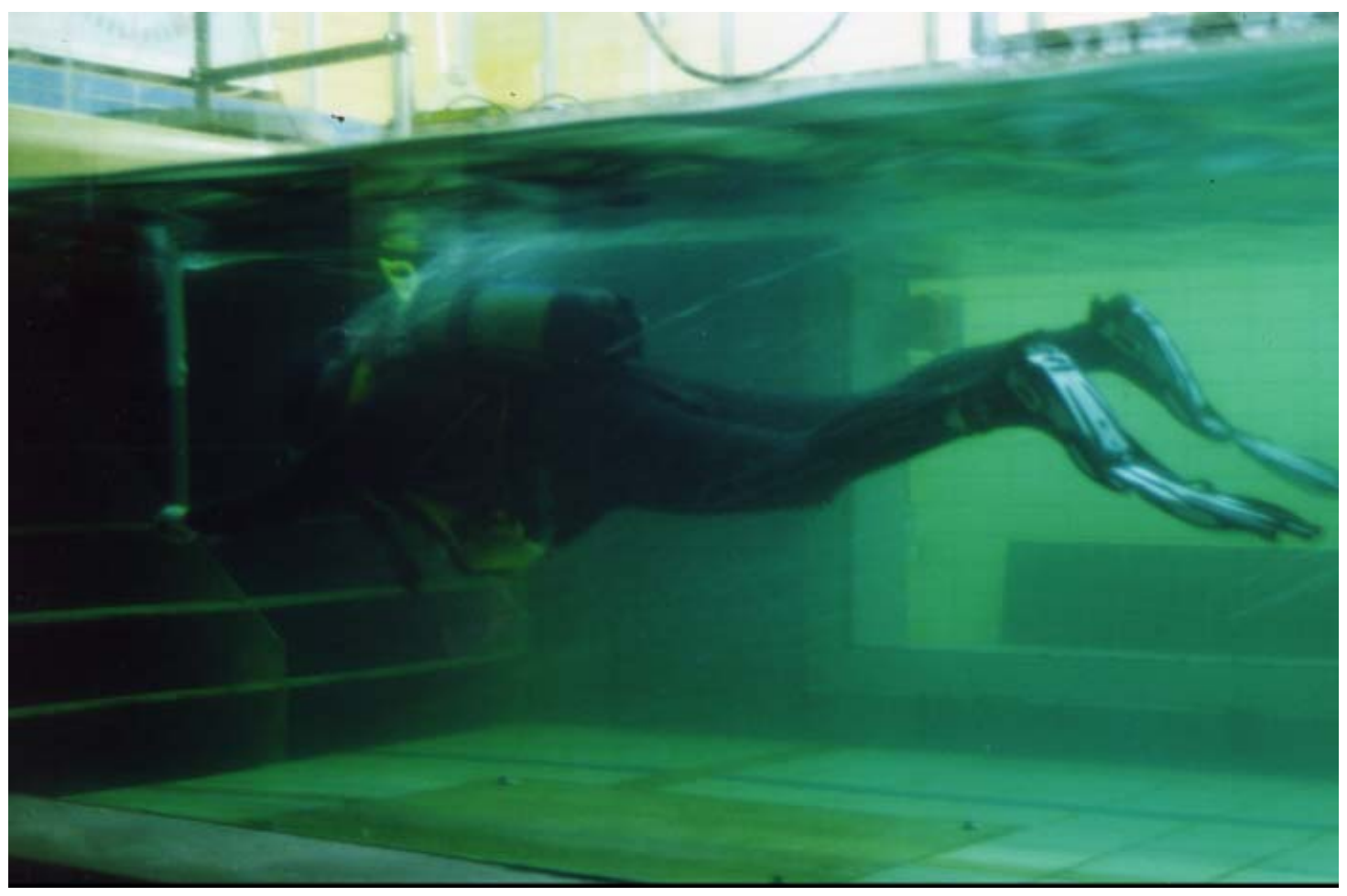

Bild 3: Passives Schleppen im Strömungskanal. Der Proband mit kompletter Tauchausrüstung und Ausrüstungsgegenstand Kragenweste. Seitenansicht durch das Sichtfenster des Strömungskanals.

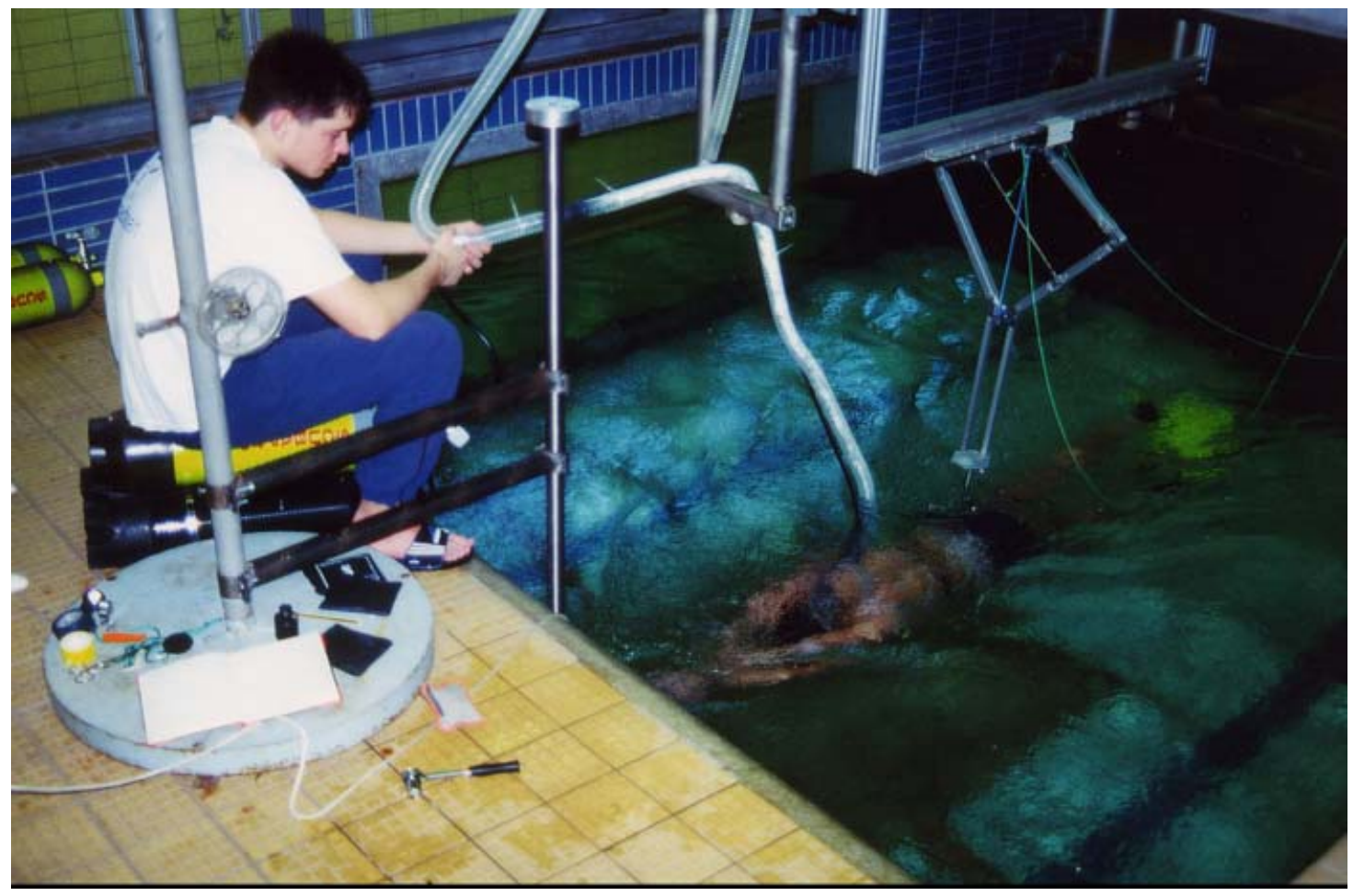

Bild 4: Gasanalytik. Proband B mit Seemann Sub SF 2. Sicht aus Strömungsrichtung. Links befindet sich die Sicherungsperson, welche die Schläuche zur Luftzuführung und Gasableitung kontrolliert. Der Proband ist in der in allen Ebenen frei beweglichen Aufhängung eingehängt. 


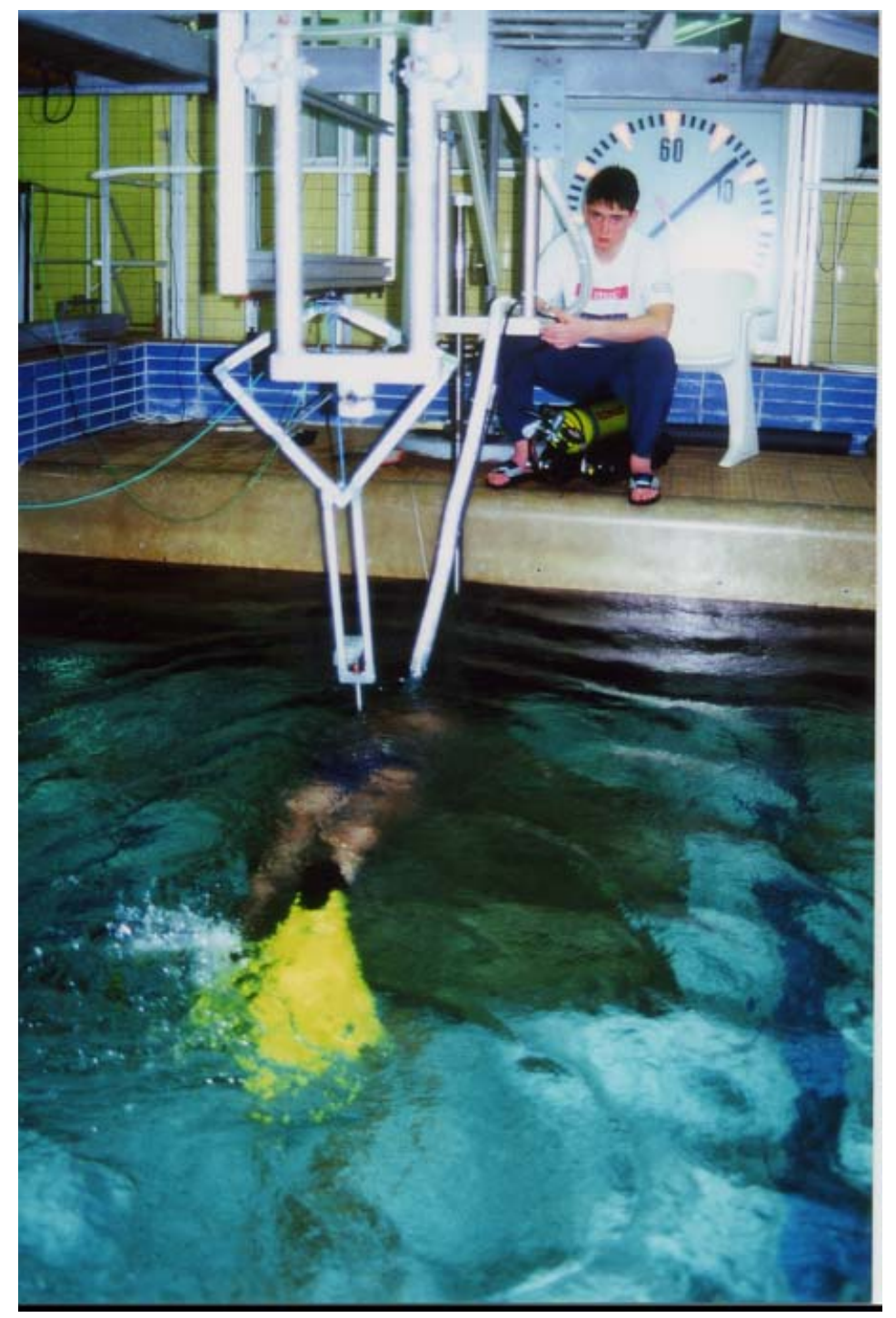

Bild 5: Gasanalytik. Proband B mit Seemann Sub SF 2. Sicht gegen die Strömungsrichtung.

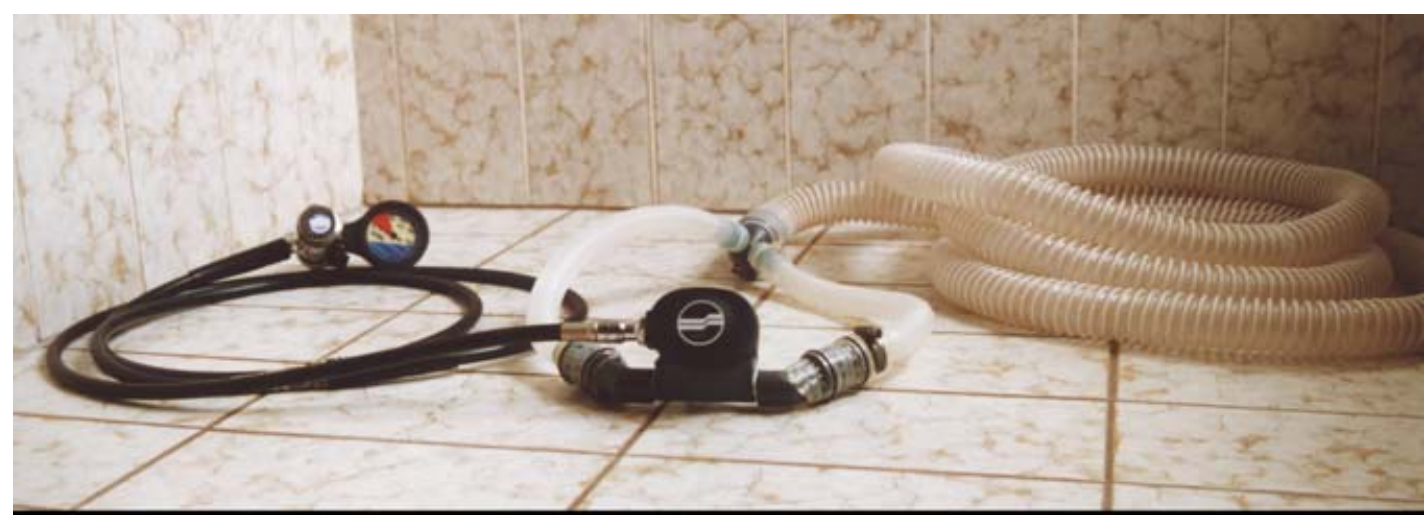

Bild 6: Der Atemregler Sherwood New Brut mit umgebungsdruckgesteuertem Ausatemventil. Links ist die 1. Stufe mit Manometer und Mitteldruckschlauch zur 2. Stufe. Die umgebungsdruckgesteuerten Ausatemventile sind mit Winkelstücken an der 2. Stufe angebracht. Die daran befestigten Schläuche werden (hinter dem Kopf des Probanden) zusammengeführt und die Ausatemluft über den Schlauch (rechts) zum Analysegerät abgeleitet. 


\subsection{CD-ROM}

Auf der beiliegenden CD-ROM sind die Messdaten abgelegt, die auf Grund der Datenmenge den Rahmen einer Druckversion sprengen würden.

Filmdokumente der Versuche sind ebenfalls zu finden. Die Filmaufnahmen wurden durch das Seitenfenster des Strömungskanals aufgenommen. In den Filmtiteln wird Sporasub für Sporasub Dessault, Volo für Mares Volo, Seemann Sub für Seemann Sub SF 2 und Wing für Wing Jacket gesetzt.

Die Geschwindigkeit ist in $(\mathrm{m} / \mathrm{s})$ statt $\left(\mathrm{m} \cdot \mathrm{s}^{-1}\right)$ geschrieben und in den Abstufungen 0,3 $\mathrm{m} / \mathrm{s}, 0,6 \mathrm{~m} / \mathrm{s}, 0,9 \mathrm{~m} / \mathrm{s}, 1,2 \mathrm{~m} / \mathrm{s}$ und $1,5 \mathrm{~m} / \mathrm{s}$ angegeben. Diese stehen für die realen Geschwindigkeiten, die sich aus Messreihe 1 ergeben.

Systemvoraussetzungen Windows 2000 oder höher, Microsoft Excel 2000 oder höher, Microsoft Media Player 7.0 oder höher. Die Dateien werden mit Doppelklick geöffnet. Folgende Dateien sind auf der CD-ROM:

\section{Dateiname: Bedingungen_der_Strömung_im_Kanal.xls}

In der Datei sind Tabellenblätter zu finden. Die Kennzeichnung ist z.B. wie folgt: v03h080.

v steht für Geschwindigkeit.

03 steht für die eingestellte Geschwindigkeit (oder 06, 08, 09, 1,2 1,5). $\mathrm{h}$ steht für hinten (alternativ $\mathrm{m}$ für Mitte und $\mathrm{v}$ für vorne). 080 steht für die Tiefe von $80 \mathrm{~mm}$ (alternativ 470 für $470 \mathrm{~mm}$ ) Jedes Tabellenblatt enthält sieben Spalten. In der ersten Spalte ist die Zeit, in der zweiten Spalte die am Kanal eingestellte Geschwindigkeit eingetragen. Spalte drei bis sieben enthalten die Messdaten von Messflügel eins bis fünf. Wegen der besseren Lesbarkeit erfolgt ein Zeilenumbruch nach 1,09 min und 2,19 $\min$.

\section{Dateiname: Kalibrierung_des_Kraftaufnehmers.xls}

Diese Datei enthält ein Tabellenblatt mit zwei Spalten. Spalte eins enthält die Zeit in min zwischen 0 und 9,99 min. Spalte zwei stellt die gemessenen Kraftwerte in $\mathrm{N}$ dar.

\section{Dateiname: Kraftwerte_Auftriebskörper_Proband_A.xls}

Die Datei enthält vier Tabellenblätter. Jedes Tabellenblatt steht für die komplette Versuchsreihe mit einem anderen Auftriebskörper. Der Name jedes Blattes nennt den jeweiligen Auftriebskörper. In der ersten Zeile jedes Blattes sind die genauen Ausrüstungsdetails zu finden. In Zeile drei sind die Strömungsgeschwindigkeiten eingetragen. Spalte eins zeigt den Messzeitraum von $0 \mathrm{sec}-2,99983333 \mathrm{sec}$. In Spalte zwei bis sechs sind die jeweiligen Messwerte in $\mathrm{N}$ abgebildet.

\section{Dateiname: Kraftwerte_Auftriebskörper_Proband_B.xls}

Siehe Beschreibung „Kraftwerte_Auftriebskörper_Proband_A.xls“ analog für Proband B. 


\section{Dateiname: Kraftwerte_Flossen_Proband_A.xls}

Die Datei enthält vier Tabellenblätter. Jedes Tabellenblatt steht für die komplette Versuchsreihe mit einer anderen Flosse. Der Name jedes Blattes nennt die jeweilige Flosse. In der ersten Zeile jedes Blattes sind die Details genannt. In Zeile drei sind die Strömungsgeschwindigkeiten eingetragen. Spalte eins zeigt den Messzeitraum von 0 sec - 2,99983333 sec. In Spalte zwei bis sechs sind die jeweiligen Messwerte in $\mathrm{N}$ abgebildet.

\section{Dateiname: Kraftwerte_Flossen_Proband_B.xls}

Siehe Beschreibung „Kraftwerte_Flossen_Proband_A.xls“ analog für Proband B.

\section{Dateiname: Strömungsverhältnisse_im_Kanal.mpeg}

Der Film zeigt in einem Zusammenschnitt die Durchführung der Strömungsmessung. Die Titel erklären die jeweilige Sequenz. Dauer: 2:50 $\min$.

\section{Dateiname: Schleppversuche_mit_untersch_Ausrüstungsgeg.mpeg}

In diesem Film sind die Versuche Proband A HUB, B Kragenweste, A Wing Jacket, B Wing Jacket und A Kragenweste in Ausschnitten dokumentiert. Auch hier erklären die Titel die jeweilige Filmsequenz. Dauer: 7:43 min.

\section{Dateiname: Schleppversuche_mit_unterschiedlichen_Flossen.mpeg}

In dem Film sind die Versuche Proband A Seemann Sub SF 2 beginnend mit $0,9 \mathrm{~m} \cdot \mathrm{s}^{-1}$, B Sporasub Dessault, A Sporasub Dessault und B Mares Volo in Ausschnitten dokumentiert. Auch hier erklären die Titel die jeweilige Filmsequenz. Dauer: 6:25 min.

\section{Dateiname: Gasanalytik.mpeg}

Dieser Film zeigt die Versuche Proband B mit Mares Volo und Proband A mit Mares Volo als Zusammenschnitt. Der Abbruch bei Proband B ist zwischen 1:40 min und 2:05 min zu sehen. Die Filmtitel erklären den jeweiligen Ausschnitt. Dauer: 4:14 min. 
Ich versichere, dass ich die eingereichte Dissertation

„Die Belastung und Belastbarkeit von Schwimmtauchern unter besonderer Berücksichtigung der Konfiguration der Ausrüstung sowie des passiven und dynamischen Widerstands dargestellt - anhand von objektivierenden Untersuchungsmethoden“"

selbstständig und ohne unerlaubte Hilfsmittel verfasst habe. Anderer als der von mir angegebenen Hilfsmittel und Schriften habe ich mich nicht bedient. Alle wörtlich oder sinngemäß den Schriften anderer Autoren entnommenen Stellen habe ich kenntlich gemacht.

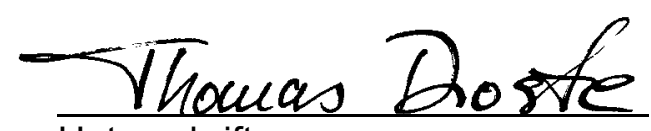

Unterschrift 
Herrn Prof. Dr. Dr. André Niklas danke ich für die Betreuung der Arbeit und Diskussionsbereitschaft. Für die Übernahme des Zweitgutachtens danke ich Herrn Prof. Dr. Arndt Krüger. Für die Übernahme des Drittgutachtens danke ich Herrn Prof. Dr. Gernot Badtke.

Für die Unterstützung und die kritische Durchsicht der Manuskripte gilt mein Dank Dr. Michael Almeling, insbesondere auch für das offene Ohr bei allen Fragen und Problemen.

Herrn Ralf Hottowitz danke ich für die technische Unterstützung und Hilfe bei der Durchführung der Untersuchungen.

Frau Gaby Henken danke ich für die Korrekturen und Herrn Hartmut Kuhfahl für die Einweisung in die Filmbearbeitung.

Mein besonderer Dank gilt meiner Ehefrau für ihre Unterstützung und ihre Geduld bei dem langjährigen Vorhaben. 


\section{Lebenslauf}

Thomas Droste, geb. Büscher

\section{Persönliche Daten}

Geburtsdatum und Ort Familienstand

Staatsangehörigkeit

\section{Schulabschluss}

1975-06

\section{Zivildienst}

1976-04 - 1977-07

\section{Studium}

1977-09 - 1987-03

$1984-11-1986-04$

\section{Berufstätigkeit}

1986-07 - 2001-01

1990-02 - 1991-03

2001-02 - 2003-07

seit 2003-08

Weitere Schwerpunkte

$$
\begin{aligned}
& \text { 1986-08 - heute } \\
& \text { 1986-07 - 1992-06 } \\
& \text { 1993-10 - 1996-06 }
\end{aligned}
$$

seit 1997-10
24.September 1956 in Essen/Ruhr verheiratet, 2 Kinder deutsch

Abitur in Essen/Ruhr.

Zivildienst.

Georg-August-Universität, Studium der Sozialwissenschaften, erweitert zu Sozialkunde und Sportwissenschaft für das Lehramt an Realschulen. Studienschwerpunkt: Didaktik und Methodik, Wassersport. Abschluss mit dem 1. Staatsexamen für das Lehramt an Realschulen.

Ausbildungsseminar Göttingen und Kooperative Gesamtschule Moringen. Vorbereitungsdienst für das Lehramt an Realschulen mit Abschluss des 2. Staatsexamens.

selbstständige Tätigkeit im Unternehmen „tommys tauchladen“" in Göttingen.

Geschäftsführung der Flugbörse Göttingen D\&O Reisen $\mathrm{GmbH}$.

Realschullehrer in Zetel

Realschullehrer in Hattorf.

Ausbildungstätigkeit im Tauchsport u.a. als stellvertretender Landesausbildungsleiter für Niedersachsen.

Fachreferent im Tauchsportlandesverband Niedersachsen für Schulsport.

Vertreter des Institutes für Sport- und Sportwissenschaft der Universität Göttingen in der Fachkonferenz des Verbandes Deutscher Sporttaucher e.V..

Lehrauftrag „Sporttauchen“ am Institut für Sport und Sportwissenschaft im Fachbereich Sozialwissenschaften der Universität Göttingen. 